\title{
Hydrologic Resources Management Program and Underground Test Area Project FY2005 Progress Report
}

\author{
EDITORS \\ Mavrik Zavarin \\ Annie B. Kersting \\ Rachel E. Lindvall \\ Timothy P. Rose
}

\section{AUTHORS}

Gail F. Eaton, Victoria Genetti, Qinhong (Max) Hu, G. Bryant Hudson, Annie B. Kersting, Rachel

E. Lindvall, Jean E. Moran, Gregory J. Nimz, Erick C. Ramon, Timothy P. Rose, Lindsay Shuller,

Ross W. Williams, Mavrik Zavarin, Pihong Zhao

March 2007

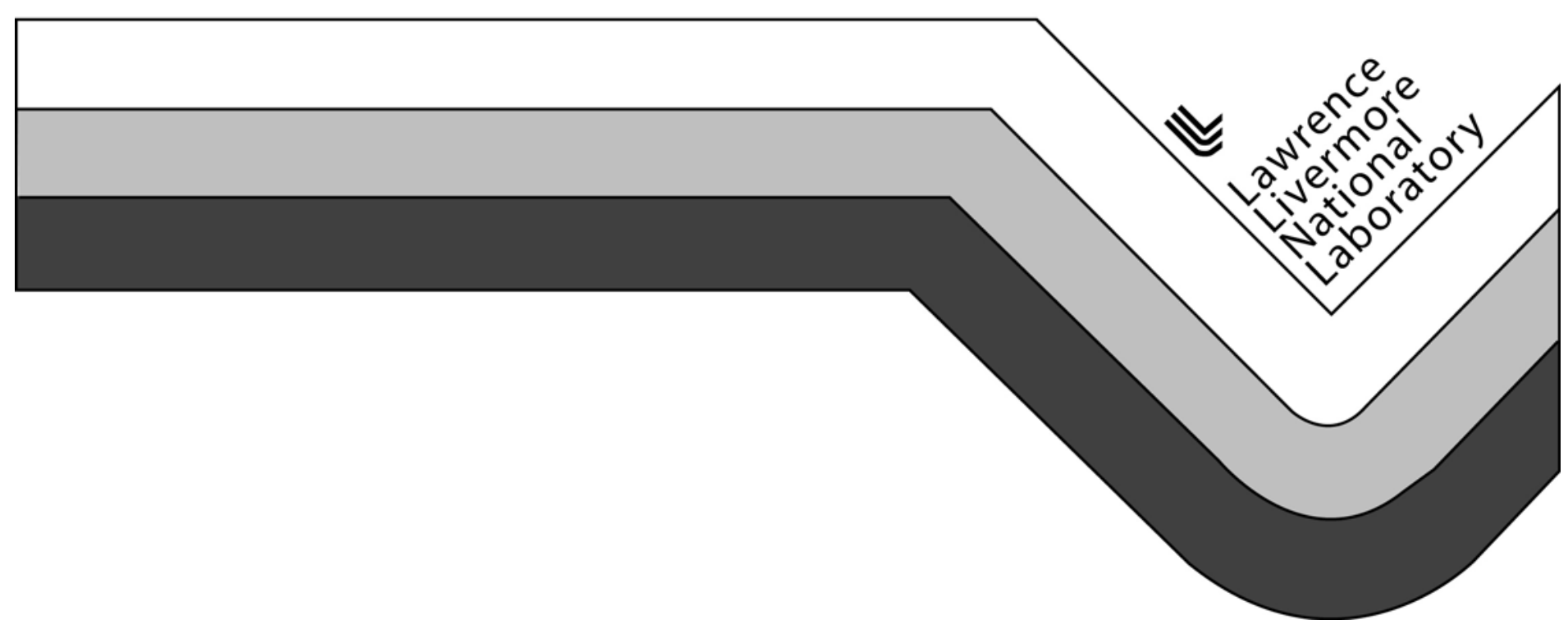




\section{DISCLAIMER}

This document was prepared as an account of work sponsored by an agency of the United States Government. Neither the United States Government not the University of California nor any of their employees, makes any warranty, express or implied, or assumes any legal liability or responsibility for the accuracy, completeness, or usefulness of any information, apparatus, product, or process disclosed, or represents that its use would not infringe privately owned right. Reference herein to any specific commercial product, process or service by trade name, trademark, manufacturer, or otherwise, does not necessarily constitute or imply its endorsement, recommendation, or favoring by the United States Government or the University of California. The views and opinions of authors expressed herein do not necessarily state or reflect those of the United States Government or the University of California, and shall not be used for advertising or product endorsement purposes.

This work was funded by the Hydrologic Resources Management Program and the Underground Test Area Project of the National Nuclear Security Administration, U.S. Department of Energy, Nevada Site Office. Work performed under the auspices of the U. S. Department of Energy by the University of California Lawrence Livermore National Laboratory under Contract W-7405-Eng-48.

This report has been reproduced directly from the best available copy.

Available for a processing fee to U.S. Department of Energy and its contractors, in paper, from:

U.S. Department of Energy

Office of Scientific and Technical Information

P.O. Box 62, Oak Ridge, TN 37831-0062

Phone: 865.576 .8401

Fax: 865.576.5728

Email: reports@adonis.osti.gov

http://www.doe.gov.bridge

Available for sale to the public from:

U.S. Department of Commerce

National Technical Information Service

5285 Port Royal Road

Springfield, VA 22161

Phone: 800.553 .6847

Fax: 703.605.6900

Email: orders@,ntis.fedworld.gov

Online ordering: http://www.ntis.gov/ordering.htm

OR

Lawrence Livermore National Laboratory Technical Information Department's Digital Library

http://www.llnl.gov/tid/Library.html 


\title{
Hydrologic Resources Management Program and Underground Test Area Project FY 2005 Progress Report
}

\author{
EDITORS \\ Mavrik Zavarin \\ Annie B. Kersting \\ Rachel E. Lindvall \\ Timothy P. Rose \\ AUTHORS \\ Gail F. Eaton \\ Victoria Genetti \\ Qinhong (Max) Hu \\ G. Bryant Hudson \\ Annie B. Kersting \\ Rachel E. Lindvall \\ Jean E. Moran \\ Gregory J. Nimz \\ Erick C. Ramon \\ Timothy P. Rose \\ Lindsay Shuller \\ Ross W. Williams \\ Mavrik Zavarin \\ Pihong Zhao
}

March 2007 



\section{Table of Contents}

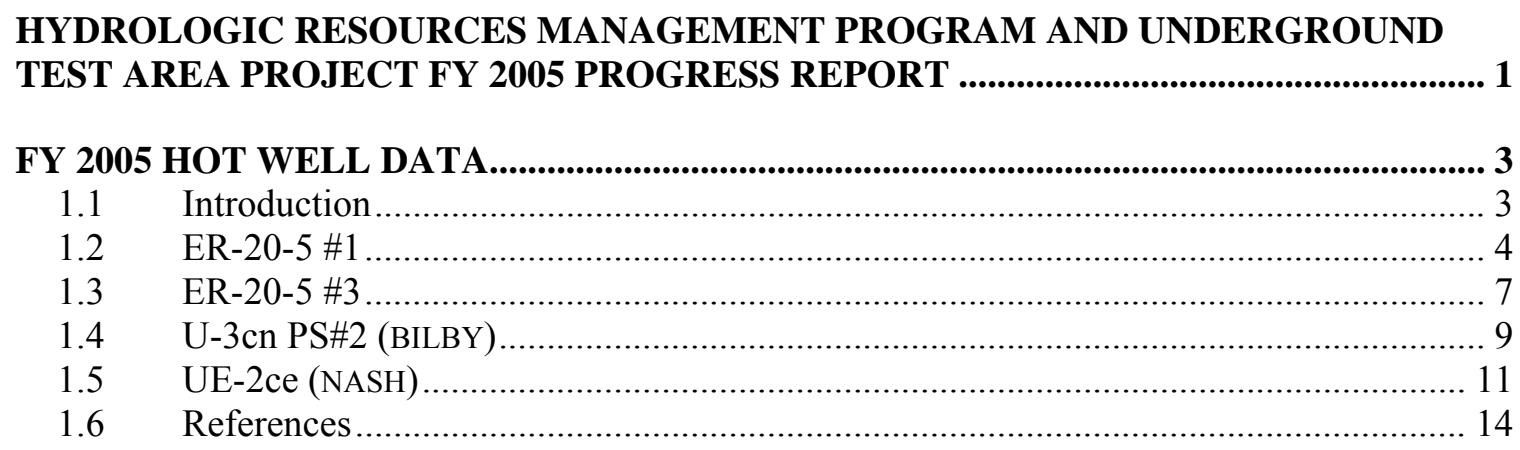

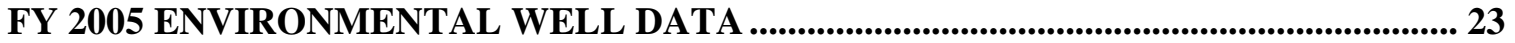

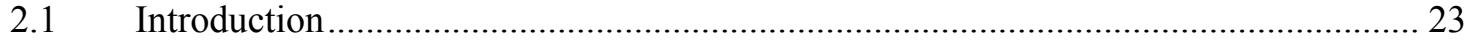

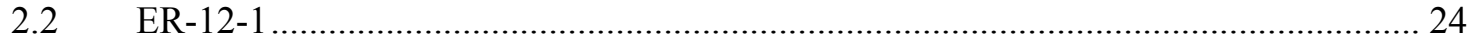

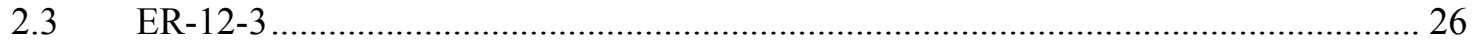

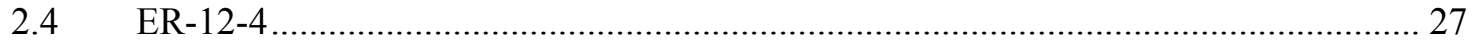

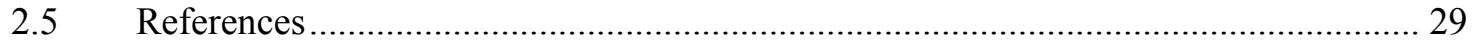

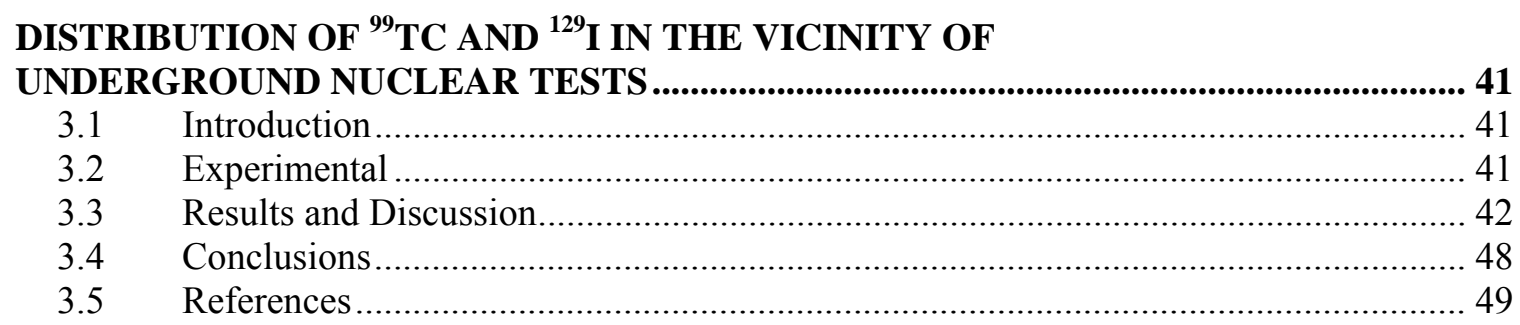

\begin{tabular}{|c|c|}
\hline MELT & LASS \\
\hline 4.1 & 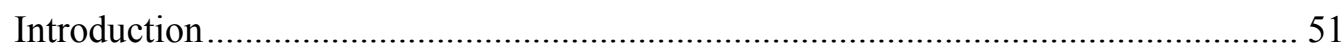 \\
\hline 4.2 & 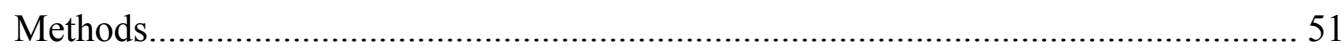 \\
\hline 4.3 & Procedure .. \\
\hline 4.4 & Results and Discussion..................... \\
\hline 4.5 & Summary \\
\hline 4.6 & References \\
\hline 4.7 & 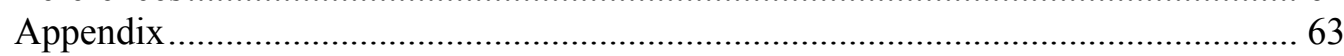 \\
\hline
\end{tabular}

STABLE ISOTOPE INVESTIGATION OF PRECIPITATION AND RECHARGE

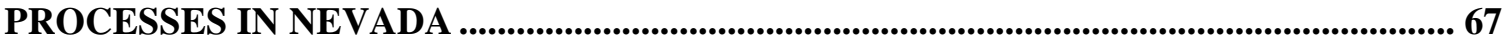

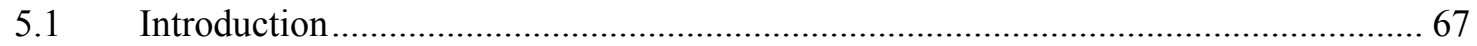

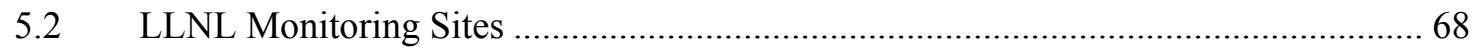

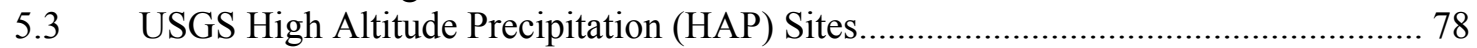

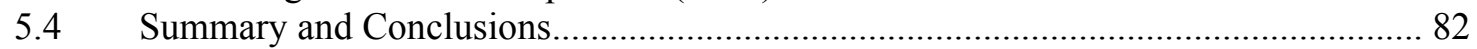

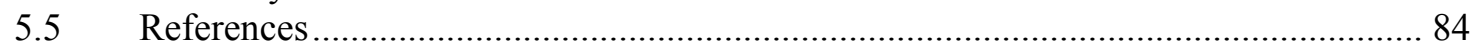

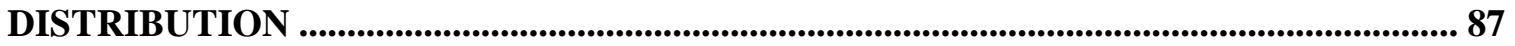




\section{List of Figures}

Figure 1.1 Map of the NTS showing Hotwell sampling locations for FY2005 .......................... 4

Figure 1.2 Location of the ER-20-5 well cluster relative to the TYBO and BENHAM tests.

From Kersting et al. (1999). Cavity size is not to scale................................................. 5

Figure 1.3 The BILBY near-field. From Buddemeier and Isherwood (1985)............................... 9

Figure 1.4 The NASH near-field geometry and water level measurements as a function

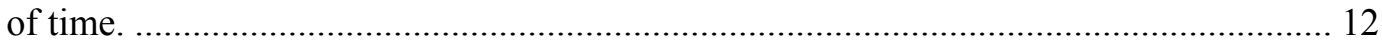

Figure 2.1 Map of the NTS showing environmental well sampling locations for FY2005 ....... 24

Figure 3.1 Cross section of the CHANCELLOR cavity, chimney, and post-shot boreholes showing the locations of sidewall core samples that were used in this investigation. Main hole (PS1A); first sidetrack (PS1AS) and second sidetrack (PS1ASS). MD:

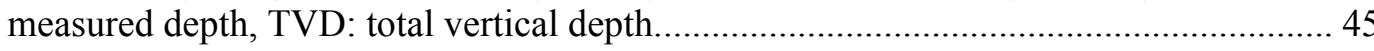

Figure 4.1 Centrifuge tubes containing primary and secondary minerals................................. 53

Figure 4.2 Eh-pH values for the $120^{\circ} \mathrm{C}$ (blue) and $200^{\circ} \mathrm{C}$ (red) samples superimposed on the Eh-pH diagram and data from Zavarin et al. (2004). .......................................... 54

Figure 4.3 SEM Image of (A) mordenite morphology, (B) clay-like morphology, and

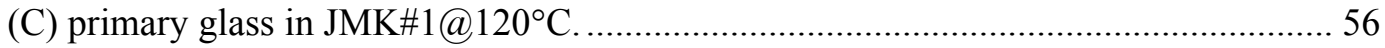

Figure 4.4 SEM image of (A) clay structure and (B) 2 other morphologies observed in JMK\#3@120ㄷ….............................................................................................. 56

Figure 4.5 SEM image of (A) fibrous mordenite, (B) blocky clinoptilolite, and (C) grape-like clusters on clinoptilolite in sample JMK\#3@200 ${ }^{\circ} \mathrm{Ca}$................................ 57

Figure 4.6 SEM image of (A) dominant blocky clinoptilolite, (B) concoidal primary

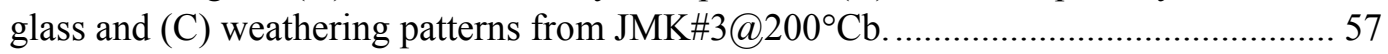

Figure 4.7 EDS Spectra of blocky crystal as seen in Figure 4.4, 10kV .................................... 58

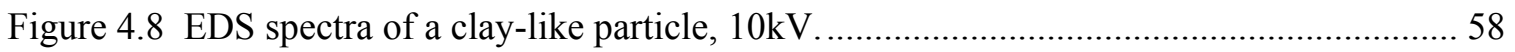

Figure 4.9 XRD spectra of reacted melt glass spectra; JMK\#3@200 Cb, JMK\#3@200Ca,

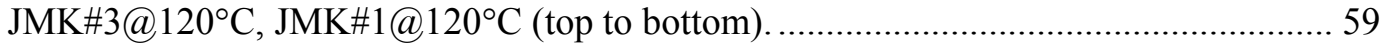

Figure 4.10 Liquid scintillation counting spectra for an activity-free standard, JMK\#1@120C supernatant, JMK\#3@120C supernatant, and JMK\#3@200C supernatant (top to bottom).

Figure 4.11 Alpha-radiography of altered nuclear melt glass samples. ...................................... 61

Figure 5.1 Map of southern and east-central Nevada showing the locations of sampling sites discussed in this report: LLNL sites (circle); USGS HAP sites (square).

Figure 5.2 Plots of mean $\delta \mathrm{D}$ vs. $\delta^{18} \mathrm{O}$ values for water samples collected from LLNL monitoring sites in central Nevada. See text for discussion.

Figure 5.3 Plot of weighted mean $\delta \mathrm{D}$ vs. $\delta^{18} \mathrm{O}$ values for cool- and warm-season precipitation samples collected from USGS HAP and LLNL central Nevada sites. GMWL $=$ global meteoric water line $\left(\delta \mathrm{D}=8 \delta^{18} \mathrm{O}+10\right)$. 


\section{List of Tables}

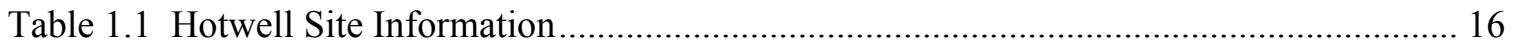

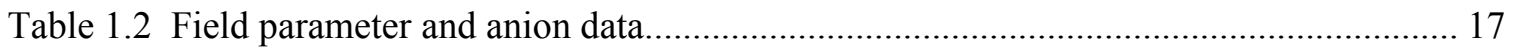

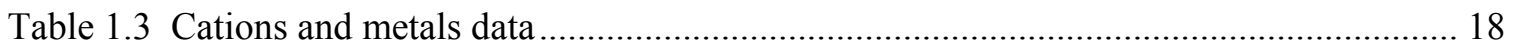

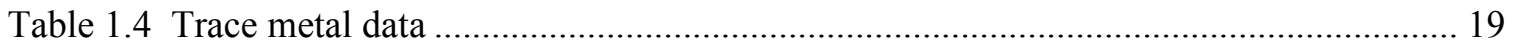

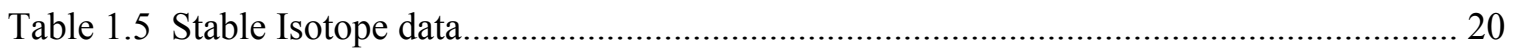

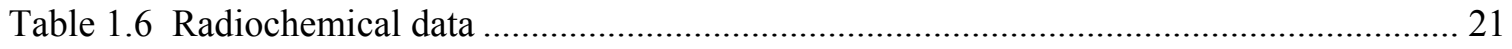

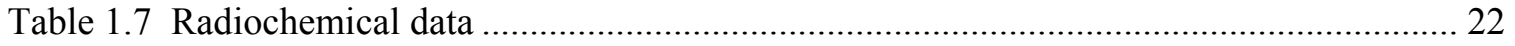

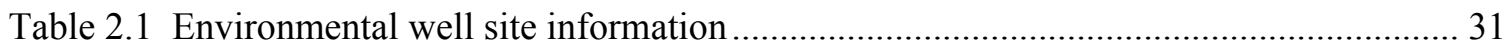

Table 2.2 Environmental well field parameter and anion data ................................................. 33

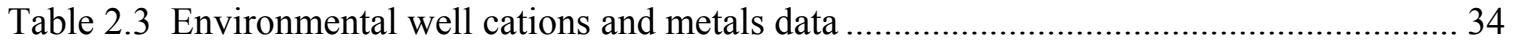

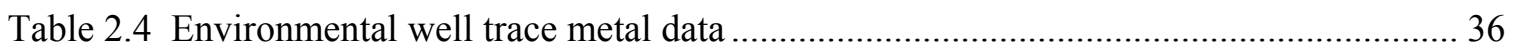

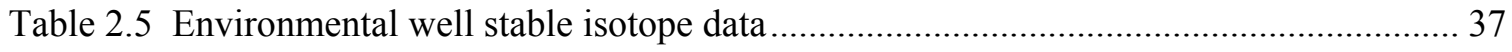

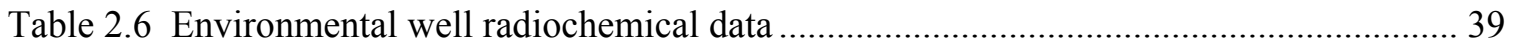

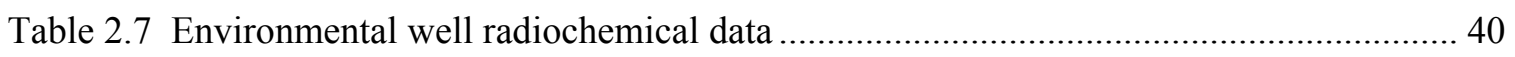

Table 3.1 Technetium and Iodine in NTS Groundwater Samples............................................. 43

Table 3.2 Technetium and Iodine Data of Solid Samples from the CHANCELLOR Test Cavity ... 46

Table 3.3 Distribution of Radionuclides in the CHANCELLOR Test Cavity .................................. 47

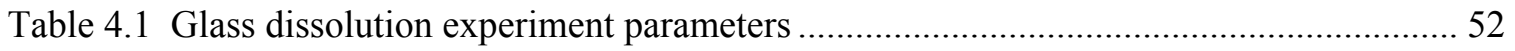

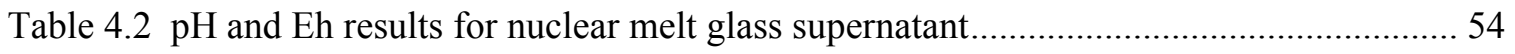

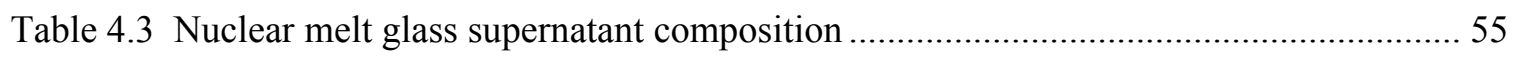

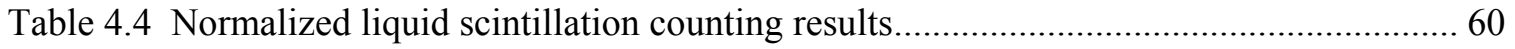

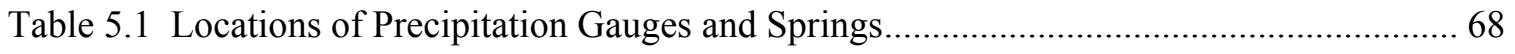

Table 5.2 Precipitation Data for LLNL Sites in Central Nevada .............................................. 70

Table 5.3 Mean Precipitation Amounts and Isotopic Values for LLNL Sites in

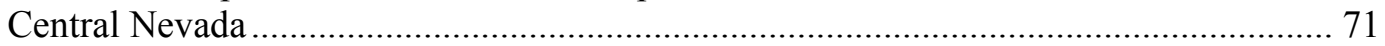

Table 5.4 Lysimeter Data for LLNL Sites in Central Nevada.................................................. 73

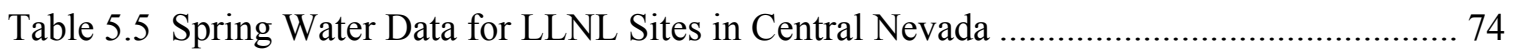

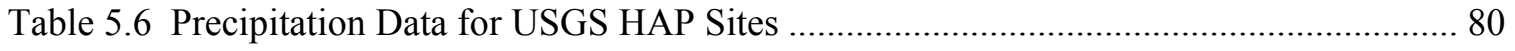

Table 5.7 Mean Precipitation Amounts and Isotopic Values for USGS HAP Sites..................... 81

Table 5.8 Comparison of Cumulative $\delta \mathrm{D}$ and Average Precipitation Data from Friedman et al. (2002) and This Study 82 


\title{
Introduction
}

\section{Hydrologic Resources Management Program and Underground Test Area Project FY 2005 Progress Report}

\author{
T.P. Rose ${ }^{1}$, A.B. Kersting ${ }^{2}$, and M. Zavarin ${ }^{1}$ \\ ${ }^{1}$ Chemical Biology and Nuclear Science Division \\ ${ }^{2}$ Glenn T. Seaborg Institute \\ Lawrence Livermore National Laboratory
}

\begin{abstract}
This report describes FY 2005 technical studies conducted by the Chemical Biology and Nuclear Science Division (CBND) at Lawrence Livermore National Laboratory (LLNL) in support of the Hydrologic Resources Management Program (HRMP) and the Underground Test Area Project (UGTA). These programs are administered by the U.S. Department of Energy, National Nuclear Security Administration, Nevada Site Office (NNSA/NSO) through the Defense Programs and Environmental Restoration Divisions, respectively. HRMP-sponsored work is directed toward the responsible management of the natural resources at the Nevada Test Site (NTS), enabling its continued use as a staging area for strategic operations in support of national security. UGTA-funded work emphasizes the development of an integrated set of groundwater flow and contaminant transport models to predict the extent of radionuclide migration from underground nuclear testing areas at the NTS.
\end{abstract}

The report is organized on a topical basis and contains five chapters that highlight technical work products produced by CBND. However, it is important to recognize that most of this work involves collaborative partnerships with the other HRMP and UGTA contract organizations. These groups include the Energy and Environment Directorate at LLNL (LLNL-E\&E), Los Alamos National Laboratory (LANL), the Desert Research Institute (DRI), the U.S. Geological Survey (USGS), Stoller-Navarro Joint Venture (SNJV), and Bechtel Nevada (BN).

Chapter 1 is a summary of FY 2005 sampling efforts at NTS near-field "hot" wells, and presents new chemical and isotopic data for groundwater samples from four near-field wells. These include ER-20-5\#1 and ER-20-5\#3, U-3cn PS\#2 (BILBY), and UE-2ce (NASH).

Chapter 2 is a summary of the results of chemical and isotopic measurements of groundwater samples from three UGTA environmental monitoring wells. These include an existing well ER-12-1 and two new wells ER-12-3 and ER-12-4 all located in Area 12 on Rainier Mesa. 
Chapter 3 is a summary of the distribution of ${ }^{99} \mathrm{Tc}$ and ${ }^{129} \mathrm{I}$ in the vicinity of underground nuclear tests at the NTS, with particular focus on the recent drillback samples collected at CHANCELLOR in FY2005. This work was presented as part of the Methods and Applications of Radioanalytical Chemistry (MARC VII) conference held in Kailua-Kona, Hawai' $i$, USA, April $3-7$, 2006. Proceedings are published in the Journal of Radioanalytical and Nuclear Chemistry.

Chapter 4 is a summary of the results of long term nuclear melt glass alteration experiments conducted at 120 and $200^{\circ} \mathrm{C}$. The secondary mineral phases and associated water from the nuclear melt glass dissolution experiments were characterized using $\mathrm{Eh} / \mathrm{pH}$, ion chromatography (IC), inductively coupled plasma mass spectrometry (ICPMS), Liquid Scintillation Counting (LSC), Scanning Electron Microscopy (SEM), Energy Dispersive X-ray Spectroscopy (EDS), X-ray Diffraction (XRD), and $\alpha$ radiography. The nuclear melt glass sequesters the majority of the long-lived radionuclides and alteration of this glass contributes to the hydrologic source term.

Chapter 5 presents stable isotope data from precipitation, spring discharge, and soil water at four sites in central Nevada. Stable isotope measurements at the USGS High Altitude Precipitation (HAP) network in eastern Nevada are also discussed. The data from these sites can be used to improve our understanding of precipitation and recharge processes in Nevada, and can be applied to the development of conceptual models of hydrologic processes, for validating numerical groundwater flow models, and for monitoring potential changes in climate over time. 


\title{
Chapter 1
}

\section{FY 2005 Hot Well Data}

\author{
G.F. Eaton, V.Genetti, Q. (Max) Hu, G.B. Hudson, R.E. Lindvall, J.E. Moran, E.C. \\ Ramon, T.P. Rose, R.W. Williams, M. Zavarin, and P. Zhao \\ Chemical Biology and Nuclear Science Division \\ Lawrence Livermore National Laboratory
}

\subsection{Introduction}

This chapter summarizes the results of chemical and isotopic analyses of groundwater samples collected from near-field "hot" wells at the NTS during FY 2005. This work is the latest contribution to a long-standing effort aimed at understanding radionuclide transport processes at the NTS. These data are required in the development and verification of contaminant transport models for the Underground Test Area (UGTA) project. Collaborating agencies in the hot well sampling effort include Los Alamos National Laboratory (LANL), the U.S. Geological Survey (USGS), the Desert Research Institute (DRI), Bechtel Nevada (BN), and Stoller-Navarro Joint Venture (SNJV).

Groundwater samples were collected from four NTS near-field wells during FY 2005. These include ER-20-5\#1 and ER-20-5\#3 (both located 280 meters southwest of TYBO and $\sim 1.3$ kilometeres south of BENHAM), U-3cn PS\#2a (perforations located $\sim 200$ meters above the BILBY working point), and UE-2ce (located 180 meters south of NASH). Figure 1.1 shows the locations of these wells at the NTS. Laboratory analytical protocols are fully described in the LLNL Standard Operating Procedures written in support of the UGTA Project (LLNL, 2004). Tables 1.1 through 1.7 contain the analytical results for the FY 2005 samples together with comparative data for samples collected over the past several years. Significant features of the FY 2005 data are highlighted in the sections that follow. 


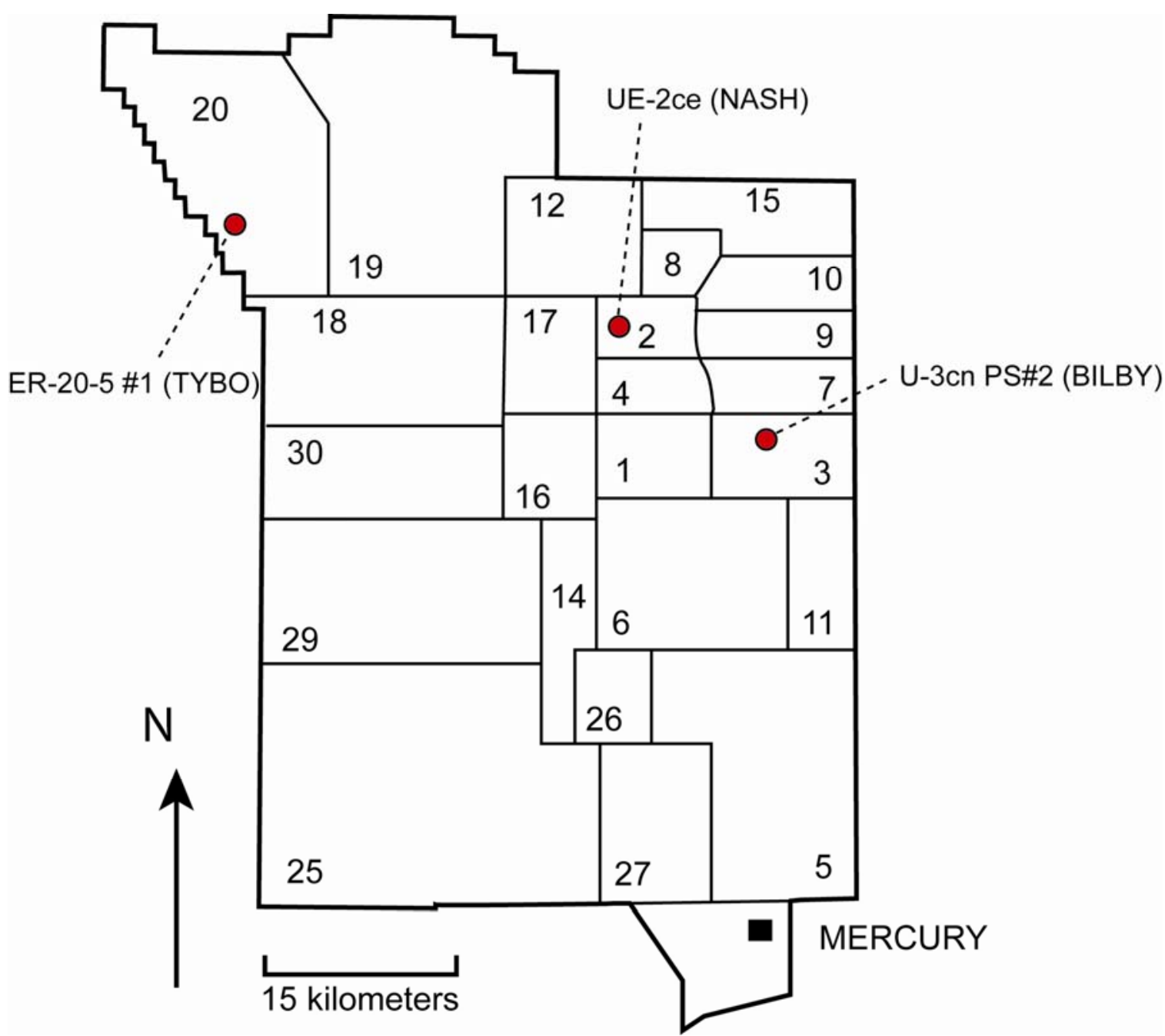

Figure 1.1 Map of the NTS showing Hotwell sampling locations for FY2005.

\subsection{ER-20-5 \#1}

The ER-20-5 well cluster consists of two near-field monitoring wells (\#1 and \#3) located on Pahute Mesa, in Area 20 of the NTS. The wellhead for ER-20-5 \#1 is situated less than 300 meters from surface ground zero of the TYBO underground nuclear test, which was detonated in the U-20y emplacement hole on May 14, 1975. The production interval for ER-20-5\#1 is 701 to 784 meters below ground surface in Tertiary rhyolite of the Topopah Spring Tuff (Figure 1.2).

Groundwater characterization samples were collected from well ER-20-5 \#1 on November 30, 2004. Approximately 29,340 gallons of water were pumped from the well prior to sample collection. Prior to 2004, ER-20-5\#1 was last sampled on July 9, 1998 (LLNL, 1998). Since that time, there have been no significant changes in the chemical and isotopic characteristics of the groundwater. 


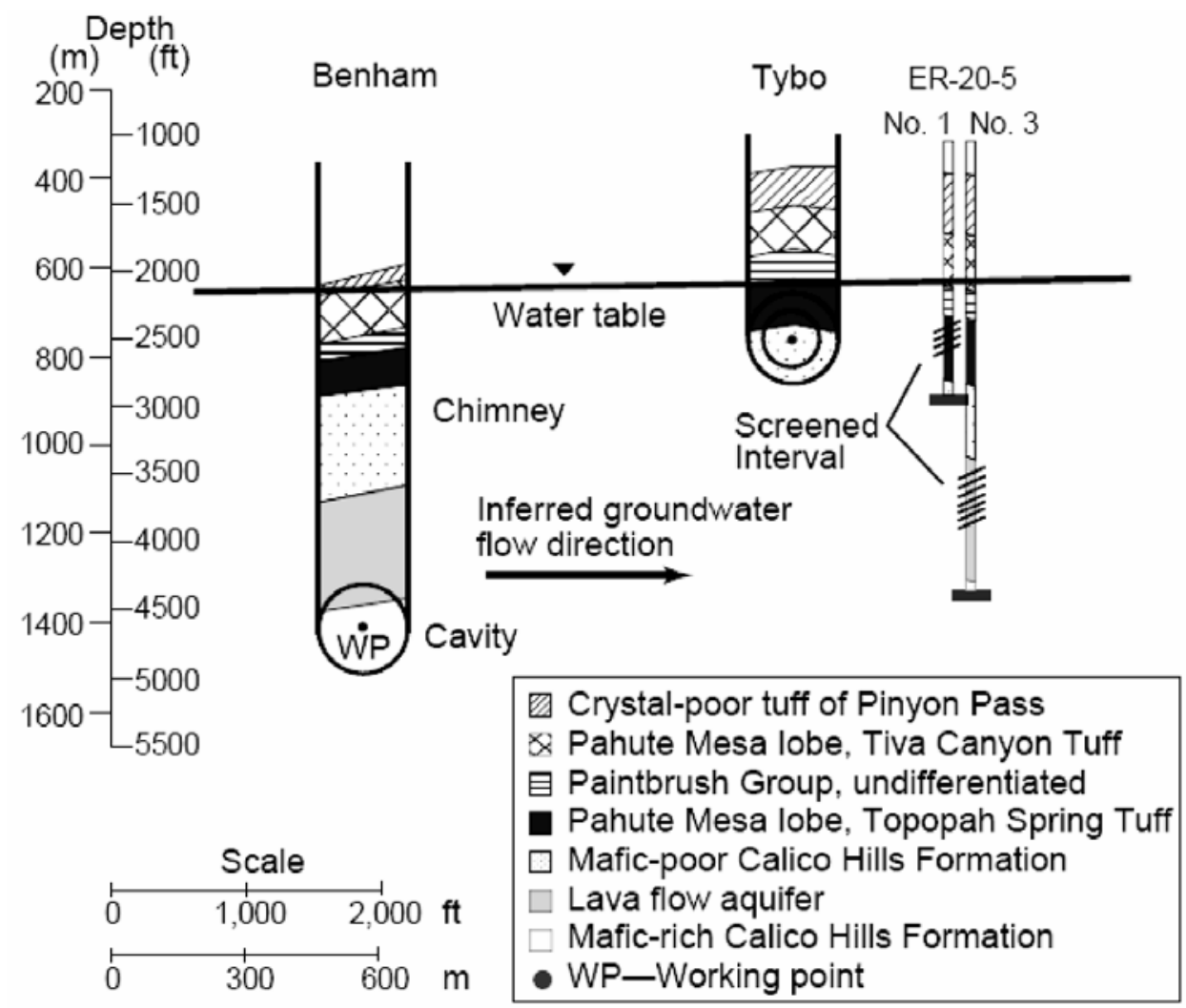

Figure 1.2 Location of the ER-20-5 well cluster relative to the TYBO and BENHAM tests. From Kersting et al. (1999). Cavity size is not to scale.

Well ER-20-5 \#1 produces $\mathrm{Na}-\mathrm{HCO}_{3}$ groundwater with modest enrichments in chloride and sulfate anions. The well is located near the convergence of two separate groundwater flow systems that underlie Pahute Mesa (Rose et al., 2002). Groundwater to the east of this location is characterized by very low chloride and sulfate values (e.g. Water Well 20) whereas groundwater immediately to the west has much higher concentrations of these anions (e.g. well ER-EC-1). The groundwater from ER-20-5 \#1 appears to contain a mixture of these two water types. The stable isotope composition of ER-20-5 \#1 groundwater $\left(\delta \mathrm{D}=-115 \%\right.$ and $\delta^{18} \mathrm{O}=-14.9 \%$ ) is consistent with this interpretation.

The tritium activity in ER-20-5 \#1 groundwater was $3.8 \times 10^{7} \mathrm{pCi} / \mathrm{L}$ at the time of sampling, as determined by liquid scintillation counting (LSC). The decay corrected value at time zero for the TYBO test is $2.0 \times 10^{8} \mathrm{pCi} / \mathrm{L}$. This is in good agreement with the decay corrected tritium activity in the samples collected in $1998\left(2.3 \times 10^{8} \mathrm{pCi} / \mathrm{L}\right)$. Kersting et al. (1999) showed that plutonium in ER-20-5 groundwater originates from the BENHAM underground test, which is located approximately $1.3 \mathrm{~km}$ north of the well cluster. It is uncertain whether all of the radionuclides that are observed at the ER-20-5 \#1 well originated from BENHAM, or if some fraction of the more mobile species (such as tritium) may have originated from the TYBO test. It is notable that the tritium activity in ER-20-5 \#1 is substantially higher than that of the deeper ER-20-5 \#3 well. The 
radioactivity encountered in the deeper well is more likely to have a direct source from BENHAM due to the presence of low permeability rocks between the two aquifers where the \#1 and \#3 wells are completed; this low permeability rock would inhibit downward leakage of contaminated groundwater from the TYBO test cavity.

Dissolved inorganic carbon (DIC) in ER-20-5 \#1 groundwater has a ${ }^{14} \mathrm{C}$ value of $9.6 \times 10^{4}$ percent modern carbon (pmc), approximately three orders of magnitude higher than the modern atmospheric radiocarbon concentration. At the measured DIC concentration of $193 \mathrm{mg} / \mathrm{L}$, this corresponds to a dissolved ${ }^{14} \mathrm{C}$ activity of $224 \mathrm{pCi} / \mathrm{L}$. The relatively high $\delta^{13} \mathrm{C}$ value of the water $(-4.7 \%$ o) suggests it has largely equilibrated with fracture-lining calcite mineralization that is widely observed in the Pahute Mesa aquifers (Benedict et al., 2000).

${ }^{36} \mathrm{Cl}$ is a long-lived neutron activation product $\left(\mathrm{t}_{1 / 2}=3 \times 10^{5}\right.$ years $)$ that is produced during nuclear detonations, and is highly mobile as the soluble chloride anion. ER-20-5 \#1 groundwater has a ${ }^{36} \mathrm{Cl} / \mathrm{Cl}$ ratio $\left(4.4 \times 10^{-9}\right)$ that is four orders of magnitude above the modern atmospheric ratio for southern Nevada $\left(\sim 5 \times 10^{-13}\right.$, Fabryka-Martin et al., 1993). Due to its long half-life, the total ${ }^{36} \mathrm{Cl}$ activity in the ER-20-5 \#1 groundwater is fairly low $(3.6 \mathrm{pCi} / \mathrm{L})$. This value is almost unchanged from the previous measurement in 1998 (3.3 pCi/L).

The fission products ${ }^{99} \mathrm{Tc}$ and ${ }^{129} \mathrm{I}$ were measured in ER-20-5 \#1 groundwater for the first time in 2004. Technetium and iodine are both highly mobile as soluble anions under oxidizing conditions. For comparative purposes, the ${ }^{99} \mathrm{Tc}$ and ${ }^{129} \mathrm{I}$ concentrations in archival samples collected in 1998 were also analyzed. The measured ${ }^{99} \mathrm{Tc}$ activity was $0.35 \mathrm{pCi} / \mathrm{L}$ in the 2004 samples and $0.27 \mathrm{pCi} / \mathrm{L}$ in the 1998 samples. The ${ }^{129} \mathrm{I}$ activity is of a similar magnitude, with values of $0.19 \mathrm{pCi} / \mathrm{L}$ in 2004 and $0.27 \mathrm{pCi} / \mathrm{L}$ in 1998 . The small differences between the 1998 and 2004 results are probably due to analytical uncertainties.

ER-20-5 \#1 groundwater has a ${ }^{87} \mathrm{Sr} /{ }^{86} \mathrm{Sr}$ ratio of 0.71047 , and a $\delta^{87} \mathrm{Sr}$ value of $1.79 \%$. This is consistent with the range of previously reported values for groundwater from the volcanic aquifers in the NTS region (Peterman et al., 1993; Thomas et al., 2002). The concentration of dissolved uranium $(14.1 \mu \mathrm{g} / \mathrm{L})$ is elevated compared with most NTS groundwaters (Paces et al., 2002) but the ${ }^{235} \mathrm{U} /{ }^{238} \mathrm{U}$ ratio indicates the uranium is natural in origin. Elevated uranium concentrations are not unusual in some volcanic tuffs, and we infer that the high uranium content in the ER-20-5 \#1 groundwater reflects the dissolution of uranium-bearing minerals present in the host rock. The ${ }^{234} \mathrm{U} /{ }^{238} \mathrm{U}$ activity ratio (2.90) reveals a modest enrichment in dissolved ${ }^{234} U$ due to preferential leaching of this isotope from the aquifer matrix following $\alpha$-decay of ${ }^{238} \mathrm{U}$.

Plutonium is present at low concentrations $(6.4 \mathrm{pg} / \mathrm{L})$ in the 2004 groundwater samples, with a combined ${ }^{239,240} \mathrm{Pu}$ activity of $0.42 \mathrm{pCi} / \mathrm{L}$. The observed plutonium activity is similar to previously reported values, and is strongly associated with the colloid fraction of the groundwater. As noted above, Kersting et al. (1999) demonstrated that the plutonium in ER-20-5 groundwater originated from the BENHAM test. 


\section{$1.3 \quad$ ER-20-5 \#3}

The ER-20-5 well cluster consists of two near-field monitoring wells (\#1 and \#3) located in the western part of Area 20, on the NTS. Well ER-20-5 \#3 is situated less than 300 meters from surface ground zero of the TYBO underground nuclear test, which was detonated in the U-20y emplacement hole on May 14, 1975. The well was completed to a total depth of 1,309 meters below ground surface in rhyolitic lavas of the Calico Hills Formation, with a single slotted perforation interval between 1,046 and 1,183 meters (Figure 1.2).

Groundwater characterization samples were collected from ER-20-5 \#3 on November 29, 2004. Prior to 2004, ER-20-5 \#3 was last sampled on November 15, 2001 (Rose et al., 2003). No significant changes in the chemical and isotopic characteristics of the water were observed between these two sets of samples.

ER-20-5 \#3 produces sodium bicarbonate groundwater with modest levels of $\mathrm{Cl}^{-}$and $\mathrm{SO}_{4}{ }^{2-}$ that is characteristic of the tuffaceous volcanic rocks beneath Pahute Mesa. The stable isotope composition of the groundwater $\left(\delta \mathrm{D}=-114 \%\right.$ and $\delta^{18} \mathrm{O}=-15.1 \%$ o $)$ is similar to that produced from other wells in this area. The combined water quality and stable isotope data suggest that the ER-20-5 well cluster contains a mixture of groundwater from two distinct regional flow systems that underlie Pahute Mesa. These flow systems are characterized by modest differences in stable isotope values and significantly different concentrations of chloride and sulfate (Rose et al., 2002).

The tritium activity in ER-20-5 \#3 groundwater was $1.13 \times 10^{5} \mathrm{pCi} / \mathrm{L}$ at the time of sampling, as determined by liquid scintillation counting (LSC). The decay-corrected value at time zero for the TYBO test $\left(6.0 \times 10^{5} \mathrm{pCi} / \mathrm{L}\right)$ is very similar to the decay-corrected value measured in $2001\left(6.3 \times 10^{5} \mathrm{pCi} / \mathrm{L}\right)$. This implies that radionuclide concentrations in the water have remained fairly constant over this time period. Despite the close proximity of the ER-20-5 well cluster to the TYBO test, plutonium isotope measurements indicate that the BENHAM test was the source of the Pu activity in both of the ER-20-5 wells (Kersting et al., 1999). Given that the BENHAM test is located $>1 \mathrm{~km}$ up gradient from the ER-20-5 well cluster, these results have important implications for the long-range transport of radionuclides in fracture-dominated flow systems.

Dissolved inorganic carbon (DIC) in ER-20-5 \#3 groundwater has a ${ }^{14} \mathrm{C}$ value of $1.68 \times 10^{3} \mathrm{pmc}$, and a corresponding ${ }^{14} \mathrm{C}$ activity of $2.7 \mathrm{pCi} / \mathrm{L}$. This is similar to the activity measured on samples collected in $2001(2.1 \mathrm{pCi} / \mathrm{L})$. The $\delta^{13} \mathrm{C}$ value measured in $2004(-9.3 \%$ ) is more than 4\%o lighter than reported in November 2001. It is unlikely that this represents any change in the source of DIC in this aquifer and is more likely the result of analytical error.

ER-20-5 \#3 groundwater has a ${ }^{36} \mathrm{Cl} / \mathrm{Cl}$ ratio $\left(2.27 \times 10^{-11}\right)$ that is $\sim 45$ times the modern atmospheric ratio for southern Nevada. Although this ratio is well above environmental levels, the calculated ${ }^{36} \mathrm{Cl}$ activity in the groundwater is fairly low $(0.013 \mathrm{pCi} / \mathrm{L})$. Low activities were also observed for the fission products ${ }^{99} \mathrm{Tc}$ and ${ }^{129} \mathrm{I}$, both of which are transported as soluble anions under oxidizing conditions. The measured ${ }^{99} \mathrm{Tc}$ activity is 
$0.017 \mathrm{pCi} / \mathrm{L}$, while the ${ }^{129} \mathrm{I}$ activity is $0.0014 \mathrm{pCi} / \mathrm{L}$. In general, these data indicate substantial dilution of the radionuclide source term compared with values typically observed in cavity fluid samples. Nevertheless, it is evident that a variety of radionuclides can be transported at low concentrations over fairly large distances.

The groundwater from ER-20-5 \#3 has a ${ }^{87} \mathrm{Sr} /{ }^{86} \mathrm{Sr}$ ratio of 0.70841 and a $\delta^{87} \mathrm{Sr}$ value of $-1.11 \%$. These results are fairly typical of the values found in groundwater from the NTS volcanic aquifers (Peterman et al., 1993; Thomas et al., 2002). The concentration of dissolved uranium $(5.6 \mu \mathrm{g} / \mathrm{L})$ is within the normal range of values for groundwater from the NTS volcanic units (Paces et al., 2002), and the ${ }^{235} \mathrm{U} /{ }^{238} \mathrm{U}$ ratio indicates the uranium is natural in origin. The ${ }^{234} \mathrm{U} /{ }^{238} \mathrm{U}$-activity ratio (2.53) indicates a moderate enrichment in ${ }^{234} \mathrm{U}$ due to preferential leaching of this isotope from the aquifer matrix following $\alpha$ decay of ${ }^{238} \mathrm{U}$.

The ER-20-5 \#3 samples were analyzed for plutonium, but the measured concentration was very close to the method detection limit $(0.6 \mathrm{pg} / \mathrm{L})$ and it was not possible to obtain good quality isotope ratio data. Kersting et al. (1999) previously observed plutonium in ER-20-5 \#3, but pre-concentration of large amounts of colloidal material was necessary to obtain reliable isotope ratio measurements. 


\subsection{U-3cn PS\#2 (BILBY)}

U-3en PS\#2 is located in the northwestern part of Area 3 of the NTS. The well head is located less than $300 \mathrm{~m}$ from surface ground zero of the BILBY underground nuclear test (249 kt announced yield), which was detonated under Yucca Flat in the U-3cn emplacement hole on September 13, 1963 (DOE, 2000). The BILBY test was the first underground nuclear test detonated below the water table at the NTS (Buddemeier and Isherwood, 1985).

U-3cn PS\#2 was drilled into the chimney of the BILBY test, and originally perforated the test cavity (W.P. $=713 \mathrm{~m})$. However, the pipe was crimped at $587 \mathrm{~m}$ shortly after completion, probably due to shifting blocks within the chimney (Garber, 1971), and the production interval is now between 512 and $527 \mathrm{~m}$ below ground surface, where saturated rocks consist of Tertiary-age rhyolitic tuffs of the Indian Trail Formation (Figure 1.3).

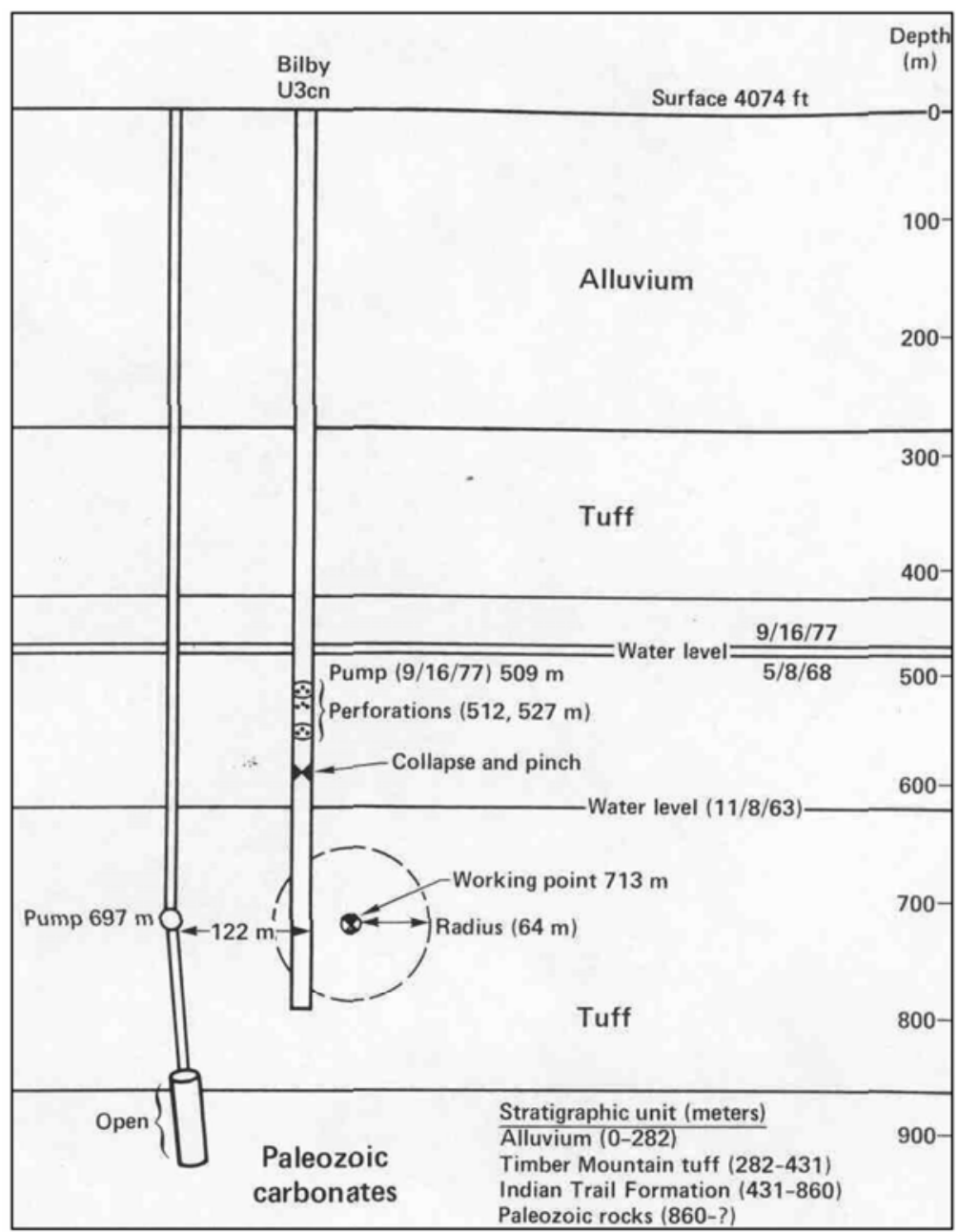

Figure 1.3 The BILBY near-field. From Buddemeier and Isherwood (1985). 
Groundwater characterization samples were collected from well U-3cn PS\#2 on December 9, 2004. Prior to sampling, a cumulative purge volume of $\sim 1.5 \times 10^{4}$ gallons of water was pumped from the well. The well produces a sodium-potassium-bicarbonate groundwater that is low in $\mathrm{Cl}$ and $\mathrm{SO}_{4}$, and is typical of the water found in tuffaceous volcanic rocks beneath Yucca Flat (Winograd and Thordarson, 1975). U-3cn PS\#2 groundwater has stable isotope $\left(\delta \mathrm{D}\right.$ and $\left.\delta^{18} \mathrm{O}\right)$ values that are depleted in heavy isotopes relative to modern precipitation in Yucca Flat (Ingraham et al., 1991) and may reflect water that was recharged during cooler, wetter climatic conditions that were prevalent in this area during late Pleistocene time (e.g. Claassen, 1986).

The tritium activity of the U-3en PS\#2 groundwater, measured at LLNL by liquid scintillation counting (LSC) following sampling, was $7.9 \times 10^{6} \mathrm{pCi} / \mathrm{L}$. The decay corrected value at time zero for the BILBY test is $8.0 \times 10^{7} \mathrm{pCi} / \mathrm{L}$. This is in good agreement with the decay corrected tritium activity in the samples collected in $2001\left(8.6 \times 10^{7} \mathrm{pCi} / \mathrm{L}\right)$ and $1997\left(8.4 \times 10^{7} \mathrm{pCi} / \mathrm{L}\right)$.

Dissolved inorganic carbon (DIC) in U-3cn PS\#2 groundwater has a ${ }^{14} \mathrm{C}$ value of $1.15 \times 10^{5} \mathrm{pmc}$, approximately three orders of magnitude higher than the modern atmospheric radiocarbon concentration. At the measured DIC concentration of $269 \mathrm{mg} / \mathrm{L}$, this corresponds to a dissolved ${ }^{14} \mathrm{C}$ activity of $372 \mathrm{pCi} / \mathrm{L}$. The $\delta^{13} \mathrm{C}$ value of the water was $-7.0 \%$, a little over $3 \%$ lighter than 2001 .

${ }^{36} \mathrm{Cl}$ is a long-lived neutron activation product $\left(\mathrm{t}_{1 / 2}=3 \times 10^{5}\right.$ years $)$ that is produced during nuclear detonations, and is highly mobile as the soluble chloride anion. U-3cn PS\#2 groundwater has a ${ }^{36} \mathrm{Cl} / \mathrm{Cl}$ ratio $\left(4.5 \times 10^{-8}\right)$ that is five orders of magnitude above the modern atmospheric ratio for southern Nevada $\left(\sim 5 \times 10^{-13}\right.$, Fabryka-Martin et al., 1993). The total ${ }^{36} \mathrm{Cl}$ activity in the U-3cn PS\#2 groundwater is $25.5 \mathrm{pCi} / \mathrm{L}$, less than the previous measurement in $2001(43.2 \mathrm{pCi} / \mathrm{L})$. The ${ }^{36} \mathrm{Cl}$ activity in $\mathrm{U}-3 \mathrm{cn} \mathrm{PS} \# 2$ groundwater is among the highest observed at the NTS.

The fission products ${ }^{99} \mathrm{Tc}$ and ${ }^{129} \mathrm{I}$ were measured in U-3cn PS\#2 groundwater by ICP-MS and AMS, respectively. Technetium and iodine are both highly mobile as soluble anions under oxidizing conditions. For comparative purposes, the ${ }^{99} \mathrm{Tc}$ and ${ }^{129} \mathrm{I}$ concentrations in archival samples collected in 2001 were also analyzed. The measured ${ }^{99}$ Tc activity was $62.6 \mathrm{pCi} / \mathrm{L}$ in the 2004 samples and $82.6 \mathrm{pCi} / \mathrm{L}$ in the 2001 samples. The ${ }^{129} \mathrm{I}$ activity in the samples was $0.25 \mathrm{pCi} / \mathrm{L}$ in 2004 and $0.16 \mathrm{pCi} / \mathrm{L}$ in 2001 . There is no consistent increase or decrease in test-derived radionuclide tracer $\left({ }^{3} \mathrm{H},{ }^{36} \mathrm{Cl},{ }^{99} \mathrm{Tc}\right.$, and $\left.{ }^{129} \mathrm{I}\right)$ concentrations between 2001 and 2004. Thus, changes in radionuclide tracer activities are not likely to be the result of physical transport processes.

U-3en PS\#2 groundwater has a ${ }^{87} \mathrm{Sr} /{ }^{86} \mathrm{Sr}$ ratio of 0.70984 , and a $\delta^{87} \mathrm{Sr}$ value of $0.9 \%$. This is consistent with the range of previously reported values for groundwater from the volcanic aquifers in the NTS region (Peterman et al., 1993; Thomas et al., 2002). The concentration of dissolved uranium $(10.3 \mu \mathrm{g} / \mathrm{L})$ is elevated compared with most NTS groundwaters (Paces et al., 2002) but the ${ }^{235} \mathrm{U} /{ }^{238} \mathrm{U}$ ratio indicates the uranium is natural in origin. Elevated uranium concentrations are not unusual in some volcanic tuffs, and we infer that the high uranium content in the U-3cn PS\#2 groundwater reflects the 
dissolution of uranium-bearing minerals present in the host rock. The ${ }^{234} \mathrm{U} /{ }^{238} \mathrm{U}$ activity ratio (2.80) reveals a modest enrichment in dissolved ${ }^{234} \mathrm{U}$ due to preferential leaching of this isotope from the aquifer matrix following $\alpha$-decay of ${ }^{238} \mathrm{U}$.

Plutonium was not detected in the groundwater from U-3cn PS\#2 $\left({ }^{239,240} \mathrm{Pu}<0.01 \mathrm{pCi} / \mathrm{L}\right)$. Plutonium was measured on a bulk sample which includes both the colloidal and aqueous fractions of the groundwater.

\subsection{UE-2ce (NASH)}

UE-2ce is located in Area 2 of the NTS and lies at a relatively high elevation on the eastern rim of Yucca Flat. UE-2ce was completed on January 25, 1977 to a depth of 503 meters. It is located less than $183 \mathrm{~m}$ south from NASH surface ground zero. The NASH test was detonated in emplacement hole U-2ce at a depth of 368 meters on 19 January 1967 with an announced yield of $39 \mathrm{kt}$ (DOE, 2000). Both the working point of the NASH test and the UE-2ce satellite well perforations are in the Paleozoic carbonate of the Lower Carbonate Aquifer (LCA). The cavity of the NASH test is entirely above the water table (cavity radius is 29.1 meters). However, ${ }^{3} \mathrm{H}$ has been consistently measured at the satellite well. The boundary between the LCA and the overlying volcanic tuff is at $340 \mathrm{~m}$-bgs (Buddemeier \& Isherwood, 1985). Near-field geometry and water level measurements at UE-2ce are plotted in Figure 1.4. 


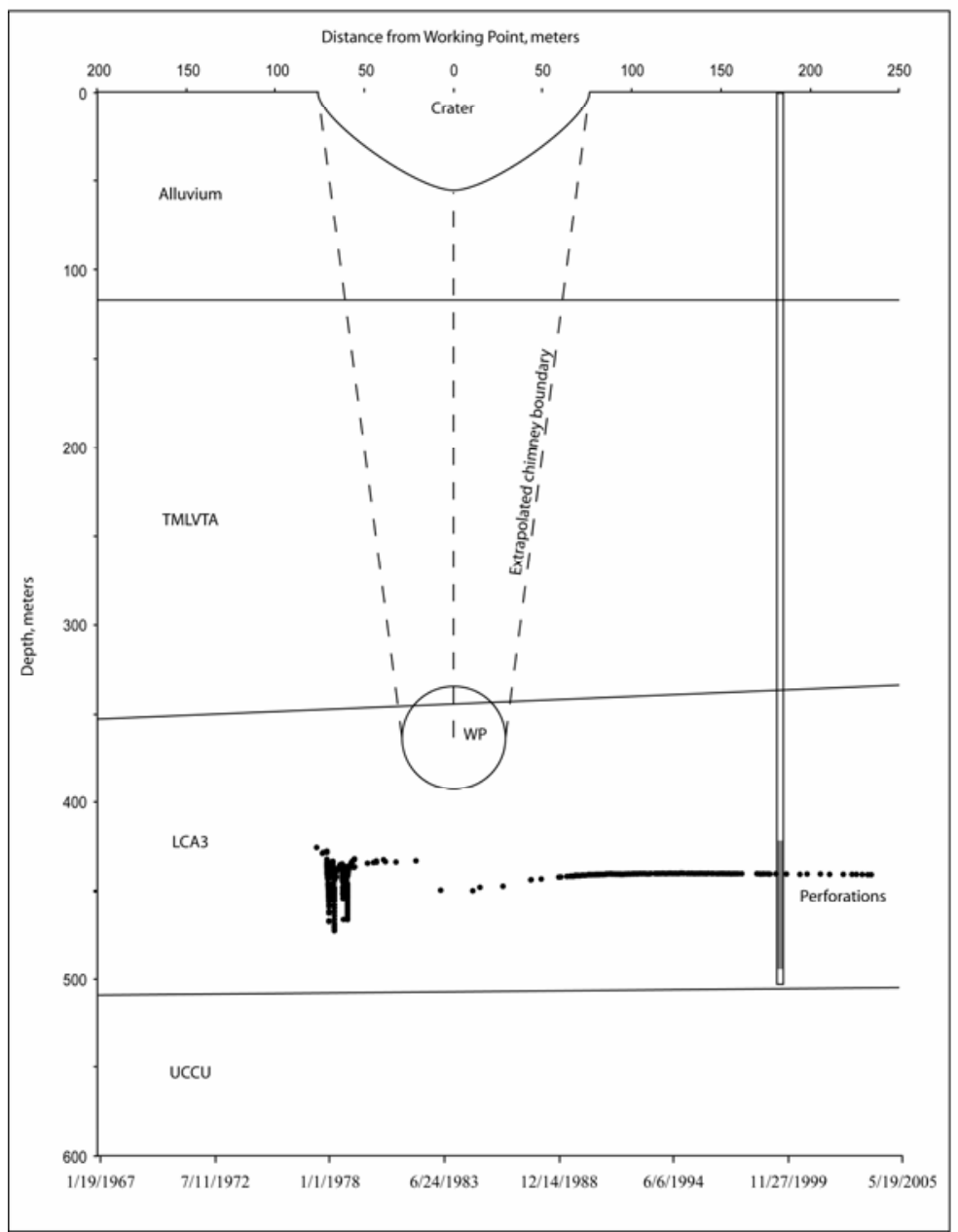

Figure 1.4 The NASH near-field geometry and water level measurements as a function of time.

Groundwater characterization samples were collected from well UE-2ce using evacuated steel pressure tubes, lowered into the hole with the USGS wire-line bailer, between July 11 and 13, 2005. Even though these samples were collected over a three day period, all were collected at the same depth (481 meters), and a common date of 12 July 2005 was assigned to all analytical samples collected during this period. The water depth at the time of sampling was approximately 441 meters, indicating that the water level has not drastically changed since the well was last sampled in August of 2001 ( 448 meters). Importantly, it should be noted that the water level in UE-2ce has never returned to the levels measured soon after well completion and before significant pumping in the 1980s (Figure 1.4).

Well UE-2ce produces a Ca-Mg-Na- $\mathrm{HCO}_{3}$ type groundwater that is low in $\mathrm{Cl}$ and $\mathrm{SO}_{4}$, and is similar to other LCA waters (SNJV, 2006). UE-2ce has $\delta^{18} \mathrm{O}$ and $\delta \mathrm{D}$ values that are enriched in heavy isotopes $\left(\delta^{18} \mathrm{O}=-12.6 \%\right.$; $\delta \mathrm{D}=-98 \%$ ) compared with most Yucca Flat waters, particularly those from the LCA. The data suggest that a significant fraction of the groundwater at UE-2ce may be derived from local recharge (SNJV, 2006). 
The tritium activity of the UE-2ce groundwater, measured at LLNL by liquid scintillation counting (LSC), was $9.3 \times 10^{4} \mathrm{pCi} / \mathrm{L}$. The decay corrected value at time zero for the NASH test is $8.3 \times 10^{5} \mathrm{pCi} / \mathrm{L}$. The decay corrected tritium activity for samples collected in 2001 was $9.9 \times 10^{5} \mathrm{pCi} / \mathrm{L}$. It should be noted that the ${ }^{3} \mathrm{H}$ activities found in these bailed samples are much lower ( $\sim 2$ orders of magnitude) than in pumped samples collected in 1984 (Buddemeier and Isherwood, 1985).

Dissolved inorganic carbon (DIC) in UE-2ce groundwater has a measured DIC concentration of $337 \mathrm{mg} / \mathrm{L}$ and a ${ }^{14} \mathrm{C}$ value of $2.0 \times 10^{2} \mathrm{pmc}$ that corresponds to a dissolved ${ }^{14} \mathrm{C}$ activity of $0.82 \mathrm{pCi} / \mathrm{L}$. Though the ${ }^{14} \mathrm{C}$ activity is low, it appears to be testderived and suggests that ${ }^{14} \mathrm{C}$ transport from NASH to the satellite well has taken place. The $\delta^{13} \mathrm{C}$ value of the water was $-6.6 \%$, about $1 \%$ lighter than 2001 . Nevertheless, both values are indicative of dissolution of LCA rock to produce $\delta^{13} \mathrm{C}$ values intermediate between soil zone $\delta^{13} \mathrm{C}$ and LCA rock $\delta^{13} \mathrm{C}$.

${ }^{36} \mathrm{Cl}$ is a long-lived neutron activation product $\left(\mathrm{t}_{1 / 2}=3 \times 10^{5}\right.$ years $)$ that is produced during nuclear detonations, and is highly mobile as the soluble chloride anion. UE-2ce groundwater has a ${ }^{36} \mathrm{Cl} / \mathrm{Cl}$ ratio of $9.54 \times 10^{-10}$ that is three orders of magnitude above the modern atmospheric ratio for southern Nevada $\left(\sim 5 \times 10^{-13}\right.$, Fabryka-Martin et al., 1993). The total ${ }^{36} \mathrm{Cl}$ activity in the U-3cn PS\#2 groundwater is $0.45 \mathrm{pCi} / \mathrm{L}$, slightly less than the previous measurement in $2001(0.83 \mathrm{pCi} / \mathrm{L})$. However, both values are indicative of a test-derived source and suggest ${ }^{36} \mathrm{Cl}$ transport from NASH to the satellite well.

The fission products ${ }^{99} \mathrm{Tc}$ and ${ }^{129} \mathrm{I}$ were measured in UE-2ce groundwater by ICP-MS and AMS, respectively. Technetium and iodine are both highly mobile as soluble anions under oxidizing conditions. The measured ${ }^{99} \mathrm{Tc}$ activity was $<2.4 \times 10^{-3} \mathrm{pCi} / \mathrm{L}$. The ${ }^{129} \mathrm{I}$ activity was $0.024 \mathrm{pCi} / \mathrm{L}$. The ${ }^{129} \mathrm{I}$ value is similar to that measured in 2001 $(0.032 \mathrm{pCi} / \mathrm{L})$. There is no consistent increase or decrease in radionuclide activities between 2001 and 2005.

UE-2ce groundwater has a ${ }^{87} \mathrm{Sr} /{ }^{86} \mathrm{Sr}$ ratio of 0.71137 , a $\delta^{87} \mathrm{Sr}$ value of $3.1 \%$, and a $\mathrm{Sr}$ concentration of $194 \mu \mathrm{g} / \mathrm{L} .{ }^{1}$ This is consistent with the range of previously reported values for groundwater from the carbonate aquifer in Yucca Flat. However, both the $\mathrm{Sr}$ isotope ratio and $\mathrm{Sr}$ concentration are on the low end for LCA waters and suggest some interaction with volcanic aquifer rocks. The concentration of dissolved uranium $(2.0 \mu \mathrm{g} / \mathrm{L})$ is consistent with most NTS groundwaters (Paces et al., 2002) and the ${ }^{235} \mathrm{U}{ }^{238} \mathrm{U}$ ratio indicates the uranium is natural in origin. The ${ }^{234} \mathrm{U} /{ }^{238} \mathrm{U}$ activity ratio (3.53) reveals a modest enrichment in dissolved ${ }^{234} \mathrm{U}$ due to preferential leaching of this isotope from the aquifer matrix following $\alpha$-decay of ${ }^{238} \mathrm{U}$.

Plutonium was not detected in the groundwater from UE-2ce $\left({ }^{239,240} \mathrm{Pu}<0.07 \mathrm{pCi} / \mathrm{L}\right)$. The colloidal and aqueous fractions were measured by pre-concentrating 3 liters of sample. $\mathrm{Pu}$ was not detected in either fraction.

\footnotetext{
${ }^{1}$ The value has been corrected since it was first reported in the UE-2ce well report dated July 14, 2006.
} 


\subsection{References}

Benedict, F.C., Jr., Rose, T.P., and Zhou, X. (2000) Mineralogical, chemical, and isotopic characterization of fracture-coating minerals in borehole samples from western Pahute Mesa and Oasis Valley, Nevada. Lawrence Livermore National Laboratory UCRL-ID-152919, September 2000, 111 p.

Buddemeier, R.W. and Isherwood, D. (1985) Radionuclide Migration Project 1984 Progress Report. Lawrence Livermore National Laboratory, UCRL-53628, Livermore, CA, $71 \mathrm{p}$.

Claassen, H.C. (1986) Late-Wisconsin paleohydrology of the west-central Amargosa Desert, Nevada, USA. Chemical Geology, v. 58, p. 311-323.

Department of Energy (2000). United States Nuclear Tests, July 1945 through September 1992, DOE/NV-209 (Rev. 15).

Fabryka-Martin, J., Wightman, S.J., Murphy, W.J., Wickham, M.P., Caffee, M.W., Nimz, G.J., Southon, J.R., and Sharma, P. (1993) Distribution of chlorine-36 in the unsaturated zone at Yucca Mt.: An indicator of fast transport paths. FOCUS '93: Site Characterization and Model Validation, Las Vegas, NV, 26-29 Sept 1993.

Garber, M.S. (1971) Hydraulic-test and quality -of-water data from hole U-3cn PS\#2, BILBY site, Nevada Test Site. U.S. Geological Survey Report USGS-474-102 (NTS230), Denver CO

Ingraham, N.L., Lyles, B.F., Jacobson, R.L., and Hess, J.W. (1991) Stable isotopic study of precipitation and spring discharge in southern Nevada. Journal of Hydrology, 125: $243-258$.

Kersting, A.B., Efurd, D.W., Finnegan, D.L., Rokop, D.J., Smith, D.K., and Thompson, J.L. (1999) Migration of plutonium in groundwater at the Nevada Test Site. Nature, 397: $56-59$

LLNL (1998) Isotopic Analyses: Monitoring Well ER-20-5. Letter report to Bob Bangerter, DOE/ERD, UGTA Operable Unit, December 3, 1998, 6 p.

LLNL (2004) Analytical Measurements: Standard Operating Procedures, Underground Test Area Project. Chemical Biology and Nuclear Science Division, Lawrence Livermore National Laboratory, 30 June 2004

Paces, J.B., Ludwig, K.R., Peterman, Z.E., and Neymark, L.A. (2002) ${ }^{234} U /{ }^{238} U$ evidence for local recharge and patterns of groundwater flow in the vicinity of Yucca Mountain, Nevada, USA. Applied Geochemistry, v. 17, p. 751-779

Peterman, Z.E., Spengler, R.W., Singer, F.R, and Dickerson, R.P. (1993) Isotopic and trace element variability in altered and unaltered tuffs at Yucca Mountain, Nevada. In: High Level Radioactive Waste Management, Proceedings of the Fourth Annual International Conference, Volume 2, 26-30 April 1993, Las Vegas, Nevada. American Nuclear Society, La Grange, Illinois, p. 1940-1947 
Rose, T.P., Benedict, F.C., Jr., Thomas, J.M., Sicke, W.S., Hershey, R.L., Paces, J.B., Farnham, I.M., and Peterman, Z.E. (2002) Geochemical data analysis and interpretation of the Pahute Mesa - Oasis Valley groundwater flow system, Nye County, Nevada. Lawrence Livermore National Laboratory draft report, prepared for the U.S. Department of Energy, Nevada Operations Office, Environmental Restoration Division, August 2002, $607 \mathrm{p}$

Rose, T.P., Eaton, G.F., and Kersting, A.B., eds. (2003) Hydrologic Resources Management Program and Underground Test Area Project FY 2001-2002 Progress Report. Lawrence Livermore National Laboratory Report, UCRL-ID-154357, 135 p

SNJV (2006) Geochemical and Isotopic Evaluation of Groundwater Movement in Corrective Action Unit 97: Yucca Flat/Climax Mine, Nevada Test Site, Nevada, S-N/99205-070, Las Vegas, NV

Thomas, J.M., Benedict, F.C., Jr., Rose, T.P., Hershey, R.L., Paces, J.B., Peterman, Z.E., Farnham, I.M., Johannesson, K.H., Singh, A.K., Stetzenbach, K.J., Hudson, G.B., Kenneally, J.M., Eaton, G.F., and Smith, D.K. (2002) Geochemical and isotopic interpretations of groundwater flow in the Oasis Valley flow system, southern Nevada. Desert Research Institute Publication No. 45190, August 2002, 202 p

Winograd, I.J., and Thordarson, W. (1975) Hydrogeologic and hydrochemical framework, south-central Great Basin, Nevada-California, with special reference to the Nevada Test Site. U.S. Geological Survey Professional Paper 712-C, 126 p. 
Table 1.1 Hotwell Site Information

\begin{tabular}{|c|c|c|c|c|c|c|c|c|c|c|c|c|}
\hline Well name & Test Name & Test Date & Latitude & Longitude & $\begin{array}{l}\text { Surface } \\
\text { Elevation }\end{array}$ & $\begin{array}{l}\text { Well } \\
\text { Depth }\end{array}$ & $\begin{array}{c}\text { Open } \\
\text { Interval }\end{array}$ & $\begin{array}{l}\text { Water } \\
\text { Depth }\end{array}$ & $\begin{array}{l}\text { Sample } \\
\text { Method }\end{array}$ & $\begin{array}{l}\text { Volume } \\
\text { Pumped }\end{array}$ & $\begin{array}{l}\text { Sample } \\
\text { Depth }\end{array}$ & $\begin{array}{c}\text { Sample } \\
\text { date }\end{array}$ \\
\hline Units & & & $(d m s)$ & $(d m s)$ & (ft) & (ft bgs) & (ft bgs) & (ft bgs) & & (gal) & (ft bgs) & \\
\hline \multicolumn{13}{|c|}{ Hot Wells - Frenchman Flat } \\
\hline UE5n & CAMBRIC & 14-May-65 & 364934 & 1160659 & 3112 & 1690 & $720-730$ & --- & pump & --- & --- & 12-Feb-04 \\
\hline UE5n & CAMBRIC & 14-May-65 & 364934 & 1160659 & 3112 & 1690 & $720-730$ & 702 & pump & --- & 702 & 19-Apr-01 \\
\hline UE5n & CAMBRIC & 14-May-65 & 364934 & 1160659 & 3112 & 1690 & $720-730$ & 705 & pump & --- & 730 & 09-Sep-99 \\
\hline RNM-1 & CAMBRIC & 14-May-65 & 364928 & 1155801 & 3135 & $1302^{*}$ & $1063-1075^{*}$ & $789^{*}$ & pump & $1.68 \mathrm{E}+04$ & --- & 03-Jun-04 \\
\hline RNM-1 & CAMBRIC & 14-May-65 & 364928 & 1155801 & 3135 & $1302^{*}$ & $1063-1075^{*}$ & $789^{*}$ & pump & --- & --- & 28-Jun-00 \\
\hline RNM-2S & CAMBRIC & 14-May-65 & 364921 & 1155801 & 3133 & 1120 & $1038-1119$ & 723 & pump & $6.39 \mathrm{E}+07$ & --- & 10-Jul-03 \\
\hline RNM-2S & CAMBRIC & 14-May-65 & 364921 & 1155801 & 3133 & 1120 & 1038-1119 & 723 & pump & $1.00 \mathrm{E}+07$ & --- & 09-May-03 \\
\hline RNM-2S & CAMBRIC & 14-May-65 & 364921 & 1155801 & 3133 & 1120 & $1038-1119$ & 723 & pump & -- & --- & 14-Jun-00 \\
\hline RNM-2S & CAMBRIC & 14-May-65 & 364921 & 1155801 & 3133 & 1120 & 1038-1119 & 723 & pump & --- & 800 & 11-Oct-99 \\
\hline \multicolumn{13}{|c|}{ Hot Wells - Yucca Flat } \\
\hline U4u PS2a & DALHART & 13-Oct-88 & 370513 & 1160251 & 4117 & 2280 & $1548-1644$ & 1636 & pump & $7.06 \mathrm{E}+03$ & --- & 09-Oct-03 \\
\hline U4u PS2a & DALHART & $13-O c t-88$ & 370513 & 1160251 & 4117 & 2280 & $1548-1644$ & 1636 & pump & -- & 1640 & 16-Aug-99 \\
\hline UE-7ns & BOURBON & 20-Jan-67 & 370556 & 1160009 & 4369 & 2205 & $1995-2199$ & 1969 & bailer & --- & 2025 & 21-Aug-01 \\
\hline UE-2ce & NASH & 19-Jan-67 & 370831 & 1160807 & 4764 & 1650 & $1385-1624$ & 1448 & bailer & --- & 1580 & 12-Jul-05 \\
\hline UE-2ce & NASH & 19-Jan-67 & 370831 & 1160807 & 4764 & 1650 & $1385-1624$ & 1470 & bailer & --- & 1550 & 22-Aug-01 \\
\hline U-3cn PS\#2 & BILBY & 13-Sep-63 & 370338 & 1160119 & 3994 & 2603 & $1680-1729$ & 1550 & pump & --- & $1652-1656$ & 09-Dec-04 \\
\hline U-3cn PS\#2 & BILBY & 13-Sep-63 & 370338 & 1160119 & 3994 & 2603 & $1680-1729$ & 1550 & pump & --- & $1652-1656$ & 18-Dec-01 \\
\hline \multicolumn{13}{|c|}{ Hot Wells - Pahute Mesa } \\
\hline U20n PS1 DDh & CHESHIRE & 14-Feb-76 & 371425 & 1162524 & 6468 & 4253 & $4100-4110$ & 2051 & pump & --- & 4100 & 09-Jul-03 \\
\hline U20n PS1 DDh & CHESHIRE & 14-Feb-76 & 371425 & 1162524 & 6468 & 4253 & $4100-4110$ & 2051 & pump & --- & 4100 & 12-Oct-99 \\
\hline U19ad PS1A & CHANCELLOR & 1-Sep-83 & 371613 & 1162117 & 6656 & $2609^{*}$ & $2407-2579^{*}$ & $2240^{*}$ & pump & $2.04 E+05$ & $2370^{*}$ & 27-Sep-04 \\
\hline U19q PS1d & CAMEMBERT & 26-Jun-75 & 371649 & 1162154 & 6740 & $4991^{*}$ & $3665-3678^{*}$ & $2185^{\star}$ & pump & $1.12 \mathrm{E}+04$ & $3000^{*}$ & 16-Jul-03 \\
\hline U19q PS1d & CAMEMBERT & 26-Jun-75 & 371649 & 1162154 & 6740 & $4991^{*}$ & $3665-3678^{*}$ & $2185^{\star}$ & pump & $1.47 \mathrm{E}+04$ & $3000^{*}$ & 21-Oct-98 \\
\hline U19v PS1ds & ALMENDRO & 06-Jun-73 & 371453 & 1162057 & 6842 & $3837^{*}$ & --- & & bailer & --- & & 23-Jul-03 \\
\hline U19v PS1ds & ALMENDRO & 06-Jun-73 & 371453 & 1162057 & 6842 & $3837^{*}$ & --- & $2187^{*}$ & bailer & --- & $3089^{*}$ & 31-May-01 \\
\hline U19v PS1ds & ALMENDRO & 06-Jun-73 & 371453 & 1162057 & 6842 & $3837^{\star}$ & --- & $2170^{*}$ & bailer & --- & $3089^{*}$ & 26-Sep-00 \\
\hline U19v PS1ds & ALMENDRO & 06-Jun-73 & 371453 & 1162057 & 6842 & $3837^{*}$ & --- & $2170^{*}$ & bailer & --- & $2999^{*}$ & 18-Aug-99 \\
\hline ER-20-5 \#1 & TYBO/BENHAM $†$ & 14-May-75 & 311312 & 1162838 & 6242 & 2823 & $2301-2573$ & 2055 & pump & --- & $2300-2572$ & 30-Nov-04 \\
\hline ER-20-5 \#1 & TYBO/BENHAM $†$ & 14-May-75 & 311312 & 1162838 & 6242 & 2823 & $2301-2573$ & 2055 & pump & --- & $2300-2572$ & 09-Jul-98 \\
\hline ER-20-5 \#3 & TYBO/BENHAM $†$ & 14-May-75 & 371311 & 1162838 & 6242 & 4294 & $3430-3882$ & 2060 & pump & --- & $3383-3405$ & 29-Nov-04 \\
\hline ER-20-5 \#3 & TYBO/BENHAM $\dagger$ & 14-Мay-75 & 371311 & 1162838 & 6242 & 4294 & $3430-3882$ & 2060 & pump & & $3383-340$ & 15-Nov-01 \\
\hline ER-20-5 \#3 & TYBO/BENHAM † & 14-May-75 & 371311 & 1162838 & 6242 & 4294 & $3430-3882$ & 2060 & pump & & $3383-340$ & 30-Apr-98 \\
\hline
\end{tabular}

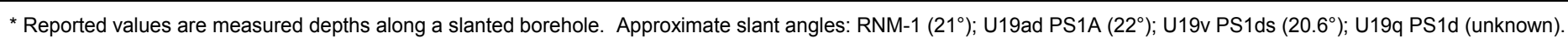

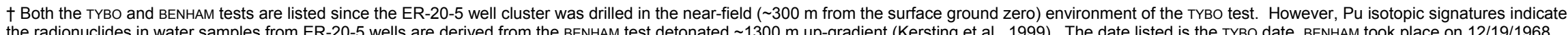


Table 1.2 Field parameter and anion data

\begin{tabular}{|c|c|c|c|c|c|c|c|c|c|c|c|c|}
\hline Well name & Test & Sample date & Volume Pumped & $\mathrm{pH}$ & $\mathbf{T}$ & Cond. & TDIC as $\mathrm{HCO}_{3}^{-}$ & $\mathbf{F}$ & $\mathrm{Cl}$ & $\mathrm{Br}$ & $\mathrm{NO}_{3}$ & $\mathrm{SO}_{4}$ \\
\hline Units & & date & (gal) & & $\left({ }^{\circ} \mathrm{C}\right)$ & $(\mu \mathrm{S} / \mathrm{cm})$ & $(m g / L)$ & $(m g / L)$ & $(\mathrm{mg} / \mathrm{L})$ & $(m g / L)$ & $(\mathrm{mg} / \mathrm{L})$ & $(\mathrm{mg} / \mathrm{L})$ \\
\hline \multicolumn{13}{|c|}{ Hot Wells - Frenchman Flat } \\
\hline UE5n & CAMBRIC & 12-Feb-04 & & $8.2^{*}$ & --- & $452^{*}$ & 178 & 0.7 & 11.7 & 0.1 & 6.0 & 31.9 \\
\hline UE5n & CAMBRIC & 19-Apr-01 & & 8.7 & 23.0 & 408 & 162 & 0.7 & 12.9 & $<0.1$ & 6.7 & 32.0 \\
\hline UE5n & CAMBRIC & 09-Sep-99 & & 8.4 & 26.5 & 453 & 183 & 0.8 & 12.0 & $<0.03$ & 8.1 & 31.8 \\
\hline RNM-1 & CAMBRIC & 03-Jun-04 & $1.68 \mathrm{E}+04$ & $7.8^{*}$ & --- & $432^{*}$ & 166 & 0.4 & 9.7 & 0.1 & 13.4 & 34.7 \\
\hline RNM-1 & CAMBRIC & 28-Jun-00 & & 8.0 & 26.0 & 416 & --- & 0.3 & 12.3 & 0.2 & 16.2 & 36.5 \\
\hline RNM-2S & CAMBRIC & 10-Jul-03 & $6.39 \mathrm{E}+07$ & 8.0 & 24.4 & 418 & 168 & 0.5 & 13.5 & 0.6 & 12.6 & 38.0 \\
\hline RNM-2S & CAMBRIC & 09-May-03 & $1.00 \mathrm{E}+07$ & 8.2 & 24.0 & 450 & 152 & 0.5 & 13.6 & 0.6 & 12.5 & 38.0 \\
\hline RNM-2S & CAMBRIC & 14-Jun-00 & -- & $7.8^{\star}$ & --- & $429^{*}$ & 130 & 0.4 & 14.8 & 0.2 & 14.3 & 36.8 \\
\hline RNM-2S & CAMBRIC & 11-Oct-99 & -- & 8.2 & 24.6 & 440 & 168 & 0.6 & 13.7 & $<0.03$ & 13.9 & 37.0 \\
\hline \multicolumn{13}{|c|}{ Hot Wells - Yucca Flat } \\
\hline U4u PS2a & DALHART & 09-Oct-03 & $7.06 \mathrm{E}+03$ & 6.7 & --- & 385 & 174 & 1.6 & 5.1 & $<0.03$ & 2.5 & 12.3 \\
\hline U4u PS2a & DALHART & 16-Aug-99 & --- & $8.2^{*}$ & --- & $352^{*}$ & 160 & 0.7 & 5.8 & $<0.03$ & 18.5 & 12.0 \\
\hline UE-7ns & BOURBON & 21-Aug-01 & wire-line bailer & $8.0^{*}$ & --- & $375^{\star}$ & 166 & 0.8 & 22.9 & $<0.1$ & $<0.09$ & 1.6 \\
\hline UE-2ce & NASH & 12-Jul-05 & wire-line bailer & 7.3 & --- & 466 & 337 & 1.1 & 14.2 & $<0.02$ & 1.6 & 16.6 \\
\hline UE-2ce & NASH & 22-Aug-01 & wire-line bailer & $7.9^{*}$ & --- & $435^{*}$ & 308 & 0.3 & 15.5 & $<0.1$ & $<0.09$ & 11.1 \\
\hline U-3cn PS\#2 & BILBY & 09-Dec-04 & $1.51 \mathrm{E}+04$ & $8.1^{*}$ & --- & 528 & 269 & 1.38 & 17.3 & 1.0 & 7.2 & 19.2 \\
\hline U-3cn PS\#2 & BILBY & 18-Dec-01 & --- & $8.1^{*}$ & --- & $490^{*}$ & 287 & 0.8 & 8.6 & 0.6 & 5.8 & 20.3 \\
\hline \multicolumn{13}{|c|}{ Hot Wells - Pahute Mesa } \\
\hline U20n PS1 DDh & CHESHIRE & 09-Jul-03 & --- & 8.5 & 38.4 & 330 & 90 & 3.6 & 10.9 & 0.6 & 1.9 & 27.9 \\
\hline U20n PS1 DDh & CHESHIRE & 12-Oct-99 & --- & 8.2 & 38.2 & 324 & 108 & 3.6 & 11.1 & $<0.03$ & 2.3 & 28.2 \\
\hline U19ad PS1A & CHANCELLOR & 27-Sep-04 & $2.04 \mathrm{E}+05$ & 9.4 & 47.0 & 941 & 110 & 36.0 & 43.5 & $<0.02$ & 3.0 & 106.4 \\
\hline U19q PS1d & CAMEMBERT & 16-Jul-03 & & & & & 496 & 15.5 & 7.2 & $<0.03$ & 0.5 & 20.8 \\
\hline U19q PS1d & CAMEMBERT & 21-Oct-98 & $1.47 \mathrm{E}+04$ & 7.2 & 33.0 & 959 & 1016 & 31.5 & 10.4 & $<0.1$ & $<0.07$ & 29.7 \\
\hline U19v PS1ds & ALMENDRO & 23-Jul-03 & --- & 9.3 & 35.7 & 517 & 207 & 8.9 & 53.2 & 0.1 & 0.7 & 0.8 \\
\hline U19v PS1ds & ALMENDRO & 31-May-01 & --- & --- & --- & --- & 250 & 9.7 & 66.5 & $<0.1$ & $<0.09$ & 2.5 \\
\hline U19v PS1ds & ALMENDRO & 26-Sep-00 & --- & $9.3^{*}$ & --- & $742^{*}$ & 262 & 9.5 & 48.2 & $<0.1$ & $<0.09$ & 3.8 \\
\hline U19v PS1ds & ALMENDRO & 18-Aug-99 & -- & $8.2^{*}$ & -- & $728^{*}$ & 120 & 9.9 & 40.5 & $<0.03$ & $<0.02$ & 3.9 \\
\hline ER-20-5 \#1 & TYBO/BENHAM $†$ & 30-Nov-04 & --- & $8.1^{*}$ & --- & $545^{*}$ & 193 & 10.8 & 24.7 & 0.1 & 1.8 & 43.2 \\
\hline ER-20-5 \#1 & TYBO/BENHAM † & 09-Jul-98 & --- & 8.1 & 32.3 & 510 & 182 & 9.6 & 24.5 & $<0.04$ & 1.4 & 40.4 \\
\hline ER-20-5 \#3 & TYBO/BENHAM $†$ & 29-Nov-04 & --- & $8.2^{*}$ & -- & $376^{*}$ & 135 & 4.1 & 17.4 & 0.1 & 1.7 & 35.3 \\
\hline ER-20-5 \#3 & TYBO/BENHAM $\dagger$ & 15-Nov-01 & --- & $8.0^{*}$ & -- & $345^{*}$ & 110 & 3.6 & 18.9 & 0.8 & 2.6 & 35.3 \\
\hline ER-20-5 \#3 & TYBO/BENHAM $\dagger$ & 30-Apr-98 & --- & 8.2 & 35.3 & 335 & 107 & 3.2 & 17.3 & $<0.02$ & 1.2 & 33.3 \\
\hline
\end{tabular}

${ }^{*} \mathrm{pH}$ and conductivity values marked with an asterisk are laboratory measurements

† Both the TYBO and BENHAM tests are listed since the ER-20-5 well cluster was drilled in the near-field ( $\sim 300 \mathrm{~m}$ from the surface ground zero) environment of the TYBO test. However, Pu isotopic signatures indicate the radionuclides in water samples from ER-20-5 wells are derived from the BENHAM test detonated 1300 m up-gradient (Kersting et al., 1999). 
Table 1.3 Cations and metals data

\begin{tabular}{|c|c|c|c|c|c|c|c|c|c|c|c|c|c|c|c|c|c|c|c|c|c|}
\hline Well name & Test & $\begin{array}{c}\text { Sample } \\
\text { date }\end{array}$ & $\mathrm{Na}$ & $\mathbf{K}$ & Ca & Mg & Li & Al & Si & $\mathrm{Fe}$ & Mn & As & Se & $\mathrm{Sr}$ & Mo & Sb & I & $\mathrm{Ba}$ & $\mathrm{Pb}$ & $\mathbf{U}$ & $\mathrm{Pu}$ \\
\hline Unit & & & $(\mathrm{mg} / \mathrm{L})$ & $(\mathrm{mg} / \mathrm{L})$ & $(\mathrm{mg} / \mathrm{L})$ & $(\mathrm{mg} / \mathrm{L})$ & $(\mathrm{mg} / \mathrm{L})$ & $(\mathrm{mg} / \mathrm{L})$ & $(\mathrm{mg} / \mathrm{L})$ & $(\mathrm{mg} / \mathrm{L})$ & $(\mu \mathrm{g} / \mathrm{L})$ & $(\mu \mathrm{g} / \mathrm{L})$ & $(\mu g / L)$ & $(\mu g / L)$ & $(\mu \mathrm{g} / \mathrm{L})$ & $(\mu g / L)$ & $(\mu g / L)$ & $(\mu g / L)$ & $(\mu g / L)$ & $(\mu \mathrm{g} / \mathrm{L})$ & $(p g / L)$ \\
\hline \multicolumn{22}{|c|}{ Hot Wells - Frenchman Flat } \\
\hline UE5n & CAMBRIC & 12-Feb-04 & 86.7 & 7.7 & 8.3 & 2.2 & $<0.01$ & 0.021 & 41.3 & 1.08 & 44 & 11.9 & 1.5 & 28 & 6.1 & 0.39 & 19.0 & 4.7 & 2.8 & 4.45 & $<0.2$ \\
\hline UE5n & CAMBRIC & 19-Apr-01 & 76.0 & 8.0 & 8.6 & 2.0 & 0.02 & $<0.05$ & 27.4 & $<0.04$ & $<6$ & 22 & --- & 22 & 4 & --- & 20.3 & 21 & --- & 0.41 & -- \\
\hline UE5n & CAMBRIC & 09-Sep-99 & 86.0 & 8.0 & 7.5 & 2.0 & $<0.05$ & $<0.02$ & --- & 0.06 & 10 & 9 & $<2$ & 50 & 5 & --- & 30.0 & $<5$ & 0.37 & 4.00 & --- \\
\hline RNM-1 & CAMBRIC & 03-Jun-04 & 45.5 & 7.5 & 26.8 & 10.4 & 0.03 & 0.005 & 50.5 & 0.003 & 9.9 & 9.7 & 1.6 & 166 & 4.4 & 0.18 & 16.2 & 19.3 & 3.4 & 3.84 & 0.17 \\
\hline RNM-1 & CAMBRIC & 28-Jun-00 & 44.0 & 8.0 & 26.0 & 9.4 & 0.03 & 0.4 & 20 & 0.18 & $<6$ & 9.8 & 1.5 & 300 & 5.1 & 0.15 & 17.9 & 18.5 & 0.01 & 4.00 & -- \\
\hline RNM-2S & CAMBRIC & 10-Jul-03 & 56.7 & 7.8 & 15.1 & 5.7 & 0.02 & $<0.05$ & 37.2 & $<0.04$ & $<6$ & $<20$ & $<24$ & 102 & $<3$ & --- & 12.2 & 6 & $<14$ & 4.91 & $<0.2$ \\
\hline RNM-2S & CAMBRIC & 09-Мау-03 & 57.2 & 7.9 & 16.3 & 5.9 & 0.02 & $<0.05$ & 35.5 & $<0.04$ & $<6$ & 27 & $<24$ & 101 & $<3$ & --- & 12.1 & 5 & $<14$ & 4.93 & $<0.2$ \\
\hline RNM-2S & CAMBRIC & 14-Jun-00 & 62.0 & 9.7 & 18.0 & 5.2 & 0.02 & 0.4 & 35 & 0.18 & $<6$ & 10.5 & 1.6 & 140 & 4.4 & 0.14 & 12.0 & 17.3 & $<0.01$ & 5.00 & --- \\
\hline RNM-2S & CAMBRIC & 11-Oct-99 & 63.0 & 9.2 & 17.0 & 5.6 & $<0.05$ & $<0.02$ & 32 & 0.01 & $<2$ & 5 & $<2$ & 110 & 3 & --- & 11.0 & $<5$ & 0.24 & 4.00 & --- \\
\hline \multicolumn{22}{|c|}{ Hot Wells - Yucca Flat } \\
\hline U4u PS2a & DALHART & 09-Oct-03 & 87.0 & 22.1 & 12.7 & 1.2 & 0.02 & 10.7 & 61.6 & 1.99 & 125 & 13.9 & 0.9 & 25 & 5.1 & 0.54 & 7.1 & 27.3 & 17.6 & 7.43 & 5.0 \\
\hline U4u PS2a & DALHART & 16-Aug-99 & 72.0 & 14.0 & 13.1 & 2.7 & 0.14 & 12.0 & 37 & 1.30 & 90 & 4 & $<1$ & 30 & $<1$ & --- & 3.1 & 30 & 8.6 & 4.20 & 8.7 \\
\hline UE-7ns & BOURBON & 21-Aug-01 & 67.4 & 4.9 & 20.9 & 3.6 & 0.06 & $<0.05$ & 10.0 & 0.21 & 55 & 21 & $<24$ & 70 & 26 & -- & 19.2 & 77 & $<14$ & 0.04 & $<0.6$ \\
\hline UE-2ce & NASH & 12-Jul-05 & 38.1 & 17.6 & 46.0 & 24.9 & 0.08 & 0.0 & 13 & 12 & 199.6 & 5.5 & $<0.6$ & 194 & 8.9 & 1.09 & 4.0 & 22.5 & 551 & 2.00 & $<1$ \\
\hline UE-2ce & NASH & 22-Aug-01 & 45.6 & 21.8 & 49.1 & 25.4 & 0.10 & $<0.05$ & 22.2 & 0.11 & 66 & $<20$ & $<24$ & 160 & 17 & --- & 7.4 & 47 & $<14$ & 0.39 & $<0.6$ \\
\hline U-3cn PS\#2 & BILBY & 09-Dec-04 & 89.4 & 15.3 & 11.9 & 3.3 & 0.02 & 1.23 & 46.9 & 4.23 & 173 & 5.6 & 1.5 & 21 & 7.2 & 0.88 & 9.5 & 18.7 & 104 & 10.31 & -- \\
\hline U-3cn PS\#2 & BILBY & 18-Dec-01 & 98.4 & 18.3 & 14.4 & 3.3 & 0.05 & 0.3 & 28.7 & 0.30 & 78 & $<20$ & $<24$ & 24 & 3.5 & --- & 9.1 & 4 & $<14$ & 10.67 & $<0.6$ \\
\hline \multicolumn{22}{|c|}{ Hot Wells - Pahute Mesa } \\
\hline U20n PS1 DDh & CHESHIRE & 09-Jul-03 & 60.7 & 1.9 & 3.8 & 0.1 & 0.06 & 0.7 & 30.9 & 0.81 & 170 & $<20$ & $<24$ & 12 & 9.1 & --- & 2.0 & 3 & $<14$ & 2.07 & 4.1 \\
\hline U20n PS1 DDh & CHESHIRE & 12-Oct-99 & 65.0 & 2.2 & 4.7 & 0.1 & $<0.05$ & 0.1 & 24 & 1.4 & 110 & 3 & $<2$ & 10 & 10 & -- & 13.0 & $<5$ & 0.67 & 2.30 & 7.0 \\
\hline U19ad PS1A & CHANCELLOR & 27-Sep-04 & 156.2 & 6.6 & 0.8 & 0.0 & 0.73 & 9.69 & 115.3 & 2.02 & 357 & 232 & 2.4 & 17 & 1024 & 30.6 & 19.4 & 72.9 & 21.8 & 2.60 & 369.9 \\
\hline U19q PS1d & CAMEMBERT & 16-Jul-03 & 199.0 & 9.0 & 5.0 & 0.2 & 0.49 & 50 & 20.2 & 11.53 & 586 & 14.6 & 0.3 & 7.9 & 40.7 & 0.89 & 4.0 & 9.7 & 16.4 & 2.61 & $<0.2$ \\
\hline U19q PS1d & CAMEMBERT & 21-Oct-98 & 342.0 & 10.3 & 3.2 & 0.0 & 1.06 & 70 & 32 & 5.77 & 530 & 4 & $<3$ & $<0.01$ & 40 & --- & 10.0 & $<10$ & 0.60 & $<0.5$ & --- \\
\hline U19v PS1ds & ALMENDRO & 23-Jul-03 & 140.0 & 8.8 & 1.0 & 0.1 & 0.17 & 0.04 & 22.8 & 10.6 & 160 & 341 & 0.08 & 18 & 2059 & 250 & 10.1 & 60 & 82 & 0.11 & $<0.2$ \\
\hline U19v PS1ds & ALMENDRO & 31-May-01 & 173.0 & 14.0 & 1.5 & 0.2 & 0.28 & 0.3 & 2.0 & 3.7 & 330 & 140 & $<24$ & 44 & 1200 & --- & 13.7 & 60 & 250 & 0.03 & 2.1 \\
\hline U19v PS1ds & ALMENDRO & 26-Sep-00 & 131.0 & 11.6 & 0.7 & 0.1 & 0.24 & 0.3 & 6.6 & 0.19 & --- & 50 & $<24$ & --- & 1100 & --- & 11.4 & 20 & 22 & 0.02 & --- \\
\hline U19v PS1ds & ALMENDRO & 18-Aug-99 & 162.0 & 9.8 & 0.9 & 0.2 & 0.42 & 0.5 & 11 & 12.0 & 250 & 1716 & 8 & 40 & 1226 & --- & 7.4 & 70 & 63 & $<0.5$ & 58.2 \\
\hline ER-20-5 \#1 & TYBO/BENHAM $†$ & 30-Nov-04 & 117.5 & 4.6 & 6.2 & 0.1 & $<0.01$ & 6.53 & 67.9 & 0.40 & 22 & 10.3 & 0.9 & 21 & 40.2 & 1.30 & 5.4 & 13.3 & 9.1 & 14.09 & 6.4 \\
\hline ER-20-5 \#1 & TYBO/BENHAM † & 09-Jul-98 & 106.0 & 5.7 & 7.2 & 0.4 & 0.09 & 5.76 & 48.4 & 1.32 & 50 & 3.1 & $<3$ & 20 & 0.03 & --- & 7.0 & $<10$ & 2.9 & 15.00 & -- \\
\hline ER-20-5 \#3 & TYBO/BENHAM $\dagger$ & 29-Nov-04 & 80.4 & 3.5 & 3.5 & $<0.04$ & $<0.01$ & 8.53 & 54.6 & 2.24 & 129 & 14.4 & 0.9 & 30 & 10.4 & 0.46 & 4.7 & 28.8 & 18.2 & 5.64 & 0.6 \\
\hline ER-20-5 \#3 & TYBO/BENHAM $\dagger$ & 15-Nov-01 & 87.1 & 3.3 & 4.4 & 0.1 & 0.07 & 3.60 & 29 & 1.9 & 75 & 23 & $<24$ & 31 & 5.8 & --- & 5.8 & 17 & $<14$ & 12.80 & $<0.6$ \\
\hline ER-20-5 \#3 & TYBO/BENHAM $\dagger$ & 30-Apr-98 & 68.0 & 2.1 & 1.8 & 0.1 & 0.07 & 1.03 & 24 & 0.46 & 20 & 4.8 & $<3$ & 7 & $<0.01$ & --- & 8.0 & $<10$ & 0.9 & 2.70 & --- \\
\hline
\end{tabular}

† Both the TYBO and BENHAM tests are listed since the ER-20-5 well cluster was drilled in the near-field ( $\sim 300 \mathrm{~m}$ from the surface ground zero) environment of the TYBO test. However, Pu isotopic signatures indicate the radionuclides in water samples from ER-20-5 wells are derived from the BENHAM test detonated $~ 1300 \mathrm{~m}$ up-gradient (Kersting et al., 1999). 
Table 1.4 Trace metal data

\begin{tabular}{|c|c|c|c|c|c|c|c|c|c|c|c|c|c|c|c|c|}
\hline Well name & Test & Sample date & V & $\mathrm{Cr}$ & Co & $\mathrm{Ni}$ & $\mathrm{Cu}$ & $Z n$ & $\mathrm{Br} *$ & $\mathbf{R b}$ & $\mathbf{N b}$ & $\mathbf{R u}$ & Cd & Sn & Cs & Eu \\
\hline Unit & & & $(\mu g / L)$ & $(\mu g / L)$ & $(\mu g / L)$ & $(\mu g / L)$ & $(\mu g / L)$ & $(\mu g / L)$ & $(\mu g / L)$ & $(\mu g / L)$ & $(\mu g / L)$ & $(\mu g / L)$ & $(\mu g / L)$ & $(\mu g / L)$ & $(\mu g / L)$ & $(\mu g / L)$ \\
\hline \multicolumn{17}{|c|}{ Hot Wells - Frenchman Flat } \\
\hline UE5n & CAMBRIC & 12-Feb-04 & 15.5 & 1.29 & 0.17 & 0.65 & BD & 37.0 & 129 & 8.4 & 0.22 & 0.078 & 0.09 & 0.83 & 0.09 & 0.05 \\
\hline UE5n & CAMBRIC & 19-Apr-01 & & & & & & & & & & & & & & \\
\hline UE5n & CAMBRIC & 09-Sep-99 & & & & & & & & & & & & & & \\
\hline RNM-1 & CAMBRIC & 03-Jun-04 & 12.6 & 1.89 & 0.08 & 3.24 & $\mathrm{BD}$ & 21.5 & 119 & 8.1 & 0.08 & 0.019 & 0.04 & 1.05 & 0.05 & 0.02 \\
\hline RNM-1 & CAMBRIC & 28-Jun-00 & 12.8 & 2.15 & 0.05 & 1.95 & BD & $\mathrm{BD}$ & 123 & 8.3 & 0.15 & 0.007 & 0.01 & 0.06 & 0.02 & $\mathrm{BD}$ \\
\hline RNM-2S & CAMBRIC & 10-Jul-03 & & & & & & & & & & & & & & \\
\hline RNM-2S & CAMBRIC & 09-May-03 & & & & & & & & & & & & & & \\
\hline RNM-2S & CAMBRIC & 14-Jun-00 & 15.8 & 3.58 & 0.08 & 0.75 & $\mathrm{BD}$ & $\mathrm{BD}$ & 139 & 10 & 0.10 & 0.003 & 0.01 & 0.02 & 0.03 & $\mathrm{BD}$ \\
\hline RNM-2S & CAMBRIC & 11-Oct-99 & & & & & & & & & & & & & & \\
\hline \multicolumn{17}{|c|}{ Hot Wells - Yucca Flat } \\
\hline U4u PS2a & DALHART & 09-Oct-03 & 7.0 & 3.02 & 0.18 & 0.99 & $\mathrm{BD}$ & 38.8 & 50 & 46 & 4.25 & 0.004 & 0.07 & 0.20 & 1.47 & 0.05 \\
\hline U4u PS2a & DALHART & 16-Aug-99 & & & & & & & & & & & & & & \\
\hline UE-7ns & BOURBON & 21-Aug-01 & & & & & & & & & & & & & & \\
\hline UE-2ce & $\mathrm{NASH}$ & 12-Jul-05 & $<0.9$ & 3.4 & 1.43 & 9 & 38.8 & 488 & $\mathrm{n} / \mathrm{a}$ & 39.7 & $<0.09$ & $<0.42$ & $<0.6$ & $\mathrm{n} / \mathrm{a}$ & $<0.6$ & $<0.012$ \\
\hline UE-2ce & NASH & 22-Aug-01 & & & & & & & & & & & & & & \\
\hline U-3cn PS\#2 & BILBY & 09-Dec-04 & 5.8 & 1.55 & 0.43 & 11.6 & $\mathrm{BD}$ & 126 & 68 & 51 & 0.15 & $\mathrm{BD}$ & 0.92 & 0.06 & 0.40 & $\mathrm{BD}$ \\
\hline U-3cn PS\#2 & BILBY & 18-Dec-01 & & & & & & & & & & & & & & \\
\hline \multicolumn{17}{|c|}{ Hot Wells - Pahute Mesa } \\
\hline U20n PS1 DDh & CHESHIRE & 09-Jul-03 & & & & & & & & & & & & & & \\
\hline U20n PS1 DDh & CHESHIRE & 12-Oct-99 & & & & & & & & & & & & & & \\
\hline U19ad PS1A & CHANCELLOR & 27-Sep-04 & 101 & 0.44 & 0.19 & 1.42 & $\mathrm{BD}$ & 42.3 & 158 & 80 & 9.10 & 0.003 & 0.58 & 0.69 & 11.7 & 0.44 \\
\hline U19q PS1d & CAMEMBERT & 16-Jul-03 & 1.5 & 3.86 & 0.66 & 50.1 & $\mathrm{BD}$ & 336 & 56 & 57 & 3.95 & 0.002 & 0.12 & 1.61 & 3.69 & 0.01 \\
\hline U19q PS1d & CAMEMBERT & 21-Oct-98 & & & & & & & & & & & & & & \\
\hline U19v PS1ds & ALMENDRO & 23-Jul-03 & 1.4 & 0.09 & 0.37 & 1.63 & $\mathrm{BD}$ & 65.6 & 127 & 66 & 0.25 & 0.005 & 0.78 & 0.02 & 1.24 & $\mathrm{BD}$ \\
\hline U19v PS1ds & ALMENDRO & 31-May-01 & & & & & & & & & & & & & & \\
\hline U19v PS1ds & ALMENDRO & 26-Sep-00 & & & & & & & & & & & & & & \\
\hline U19v PS1ds & ALMENDRO & 18-Aug-99 & & & & & & & & & & & & & & \\
\hline ER-20-5 \#1 & TYBO/BENHAM $†$ & 30-Nov-04 & 3.2 & 0.98 & 0.11 & 61.5 & $\mathrm{BD}$ & 42.0 & 134 & 27 & 1.24 & 0.002 & 0.05 & 0.16 & 2.86 & 0.004 \\
\hline ER-20-5 \#1 & TYBO/BENHAM $\dagger$ & 09-Jul-98 & & & & $<0.01$ & & & & & & & & & & \\
\hline ER-20-5 \#3 & TYBO/BENHAM $\dagger$ & 29-Nov-04 & 6.1 & 1.94 & 0.38 & 68.9 & $\mathrm{BD}$ & 54.4 & 102 & 38 & 4.89 & 0.001 & 0.20 & 0.56 & 2.74 & 0.03 \\
\hline ER-20-5 \#3 & TYBO/BENHAM $\dagger$ & 15-Nov-01 & & & & & & & & & & & & & & \\
\hline ER-20-5 \#3 & TYBO/BENHAM $†$ & 30-Apr-98 & & & & $<0.01$ & & & & & & & & & & \\
\hline
\end{tabular}

† Both the TYBO and BENHAM tests are listed since the ER-20-5 well cluster was drilled in the near-field ( $\sim 300 \mathrm{~m}$ from the surface ground zero) environment of the TYBO test. However, Pu isotopic signatures indicate the radionuclides in water samples from ER-20-5 wells are derived from the BENHAM test detonated $\sim 1300 \mathrm{~m}$ up-gradient (Kersting et al., 1999). 
Table 1.5 Stable Isotope data

\begin{tabular}{|c|c|c|c|c|c|c|c|c|c|c|c|c|c|c|}
\hline Well name & Test & Sample date & $\delta D_{\text {sMow }}$ & $\delta^{18} \mathrm{O}_{\text {sMow }}$ & $\delta^{13} C_{P D B}$ & ${ }^{3} \mathrm{He}$ & ${ }^{4} \mathrm{He}$ & $\mathrm{R}_{\mathrm{R}} \mathrm{R}$ & ${ }^{20} \mathrm{Ne}^{*}$ & ${ }^{40} \mathrm{Ar}^{*}$ & $\mathrm{Kr}$ & ${ }^{130} \mathrm{Xe}^{*}$ & ${ }^{87} \mathrm{Sr} l^{86} \mathrm{Sr}$ & $\delta^{87} \mathrm{Sr}$ \\
\hline Unit & & & $\%$ & $\%$ & $\%$ & atoms $/ g$ & atoms $/ g$ & $\begin{array}{c}{ }^{3} \mathrm{He} /{ }^{4} \mathrm{He} \\
\text { sample/air }\end{array}$ & atoms $/ g$ & atoms $/ g$ & atoms $/ g$ & atoms $/ g$ & ratio & $\%$ \\
\hline \multicolumn{15}{|c|}{ Hot Wells - Frenchman Flat } \\
\hline UE $5 n$ & CAMBRIC & 12-Feb-04 & -105 & -13.5 & -8.0 & -.- & --- & --- & --- & --- & --- & --. & 0.70871 & -0.69 \\
\hline UE5n & CAMBRIC & 19-Apr-01 & -105 & -13.4 & -6.7 & $2.70 \mathrm{E}+09$ & $2.34 \mathrm{E}+12$ & $8.36 \mathrm{E}+02$ & $4.66 \mathrm{E}+12$ & $8.30 \mathrm{E}+15$ & --- & $1.14 \mathrm{E}+10^{*}$ & 0.71039 & 1.68 \\
\hline UE5n & CAMBRIC & 09-Sep-99 & -106 & -13.4 & -8.3 & $1.95 \mathrm{E}+09$ & $3.16 \mathrm{E}+12$ & $4.48 \mathrm{E}+02$ & $7.25 E+12$ & $1.04 \mathrm{E}+16$ & -- & 1.19E+10* & --- & --- \\
\hline RNM-1 & CAMBRIC & 03-Jun-04 & -104 & -12.8 & -8.5 & --- & --- & --- & --- & --- & --- & --- & 0.70772 & -2.09 \\
\hline RNM-1 & CAMBRIC & 28-Jun-00 & -104 & -12.7 & --- & $1.43 \mathrm{E}+09$ & $2.05 \mathrm{E}+12$ & $5.05 \mathrm{E}+02$ & $4.15 \mathrm{E}+12$ & $7.63 \mathrm{E}+15$ & --- & --- & --- & --- \\
\hline RNM-2S & CAMBRIC & 10-Jul-03 & -105 & -13.1 & -9.5 & $4.29 \mathrm{E}+10$ & $1.94 E+13$ & $1.60 \mathrm{E}+03$ & $7.17 \mathrm{E}+13$ & --- & --- & $3.63 E+10$ & 0.71049 & 1.82 \\
\hline RNM-2S & CAMBRIC & 09-May-03 & -105 & -13.1 & -8.2 & $2.53 E+10$ & $2.77 \mathrm{E}+12$ & $6.62 E+03$ & $3.34 \mathrm{E}+12$ & --- & --- & $1.35 E+10$ & 0.71051 & 1.85 \\
\hline RNM-2S & CAMBRIC & 14-Jun-00 & -105 & -13.0 & -5.3 & $2.50 \mathrm{E}+10$ & $6.43 E+12$ & $2.84 \mathrm{E}+03$ & $5.50 \mathrm{E}+12$ & $9.11 \mathrm{E}+15$ & --- & --- & --- & --- \\
\hline RNM-2S & CAMBRIC & 11-Oct-99 & -104 & -12.9 & -9.3 & $2.35 \mathrm{E}+10$ & $6.40 \mathrm{E}+12$ & $2.65 \mathrm{E}+03$ & $4.35 \mathrm{E}+12$ & $8.09 \mathrm{E}+15$ & --- & -- & -- & --- \\
\hline \multicolumn{15}{|c|}{ Hot Wells - Yucca Flat } \\
\hline U4u PS2a & DALHART & 09-Oct-03 & -104.5 & -13.5 & -9.4 & $5.56 \mathrm{E}+11$ & $5.57 \mathrm{E}+12$ & $7.23 \mathrm{E}+04$ & $1.33 \mathrm{E}+13$ & --- & --- & $1.08 \mathrm{E}+10$ & 0.71275 & 5.01 \\
\hline U4u PS2a & DALHART & 16-Aug-99 & -100 & -12.8 & -8.7 & --- & --- & --- & --- & --- & --- & -- & -- & --- \\
\hline UE-7ns & BOURBON & 21-Aug-01 & -106 & -14.0 & -2.0 & $1.93 \mathrm{E}+10$ & $2.45 \mathrm{E}+12$ & $5.71 \mathrm{E}+03$ & $6.52 \mathrm{E}+12$ & $9.30 \mathrm{E}+15$ & --- & $1.10 \mathrm{E}+10^{*}$ & --- & --- \\
\hline UE-2ce & NASH & 12-Jul-05 & -98 & -12.6 & -6.6 & --- & --- & --- & --- & --- & --- & --- & 0.71137 & 3.06 \\
\hline UE-2ce & NASH & 22-Aug-01 & -100 & -12.9 & -5.3 & $4.78 \mathrm{E}+09$ & $2.36 \mathrm{E}+12$ & $1.47 \mathrm{E}+03$ & $7.14 \mathrm{E}+12$ & $9.31 \mathrm{E}+15$ & --- & $1.09 \mathrm{E}+10^{*}$ & --- & --- \\
\hline U-3cn PS\#2 & BILBY & 09-Dec-04 & -110 & -13.9 & -7.0 & --- & --- & --- & --- & --- & --- & --- & 0.70984 & 0.9 \\
\hline U-3cn PS\#2 & BILBY & 18-Dec-01 & -108 & -13.9 & -3.8 & $5.98 \mathrm{E}+11$ & $3.59 \mathrm{E}+12$ & $1.21 \mathrm{E}+05$ & $7.41 \mathrm{E}+12$ & $9.30 \mathrm{E}+15$ & --- & $1.00 \mathrm{E}+10^{*}$ & 0.70974 & 0.76 \\
\hline \multicolumn{15}{|c|}{ Hot Wells - Pahute Mesa } \\
\hline U20n PS1 DDh & CHESHIRE & 09-Jul-03 & -114 & -15.0 & -4.0 & $2.12 \mathrm{E}+12$ & $1.02 E+13$ & $1.51 \mathrm{E}+05$ & $5.63 \mathrm{E}+12$ & --- & --- & $1.24 \mathrm{E}+10$ & 0.71088 & 2.37 \\
\hline U20n PS1 DDh & CHESHIRE & 12-Oct-99 & -113 & -15.0 & -6.0 & $2.01 \mathrm{E}+12$ & $1.01 \mathrm{E}+13$ & $1.44 \mathrm{E}+05$ & $5.70 \mathrm{E}+12$ & $6.45 \mathrm{E}+15$ & --- & --- & 0.71078 & 2.23 \\
\hline U19ad PS1a & CHANCELLOR & 27-Sep-04 & -112 & -14.7 & -8.6 & --- & --- & --- & --- & --- & --- & --- & 0.71049 & 1.82 \\
\hline U19q PS1d & CAMEMBERT & 16-Jul-03 & -114 & -15.0 & +0.5 & --- & --- & --- & --- & -- & & --- & 0.71190 & 3.81 \\
\hline U19q PS1d & CAMEMBERT & 21-Oct-98 & -113 & -14.6 & +1.5 & $2.02 E+12$ & $1.85 \mathrm{E}+14$ & $7.90 \mathrm{E}+03$ & $7.73 E+12$ & --- & --- & --- & 0.71260 & 4.79 \\
\hline U19v PS1ds & ALMENDRO & 23-Jul-03 & -112 & -13.4 & +35.4 & $7.64 \mathrm{E}+10$ & $9.52 \mathrm{E}+11$ & $5.82 \mathrm{E}+04$ & $5.74 \mathrm{E}+12$ & --- & --- & $1.69 \mathrm{E}+10$ & 0.71113 & 2.72 \\
\hline U19v PS1ds & ALMENDRO & 31-May-01 & -112 & -13.3 & +30.1 & $3.75 \mathrm{E}+11$ & $1.50 \mathrm{E}+12$ & $1.81 \mathrm{E}+05$ & $4.42 E+12$ & $1.45 \mathrm{E}+16$ & & $3.86 \mathrm{E}+10^{*}$ & --- & --- \\
\hline U19v PS1ds & ALMENDRO & 26-Sep-00 & -111 & -13.4 & +30.2 & --- & --- & --- & --- & --- & --- & --- & --- & --- \\
\hline U19v PS1ds & ALMENDRO & 18-Aug-99 & -111 & -13.4 & +45.0 & --- & --- & --- & --- & --- & --- & --- & --- & --- \\
\hline ER-20-5 \#1 & TYBO/BENHAM & 30-Nov-04 & -115 & -14.9 & -4.7 & --- & --- & --- & --- & --- & --- & --- & 0.71047 & 7.79 \\
\hline ER-20-5 \#1 & TYBO/BENHAM $\dagger$ & 09-Jul-98 & & & -2.48 & $4.15 \mathrm{E}+12$ & $2.64 \mathrm{E}+13$ & $1.14 \mathrm{E}+05$ & $7.55 \mathrm{E}+12$ & --- & --- & -- & --- & 2.59 \\
\hline ER-20-5 \#3 & TYBO/BENHAM $†$ & 29-Nov-04 & -114 & -15.1 & -9.3 & --- & --- & --- & --- & --- & --- & --- & 0.70841 & -1.11 \\
\hline ER-20-5 \#3 & TYBO/BENHAM $\dagger$ & 15-Nov-01 & -114 & -15.0 & -4.0 & $8.09 E+09$ & $7.48 \mathrm{E}+12$ & $7.84 \mathrm{E}+02$ & $7.49 \mathrm{E}+12$ & --- & --- & $1.13 \mathrm{E}+10^{*}$ & 0.70864 & -0.79 \\
\hline ER-20-5 \#3 & TYBO/BENHAM $†$ & 30-Apr-98 & & & -5.6 & $1.21 \mathrm{E}+10$ & $1.21 \mathrm{E}+13$ & $7.23 \mathrm{E}+02$ & $7.23 E+12$ & --- & --- & --- & --- & -0.73 \\
\hline
\end{tabular}

* Inconsistencies have been identified in the noble gas data. This issue will be addressed in the FY06 HRMP report.

† Both the TYBO and BENHAM tests are listed since the ER-20-5 well cluster was drilled in the near-field ( $300 \mathrm{~m}$ from the surface ground zero) environment of the TYBO test.

However, Pu isotopic signatures indicate the radionuclides in water samples from ER-20-5 wells are derived from the BENHAM test detonated $\sim 1300 \mathrm{~m}$ up-gradient (Kersting et al., 1999). 
Table 1.6 Radiochemical data

\begin{tabular}{|c|c|c|c|c|c|c|c|c|c|c|c|c|}
\hline Well name & Test & Sample date & ${ }^{3} \mathrm{H}$ & ${ }^{3} \mathrm{H}$ & ${ }^{14} \mathrm{C}$ & ${ }^{14} \mathrm{C}$ & ${ }^{36} \mathrm{Cl} / \mathrm{Cl}$ & ${ }^{36} \mathrm{Cl}$ & ${ }^{85} \mathrm{Kr}$ & ${ }^{99} \mathrm{Tc}$ & ${ }^{129}\left|/^{127}\right|$ & ${ }^{129} \mathbf{I}$ \\
\hline Unit & & date & $(p C i / L)$ & $(p C i / L)$ & $(p m c)$ & $(p C i / L)$ & ratio & $(p C i / L)$ & $(p C i / L)$ & $(p C i / L)$ & ratio & $(p C i / L)$ \\
\hline Half-life (a) & & collected & 12.32 & 12.32 & 5730 & 5730 & & $3.01 E+05$ & 10.73 & $2.13 E+05$ & & $1.57 E+07$ \\
\hline Ref. date & & in field & collect. & time zero & collect. & collect. & & collect. & collect. & collect. & & collect. \\
\hline \multicolumn{13}{|c|}{ Hot Wells - Frenchman Flat } \\
\hline UE5n & CAMBRIC & 12-Feb-04 & $1.5 \mathrm{E}+05$ & $1.3 \mathrm{E}+06$ & $1.66 \mathrm{E}+01$ & $3.55 \mathrm{E}-02$ & $6.49 \mathrm{E}-10$ & $2.51 \mathrm{E}-01$ & --- & $5.6 \mathrm{E}-03$ & $5.02 \mathrm{E}-07$ & $1.70 \mathrm{E}-03$ \\
\hline UE5n & CAMBRIC & 19-Apr-01 & $1.4 \mathrm{E}+05$ & $1.1 \mathrm{E}+06$ & $2.84 \mathrm{E}+01$ & $5.53 \mathrm{E}-02$ & $5.38 \mathrm{E}-10$ & $2.29 \mathrm{E}-01$ & --- & --- & --- & --- \\
\hline UE5n & CAMBRIC & 09-Sep-99 & $1.3 \mathrm{E}+05$ & $8.9 \mathrm{E}+05$ & $1.88 \mathrm{E}+01$ & $4.14 \mathrm{E}-02$ & $6.01 \mathrm{E}-10$ & $2.38 \mathrm{E}-01$ & $<40$ & $2.3 \mathrm{E}-03$ & $3.51 \mathrm{E}-09$ & --- \\
\hline RNM-1 & CAMBRIC & 03-Jun-04 & $3.4 \mathrm{E}+02$ & $3.1 \mathrm{E}+03$ & $1.22 \mathrm{E}+03$ & $2.44 \mathrm{E}+00$ & $1.38 \mathrm{E}-12$ & 4.42E-04 & --- & $<0.0034$ & 2.06E-07 & $5.96 \mathrm{E}-04$ \\
\hline RNM-1 & CAMBRIC & 28-Jun-00 & $2.8 \mathrm{E}+04$ & $2.1 \mathrm{E}+05$ & --- & --- & $1.06 \mathrm{E}-12$ & 4.30E-04 & --- & --- & --- & -- \\
\hline RNM-2S & CAMBRIC & 10-Jul-03 & $1.3 \mathrm{E}+05$ & $1.1 \mathrm{E}+06$ & $3.64 \mathrm{E}+02$ & 7.35E-01 & 2.30E-10 & $1.03 \mathrm{E}-01$ & --- & $1.5 \mathrm{E}-03$ & $6.16 \mathrm{E}-07$ & $1.35 \mathrm{E}-03$ \\
\hline RNM-2S & CAMBRIC & 09-May-03 & $1.5 \mathrm{E}+05$ & $1.3 \mathrm{E}+06$ & $3.87 \mathrm{E}+02$ & 7.07E-01 & $2.55 \mathrm{E}-10$ & $1.15 \mathrm{E}-01$ & --- & --- & 4.82E-07 & $9.07 \mathrm{E}-04$ \\
\hline RNM-2S & CAMBRIC & 14-Jun-00 & $1.9 \mathrm{E}+05$ & $1.4 \mathrm{E}+06$ & --- & -- & $1.62 \mathrm{E}-10$ & 7.92E-02 & --- & --- & -- & -- \\
\hline RNM-2S & CAMBRIC & 11-Oct-99 & $2.3 \mathrm{E}+05$ & $1.6 \mathrm{E}+06$ & $4.13 \mathrm{E}+02$ & $8.34 \mathrm{E}-01$ & 1.64E-10 & $7.40 \mathrm{E}-02$ & $<40$ & $1.3 \mathrm{E}-03$ & 2.12E-07 & $4.18 \mathrm{E}-04$ \\
\hline \multicolumn{13}{|c|}{ Hot Wells - Yucca Flat } \\
\hline U4u PS2a & DALHART & 9-Oct-03 & $2.7 \mathrm{E}+07$ & $6.2 \mathrm{E}+07$ & $1.56 \mathrm{E}+05$ & $3.26 \mathrm{E}+02$ & 1.74E-07 & $2.93 E+01$ & --- & $3.51 \mathrm{E}+01$ & 1.04E-04 & $1.32 \mathrm{E}-01$ \\
\hline U4u PS2a & DALHART & 16-Aug-99 & $1.6 \mathrm{E}+07$ & $3.1 \mathrm{E}+07$ & $1.19 \mathrm{E}+05$ & $2.29 \mathrm{E}+02$ & 4.45E-08 & $8.52 \mathrm{E}+00$ & --- & $1.31 \mathrm{E}+01$ & $5.32 \mathrm{E}-05$ & $2.85 \mathrm{E}-02$ \\
\hline UE-7ns & BOURBON & 21-Aug-01 & $4.6 \mathrm{E}+03$ & $3.2 \mathrm{E}+04$ & $6.97 \mathrm{E}+01$ & 1.39E-01 & $1.85 \mathrm{E}-12$ & $1.40 \mathrm{E}-03$ & --- & --- & 1.75E-07 & $6.05 \mathrm{E}-04$ \\
\hline UE-2ce & NASH & 12-Jul-05 & $9.3 \mathrm{E}+04$ & $8.3 E+05$ & $2.01 E+02$ & 8.15E-01 & $9.54 \mathrm{E}-10$ & 4.47E-01 & --- & $<2.4 \mathrm{e}-3$ & 5.02E-06 & 0.024 \\
\hline UE-2ce & NASH & 22-Aug-01 & $1.4 \mathrm{E}+05$ & $9.9 \mathrm{E}+05$ & $2.17 \mathrm{E}+02$ & 8.01E-01 & 1.62E-09 & $8.27 \mathrm{E}-01$ & --- & --- & 2.40E-05 & 0.032 \\
\hline U-3cn PS\#2 & BILBY & 09-Dec-04 & $7.9 \mathrm{E}+06$ & $8.0 \mathrm{E}+07$ & $1.15 \mathrm{E}+05$ & $3.72 \mathrm{E}+02$ & 4.46E-08 & $2.55 E+01$ & --- & $6.26 \mathrm{E}+01$ & 1.48E-04 & $2.52 \mathrm{E}-01$ \\
\hline U-3cn PS\#2 & BILBY & 18-Dec-01 & $9.9 \mathrm{E}+06$ & $8.6 \mathrm{E}+07$ & $8.78 \mathrm{E}+04$ & $3.03 E+02$ & $1.52 \mathrm{E}-07$ & $4.32 \mathrm{E}+01$ & --- & $8.26 \mathrm{E}+01$ & 7.38E-04 & $1.58 \mathrm{E}-01$ \\
\hline \multicolumn{13}{|c|}{ Hot Wells - Pahute Mesa } \\
\hline U20n PS1 DDh & CHESHIRE & 09-Jul-03 & $4.4 \mathrm{E}+07$ & $2.1 \mathrm{E}+08$ & $1.69 \mathrm{E}+05$ & $1.83 \mathrm{E}+02$ & 2.22E-09 & 7.99E-01 & --- & $1.23 \mathrm{E}+01$ & 2.74E-04 & $1.47 \mathrm{E}-01$ \\
\hline U20n PS1 DDh & CHESHIRE & 12-Oct-99 & $5.1 \mathrm{E}+07$ & $1.9 \mathrm{E}+08$ & $1.54 \mathrm{E}+05$ & $2.00 \mathrm{E}+02$ & 1.15E-09 & $4.20 \mathrm{E}-01$ & $2.77 \mathrm{E}+04$ & $2.23 \mathrm{E}+01$ & 4.91E-05 & 1.14E-01 \\
\hline U19ad PS1A & CHANCELLOR & 27-Sep-04 & $2.2 \mathrm{E}+07$ & $5.3 \mathrm{E}+07$ & $3.06 \mathrm{E}+05$ & $4.04 \mathrm{E}+02$ & $6.39 \mathrm{E}-09$ & $9.18 \mathrm{E}+00$ & --- & $4.60 \mathrm{E}+01$ & $5.59 \mathrm{E}-04$ & $1.94 \mathrm{E}+00$ \\
\hline U19q PS1d & CAMEMBERT & 16-Jul-03 & $1.1 \mathrm{E}+07$ & $5.4 \mathrm{E}+07$ & $4.92 \mathrm{E}+04$ & $2.93 \mathrm{E}+02$ & $7.73 \mathrm{E}-11$ & $1.84 \mathrm{E}-02$ & --- & --- & $2.85 \mathrm{E}-06$ & $1.98 \mathrm{E}-03$ \\
\hline U19q PS1d & CAMEMBERT & 21-Oct-98 & $2.1 \mathrm{E}+07$ & $7.8 \mathrm{E}+07$ & $1.07 \mathrm{E}+05$ & $1.31 \mathrm{E}+03$ & $5.28 \mathrm{E}-11$ & $1.81 \mathrm{E}-02$ & $1.10 \mathrm{E}+05$ & $8.4 \mathrm{E}-02$ & 2.27E-06 & 4.07E-03 \\
\hline U19v PS1ds & ALMENDRO & 23-Jul-03 & $1.4 \mathrm{E}+08$ & $7.7 \mathrm{E}+08$ & $2.99 \mathrm{E}+04$ & $7.44 \mathrm{E}+01$ & 3.20E-09 & $5.62 \mathrm{E}+00$ & --- & --- & $1.89 \mathrm{E}-03$ & $2.54 \mathrm{E}+00$ \\
\hline U19v PS1ds & ALMENDRO & 31-May-01 & $1.8 \mathrm{E}+08$ & $9.0 \mathrm{E}+08$ & $3.11 \mathrm{E}+04$ & $9.33 \mathrm{E}+01$ & 1.63E-09 & $3.58 \mathrm{E}+00$ & --- & --- & $1.54 \mathrm{E}-03$ & $2.33 \mathrm{E}+00$ \\
\hline U19v PS1ds & ALMENDRO & $26-S e p-00$ & $1.5 \mathrm{E}+08$ & $7.1 \mathrm{E}+08$ & $3.19 \mathrm{E}+04$ & $1.01 \mathrm{E}+02$ & $2.30 \mathrm{E}-09$ & $3.66 \mathrm{E}+00$ & --- & --- & 2.09E-03 & $2.76 \mathrm{E}+00$ \\
\hline U19v PS1ds & ALMENDRO & 18-Aug-99 & $1.6 \mathrm{E}+08$ & $6.8 \mathrm{E}+08$ & $2.47 \mathrm{E}+04$ & $3.56 \mathrm{E}+01$ & 1.60E-09 & $2.14 \mathrm{E}+00$ & --- & --- & 7.81E-03 & $1.39 \mathrm{E}+00$ \\
\hline ER-20-5 \#1 & TYBO/BENHAM $†$ & 30-Nov-04 & $3.8 \mathrm{E}+07$ & $2.0 \mathrm{E}+08$ & $9.63 \mathrm{E}+04$ & $2.24 \mathrm{E}+02$ & 4.39E-09 & $3.57 \mathrm{E}+00$ & --- & $3.5 \mathrm{E}-01$ & $1.99 \mathrm{E}-04$ & $1.92 \mathrm{E}-01$ \\
\hline ER-20-5 \#1 & TYBO/BENHAM $\dagger$ & 09-Jul-98 & $6.2 \mathrm{E}+07$ & $2.3 \mathrm{E}+08$ & $8.17 \mathrm{E}+04$ & $1.79 \mathrm{E}+02$ & 4.11E-09 & $3.32 \mathrm{E}+00$ & 502.00 & 2.7E-01 & 2.14E-04 & $2.68 \mathrm{E}-01$ \\
\hline ER-20-5 \#3 & TYBO/BENHAM $\dagger$ & 29-Nov-04 & $1.1 \mathrm{E}+05$ & $6.0 \mathrm{E}+05$ & $1.68 \mathrm{E}+03$ & $2.73 \mathrm{E}+00$ & 2.27E-11 & $1.31 \mathrm{E}-02$ & --- & 1.7E-02 & 1.66E-06 & $1.40 \mathrm{E}-03$ \\
\hline ER-20-5 \#3 & TYBO/BENHAM $\dagger$ & 15-Nov-01 & $1.4 \mathrm{E}+05$ & $6.3 \mathrm{E}+05$ & $1.57 \mathrm{E}+03$ & $2.08 \mathrm{E}+00$ & 3.49E-11 & $2.18 \mathrm{E}-02$ & --- & --- & 1.34E-05 & $1.20 \mathrm{E}-03$ \\
\hline ER-20-5 \#3 & TYBO/BENHAM † & 30-Apr-98 & $1.6 \mathrm{E}+05$ & $5.7 \mathrm{E}+05$ & $1.35 \mathrm{E}+03$ & $1.73 \mathrm{E}+00$ & $1.93 \mathrm{E}-11$ & 1.10E-02 & $<15$ & --- & --- & --. \\
\hline
\end{tabular}

† Both the TYBO and BENHAM tests are listed since the ER-20-5 well cluster was drilled in the near-field ( $300 \mathrm{~m}$ from the surface ground zero) environment of the TYBO test.

However, Pu isotopic signatures indicate the radionuclides in water samples from ER-20-5 wells are derived from the BENHAM test detonated $\sim 1300 \mathrm{~m}$ up-gradient (Kersting et al., 1999). 
Table 1.7 Radiochemical data

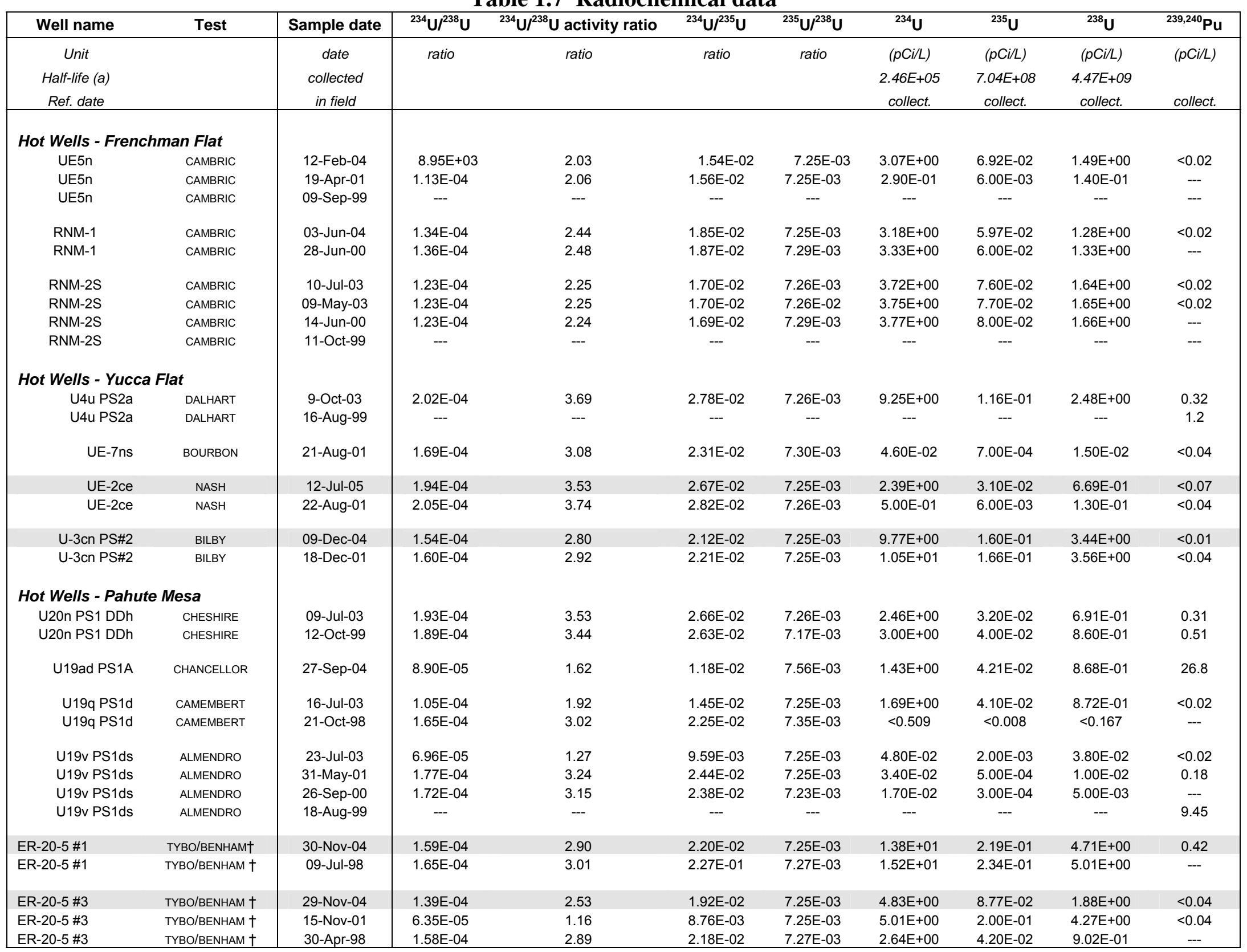

† Both the TYBO and BENHAM tests are listed since the ER-20-5 well cluster was drilled in the near-field ( $300 \mathrm{~m}$ from the surface ground zero) environment of the TYBO test.

However, Pu isotopic signatures indicate the radionuclides in water samples from ER-20-5 wells are derived from the BENHAM test detonated $\sim 1300 \mathrm{~m}$ up-gradient (Kersting et al., 1999). 


\title{
Chapter 2
}

\section{FY 2005 Environmental Well Data}

\author{
G.F. Eaton, V.Genetti, Q. (Max) Hu, G.B. Hudson, R.E. Lindvall, J. E. Moran, \\ E.C. Ramon, T.P. Rose, R.W. Williams, M. Zavarin, and P. Zhao \\ Chemical Biology and Nuclear Science Division \\ Lawrence Livermore National Laboratory
}

\subsection{Introduction}

This chapter summarizes the results of chemical and isotopic analyses of groundwater sampled collected from UGTA project environmental monitoring wells during FY 2005. Geochemical data gathered through this effort provide an independent means of evaluating groundwater flow model predictions for the NTS. The sampling program is a coordinated effort between the various UGTA contractors including Lawrence Livermore National Laboratory (LLNL), Los Alamos National Laboratory (LANL), the Desert Research Institute (DRI), the U.S. Geological Survey (USGS), Stoller-Navarro Joint Venture (SNJV), and Bechtel Nevada (BN). During FY 2005, groundwater characterization samples were collected from three environmental wells on Rainier Mesa: ER-12-1, ER-12-3 and ER-12-4 (Figure 2.1). The analytical results from these sampling events are compiled in Tables 2.1-2.7, and data interpretations are discussed below. Laboratory analytical protocols are fully described in the LLNL Standard Operating Procedures written in support of the UGTA Project (LLNL, 2004). 


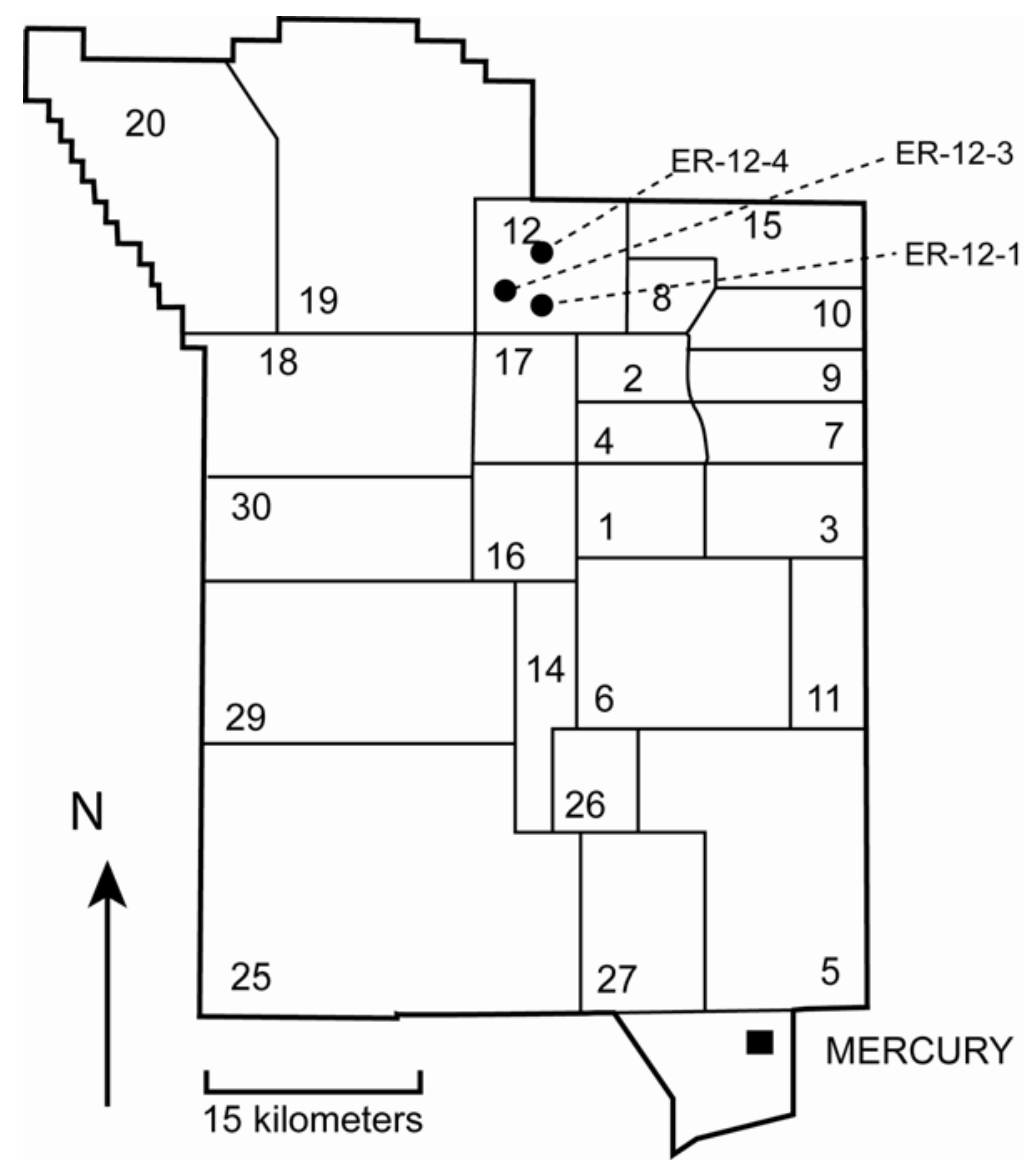

Figure 2.1 Map of the NTS showing environmental well sampling locations for FY2005.

\section{$2.2 \quad$ ER-12-1}

ER-12-1 is located on the eastern flank of Rainier Mesa along the U-12e tunnel access road, in Area 12 of the NTS. The well was drilled to a total depth of 1093.6 meters below ground surface in 1992, and was completed with five slotted intervals in Paleozoic carbonates and mudstones. Most the water is produced from the uppermost slotted interval (516 - 555 meters below ground surface) completed in the Sevy Dolomite (DOE/NV, 1996; Raytheon Services Nevada, 1992).

Approximately 19,600 gallons of water were purged from ER-12-1 prior to sampling on December 8, 2004, at an average pump rate of $\sim 29$ gallons per minute. LLNL participated in one previous groundwater sampling operation at ER-12-1 in January 1993 (LLNL, 1993).

ER-12-1 produces $\mathrm{Ca}-\mathrm{Mg}-\mathrm{HCO}_{3}$ groundwater with a near-neutral $\mathrm{pH}$ that is typical of the carbonate aquifer waters at the NTS, but with unusually high levels of sulfate (355 $\mathrm{mg} / \mathrm{L})$. The presence of large amounts of iron in the water $(5 \mathrm{mg} / \mathrm{L})$ may indicate pyrite oxidation, which would account for the high sulfate concentration. The stable isotope composition of the water $\left(\delta \mathrm{D}=-94.5 \% ; \delta^{18} \mathrm{O}=-12.6 \% \mathrm{o}\right)$ is very similar to reported 
values for perched water in the Rainier Mesa tunnels (Russell et al., 1987), and is substantially enriched in heavy isotopes compared to carbonate aquifer groundwaters found throughout most of Yucca Flat. These data suggest that ER-12-1 groundwater is derived from local recharge on Rainier Mesa.

The tritium activity in ER-12-1 groundwater $(3.2 \mathrm{pCi} / \mathrm{L})$ is more than a factor of 100 times lower than the measured activity in 1993 (361 pCi/L; LLNL, 1993). Since the halflife of ${ }^{3} \mathrm{H}$ is 12.3 years, we can assume the tritium that was observed in 1993 has either migrated away from the well bore or was removed by pumping. The original source of the tritium was speculated to be from the U12e tunnel effluent ponds, 500 meters to the west of the well site (DOE/NV, 1996). The ${ }^{3} \mathrm{He} /{ }^{4} \mathrm{He}$ ratio in the water $\left(6.20 \times 10^{-7}\right)$ is less than the ratio in ambient air $\left(1.38 \times 10^{-6}\right)$, indicating that the dissolved helium is dominated by a terrigenic ${ }^{4} \mathrm{He}$ source from the decay of uranium and thorium in surrounding host rocks. Assuming a radiogenic ${ }^{4} \mathrm{He}$ accumulation rate of $5 \times 10^{-11} \mathrm{~cm}^{3} / \mathrm{g}$ $\mathrm{H}_{2} \mathrm{O}$ per year, the apparent ${ }^{4} \mathrm{He}$ accumulation age is 3,000 years. A mean groundwater recharge temperature of $6.4^{\circ} \mathrm{C}$ was calculated from the dissolved $\mathrm{Ne}, \mathrm{Ar}, \mathrm{Kr}$, and $\mathrm{Xe}$ concentration data based on an assumed recharge altitude of $2,000 \mathrm{~m}$.

Dissolved inorganic carbon in ER-12-1 groundwater has a ${ }^{14} \mathrm{C}$ value of 11.0 percent modern carbon, yielding an uncorrected ${ }^{14} \mathrm{C}$ age of 18,200 years. The $\delta^{13} \mathrm{C}$ value of the water is unusually depleted in ${ }^{13} \mathrm{C}(-10.7 \%)$ implying very little equilibration with carbonate minerals in the aquifer. While this may indicate that the water had a long residence time in a different lithologic unit (other than carbonate rock), the high $\mathrm{Ca}-\mathrm{Mg}-\mathrm{HCO}_{3}$ concentrations in the water clearly indicate chemical equilibration with the carbonate host rock. The apparent lack of isotopic equilibration with the carbonate rock suggests that the uncorrected radiocarbon age may be close to the actual mean residence time of the groundwater.

ER-12-1 groundwater contains $17.7 \mathrm{mg} / \mathrm{L}$ chloride and has a high ${ }^{36} \mathrm{Cl} / \mathrm{Cl}$ ratio $\left(7.80 \times 10^{-13}\right)$ compared to the modern atmospheric ratio for southern Nevada $\left(\sim 5 \times 10^{-13}\right.$, Fabryka-Martin et al., 1993). The ${ }^{36} \mathrm{Cl}$ enrichment may reflect groundwater recharge during the last pluvial period (late Pleistocene), during which time the atmospheric production of ${ }^{36} \mathrm{Cl}$ was greater due to the reduced strength in the earth's magnetic field (Mazaud et al., 1991). This would imply a groundwater residence time in excess of 10,000 years, in accordance with the radiocarbon data. It should be noted, however, that the samples collected at ER-12-1 in 1993 contained much higher chloride (42 mg/L) and had a higher ${ }^{36} \mathrm{Cl} / \mathrm{Cl}$ ratio $\left(5.06 \times 10^{-12}\right)$, implying an anthropogenic source of chloride was present in the water (possibly from drilling fluid or $\mathrm{LiBr}$ tracers). If some residual amount of this material is still present in the surrounding aquifer matrix, it may account for the elevated ${ }^{36} \mathrm{Cl} / \mathrm{Cl}$ ratio in the 2004 sample.

The strontium concentration $(0.21 \mathrm{mg} / \mathrm{L})$ and ${ }^{87} \mathrm{Sr} /{ }^{86} \mathrm{Sr}$ ratio $(0.71230)$ in ER-12-1 groundwater is consistent with water-rock reactions with the carbonate host rock. Although most marine carbonate rocks have unaltered ${ }^{87} \mathrm{Sr} /{ }^{86} \mathrm{Sr}$ ratios in the range of 0.7078 to 0.7093 (Faure, 1986), Peterman et al. (1992) showed that high groundwater ${ }^{87} \mathrm{Sr} /{ }^{86} \mathrm{Sr}$ ratios are common in the carbonate aquifers of the Ash Meadows flow system. 
The elevated ${ }^{87} \mathrm{Sr} /{ }^{86} \mathrm{Sr}$ ratios in the carbonate rock may be related to an earlier episode of radiogenic ${ }^{87} \mathrm{Sr}$ enrichment from the underlying Precambrian clastic rocks.

The uranium concentration in ER-12-1 $(1.63 \mu \mathrm{g} / \mathrm{L})$ is within the range of expected values for LCA groundwater in southern Nevada (Paces et al., 2002), and the ${ }^{235} \mathrm{U} /{ }^{238} \mathrm{U}$ ratio indicates the uranium is natural in origin. The ${ }^{234} \mathrm{U} /{ }^{238} \mathrm{U}$-activity ratio (1.62) indicates a slight enrichment in ${ }^{234} \mathrm{U}$, suggesting some natural disequilibria between the solubility of ${ }^{234} \mathrm{U}$ and ${ }^{238} \mathrm{U}$ in the crystal lattice structure of uranium-bearing minerals in the aquifer host rock.

\subsection{ER-12-3}

Well ER-12-3 was constructed on Rainier Mesa and drilled into the carbonate aquifer with the objective of acquiring data on the radiological and hydrogeologic environment beneath Rainier Mesa. Well completion, development, lithology and hydraulic testing data are summarized in BN (2006a) and SNJV (2006a). ER-12-3 was drilled to a total depth of 1,496 meters on April 28, 2005 with two intervals of slotted casing in the Lower Carbonate Aquifer (LCA). The upper interval is between 1,095 and 1,160 meters and the lower interval is between 1,278 and 1,474 meters. Saturated rocks consist of Paleozoic dolomite in the upper slotted interval and Paleozoic limestone in the lower slotted interval. Both intervals have little apparent porosity but are populated with numerous hairline fractures mostly cemented by later stage calcite deposits (BN, 2006a; SNJV, 2006a). Importantly, annular space surrounding the slotted casing was not filled with sand or gravel. Furthermore, the slotted zones were not isolated from the overlying formation water. However, they were isolated from a perched water zone that was encountered between 420 and 808 meters. Water production rates from the dolomite and limestone sections are similar.

Groundwater characterization samples were collected from well ER-12-3 on July 6, 2005. At the time of sampling, the depth to water was measured at 950 meters, the pump rate was $29.6 \mathrm{gpm}$, and the cumulative purge volume was $\sim 6.2 \times 10^{5}$ gallons of water. Measured $\mathrm{Br}^{-}(<0.02 \mathrm{mg} / \mathrm{L})$ indicates that purge water effectively removed drilling fluids (doped with LiBr tracer) from the well.

Well ER-12-3 produces dilute mixed type $\mathrm{Ca}-\mathrm{Mg}-\mathrm{Na}-\mathrm{HCO}_{3}$ groundwater with a conductivity value of $306 \mu \mathrm{S} / \mathrm{cm}$ and a slightly alkaline $\mathrm{pH}$ value (8.2). Although the water chemistry is typical of the carbonate aquifer, the concentrations of dissolved constituents are more dilute than normally observed in LCA wells. The stable isotope composition of the water is $-106 \%$ for $\delta \mathrm{D}$ and $-14.5 \%$ for $\delta^{18} \mathrm{O}$. The stable isotope composition is unlike that of other northern Yucca Flat wells completed in the carbonate aquifer. Instead, it is isotopically lighter (i.e., more negative $\delta$-values), which may result from mixing with a combination of local volcanic aquifer paleo-waters (e.g. HTH \#1 type water) and Rainier Mesa tunnel waters. Interestingly, these results suggest that the groundwater is in poor communication with the northern Yucca Flat carbonate aquifer waters. 
The tritium activity of groundwater from well ER-12-3 was determined by the helium accumulation method (Surano et al., 1992). The tritium activity is only $0.5 \mathrm{pCi} / \mathrm{L}$ and is significantly less than the activity in other nearby wells on and near Rainier Mesa. The ${ }^{3} \mathrm{He} /{ }^{4} \mathrm{He}$ ratio in ER-12-3 groundwater $\left(\mathrm{R}=1.8 \times 10^{-7}\right)$ is much lower than the atmospheric helium isotope ratio $\left(R_{a}=1.38 \times 10^{-6}\right)$, giving an $R / R_{a}$ value of 0.13 . The low abundance of ${ }^{3} \mathrm{He}$ indicates the water never contained a large amount of tritium. Conversely, ${ }^{4} \mathrm{He}$ is moderately enriched relative to the equilibrium atmospheric helium solubility for this location, indicating the presence of excess helium from the $\alpha$-decay of $U$ and $T h$ in the host rock. Assuming a ${ }^{4} \mathrm{He}$ in-growth rate of $1.35 \times 10^{9}$ atoms/year, and correcting for the presence of non-equilibrium 'excess air' in the sample, the ${ }^{4} \mathrm{He}$ data imply a mean aquifer residence time on the order of 13,000 years.

Dissolved inorganic carbon (DIC) in ER-12-3 groundwater has a $\delta^{13} \mathrm{C}$ value is $-5.4 \%$ and a ${ }^{14} \mathrm{C}$ value of $2.9 \mathrm{pmc}$, yielding an uncorrected ${ }^{14} \mathrm{C}$ age of $\sim 28,300$ years. The low ${ }^{14} \mathrm{C}$ and moderately heavy $\delta^{13} \mathrm{C}$ are indicative of groundwater interaction with the carbonate host rock in which the well was completed. These water-rock reactions cause the measured ${ }^{14} \mathrm{C}$ age to appear older than the actual age of the water. Corrected radiocarbon ages can be calculated using chemical modeling programs (NETPATH or PHREEQC) to account for these reactions. It should be noted that the total dissolved inorganic carbon (TDIC) in ER-12-3 groundwater is only $125 \mathrm{mg} / \mathrm{L}$, which is at the lower end of measured carbonate aquifer TDIC values. This may reflect dilution during mixing with low-TDIC groundwater from the overlying volcanic units.

ER-12-3 groundwater has a dilute chloride concentration $(5.7 \mathrm{mg} / \mathrm{L})$ and $\mathrm{a}^{36} \mathrm{Cl} / \mathrm{Cl}$ ratio $\left(5.39 \times 10^{-13}\right)$ that is in the range for the modern atmospheric ratio for southern Nevada $\left(\sim 5 \times 10^{-13}\right.$, Fabryka-Martin et al., 1993). It is significantly higher than most LCA ${ }^{36} \mathrm{Cl} / \mathrm{Cl}$ ratios and may again be an indication of mixing with overlying perched volcanic aquifer waters.

The groundwater from ER-12-3 has a ${ }^{87} \mathrm{Sr}{ }^{86} \mathrm{Sr}$ ratio of 0.71055 and a $\delta^{87} \mathrm{Sr}$ value of +1.9 $\%$. The relatively low $\mathrm{Sr}$ concentration $(98 \mu \mathrm{g} / \mathrm{L})$ and isotope ratios are also suggestive of significant mixing between LCA and overlying perched volcanic aquifers. ${ }^{2}$ The concentration of dissolved uranium is $1.8 \mu \mathrm{g} / \mathrm{L}$. This is in the normal range of values for groundwater from the NTS, and the ${ }^{235} \mathrm{U} /{ }^{238} \mathrm{U}$ ratio shows the uranium is natural in origin. The ${ }^{234} \mathrm{U} /{ }^{238} \mathrm{U}$-activity ratio is 2.09 .

\section{$2.4 \quad$ ER-12-4}

Well ER-12-4 was constructed in Area 12 on Rainier Mesa and drilled into the carbonate aquifer with the objective of acquiring data on the radiological and hydrogeologic environment beneath Rainier Mesa. Well completion, development, lithology and hydraulic testing data are summarized in BN (2006b) and SNJV (2006b). ER-12-4 was completed to a total depth of 1,132 meters on June 1, 2005 with 7 intervals of slotted casing, each about 13 meters in length. The top of the uppermost slotted casing is at 948 meters and the bottom of the lowermost slotted casing is at 1,118 meters. Saturated rocks

\footnotetext{
${ }^{2}$ The value has been corrected since it was first reported in the ER-12-3 well report dated March 3, 2006.
} 
consist of some Paleozoic dolomite but primarily Paleozoic limestone with little apparent porosity but populated with numerous hairline fractures mostly cemented by later stage calcite deposits (BN, 2006b; SNJV, 2006b). Interestingly, significant water production was only observed in the limestone. Also, production rates were significantly lower than those observed in ER-12-3.

Groundwater characterization samples were collected from well ER-12-4 on August 16, 2005. At the time of sampling, the depth to water was measured at 889 meters, the pump rate was $10 \mathrm{gpm}$, and the cumulative purge volume was $\sim 4.9 \times 10^{4}$ gallons. Bromide tracer concentrations $(<0.02 \mathrm{mg} / \mathrm{L})$ suggest that the well was sufficiently purged prior to sampling. However, the total purge volume was ten times less than at ER-12-3.

Well ER-12-4 produces a dilute mixed type $\mathrm{Ca}-\mathrm{Mg}-\mathrm{Na}-\mathrm{HCO}_{3}$ groundwater with a low conductivity value $(196 \mu \mathrm{S} / \mathrm{cm})$ and a $\mathrm{pH}$ of 8.8 . The stable isotope composition of the water is $-103 \%$ for $\delta \mathrm{D}$ and $-13.7 \%$ for $\delta^{18} \mathrm{O}$. The stable isotope composition is not unlike that of other northern Yucca Flat wells completed in the carbonate aquifer. However, it is also quite similar to that observed in T-tunnel waters in the past. The major element composition suggests that a significant proportion of the water is derived from volcanic aquifers. This suggests that the groundwater may be mixed with perched waters located in the overlying tuffs.

The tritium activity of groundwater from well ER-12-4 was determined by the helium accumulation method (Surano et al., 1992). The tritium activity is $89.7 \mathrm{pCi} / \mathrm{L}$, and higher than the tritium activity from the deeper ER-12-3 well. It suggests a possible mixing with waters from overlying tuffs in which underground nuclear tests were located. Significant tritium may, however, be an artifact of drilling and the small purge volume (compared to the ER-12-3 sampling event), as described below.

Dissolved inorganic carbon (DIC) in ER-12-4 groundwater has a $\delta^{13} \mathrm{C}$ value is $-7.6 \%$ and a ${ }^{14} \mathrm{C}$ value of $6.9 \mathrm{pmc}$, yielding an uncorrected ${ }^{14} \mathrm{C}$ age of 21,530 years. The low ${ }^{14} \mathrm{C}$ and relatively heavy $\delta^{13} \mathrm{C}$ are indicative of the interaction with LCA carbonate rock in which the well was completed. It should be noted that the total dissolved inorganic carbon (TDIC) was only $84 \mathrm{mg} / \mathrm{L}$, which is at the lower end of measured LCA TDIC.

The ${ }^{4} \mathrm{He}$ value is quite low $\left(1.1 \times 10^{12}\right.$ atoms $\left./ \mathrm{g}\right)$. ${ }^{4} \mathrm{He}$ is not appreciably enriched relative to the equilibrium atmospheric helium solubility for this location. The ${ }^{4} \mathrm{He}$ result suggests that either the water is very young or that the water sampled was not representative of ambient waters in ER-12-4. ${ }^{3} \mathrm{He}$ value is also very low and inconsistent with the expected ingrowth from measured tritium concentrations in this sample. The ${ }^{3} \mathrm{He}$ value suggests that noble gases were partially lost during sampling, possibly as a result of the low production rates in this well. 
ER-12-4 groundwater has a chloride concentration of $9.1 \mathrm{mg} / \mathrm{L}$ and a ${ }^{36} \mathrm{Cl} / \mathrm{Cl}$ ratio $\left(5.6 \times 10^{-13}\right)$ which is in the normal range for the modern atmospheric ratio for southern Nevada $\left(\sim 5 \times 10^{-13}\right.$, Fabryka-Martin et al., 1993). It is significantly higher than most LCA ${ }^{36} \mathrm{Cl} / \mathrm{Cl}$ ratios and may, again, be an indication of mixing with overlying perched volcanic aquifer waters.

The groundwater from ER-12-4 has a ${ }^{87} \mathrm{Sr} /{ }^{86} \mathrm{Sr}$ ratio of 0.71065 and a $\delta^{87} \mathrm{Sr}$ value of $+2.04 \%$. The relatively low $\mathrm{Sr}$ concentration $(58 \mu \mathrm{g} / \mathrm{L})$ and isotope ratios are also suggestive of significant mixing with overlying perched volcanic aquifers. The concentration of dissolved uranium $(0.3 \mu \mathrm{g} / \mathrm{L})$ is on the lower end of the normal range of values for groundwater from the NTS, and the ${ }^{235} \mathrm{U} /{ }^{238} \mathrm{U}$ ratio shows the uranium is natural in origin. The ${ }^{234} \mathrm{U} /{ }^{238} \mathrm{U}$-activity ratio is 1.93 .

\subsection{References}

BN (2006a) Completion Report for Well ER-12-3 Corrective Action Unit 99: Rainier Mesa - Shoshone Mountain, DOE/NV/11718--1182, Bechtel-Nevada, Las Vegas, NV, May 2006.

BN (2006b) Completion Report for Well ER-12-4 Corrective Action Unit 99: Rainier Mesa - Shoshone Mountain, DOE/NV--1208, Bechtel-Nevada, Las Vegas, NV, May 2006.

DOE/NV (1996) Detection of Tritium in Well ER-12-1. Memo prepared by D.W. Duncan, Hydrology Program Manager, Office of Environmental Management, Department of Energy, Nevada Operations Office, 23 January 1996, 2 p.

Fabryka-Martin, J., Wightman, S.J., Murphy, W.J., Wickham, M.P., Caffee, M.W., Nimz, G.J., Southon, J.R., and Sharma, P. (1993) Distribution of chlorine-36 in the unsaturated zone at Yucca Mt.: An indicator of fast transport paths. FOCUS '93: Site Characterization and Model Validation, Las Vegas, NV, 26-29 Sept 1993.

Faure, G. (1986) Principles of Isotope Geology, $2^{\text {nd }}$ Edition. John Wiley \& Sons, New York, $589 \mathrm{p}$.

Ingraham, N.L., Lyles, B.F., Jacobson, R.L., and Hess, J.W. (1991) Stable isotopic study of precipitation and spring discharge in southern Nevada. Journal of Hydrology, v. 125 , p. $243-258$.

LLNL (1993) Isotopic Analyses of Groundwater from Monitoring Well ER-12-1. Isotope Hydrology Team, Nuclear Chemistry Division, Lawrence Livermore National Laboratory, 15 December 1993, 5 p.

LLNL (2004) Analytical Measurements: Standard Operating Procedures, Underground Test Area Project. Chemical Biology and Nuclear Science Division, Lawrence Livermore National Laboratory, 30 June 2004.

Mazaud, A., Laj, C., Bard, E., Arnold, M., and Tric, E. (1991) Geomagnetic field control of ${ }^{14} \mathrm{C}$ production over the last $80 \mathrm{ky}$ : Implications for the radiocarbon time-scale. Geophys. Res. Lett., v. 18, p. 1885-1888. 
Moran, J.E., and Rose, T.P. (2003) A chlorine-36 study of regional groundwater flow and vertical transport in southern Nevada. Environmental Geology, v. 43, p. 592-605.

Paces, J.B., Ludwig, K.R., Peterman, Z.E., and Neymark, L.A. (2002) ${ }^{234} U /{ }^{238} U$ evidence for local recharge and patterns of groundwater flow in the vicinity of Yucca Mountain, Nevada, USA. Applied Geochemistry, v. 17, p. 751-779.

Peterman, Z.E., Stuckless, J.S., Mahan, S.A., Marshall, B.D., Gutentag, E.D., and Downey, J.S. (1992) Strontium isotope characterization of the Ash Meadows groundwater system, southern Nevada, USA In: Y.K. Kharaka and A.S. Maest, eds., Proceedings of the $7^{\text {th }}$ International symposium on Water-Rock Interaction, 10-18 July 1992, Park City, Utah. A.A. Balkema, Rotterdam, p. 825-829.

Raytheon Services Nevada (1992) Well Diagrams for GCP Well ER-12-1. Memo prepared by D.R. Schwichtenberg, Drilling \& GCP Technical Support, Raytheon Services Nevada, 24 June 1992, 3 p.

Russell, C.E., Hess, J.W., and Tyler, S.W. (1987) Hydrogeologic investigations of flow in fractured tuffs, Rainier Mesa, Nevada Test Site. In: D.D. Evans and T.J. Nicholson, eds., Flow and Transport Through Unsaturated Fractured Rock. American Geophysical Union, Geophysical Monograph 42, AGU, Washington D.C., pp. 43-50.

SNJV (2006a) Analysis of ER-12-3 FY 2005 Hydrologic Testing, Nevada Test Site, Nye County, Nevada, S-N/99205-080, Rev. 0, Stoller-Navarro Joint Venture, Las Vegas, NV, July 2006.

SNJV (2006b) Analysis of FY 2005/2006 Hydrologic Testing and Sampling Results for Well ER-12-4, Nevada Test Site, Nye County, Nevada, S-N/99205--083, Rev. 0, Stoller-Navarro Joint Venture, Las Vegas, NV, September 2006.

Surano, K.A., Hudson, G.B., Failor, R.A., Sims, J.M., Holland, R.C., MacLean, S.C., and Garrison, J.C. (1992) Helium-3 mass spectrometry for low-level tritium analysis of environmental samples. Jour. Radioanal. Nuclear Chem. Articles, v. 161, p. 443-453. 
Table 2.1 Environmental well site information

\begin{tabular}{|c|c|c|c|c|c|c|c|c|c|c|c|c|}
\hline Well name & $\begin{array}{c}\text { Test } \\
\text { Name }\end{array}$ & $\begin{array}{l}\text { Test } \\
\text { Date }\end{array}$ & Latitude & Longitude & $\begin{array}{c}\text { Surface } \\
\text { Elevation }\end{array}$ & $\begin{array}{c}\text { Well } \\
\text { Depth }\end{array}$ & Open Interval & $\begin{array}{l}\text { Water } \\
\text { Depth }\end{array}$ & $\begin{array}{l}\text { Sample } \\
\text { Method }\end{array}$ & $\begin{array}{c}\text { Volume } \\
\text { Pumped }\end{array}$ & $\begin{array}{c}\text { Sample } \\
\text { Depth }\end{array}$ & $\begin{array}{c}\text { Sample } \\
\text { date }\end{array}$ \\
\hline Units & & & $(d m s)$ & $(d m s)$ & $(f t)$ & (ft bgs) & (ft bgs) & (ft bgs) & & $(g a l)$ & (ft bgs) & \\
\hline \multicolumn{13}{|c|}{ Clean Wells - Frenchman Flat } \\
\hline Water Well 5a & --- & --- & 364635 & 1155729 & 3092 & 910 & $642-877$ & 695 & pump & --- & -- & 14-Aug-00 \\
\hline Water Well $5 c$ & --- & --- & 364708 & 1155744 & 3081 & 1200 & $887-1187$ & 689 & pump & -- & --- & 07-Aug-00 \\
\hline Water Well 5b & --- & --- & 364805 & 1155808 & 3093 & 900 & $700-900$ & 683 & pump & --- & --- & 07-Aug-00 \\
\hline ER-5-4 & --- & --- & 364928 & 1155748 & 3127 & 3732 & $1770-2113 ; 3136-3350$ & 726 & pump & $3.74 \mathrm{E}+06$ & --- & 05-Jul-01 \\
\hline ER-5-4 \#2 & -- & -- & 364927 & 1155748 & 3127 & 7000 & $6486-6658$ & 697 & pump & $4.00 \mathrm{E}+06$ & --- & 21-Nov-02 \\
\hline UE-5c WW & -- & -- & 365011 & 1155847 & 3216 & 2682 & $1100-2682$ & 806 & pump & -- & --- & 08-Aug-00 \\
\hline UE-5 PW-3 & --- & --- & 365201 & 1155816 & 3297 & 955 & 891-955 & 891 & pump & --- & --- & 09-Aug-00 \\
\hline ER-5-3 & -- & --- & 365223 & 1155617 & 3334 & 2606 & $1480-1737 ; 2420-2549$ & 927 & pump & $3.16 \mathrm{E}+06$ & --- & 28-Mar-01 \\
\hline ER-5-3 \#2 & --- & --- & 365223 & 1155618 & 3334 & 5683 & $4674-4868$ & 952 & pump & $3.46 \mathrm{E}+06$ & --- & 17-May-01 \\
\hline Water Well 4a & -- & -- & 365412 & 1160139 & 3604 & 1517 & $944-1502$ & 835 & pump & -- & --- & 08-Aug-00 \\
\hline \multicolumn{13}{|c|}{ Clean Wells - Yucca Flat } \\
\hline ER-2-1 & -- & -- & 370731 & 1160342 & 4222 & 2600 & $1642-2076$ & 1723.0 & pump & $2.15 \mathrm{E}+04$ & --- & 03-Sep-03 \\
\hline ER-6-1 \#2 & -- & --- & 365901 & 1155935 & 3934 & 3200 & $1775-3200$ & 1545 & pump & $3.80 \mathrm{E}+06$ & -- & 16-Jan-03 \\
\hline ER-6-2 & --- & -- & 365739 & 1160434 & 4231 & 3430 & $1746-3430$ & 1785 & pump & $3.37 \mathrm{E}+06$ & 1904 & 04-Aug-04 \\
\hline ER-7-1 & -- & --- & 370424 & 1155943 & 4247 & 2500 & $2182-2479$ & 1854.0 & pump & $3.54 \mathrm{E}+07$ & --- & 17-Jul-03 \\
\hline \multicolumn{13}{|c|}{ Clean Wells - Rainier Mesa } \\
\hline ER-12-1 & -- & --- & 371106 & 1161103 & 5817 & 3588 & $1641-1846$ & 1538 & pump & $1.96 \mathrm{E}+04$ & -- & 08-Dec-04 \\
\hline ER-12-2 & -- & -- & 371019 & 1160721 & 4705 & 6883 & $2958-6883$ & 191 & pump & $3.52 E+05$ & --- & 01-Apr-03 \\
\hline ER-12-3 & --- & -- & 371142 & 1161250 & 7385 & 4908 & $3591-3805 ; 4919-4834$ & 3100 & pump & $6.32 E+05$ & -- & 6-Jul-05 \\
\hline ER-12-4 & --- & --- & 371311 & 1161059 & 6883 & 3715 & $3111-3669$ & 2600 & pump & $4.91 \mathrm{E}+04$ & --- & 16-Aug-05 \\
\hline
\end{tabular}


Table 2.1 Environmental well site information (continued)

\begin{tabular}{|c|c|c|c|c|c|c|c|c|c|c|c|c|}
\hline Well name & $\begin{array}{c}\text { Test } \\
\text { Name } \\
\end{array}$ & $\begin{array}{l}\text { Test } \\
\text { Date } \\
\end{array}$ & Latitude & Longitude & $\begin{array}{c}\text { Surface } \\
\text { Elevation }\end{array}$ & $\begin{array}{c}\text { Well } \\
\text { Depth }\end{array}$ & Open Interval & $\begin{array}{l}\text { Water } \\
\text { Depth }\end{array}$ & $\begin{array}{l}\text { Sample } \\
\text { Method }\end{array}$ & $\begin{array}{l}\text { Volume } \\
\text { Pumped }\end{array}$ & $\begin{array}{c}\text { Sample } \\
\text { Depth }\end{array}$ & $\begin{array}{c}\text { Sample } \\
\text { date }\end{array}$ \\
\hline Units & & & $(d m s)$ & $(d m s)$ & (ft) & (ft bgs) & (ft bgs) & (ft bgs) & & (gal) & (ft bgs) & \\
\hline \multicolumn{13}{|c|}{ Clean Wells - Pahute Mesa-Oasis Valley } \\
\hline ER-EC-1 & --- & --- & 371223 & 1163144 & 6026 & 5000 & $2298-2821 ; 3348-3760 ; 4449-4750$ & 1859 & pump & $2.02 E+05$ & --- & 3-Jun-03 \\
\hline ER-EC-1 & --- & --- & 371223 & 1163144 & 6026 & 5000 & $2298-2821 ; 3348-3760 ; 4449-4750$ & 1859 & pump & $2.80 \mathrm{E}+06$ & --- & 1-Feb-00 \\
\hline ER-EC-2A & --- & --- & 370852 & 1163405 & 4904 & 4974 & $1707-2179 ; 3077-3549 ; 4487-4916$ & 748 & pump & $2.66 \mathrm{E}+04$ & --- & 8-Jul-03 \\
\hline ER-EC-2A & --- & --- & 370852 & 1163405 & 4904 & 4974 & $1707-2179 ; 3077-3549 ; 4487-4916$ & 748 & pump & $2.34 \mathrm{E}+06$ & --- & 27-Jul-00 \\
\hline ER-EC-4 & --- & --- & 370939 & 1163752 & 4760 & 3487 & $989-1224 ; 1910-2253 ; 3103-3404$ & 749 & pump & $1.23 \mathrm{E}+05$ & --- & 24-Jun-03 \\
\hline ER-EC-4 & --- & --- & 370939 & 1163752 & 4760 & 3487 & $989-1224 ; 1910-2253 ; 3103-3404$ & 749 & pump & $2.84 \mathrm{E}+06$ & --- & 17-Aug-00 \\
\hline ER-EC-5 & --- & --- & 370505 & 1163349 & 5077 & 2500 & 1197-1399; 1892-2094; 2246-2417 & 1017 & pump & $2.21 \mathrm{E}+05$ & --- & 15-Jul-03 \\
\hline ER-EC-5 & --- & --- & 370505 & 1163349 & 5077 & 2500 & 1197-1399; 1892-2094; 2246-2417 & 1017 & pump & $3.50 \mathrm{E}+06$ & --- & 25-May-00 \\
\hline ER-EC-6 & --- & --- & 371126 & 1162948 & 5605 & 5000 & $1628-1871 ; 2195-2507 ; 3438-3811 ; 4421-4904$ & 1426 & pump & $2.15 \mathrm{E}+05$ & --- & 10-Jun-03 \\
\hline ER-EC-6 & -- & --- & 371126 & 1162948 & 5605 & 5000 & $1628-1871 ; 2195-2507 ; 3438-3811 ; 4421-4904$ & 1426 & pump & $2.2 \mathrm{E}+05$ & -- & $10-F e b-00$ \\
\hline ER-EC-7 & --- & -- & 365910 & 1162841 & 4800 & 1386 & $920-979 ; 1215-1304$ & 719 & pump & $2.90 \mathrm{E}+05$ & --- & 21-Jul-03 \\
\hline ER-EC-7 & --- & --- & 365910 & 1162841 & 4800 & 1386 & $920-979 ; 1215-1304$ & 719 & pump & $3.60 \mathrm{E}+06$ & -- & 5-Jun-00 \\
\hline ER-EC-8 & --- & --- & 370617 & 1163753 & 4245 & 2000 & $683-984 ; 1447-1507 ; 1677-1908$ & 323 & pump & $2.34 \mathrm{E}+05$ & -- & 1-Jul-03 \\
\hline ER-EC-8 & --- & -- & 370617 & 1163753 & 4245 & 2000 & $683-984 ; 1447-1507 ; 1677-1908$ & 323 & pump & $3.82 \mathrm{E}+06$ & -- & 12-Jul-00 \\
\hline ER-18-2 & --- & --- & 370621 & 1162222 & 5436 & 2500 & 1930-1960; 2000-2030; 2071-2101 & 1213 & pump & $9.76 \mathrm{E}+04$ & --- & 17-Jun-03 \\
\hline ER-18-2 & --- & --- & 370621 & 1162222 & 5436 & 2500 & 1930-1960; 2000-2030; 2071-2101 & 1213 & pump & $2.22 \mathrm{E}+05$ & -- & 21-Mar-00 \\
\hline
\end{tabular}


Table 2.2 Environmental well field parameter and anion data

\begin{tabular}{|c|c|c|c|c|c|c|c|c|c|c|c|}
\hline Well name & Test & $\begin{array}{c}\text { Sample } \\
\text { date }\end{array}$ & $\mathrm{pH}$ & $T$ & Cond. & $\begin{array}{c}\text { TDIC as } \\
\mathrm{HCO}_{3}{ }^{-} \\
\end{array}$ & $\mathbf{F}$ & $\mathrm{Cl}$ & $\mathrm{Br}$ & $\mathrm{NO}_{3}$ & $\mathrm{SO}_{4}$ \\
\hline Units & & date & & $\left({ }^{\circ} \mathrm{C}\right)$ & $(\mu \mathrm{S} / \mathrm{cm})$ & $(\mathrm{mg} / \mathrm{L})$ & $(m g / L)$ & $(m g / L)$ & $(m g / L)$ & $(\mathrm{mg} / \mathrm{L})$ & $(\mathrm{mg} / \mathrm{L})$ \\
\hline \multicolumn{12}{|c|}{ Clean Wells - Frenchman Flat } \\
\hline Water Well 5a & --- & 14-Aug-00 & 8.9 & 22.3 & 600 & 313 & 1.1 & 11.1 & $<0.2$ & 3.7 & 28.5 \\
\hline Water Well 5c & --- & 07-Aug-00 & 8.7 & 28.2 & 601 & 306 & 1.0 & 11.3 & $<0.2$ & 7.4 & 29.3 \\
\hline Water Well 5b & --- & 07-Aug-00 & 8.5 & 24.6 & 512 & 171 & 0.7 & 23.4 & $<0.2$ & 12.8 & 55.0 \\
\hline ER-5-4 & --- & 05-Jul-01 & 8.7 & 30.2 & 885 & 313 & 6.2 & 26.8 & $<0.3$ & 4.0 & 120.0 \\
\hline ER-5-4 \#2 & --- & 21-Nov-02 & 8.7 & 38.1 & 1249 & 394 & 63.9 & 51.7 & $<0.5$ & $<0.1$ & 119.4 \\
\hline UE-5c WW & --- & 08-Aug-00 & 8.5 & 25.8 & 463 & 179 & 1.8 & 12.8 & $<0.2$ & 7.5 & 43.7 \\
\hline UE-5 PW-3 & --- & 09-Aug-00 & 8.3 & 21.5 & 371 & 153 & 0.9 & 9.4 & $<0.2$ & 14.6 & 31.8 \\
\hline ER-5-3 & --- & 28-Mar-01 & 8.3 & 30.0 & 445 & 169 & 2.5 & 15.5 & $<0.1$ & 7.0 & 40.0 \\
\hline ER-5-3 \#2 & --- & 17-May-01 & 6.7 & 33.8 & 1158 & 797 & 1.3 & 38.0 & $<0.1$ & $<0.09$ & 64.0 \\
\hline Water Well 4a & --- & 08-Aug-00 & 7.9 & 26.5 & 409 & 157 & 1.2 & 13.2 & $<0.2$ & 17.4 & 39.8 \\
\hline \multicolumn{12}{|c|}{ Clean Wells - Yucca Flat } \\
\hline ER-2-1 & --- & 03-Sep-03 & 9.3 & 21.3 & 368 & 189 & 1.8 & 4.4 & $<0.03$ & 2.4 & 15.9 \\
\hline ER-6-1 \#2 & --- & 16-Jan-03 & 7.6 & 39.9 & 408 & 256 & 0.8 & 10.0 & 0.8 & 1.1 & 34.0 \\
\hline ER-6-2 & -- & 04-Aug-04 & 7.5 & 34.9 & 617 & 433 & 1.4 & 23.9 & 0.1 & 1.4 & 52.4 \\
\hline ER-7-1 & --- & 17-Jul-03 & 7.6 & 49.4 & 488 & 243.5 & 0.8 & 9.5 & 0.1 & 0.1 & 34.4 \\
\hline \multicolumn{12}{|c|}{ Clean Wells - Rainier Mesa } \\
\hline ER-12-1 & --- & 08-Dec-04 & 7.5 & 25.0 & 984 & 257 & 0.3 & 17.7 & 0.4 & 0.1 & 355.4 \\
\hline ER-12-2 & --- & 01-Apr-03 & 8.1 & 35.2 & 528 & 303 & 2.2 & 7.0 & 0.7 & $<0.2$ & 27.4 \\
\hline ER-12-3 & --- & 06-Jul-05 & 8.2 & 30.6 & 306 & 125 & 1.5 & 5.7 & $<0.02$ & 0.8 & 24.6 \\
\hline ER-12-4 & --- & 16-Aug-05 & 8.8 & 23.9 & 196 & 84 & 0.3 & 9.1 & $<0.02$ & 8.5 & 11.4 \\
\hline \multicolumn{12}{|c|}{ Clean Wells - Pahute Mesa-Oasis Valley } \\
\hline ER-EC-1 & --- & 3-Jun-03 & 8.1 & 34.8 & 782 & 148.9 & 2.3 & 97.0 & 1.4 & 2.1 & 119.0 \\
\hline ER-EC-1 & --- & 1-Feb-00 & 7.9 & 37.0 & 818 & 148.5 & 2.4 & 97.0 & 1.1 & 2.5 & 145.0 \\
\hline ER-EC-2A & --- & 8-Jul-03 & 8.1 & 35.2 & 616 & 164.7 & 3.9 & 55.5 & 1.1 & 1.9 & 84.5 \\
\hline ER-EC-2A & -- & 27-Jul-00 & 7.8 & 40.4 & 706 & 170.5 & 5.9 & 63.0 & 0.6 & 1.2 & 99.0 \\
\hline ER-EC-4 & --- & 24-Jun-03 & 7.8 & 35.9 & 750 & 150.4 & 3.0 & 80.6 & 1.2 & 2.4 & 109.0 \\
\hline ER-EC-4 & --- & 17-Aug-00 & 7.9 & 38.5 & 793 & 158.1 & 3.6 & 95.7 & 1.3 & 3.2 & 130.0 \\
\hline ER-EC-5 & --- & 15-Jul-03 & 7.9 & 29.7 & 412 & 180.4 & 4.3 & 15.9 & 0.7 & 1.7 & 36.3 \\
\hline ER-EC-5 & --- & 25-May-00 & 7.9 & 29.9 & 424 & 175.7 & 4.6 & 16.1 & n.d. & 1.2 & 35.0 \\
\hline ER-EC-6 & --- & 10-Jun-03 & 8.1 & 33.9 & 516 & 147.0 & 2.7 & 51.7 & 0.9 & 2.0 & 75.4 \\
\hline ER-EC-6 & -- & $10-F e b-00$ & 8.1 & 37.9 & 613 & 153.2 & 3.1 & 44.0 & 0.8 & 2.0 & 56.0 \\
\hline ER-EC-7 & --- & 21-Jul-03 & 8.0 & 27.3 & 263 & 86.5 & 1.2 & 3.8 & $<0.03$ & 5.6 & 13.6 \\
\hline ER-EC-7 & --- & 5-Jun-00 & 7.9 & 30.0 & 315 & 118.8 & 1.3 & 5.2 & n.d. & 5.8 & 15.0 \\
\hline ER-EC-8 & --- & 1-Jul-03 & 8.1 & 36.7 & 642 & 173.8 & 5.2 & 47.3 & 1.0 & 1.4 & 76.1 \\
\hline ER-EC-8 & -- & 12-Jul-00 & 8.0 & 38.2 & 647 & 172.1 & 5.5 & 57.6 & 0.4 & 1.3 & 94.0 \\
\hline ER-18-2 & --- & 17-Jun-03 & 7.9 & 43.0 & 1277 & 881.0 & 12.5 & 12.3 & $<0.2$ & $<0.2$ & 52.9 \\
\hline ER-18-2 & --- & 21-Mar-00 & 7.6 & 55.2 & 1439 & 871.0 & 12.6 & 13.3 & n.d. & $<1.0$ & 53.0 \\
\hline
\end{tabular}


Table 2.3 Environmental well cations and metals data

\begin{tabular}{|c|c|c|c|c|c|c|c|c|c|c|c|c|c|c|c|c|c|c|c|c|c|}
\hline Well name & Test & Sample date & $\mathrm{Na}$ & K & $\mathrm{Ca}$ & $\mathrm{Mg}$ & Li & Al & Si & $\mathrm{Fe}$ & Mn & As & $\mathrm{Se}$ & $\mathrm{Sr}$ & Mo & $\mathrm{Sb}$ & I & $\mathrm{Ba}$ & $\mathrm{Pb}$ & $u$ & $\mathrm{Pu}$ \\
\hline Unit & & & $(\mathrm{mg} / \mathrm{L})$ & $(\mathrm{mg} / \mathrm{L})$ & $(\mathrm{mg} / \mathrm{L})$ & $(\mathrm{mg} / \mathrm{L})$ & $(\mathrm{mg} / \mathrm{L})$ & $(\mathrm{mg} / \mathrm{L})$ & $(\mathrm{mg} / \mathrm{L})$ & $(\mathrm{mg} / \mathrm{L})$ & $(\mu g / L)$ & $(\mu g / L)$ & $(\mu g / L)$ & $(\mu g / L)$ & $(\mu g / L)$ & $(\mu g / L)$ & $(\mu g / L)$ & $(\mu g / L)$ & $(\mu g / L)$ & $(\mu g / L)$ & $(\mathrm{pg} / \mathrm{L})$ \\
\hline \multicolumn{22}{|c|}{ Clean Wells - Frenchman Flat } \\
\hline Water Well 5a & --- & 14-Aug-00 & 147.0 & 5.5 & 1.7 & 0.8 & --- & $<0.05$ & 18.8 & $<0.04$ & $<6$ & 37 & $<24$ & $<13$ & 8.3 & --- & --- & 1 & $<14$ & --- & --- \\
\hline Water Well 5c & --- & 07-Aug-00 & 149.0 & 7.0 & 1.9 & 0.7 & --- & $<0.05$ & 25 & $<0.04$ & $<6$ & 32 & $<24$ & $<13$ & 7.3 & --- & --- & 1 & $<14$ & --- & --- \\
\hline Water Well 5b & --- & 07-Aug-00 & 101.0 & 12.6 & 7.3 & 2.5 & --- & $<0.05$ & 26.3 & $<0.04$ & $<6$ & $<20$ & $<24$ & $<13$ & 3.2 & --- & --- & 2 & $<14$ & --- & --- \\
\hline ER-5-4 & --- & 05-Jul-01 & 124.0 & 7.1 & 2.2 & 0.2 & --- & 0.2 & 24.5 & 0 & $<6$ & 47 & $<24$ & 35 & 63 & --- & --- & 8 & 21 & 1.60 & --- \\
\hline ER-5-4 \#2 & --- & 21-Nov-02 & 334.0 & 4.3 & 0.7 & 0.3 & 0.07 & 1.9 & 34.7 & 1 & 98 & $<20$ & $<24$ & 3.3 & 82 & --- & --- & 8 & $<14$ & 36.40 & --- \\
\hline UE-5c WW & --- & 08-Aug-00 & 100.0 & 6.7 & 6.9 & 1.8 & --- & 0.6 & 33.3 & 0 & $<6$ & $<20$ & $<24$ & 33 & 11 & --- & --- & 89 & $<14$ & --- & --- \\
\hline UE-5 PW-3 & --- & 09-Aug-00 & 61.8 & 5.0 & 16.4 & 6.7 & --- & $<0.05$ & 27.6 & $<0.04$ & $<6$ & $<20$ & $<24$ & 120 & 3.8 & --- & --- & 13 & $<14$ & --- & --- \\
\hline ER-5-3 & --- & 28-Mar-01 & 78.9 & 4.0 & 14.3 & 3.9 & --- & --- & --- & --- & $<6$ & 30 & $<24$ & 64 & 6.9 & --- & --- & 3 & $<14$ & 0.97 & --- \\
\hline ER-5-3 \#2 & --- & 17-May-01 & 145.0 & 15.8 & 86.1 & 32.6 & --- & --- & --- & --- & 92 & 35 & $<24$ & 1018 & 4.3 & --- & -- & 230 & $<14$ & 0.50 & --- \\
\hline Water Well 4a & --- & 08-Aug-00 & 57.3 & 6.2 & 23.1 & 8.3 & --- & $<0.05$ & 28.0 & $<0.04$ & $<6$ & $<20$ & $<24$ & 130 & 5.8 & --- & --- & 3 & $<14$ & --- & --- \\
\hline \multicolumn{22}{|c|}{ Clean Wells - Yucca Flat } \\
\hline ER-2-1 & --- & 03-Sep-03 & 73.0 & 3.8 & 3.1 & 0.3 & 0.02 & 0.1 & 55.8 & 2.20 & 110 & $<20$ & 40 & 6.7 & $<3$ & --- & 5.3 & $\begin{array}{c}9 \\
<1\end{array}$ & $<14$ & 3.40 & --- \\
\hline ER-6-1 \#2 & --- & 16-Jan-03 & 47.1 & 6.3 & 33.6 & 14.0 & 0.06 & $<0.05$ & 16.4 & $<0.04$ & 7 & $<20$ & $<24$ & 208 & $<3$ & --- & --- & 160 & $<14$ & 3.21 & --- \\
\hline ER-6-2 & --- & 04-Aug-04 & 72.1 & 10.2 & 67.4 & 22.3 & 0.18 & 0.01 & 40.1 & 0.03 & 38.3 & 38 & 0.905 & 231 & 5.4 & 3.15 & --- & 154 & 0.35 & 2.17 & --- \\
\hline ER-7-1 & --- & 17-Jul-03 & 41.6 & 5.6 & 27.1 & 13.3 & 0.04 & $<0.05$ & 18.3 & $<0.04$ & $<6$ & $<20$ & $<24$ & 156 & 10 & --- & 8.1 & 230 & $<14$ & 1.42 & --- \\
\hline \multicolumn{22}{|c|}{ Clean Wells - Rainier mesa } \\
\hline ER-12-1 & --- & 08-Dec-04 & 38.3 & 3.0 & 88.4 & 58.8 & 0.26 & $3 \mathrm{E}-04$ & 29.34 & 4.99 & 141 & 106 & 0.457 & 210 & 10.6 & 0.48 & -- & 35 & $\mathrm{BD}$ & 1.63 & --- \\
\hline ER-12-2 & --- & 01-Apr-03 & 117.2 & 2.1 & 6.5 & 2.1 & 0.19 & $<0.05$ & 6.9 & 0.26 & 22 & $<20$ & $<24$ & 317 & 4.7 & --- & --- & 210 & $<14$ & 0.02 & --- \\
\hline ER-12-3 & --- & 06-Jul-05 & 30.7 & 2.2 & 13.8 & 7.9 & $<0.03$ & 0.003 & 6.8 & 0.3 & 26.6 & 7.5 & $<1.5$ & 102 & 5.9 & 0.36 & 3.59 & 24.88 & 0.3 & 1.83 & --- \\
\hline ER-12-4 & --- & 16-Aug-05 & 28.3 & 3.1 & 8.4 & 3.5 & $<0.005$ & 0.02 & 3.53 & 1.0 & 45.3 & $<4.2$ & $<1.5$ & 63 & 2 & $<0.3$ & -- & 12.6 & 0.24 & 0.30 & --- \\
\hline \multicolumn{22}{|c|}{ Clean Wells - Pahute Mesa-Oasis Valley } \\
\hline ER-EC-1 & --- & 3-Jun-03 & 143.7 & 4.9 & 18.7 & 0.4 & 0.11 & 0.2 & 28.7 & $<0.04$ & $<6$ & $<20$ & $<24$ & 16 & $<3$ & --- & --- & & $<14$ & 9.51 & --- \\
\hline ER-EC-1 & --- & 1-Feb-00 & 154.0 & 6.0 & 19.0 & 0.4 & 0.15 & $<0.4$ & 23 & 0.64 & --- & --- & --- & 27 & --- & --- & -- & --- & --- & 8.12 & --- \\
\hline ER-EC-2A & --- & 8-Jul-03 & 127.8 & 2.2 & 8.7 & 0.2 & 0.15 & 0.1 & 26.5 & $<0.04$ & $<6$ & $<20$ & $<24$ & 28 & 6.8 & --- & --- & 15 & $<14$ & 7.05 & --- \\
\hline ER-EC-2A & --- & 27-Jul-00 & 123.0 & 2.5 & 13.1 & 2.5 & 0.14 & $<0.11$ & 15 & 0.25 & -- & --- & -- & 74 & --- & --- & --- & --- & -- & 9.20 & --- \\
\hline ER-EC-4 & --- & 24-Jun-03 & 118.7 & 8.1 & 26.1 & 5.0 & 0.09 & 0.2 & 40.3 & 0.05 & $<6$ & $<20$ & $<24$ & 136 & 4.7 & --- & --- & 2 & $<14$ & 4.08 & --- \\
\hline ER-EC-4 & --- & 17-Aug-00 & 116.0 & 8.7 & 25.9 & 3.8 & 0.09 & $<0.11$ & 21 & 0.18 & --- & --- & --- & 160 & --- & --- & -- & --- & --- & 4.10 & --- \\
\hline
\end{tabular}


Table 2.3 Environmental well cations and metals data (continued)

\begin{tabular}{|c|c|c|c|c|c|c|c|c|c|c|c|c|c|c|c|c|c|c|c|c|c|}
\hline Well name & Test & $\begin{array}{c}\text { Sample } \\
\text { date }\end{array}$ & $\mathrm{Na}$ & $\mathrm{K}$ & $\mathrm{Ca}$ & $\mathbf{M g}$ & $\mathrm{Li}$ & Al & $\mathrm{Si}$ & $\mathrm{Fe}$ & $\mathrm{Mn}$ & As & Se & $\mathrm{Sr}$ & Mo & Sb & I & $\mathrm{Ba}$ & $\mathrm{Pb}$ & u & $\mathrm{Pu}$ \\
\hline Unit & & & $(\mathrm{mg} / \mathrm{L})$ & $(m g / L)$ & $(\mathrm{mg} / \mathrm{L})$ & $(\mathrm{mg} / \mathrm{L})$ & $(m g / L)$ & $(\mathrm{mg} / \mathrm{L})$ & $(\mathrm{mg} / \mathrm{L})$ & $(\mathrm{mg} / \mathrm{L})$ & $(\mu g / L)$ & $(\mu g / L)$ & $(\mu g / L)$ & $(\mu g / L)$ & $(\mu g / L)$ & $(\mu g / L)$ & $(\mu g / L)$ & $(\mu g / L)$ & $(\mu g / L)$ & $(\mu g / L)$ & (pg/L) \\
\hline \multicolumn{22}{|c|}{ Clean Wells - Pahute Mesa-Oasis Valley } \\
\hline ER-EC-5 & --- & 15-Jul-03 & 70.8 & 1.1 & 19.8 & 0.8 & 0.09 & $<0.05$ & 24.5 & $<0.04$ & $<6$ & $<20$ & $<24$ & 128 & 6.2 & --- & --- & 3 & $<14$ & 3.34 & --- \\
\hline ER-EC-5 & --- & 25-May-00 & 75.0 & 1.8 & 21.0 & 0.6 & 0.01 & 0.2 & 15 & 0.13 & --- & --- & --- & 170 & --- & --- & -- & -- & --- & 3.30 & --- \\
\hline ER-EC-6 & --- & 10-Jun-03 & 119.6 & 1.8 & 4.6 & 0.2 & 0.10 & 0.2 & 27.4 & $<0.04$ & $<6$ & $<20$ & $<24$ & 4.1 & $<3$ & --- & --- & 2 & $<14$ & 5.43 & --- \\
\hline ER-EC-6 & --- & 10-Feb-00 & 128.0 & 2.0 & 4.0 & 0.0 & 0.15 & $<0.4$ & 22 & 0.42 & --- & -- & -- & 14 & -- & --- & --- & -- & --- & 4.72 & --- \\
\hline ER-EC-7 & --- & 21-Jul-03 & 28.0 & 2.0 & 20.2 & 2.2 & 0.02 & $<0.05$ & 20.7 & $<0.04$ & $<6$ & $<20$ & $<24$ & 81 & 2.4 & --- & --- & 4 & $<14$ & 1.86 & --- \\
\hline ER-EC-7 & -- & 5-Jun-00 & 35.0 & 2.8 & 22.0 & 2.0 & 0.04 & 0.4 & 13 & 0.23 & --- & --- & --- & 160 & --- & -- & --- & --- & --- & 2.10 & --- \\
\hline ER-EC-8 & --- & 1-Jul-03 & 120.4 & 4.9 & 10.1 & 0.3 & 0.15 & $<0.05$ & 28.4 & $<0.04$ & $<6$ & $<20$ & $<24$ & 2.2 & 10 & --- & --- & 2 & $<14$ & 4.81 & --- \\
\hline ER-EC-8 & --- & 12-Jul-00 & 120.0 & 5.6 & 11.0 & 0.5 & 0.15 & 0.2 & 22 & 0.11 & --- & --- & --- & 41 & --- & --- & --- & --- & --- & 4.80 & --- \\
\hline ER-18-2 & --- & 17-Jun-03 & 344.0 & 2.1 & 5.9 & 0.5 & 0.22 & 0.3 & 26.1 & $<0.04$ & 25 & 42 & 27 & 212 & 7.8 & --- & --- & 15 & $<14$ & 8.53 & --- \\
\hline ER-18-2 & --- & 21-Mar-00 & 365.0 & 1.8 & 6.1 & 0.2 & 0.28 & $<0.4$ & 22 & 0.06 & --- & --- & --- & 230 & -- & --- & --- & --- & --- & 7.26 & --- \\
\hline
\end{tabular}


Table 2.4 Environmental well trace metal data

\begin{tabular}{|c|c|c|c|c|c|c|c|c|c|c|c|c|c|c|c|c|}
\hline Well name & Test & $\begin{array}{c}\text { Sample } \\
\text { date }\end{array}$ & v & $\mathrm{Cr}$ & Co & $\mathrm{Ni}$ & $\mathrm{Cu}$ & $\mathrm{Zn}$ & $\mathrm{Br}$ * & $\mathbf{R b}$ & $\mathrm{Nb}$ & Ru & Cd & Sn & Cs & Eu \\
\hline Unit & & & $(\mu g / L)$ & $(\mu \mathrm{g} / L)$ & $(\mu g / L)$ & $(\mu g / L)$ & $(\mu g / L)$ & $(\mu g / L)$ & $(\mu g / L)$ & $(\mu g / L)$ & $(\mu g / L)$ & $(\mu g / L)$ & $(\mu \mathrm{g} / \mathrm{L})$ & $(\mu g / L)$ & $(\mu g / L)$ & $(\mu g / L)$ \\
\hline \multicolumn{17}{|c|}{ Clean Wells - Frenchman Flat } \\
\hline Water Well 5a & -- & 14-Aug-00 & --- & -- & --- & --- & --- & --- & -- & -- & -- & --- & --- & --- & -- & --- \\
\hline Water Well 5c & --- & 07-Aug-00 & -- & --- & --- & --- & --- & --- & --- & --- & --- & --- & --- & --- & --- & --- \\
\hline Water Well 5b & -- & 07-Aug-00 & -- & --- & --- & --- & --- & -- & --- & -- & -- & --- & -- & --- & -- & -- \\
\hline ER-5-4 & -- & 05-Jul-01 & -- & --- & --- & -- & --- & --- & --- & --- & --- & --- & --- & --- & --- & --- \\
\hline ER-5-4 \#2 & --- & 21-Nov-02 & -- & --- & --- & --- & --- & --- & --- & --- & --- & --- & -- & --- & --- & --- \\
\hline UE-5c WW & --- & 08-Aug-00 & --- & --- & --- & --- & --- & --- & --- & --- & --- & --- & --- & --- & --- & --- \\
\hline UE-5 PW-3 & --- & 09-Aug-00 & --- & --- & --- & --- & --- & -- & --- & -- & -- & --- & --- & --- & -- & --- \\
\hline ER-5-3 & -- & 28-Mar-01 & --- & -- & -- & --- & --- & -- & -- & -- & -- & --- & --- & --- & -- & --- \\
\hline ER-5-3 \#2 & -- & 17-May-01 & --- & -- & --- & --- & -- & --- & -- & -- & -- & --- & --- & --- & -- & --- \\
\hline Water Well 4a & --- & 08-Aug-00 & --- & --- & --- & -- & -- & --- & -- & -- & -- & --- & --- & --- & --- & --- \\
\hline \multicolumn{17}{|c|}{ Clean Wells - Yucca Flat } \\
\hline ER-2-1 & -- & 03-Sep-03 & --- & --- & --- & --- & --- & -- & -- & -- & -- & --- & --- & --- & -- & --- \\
\hline ER-6-1 \#2 & --- & 16-Jan-03 & --- & --- & --- & --- & --- & --- & --- & --- & --- & --- & --- & --- & --- & --- \\
\hline ER-6-2 & --- & 04-Aug-04 & 4.1 & 0.08 & 10.8 & 21.7 & $\mathrm{BD}$ & 32.3 & --- & 41 & 0.04 & 0.001 & 0.01 & 0.01 & 7.75 & 0.01 \\
\hline ER-7-1 & -- & 17-Jul-03 & --- & -- & --- & --- & --- & --- & --- & --- & --- & -- & --- & --- & --- & --- \\
\hline \multicolumn{17}{|c|}{ Clean Wells - Rainier Mesa } \\
\hline ER-12-1 & --- & 08-Dec-04 & 0.6 & 0.10 & 0.41 & 15.2 & $\mathrm{BD}$ & 6.5 & -- & 7.7 & 0.01 & 0.002 & 0.01 & 0.03 & 0.17 & $\mathrm{BD}$ \\
\hline ER-12-2 & --- & 01-Apr-03 & --- & -- & --- & --- & --- & --- & --- & --- & --- & --- & --- & --- & --- & --- \\
\hline ER-12-3 & -- & 06-Jul-05 & 2.2 & $<0.24$ & $<0.27$ & $<2.9$ & $<0.9$ & 259 & -- & 7.3 & $<0.042$ & $<0.3$ & $<0.6$ & --- & $<0.99$ & $<0.012$ \\
\hline ER-12-4 & --- & 16-Aug-05 & 1.2 & 1.6 & $<0.36$ & 14.1 & 5.8 & 538 & --- & 10.3 & $<0.24$ & $<0.27$ & $<0.33$ & --- & $<0.9$ & $<0.006$ \\
\hline \multicolumn{17}{|c|}{ Clean Wells - Pahute Mesa-Oasis Valley } \\
\hline ER-EC-1 & --- & 3-Jun-03 & --- & --- & --- & --- & --- & --- & --- & --- & --- & --- & --- & --- & --- & --- \\
\hline ER-EC-1 & -- & 1-Feb-00 & --- & --- & --- & --- & -- & --- & --- & --- & --- & --- & --- & --- & -- & --- \\
\hline ER-EC-2A & --- & 8-Jul-03 & --- & --- & --- & --- & --- & --- & --- & --- & --- & --- & --- & --- & --- & --- \\
\hline ER-EC-2A & --- & 27-Jul-00 & --- & --- & --- & --- & --- & --- & --- & --- & --- & --- & --- & --- & --- & --- \\
\hline ER-EC-4 & -- & 24-Jun-03 & -- & --- & --- & --- & --- & -- & -- & -- & -- & -- & -- & -- & -- & --- \\
\hline ER-EC-4 & -- & 17-Aug-00 & -- & --- & -- & --- & --- & -- & -- & -- & --- & --- & -- & --- & -- & --- \\
\hline ER-EC-5 & -- & 15-Jul-03 & --- & --- & --- & --- & -- & -- & --- & --- & -- & --- & -- & --- & -- & --- \\
\hline ER-EC-5 & --- & 25-May-00 & --- & --- & --- & --- & --- & --- & --- & --- & --- & --- & --- & --- & --- & -- \\
\hline ER-EC-6 & --- & 10-Jun-03 & --- & --- & --- & --- & --- & --- & --- & --- & --- & --- & --- & --- & --- & --- \\
\hline ER-EC-6 & -- & $10-$ Feb-00 & -- & -- & --- & -- & --- & -- & -- & --- & --- & -- & -- & --- & --- & -- \\
\hline ER-EC-7 & --- & 21-Jul-03 & --- & -- & --- & -- & --- & -- & --- & --- & --- & -- & --- & -- & --- & --- \\
\hline ER-EC-7 & -- & 5-Jun-00 & -- & -- & --- & -- & -- & -- & -- & -- & -- & -- & -- & -- & -- & -- \\
\hline ER-EC-8 & --- & 1-Jul-03 & --- & -- & --- & -- & --- & --- & --- & --- & --- & --- & --- & -- & --- & --- \\
\hline ER-EC-8 & -- & 12-Jul-00 & -- & -- & --- & -- & -- & -- & -- & -- & -- & -- & -- & --- & -- & -- \\
\hline ER-18-2 & --- & 17-Jun-03 & --- & -- & --- & --- & --- & --- & --- & --- & --- & --- & --- & --- & --- & --- \\
\hline ER-18-2 & --- & 21-Mar-00 & --- & --- & --- & --- & --- & --- & --- & --- & --- & --- & --- & --- & --- & --- \\
\hline
\end{tabular}

* Bromide measured by ICP-MS 
Table 2.5 Environmental well stable isotope data

\begin{tabular}{|c|c|c|c|c|c|c|c|c|c|c|c|c|c|c|}
\hline \multirow[b]{2}{*}{ Well name } & \multirow[b]{2}{*}{ Test } & \multirow[b]{2}{*}{$\begin{array}{l}\text { Sample } \\
\text { date }\end{array}$} & & & & & & & & & & & & \\
\hline & & & $\delta D_{\text {smow }}$ & $\delta^{18} \mathrm{O}_{\text {smow }}$ & $\delta^{13} \mathrm{C}_{\mathrm{PDB}}$ & ${ }^{3} \mathrm{He}$ & ${ }^{4} \mathrm{He}$ & $\mathbf{R} / \mathbf{R}_{\mathrm{a}}$ & ${ }^{20} \mathrm{Ne}^{*}$ & ${ }^{40} \mathrm{Ar}^{*}$ & $\mathrm{Kr}$ & ${ }^{130} \mathrm{Xe}^{*}$ & ${ }^{87} \mathrm{Srl}{ }^{86} \mathrm{Sr}$ & $\delta^{87} \mathrm{Sr}$ \\
\hline Unit & & & $\%$ & $\%$ & $\%$ & atoms $/ g$ & atoms $/ g$ & $\begin{array}{c}{ }^{3} \mathrm{He} / \mathrm{A}^{A} \mathrm{He} \\
\text { sample/ } \\
\text { air }\end{array}$ & atoms $/ g$ & atoms $/ g$ & atoms $/ g$ & atoms $/ g$ & ratio & $\%$ \\
\hline \multicolumn{15}{|c|}{ Clean Wells - Frenchman Flat } \\
\hline Water Well $5 a$ & --- & 14-Aug-00 & -110 & -13.8 & -4.8 & $4.62 \mathrm{E}+06$ & $3.16 \mathrm{E}+12$ & 1.06 & $9.43 \mathrm{E}+12$ & $1.59 \mathrm{E}+16$ & --- & --- & --- & --- \\
\hline Water Well $5 \mathrm{c}$ & --- & 07-Aug-00 & -110.5 & -14.0 & -6.0 & $3.41 \mathrm{E}+06$ & $8.67 \mathrm{E}+12$ & 0.28 & $5.64 \mathrm{E}+12$ & $9.90 \mathrm{E}+15$ & --- & --- & --- & --- \\
\hline Water Well 5b & -- & 07-Aug-00 & -108 & -13.1 & -9.5 & $3.85 E+06$ & $3.90 \mathrm{E}+12$ & 0.71 & $5.52 \mathrm{E}+12$ & $9.04 \mathrm{E}+15$ & -- & -- & -- & --- \\
\hline ER-5-4 & --- & 05-Jul-01 & -109 & -13.6 & -4.6 & $4.10 \mathrm{E}+06$ & $5.45 \mathrm{E}+13$ & 0.054 & $1.00 \mathrm{E}+13$ & $1.09 \mathrm{E}+16$ & --- & $2.90 \mathrm{E}+11$ & 0.71056 & 1.92 \\
\hline ER-5-4 \#2 & --- & 21-Nov-02 & -101 & -13.3 & +0.2 & $1.65 E+06$ & $1.35 E+12$ & 0.89 & $4.98 \mathrm{E}+12$ & $8.25 E+15$ & $1.81 \mathrm{E}+12$ & $2.43 E+11$ & 0.70902 & -0.25 \\
\hline UE-5c WW & --- & 08-Aug-00 & -106.5 & -13.7 & -7.2 & $5.18 \mathrm{E}+06$ & $1.89 \mathrm{E}+13$ & 0.20 & $4.91 \mathrm{E}+12$ & $8.85 E+15$ & --- & --- & --- & --- \\
\hline UE-5 PW-3 & --- & 09-Aug-00 & -105.5 & -13.5 & -7.5 & $4.34 \mathrm{E}+06$ & $2.15 \mathrm{E}+12$ & 1.46 & $7.03 \mathrm{E}+12$ & $1.15 \mathrm{E}+16$ & -- & -- & --- & --- \\
\hline ER-5-3 & --- & 28-Mar-01 & -108.5 & -14.1 & -8.0 & $3.09 \mathrm{E}+06$ & $5.20 \mathrm{E}+12$ & 0.43 & $9.33 E+12$ & $9.87 \mathrm{E}+15$ & --- & --- & 0.71017 & 1.37 \\
\hline ER-5-3 \#2 & --- & 17-May-01 & -110 & -14.1 & -4.3 & $2.42 \mathrm{E}+07$ & $1.28 \mathrm{E}+13$ & 1.37 & $5.00 \mathrm{E}+12$ & $4.75 E+15$ & --- & $1.34 \mathrm{E}+11$ & 0.71538 & 8.71 \\
\hline Water Well 4a & -- & 08-Aug-00 & -101 & -12.8 & -8.4 & $2.42 \mathrm{E}+06$ & $3.16 \mathrm{E}+12$ & 0.55 & $4.65 \mathrm{E}+12$ & $8.02 E+15$ & --- & --- & -- & -- \\
\hline \multicolumn{15}{|c|}{ Clean Wells - Yucca Flat } \\
\hline ER-2-1 & --- & 03-Sep-03 & -109.5 & -14.2 & -12.1 & $7.36 \mathrm{E}+07$ & $3.76 \mathrm{E}+12$ & 14.2 & $7.65 \mathrm{E}+12$ & $9.66 \mathrm{E}+15$ & $2.02 \mathrm{E}+12$ & $2.62 \mathrm{E}+11$ & 0.71210 & 4.09 \\
\hline ER-6-1 \#2 & --- & 16-Jan-03 & -105 & -14.1 & -5.9 & -- & -- & --- & -- & -- & -- & -- & 0.71295 & 5.29 \\
\hline ER-6-2 & -- & 04-Aug-04 & -105 & -14.1 & -4.0 & $6.67 \mathrm{E}+06$ & $7.05 E+12$ & 0.68 & $3.68 \mathrm{E}+12$ & $5.13 \mathrm{E}+15$ & $1.19 \mathrm{E}+12$ & $6.97 \mathrm{E}+09$ & 0.71281 & 5.09 \\
\hline ER-7-1 & --- & 17-Jul-03 & -106 & -14.1 & -5.8 & $3.28 \mathrm{E}+07$ & $4.07 \mathrm{E}+13$ & 0.58 & $4.79 \mathrm{E}+12$ & $7.44 \mathrm{E}+15$ & $1.81 \mathrm{E}+12$ & $2.66 \mathrm{E}+11$ & 0.71306 & 5.44 \\
\hline \multicolumn{15}{|c|}{ Clean Wells - Rainier Mesa } \\
\hline ER-12-1 & -- & 08-Dec-04 & -94.5 & -12.6 & -10.7 & $4.24 \mathrm{E}+06$ & $6.84 \mathrm{E}+12$ & 0.45 & $1.12 \mathrm{E}+13$ & $1.28 \mathrm{E}+16$ & $2.67 \mathrm{E}+12$ & $3.65 E+11$ & 0.71230 & 4.37 \\
\hline ER-12-2 & --- & 01-Apr-03 & -101 & -13.5 & -4.9 & $3.51 \mathrm{E}+06$ & $9.24 \mathrm{E}+13$ & 0.03 & $8.08 \mathrm{E}+12$ & $1.03 E+16$ & $1.91 \mathrm{E}+12$ & $1.16 \mathrm{E}+11$ & 0.71662 & 10.46 \\
\hline ER-12-3 & -- & 06-Jul-05 & -106 & -14.5 & -5.4 & $3.24 \mathrm{E}+06$ & $1.79 \mathrm{E}+13$ & 0.13 & $7.30 \mathrm{E}+12$ & $9.69 \mathrm{E}+15$ & $2.14 \mathrm{E}+12$ & $1.16 \mathrm{E}+10$ & 0.71055 & 1.90 \\
\hline ER-12-4 & --- & 16-Aug-05 & -103 & -13.7 & -7.6 & $7.81 E+05$ & $1.08 \mathrm{E}+12$ & 0.52 & $4.48 \mathrm{E}+12$ & $7.63 E+15$ & $1.82 \mathrm{E}+12$ & $1.19 \mathrm{E}+10$ & 0.71065 & 2.04 \\
\hline \multicolumn{15}{|c|}{ Clean Wells - Pahute Mesa-Oasis Valley } \\
\hline ER-EC-1 & --- & 3-Jun-03 & -116 & -14.9 & -3.1 & $9.44 \mathrm{E}+06$ & $1.13 E+13$ & 0.60 & $7.22 \mathrm{E}+12$ & $7.69 E+15$ & $1.58 \mathrm{E}+12$ & $2.29 E+11$ & 0.71056 & 1.92 \\
\hline ER-EC-1 & -- & 1-Feb-00 & -116 & -14.8 & -4.0 & $9.03 E+06$ & $9.24 \mathrm{E}+12$ & 0.71 & $9.93 E+12$ & $9.11 \mathrm{E}+15$ & -- & $2.34 \mathrm{E}+11$ & 0.71023 & 1.45 \\
\hline ER-EC-2A & --- & 8-Jul-03 & -116.5 & -14.9 & -2.0 & $8.36 \mathrm{E}+06$ & $6.97 \mathrm{E}+12$ & 0.87 & $4.28 \mathrm{E}+12$ & $5.69 E+15$ & $1.21 \mathrm{E}+12$ & $1.93 \mathrm{E}+11$ & 0.70912 & -0.11 \\
\hline ER-EC-2A & -- & 27-Jul-00 & -116 & -14.9 & -1.5 & $1.03 \mathrm{E}+07$ & $7.92 \mathrm{E}+12$ & 0.94 & $1.07 E+13$ & $1.02 \mathrm{E}+16$ & --- & -- & 0.70939 & 0.26 \\
\hline
\end{tabular}


Table 2.5 Environmental well stable isotope data (continued)

\begin{tabular}{|c|c|c|c|c|c|c|c|c|c|c|c|c|c|c|}
\hline Well name & Test & $\begin{array}{c}\text { Sample } \\
\text { date }\end{array}$ & $\delta \mathrm{D}_{\text {smow }}$ & $\delta^{18} \mathrm{O}_{\text {sMow }}$ & $\delta^{13} \mathrm{C}_{\mathrm{PDB}}$ & ${ }^{3} \mathrm{He}$ & ${ }^{4} \mathrm{He}$ & $\mathbf{R} / \mathbf{R}_{\mathbf{a}}$ & ${ }^{20} \mathrm{Ne}^{*}$ & ${ }^{40} \mathrm{Ar}^{*}$ & $\mathrm{Kr}$ & ${ }^{130} \mathrm{Xe}^{*}$ & $\left.{ }^{87} \mathrm{Sr}\right|^{86} \mathrm{Sr}$ & $\delta^{87} \mathrm{Sr}$ \\
\hline Unit & & & $\%$ & $\%$ & $\%$ & atoms $/ g$ & atoms $/ g$ & $\begin{array}{c}{ }^{3} \mathrm{He}^{4} \mathrm{He} \\
\text { sample/ } \\
\text { air } \\
\end{array}$ & atoms $/ g$ & atoms $/ g$ & atoms $/ g$ & atoms $/ g$ & ratio & $\%$ \\
\hline \multicolumn{15}{|c|}{ Clean Wells - Pahute Mesa-Oasis Valley } \\
\hline ER-EC-4 & --- & 24-Jun-03 & -114 & -14.6 & -1.1 & $2.00 E+07$ & $1.41 \mathrm{E}+13$ & 1.02 & $6.79 \mathrm{E}+12$ & $7.16 \mathrm{E}+15$ & $1.42 \mathrm{E}+12$ & $1.97 \mathrm{E}+11$ & 0.71010 & 1.27 \\
\hline ER-EC-4 & --- & 17-Aug-00 & -115 & -14.6 & -1.5 & $1.83 E+07$ & $1.30 \mathrm{E}+13$ & 1.01 & $7.66 \mathrm{E}+12$ & $8.55 E+15$ & --- & --- & 0.70998 & 1.11 \\
\hline ER-EC-5 & --- & 15-Jul-03 & -113 & -14.9 & -2.8 & $9.77 \mathrm{E}+06$ & $6.07 \mathrm{E}+12$ & 1.16 & $5.23 E+12$ & $6.54 \mathrm{E}+15$ & $1.34 \mathrm{E}+12$ & $1.96 \mathrm{E}+11$ & 0.70916 & -0.06 \\
\hline ER-EC-5 & --- & 25-May-00 & -113 & -14.9 & -2.5 & $1.06 \mathrm{E}+07$ & $7.09 \mathrm{E}+12$ & 1.08 & $8.88 \mathrm{E}+12$ & 8.37E+15 & --- & $1.84 \mathrm{E}+11$ & 0.70912 & -0.11 \\
\hline ER-EC-6 & --- & 10-Jun-03 & -117 & -15.0 & -2.7 & $1.72 \mathrm{E}+07$ & $1.79 \mathrm{E}+13$ & 0.69 & $4.76 \mathrm{E}+12$ & $6.64 \mathrm{E}+15$ & $1.49 \mathrm{E}+12$ & $2.26 \mathrm{E}+11$ & 0.71038 & 1.66 \\
\hline ER-EC-6 & --- & $10-$ Feb-00 & -116 & -15.0 & -3.4 & $1.76 \mathrm{E}+07$ & $1.75 \mathrm{E}+13$ & 0.73 & $6.90 \mathrm{E}+12$ & $7.46 \mathrm{E}+15$ & --- & $2.29 \mathrm{E}+11$ & 0.70982 & 0.88 \\
\hline ER-EC-7 & --- & 21-Jul-03 & -98 & -13.2 & -5.5 & $6.81 \mathrm{E}+06$ & $5.87 \mathrm{E}+12$ & 0.84 & $5.39 \mathrm{E}+12$ & $7.42 \mathrm{E}+15$ & $1.59 \mathrm{E}+12$ & $2.22 \mathrm{E}+11$ & 0.70948 & 0.39 \\
\hline ER-EC-7 & --- & 5-Jun-00 & -98 & -13.2 & -6.3 & $8.81 \mathrm{E}+06$ & $7.46 \mathrm{E}+12$ & 0.86 & $7.48 \mathrm{E}+12$ & $8.20 \mathrm{E}+15$ & --- & $2.35 E+11$ & 0.70932 & 0.17 \\
\hline ER-EC-8 & --- & 1-Jul-03 & -115 & -14.9 & -0.9 & $5.18 \mathrm{E}+06$ & $3.81 \mathrm{E}+12$ & 0.98 & $4.68 \mathrm{E}+12$ & $5.64 \mathrm{E}+15$ & $1.13 E+12$ & $1.64 \mathrm{E}+11$ & 0.70922 & 0.03 \\
\hline ER-EC-8 & --- & 12-Jul-00 & -116 & -14.8 & -1.0 & $6.43 E+06$ & $3.69 \mathrm{E}+12$ & 1.26 & $5.16 \mathrm{E}+12$ & $6.46 \mathrm{E}+15$ & --- & --- & 0.70882 & -0.54 \\
\hline ER-18-2 & --- & 17-Jun-03 & -111 & -14.7 & -0.5 & $5.11 \mathrm{E}+08$ & $1.83 \mathrm{E}+14$ & 2.01 & $2.95 \mathrm{E}+12$ & $3.52 E+15$ & $6.94 E+11$ & $1.08 \mathrm{E}+11$ & 0.70877 & -0.61 \\
\hline ER-18-2 & --- & 21-Mar-00 & -112 & -14.7 & -0.7 & $6.81 \mathrm{E}+08$ & $1.70 \mathrm{E}+14$ & 2.89 & $3.83 \mathrm{E}+12$ & $4.22 \mathrm{E}+14$ & --- & --- & 0.70861 & -0.84 \\
\hline
\end{tabular}

* Inconsistencies have been identified in the noble gas data. This issue will be addressed in the FY06 HRMP report. 
Table 2.6 Environmental well radiochemical data

\begin{tabular}{|c|c|c|c|c|c|c|c|c|c|c|c|c|}
\hline Well name & Test & $\begin{array}{l}\text { Sample } \\
\text { date }\end{array}$ & ${ }^{3} \mathrm{H}$ & ${ }^{3} \mathrm{H}$ & ${ }^{14} \mathrm{C}$ & ${ }^{14} \mathrm{C}$ & ${ }^{36} \mathrm{Cl} / \mathrm{Cl}$ & ${ }^{36} \mathrm{Cl}$ & ${ }^{85} \mathrm{Kr}$ & ${ }^{99} \mathrm{Tc}$ & ${ }^{129} \mid I^{127} I$ & 129 \\
\hline Unit & & date & $(p C i / L)$ & $(p C i / L)$ & $(p m c)$ & $(p C i / L)$ & ratio & $(p C i / L)$ & (pCi/L) & $(p C i / L)$ & ratio & $(p C i / L)$ \\
\hline Half-life (a) & & collected & 12.32 & 12.32 & 5730 & 5730 & & $3.01 E+05$ & 10.73 & $2.13 E+05$ & & $1.57 E+07$ \\
\hline Ref. date & & in field & collect. & time zero & collect. & collect. & & collect. & collect. & collect. & & collect. \\
\hline \multicolumn{13}{|c|}{ Clean Wells - Frenchman Flat } \\
\hline Water Well 5a & --- & 14-Aug-00 & 1.5 & --- & $2.60 \mathrm{E}+00$ & $9.64 \mathrm{E}-03$ & 8.43E-13 & 3.1E-04 & --- & --- & --- & --- \\
\hline Water Well $5 c$ & --- & 07-Aug-00 & $<1.5$ & --- & $3.40 \mathrm{E}+00$ & $1.24 \mathrm{E}-02$ & --- & --- & --- & --- & --- & --- \\
\hline Water Well 5b & --- & 07-Aug-00 & $<1.5$ & --- & $1.31 \mathrm{E}+01$ & $2.68 \mathrm{E}-02$ & 7.83E-13 & $6.0 \mathrm{E}-04$ & --- & --- & --- & --- \\
\hline ER-5-4 & --- & 05-Jul-01 & 2.5 & --- & $1.50 \mathrm{E}+00$ & $5.72 \mathrm{E}-03$ & 3.94E-13 & $3.5 \mathrm{E}-04$ & --- & --- & --- & --- \\
\hline ER-5-4 \#2 & --- & 21-Nov-02 & 156.8 & --- & $1.00 \mathrm{E}+00$ & 4.69E-03 & $1.76 \mathrm{E}-13$ & $3.0 \mathrm{E}-04$ & --- & --- & --- & --- \\
\hline UE-5c WW & --- & 08-Aug-00 & $<1.5$ & --- & $6.50 \mathrm{E}+00$ & $1.39 \mathrm{E}-02$ & --- & --- & --- & --- & --- & --- \\
\hline UE-5 PW-3 & --- & 09-Aug-00 & $<1.5$ & --- & $1.60 \mathrm{E}+01$ & $2.94 \mathrm{E}-02$ & --- & --- & --- & --- & --- & --- \\
\hline ER-5-3 & --- & 28-Mar-01 & $<1.5$ & --- & $8.50 \mathrm{E}+00$ & $1.73 \mathrm{E}-02$ & $8.42 \mathrm{E}-13$ & 4.3E-04 & --- & --- & --- & --- \\
\hline ER-5-3 \#2 & --- & 17-May-01 & $<1.5$ & --- & $1.60 \mathrm{E}+00$ & $1.55 \mathrm{E}-02$ & $2.29 \mathrm{E}-13$ & $2.9 \mathrm{E}-04$ & --- & --- & --- & --- \\
\hline Water Well 4a & --- & 08-Aug-00 & $<1.5$ & --- & $1.83 \mathrm{E}+01$ & $3.45 \mathrm{E}-02$ & $6.47 \mathrm{E}-13$ & 4.4E-04 & --- & --- & --- & --- \\
\hline \multicolumn{13}{|c|}{ Clean Wells - Yucca Flat } \\
\hline ER-2-1 & --- & 03-Sep-03 & 227.7 & --- & $1.82 \mathrm{E}+01$ & 4.14E-02 & 7.19E-13 & $1.04 \mathrm{E}-04$ & --- & --- & $<5 \mathrm{E}-10$ & --- \\
\hline ER-6-1 \#2 & --- & 16-Jan-03 & $\leq 30.8$ & --- & $2.40 \mathrm{E}+00$ & 7.39E-03 & 4.33E-13 & $1.4 \mathrm{E}-04$ & --- & --- & --- & --- \\
\hline ER-6-2 & --- & 04-Aug-04 & 92.2 & --- & $1.56 \mathrm{E}+00$ & $8.12 \mathrm{E}-03$ & $2.00 \mathrm{E}-13$ & 1.6E-04 & --- & --- & --- & --- \\
\hline ER-7-1 & --- & 17-Jul-03 & 117.2 & --- & $5.30 \mathrm{E}+00$ & $1.56 \mathrm{E}-02$ & 3.77E-13 & 1.18E-04 & --- & --- & $<1 \mathrm{E}-09$ & --- \\
\hline \multicolumn{13}{|c|}{ Clean Wells - Rainier Mesa } \\
\hline ER-12-1 & --- & 08-Dec-04 & 3.2 & --- & $1.10 \mathrm{E}+01$ & $3.40 \mathrm{E}-02$ & $7.80 \mathrm{E}-13$ & 4.56E-04 & --- & --- & --- & --- \\
\hline ER-12-2 & --- & 01-Apr-03 & 4.3 & --- & $1.50 \mathrm{E}+00$ & $5.39 \mathrm{E}-03$ & $6.90 \mathrm{E}-13$ & $1.6 \mathrm{E}-04$ & --- & --- & --- & --- \\
\hline ER-12-3 & --- & 06-Jul-05 & 0.5 & --- & $2.95 \mathrm{E}+00$ & 4.43E-03 & $5.39 \mathrm{E}-13$ & $1.0 \mathrm{E}-04$ & --- & --- & --- & --- \\
\hline ER-12-4 & -- & 16-Aug-05 & 89.7 & --- & $6.86 \mathrm{E}+00$ & $6.93 \mathrm{E}-03$ & $5.56 \mathrm{E}-13$ & 1.7E-04 & --- & --- & --- & --- \\
\hline \multicolumn{13}{|c|}{ Clean Wells - Pahute Mesa-Oasis Valley } \\
\hline ER-EC-1 & --- & 3-Jun-03 & $\leq 174$ & --- & $7.20 \mathrm{E}+00$ & $1.28 \mathrm{E}-02$ & $5.14 \mathrm{E}-13$ & 1.64E-03 & --- & --- & --- & --- \\
\hline ER-EC-1 & --- & 1-Feb-00 & $<1.5$ & --- & $5.90 \mathrm{E}+00$ & $1.05 \mathrm{E}-02$ & $5.46 \mathrm{E}-13$ & 1.75E-03 & --- & --- & --- & --- \\
\hline ER-EC-2A & --- & 8-Jul-03 & $\leq 93$ & --- & $7.70 \mathrm{E}+00$ & 1.52E-02 & $5.02 \mathrm{E}-13$ & $9.15 \mathrm{E}-04$ & --- & --- & --- & --- \\
\hline ER-EC-2A & --- & 27-Jul-00 & $<1.5$ & --- & $7.70 \mathrm{E}+00$ & $1.58 \mathrm{E}-02$ & 5.33E-13 & 1.11E-03 & --- & --- & --- & --- \\
\hline ER-EC-4 & --- & 24-Jun-03 & $<1.5$ & --- & $5.90 \mathrm{E}+00$ & 1.07E-02 & $4.80 \mathrm{E}-13$ & $1.28 \mathrm{E}-03$ & --- & --- & --- & --- \\
\hline ER-EC-4 & --- & 17-Aug-00 & $<1.5$ & --- & $5.00 \mathrm{E}+00$ & $9.54 \mathrm{E}-03$ & $5.61 \mathrm{E}-13$ & 1.77E-03 & --- & --- & --- & --- \\
\hline ER-EC-5 & --- & 15-Jul-03 & $\leq 9.0$ & --- & $7.50 \mathrm{E}+00$ & 1.62E-02 & $5.61 \mathrm{E}-13$ & $2.95 \mathrm{E}-04$ & --- & --- & --- & --- \\
\hline ER-EC-5 & --- & 25-May-00 & $<1.5$ & --- & $6.30 \mathrm{E}+00$ & 1.33E-02 & $6.53 \mathrm{E}-13$ & $3.5 \mathrm{E}-04$ & --- & --- & --- & --- \\
\hline ER-EC-6 & --- & 10-Jun-03 & $\leq 64$ & --- & $6.60 \mathrm{E}+00$ & 1.17E-02 & $5.07 \mathrm{E}-13$ & 8.65E-04 & --- & --- & --- & --- \\
\hline ER-EC-6 & --- & $10-$ Feb-00 & $<1.5$ & --- & $5.40 \mathrm{E}+00$ & $9.86 \mathrm{E}-03$ & $5.41 \mathrm{E}-13$ & 7.9E-04 & --- & --- & --- & --- \\
\hline ER-EC-7 & -- & 21-Jul-03 & 1.6 & --- & $4.62 \mathrm{E}+01$ & 4.80E-02 & 7.55E-13 & $9.47 \mathrm{E}-05$ & --- & --- & --- & --- \\
\hline ER-EC-7 & --- & 5-Jun-00 & $<1.5$ & --- & $3.65 \mathrm{E}+01$ & $5.22 \mathrm{E}-02$ & 1.18E-12 & $2.0 \mathrm{E}-04$ & --- & --- & --- & --- \\
\hline ER-EC-8 & --- & 1-Jul-03 & 5.4 & --- & $8.00 \mathrm{E}+00$ & 1.68E-02 & $4.90 \mathrm{E}-13$ & 7.65E-04 & --- & --- & --- & --- \\
\hline ER-EC-8 & --- & 12-Jul-00 & $<1.5$ & --- & $8.70 \mathrm{E}+00$ & $1.80 \mathrm{E}-02$ & 4.63E-13 & $8.8 \mathrm{E}-04$ & --- & --- & --- & --- \\
\hline ER-18-2 & --- & 17-Jun-03 & $<1.5$ & --- & 4.00E-01 & 4.13E-03 & $2.31 \mathrm{E}-13$ & $9.38 \mathrm{E}-05$ & --- & --- & --- & --- \\
\hline ER-18-2 & --- & 21-Mar-00 & $<1.5$ & --- & $1.60 \mathrm{E}+00$ & 1.70E-02 & 3.02E-13 & 1.3E-04 & --- & --- & --- & --- \\
\hline
\end{tabular}


Table 2.7 Environmental well radiochemical data

\begin{tabular}{|c|c|c|c|c|c|c|c|c|c|c|}
\hline Well name & Test & $\begin{array}{c}\text { Sample } \\
\text { date }\end{array}$ & ${ }^{234} \mathrm{U} I^{238} \mathrm{U}$ & $\begin{array}{c}{ }^{234} \mathrm{U} I^{238} \mathrm{U} \\
\text { activity } \\
\text { ratio }\end{array}$ & ${ }^{234} \mathrm{U} I^{235} \mathrm{U}$ & ${ }^{235} \mathrm{U} I^{238} \mathrm{U}$ & ${ }^{234} U$ & ${ }^{235} U$ & ${ }^{238} U$ & ${ }^{239,240} \mathrm{Pu}$ \\
\hline $\begin{array}{c}\text { Unit } \\
\text { Half-life (a) } \\
\text { Ref. date }\end{array}$ & & $\begin{array}{c}\text { date } \\
\text { collected } \\
\text { in field }\end{array}$ & ratio & ratio & ratio & ratio & $\begin{array}{c}\text { (pCi/L) } \\
2.46 E+05 \\
\text { collect. }\end{array}$ & $\begin{array}{c}\text { (pCi/L) } \\
7.04 E+08 \\
\text { collect. }\end{array}$ & $\begin{array}{c}\text { (pCi/L) } \\
4.47 E+09 \\
\text { collect. }\end{array}$ & $\begin{array}{l}\text { (pCi/L) } \\
\text { collect. }\end{array}$ \\
\hline \multicolumn{11}{|c|}{ Clean Wells - Frenchman Flat } \\
\hline Water Well $5 a$ & --- & 14-Aug-00 & -- & -- & -- & -- & --- & -- & -- & -- \\
\hline Water Well 5c & --- & 07-Aug-00 & --- & --- & --- & --- & --- & --- & --- & --- \\
\hline Water Well 5b & --- & 07-Aug-00 & --- & --- & --- & --- & --- & --- & --- & --- \\
\hline ER-5-4 & --- & 05-Jul-01 & 7.46E-05 & 1.36 & 1.03E-02 & $7.25 \mathrm{E}-03$ & 7.30E-01 & $2.50 \mathrm{E}-02$ & $5.30 \mathrm{E}-01$ & --- \\
\hline ER-5-4 \#2 & --- & 21-Nov-02 & 7.19E-05 & 1.31 & 9.92E-03 & $7.25 \mathrm{E}-03$ & $1.61 \mathrm{E}+01$ & 5.70E-01 & $1.22 \mathrm{E}+01$ & --- \\
\hline UE-5c WW & -- & 08-Aug-00 & --- & -- & -- & -- & --- & -- & -- & -- \\
\hline UE-5 PW-3 & --- & 09-Aug-00 & --- & --- & --- & --- & --- & --- & --- & --- \\
\hline ER-5-3 & --- & 28-Mar-01 & $1.86 \mathrm{E}-04$ & 3.40 & $2.57 \mathrm{E}-02$ & 7.26E-03 & $1.12 \mathrm{E}+00$ & $1.50 \mathrm{E}-02$ & $3.30 \mathrm{E}-01$ & --- \\
\hline ER-5-3 \#2 & --- & 17-May-01 & $1.80 \mathrm{E}-04$ & 3.29 & $2.48 \mathrm{E}-02$ & $7.26 \mathrm{E}-03$ & $5.60 \mathrm{E}-01$ & 8.00E-03 & $1.70 \mathrm{E}-01$ & --- \\
\hline Water Well 4a & -- & 08-Aug-00 & --- & --- & --- & --- & --- & --- & --- & -- \\
\hline \multicolumn{11}{|c|}{ Clean Wells - Yucca Flat } \\
\hline ER-2-1 & --- & 03-Sep-03 & $1.48 \mathrm{E}-04$ & 2.71 & $2.04 \mathrm{E}-02$ & $7.25 \mathrm{E}-03$ & $3.11 \mathrm{E}+00$ & $5.30 \mathrm{E}-02$ & $1.14 \mathrm{E}+00$ & --- \\
\hline ER-6-1 \#2 & -- & 16-Jan-03 & $2.30 \mathrm{E}-04$ & 4.19 & 3.17E-02 & $7.25 \mathrm{E}-03$ & $4.55 \mathrm{E}+00$ & $5.00 \mathrm{E}-02$ & $1.07 \mathrm{E}+00$ & --- \\
\hline ER-6-2 & --- & 04-Aug-04 & $2.39 \mathrm{E}-04$ & 4.35 & $3.30 \mathrm{E}-02$ & $7.25 \mathrm{E}-03$ & $3.20 \mathrm{E}+00$ & $3.40 \mathrm{E}-02$ & $7.24 \mathrm{E}-01$ & --- \\
\hline ER-7-1 & --- & 17-Jul-03 & $1.88 \mathrm{E}-04$ & 3.43 & $2.59 \mathrm{E}-02$ & $7.25 \mathrm{E}-03$ & $1.33 \mathrm{E}+00$ & $1.80 \mathrm{E}-02$ & $3.83 E-01$ & --- \\
\hline \multicolumn{11}{|c|}{ Clean Wells - Rainier Mesa } \\
\hline ER-12-1 & --- & 08-Dec-04 & 3.79E-04 & 1.62 & $5.22 \mathrm{E}-02$ & $7.25 \mathrm{E}-03$ & $1.43 E+00$ & 4.21E-02 & 8.68E-01 & --- \\
\hline ER-12-2 & -- & 01-Apr-03 & 1.79E-04 & 3.26 & $2.45 \mathrm{E}-02$ & $7.30 \mathrm{E}-03$ & $1.90 \mathrm{E}-02$ & $2.70 \mathrm{E}-04$ & $5.70 \mathrm{E}-03$ & -- \\
\hline ER-12-3 & -- & 06-Jul-05 & 1.15E-04 & 2.09 & $1.58 \mathrm{E}-02$ & $7.26 \mathrm{E}-03$ & $1.30 \mathrm{E}+00$ & $2.90 \mathrm{E}-02$ & $6.12 \mathrm{E}-01$ & -- \\
\hline ER-12-4 & -- & 16-Aug-05 & $1.06 \mathrm{E}-04$ & 1.93 & 1.46E-02 & $7.24 \mathrm{E}-03$ & $1.92 \mathrm{E}-01$ & $5.00 \mathrm{E}-03$ & $9.90 \mathrm{E}-02$ & --- \\
\hline \multicolumn{11}{|c|}{ Clean Wells - Pahute Mesa-Oasis Valley } \\
\hline ER-EC-1 & --- & 3-Jun-03 & $1.94 \mathrm{E}-04$ & 3.54 & 2.66E-02 & 7.27E-03 & $1.13 \mathrm{E}+01$ & $1.48 \mathrm{E}-01$ & $3.17 \mathrm{E}+00$ & --- \\
\hline ER-EC-1 & --- & $1-F e b-00$ & $2.10 \mathrm{E}-04$ & 3.82 & $2.89 \mathrm{E}-02$ & $7.25 \mathrm{E}-03$ & $1.05 \mathrm{E}+01$ & $1.26 \mathrm{E}-01$ & $2.71 \mathrm{E}+00$ & -- \\
\hline ER-EC-2A & --- & 8-Jul-03 & $2.74 \mathrm{E}-04$ & 5.01 & 3.78E-02 & $7.25 \mathrm{E}-03$ & $1.19 \mathrm{E}+01$ & 1.10E-01 & $2.35 E+00$ & --- \\
\hline ER-EC-2A & --- & 27-Jul-00 & $2.25 \mathrm{E}-04$ & 4.11 & 3.09E-02 & 7.27E-03 & $1.28 \mathrm{E}+01$ & $1.44 \mathrm{E}-01$ & $3.08 \mathrm{E}+00$ & --- \\
\hline ER-EC-4 & --- & 24-Jun-03 & 1.59E-04 & 2.90 & 2.19E-02 & $7.27 \mathrm{E}-03$ & $3.99 \mathrm{E}+00$ & $6.40 \mathrm{E}-02$ & $1.36 \mathrm{E}+00$ & --- \\
\hline ER-EC-4 & --- & 17-Aug-00 & $1.59 \mathrm{E}-04$ & 2.90 & $2.18 \mathrm{E}-02$ & $7.29 \mathrm{E}-03$ & $3.97 \mathrm{E}+00$ & $6.40 \mathrm{E}-02$ & $1.39 \mathrm{E}+00$ & --- \\
\hline ER-EC-5 & -- & 15-Jul-03 & 3.53E-04 & 6.45 & 4.85E-02 & 7.27E-03 & $7.27 \mathrm{E}+00$ & $5.20 \mathrm{E}-02$ & $1.12 \mathrm{E}+00$ & --- \\
\hline ER-EC-5 & --- & 25-May-00 & 3.51E-04 & 6.41 & 4.82E-02 & $7.28 \mathrm{E}-03$ & $7.20 \mathrm{E}+00$ & $5.20 \mathrm{E}-02$ & $1.11 \mathrm{E}+00$ & --- \\
\hline ER-EC-6 & --- & 10-Jun-03 & 2.03E-04 & 3.71 & $2.80 \mathrm{E}-02$ & 7.27E-03 & $6.79 \mathrm{E}+00$ & 8.50E-02 & $1.81 \mathrm{E}+00$ & -- \\
\hline ER-EC-6 & -- & $10-F e b-00$ & 2.23E-04 & 4.07 & 3.07E-02 & 7.27E-03 & $6.52 \mathrm{E}+00$ & 7.40E-02 & $1.58 \mathrm{E}+00$ & -- \\
\hline ER-EC-7 & --- & 21-Jul-03 & 3.97E-04 & 7.26 & $5.48 \mathrm{E}-02$ & $7.25 \mathrm{E}-03$ & $4.57 \mathrm{E}+00$ & 2.90E-02 & $6.22 \mathrm{E}-01$ & --- \\
\hline ER-EC-7 & --- & 5-Jun-00 & 3.97E-04 & 7.26 & $5.46 \mathrm{E}-02$ & $7.28 \mathrm{E}-03$ & $5.19 \mathrm{E}+00$ & 3.30E-02 & 7.10E-01 & --- \\
\hline ER-EC-8 & --- & 1-Jul-03 & 2.77E-04 & 5.06 & 3.82E-02 & $7.24 \mathrm{E}-03$ & $8.22 \mathrm{E}+00$ & 7.50E-02 & $1.61 \mathrm{E}+00$ & --- \\
\hline ER-EC-8 & --- & 12-Jul-00 & $2.78 \mathrm{E}-04$ & 5.08 & 3.82E-02 & $7.28 \mathrm{E}-03$ & $8.18 \mathrm{E}+00$ & $7.40 \mathrm{E}-02$ & $1.59 \mathrm{E}+00$ & --- \\
\hline ER-18-2 & --- & 17-Jun-03 & 6.98E-04 & 12.76 & 9.59E-02 & $7.29 \mathrm{E}-03$ & $3.67 \mathrm{E}+01$ & 1.33E-01 & $2.85 \mathrm{E}+00$ & --- \\
\hline ER-18-2 & --- & 21-Mar-00 & 6.95E-04 & 12.70 & $9.56 \mathrm{E}-02$ & 7.27E-03 & $3.54 \mathrm{E}+01$ & 1.29E-01 & $2.76 \mathrm{E}+00$ & --- \\
\hline
\end{tabular}




\title{
Chapter 3
}

\section{Distribution of ${ }^{99} \mathrm{Tc}$ and ${ }^{129} \mathrm{I}$ in the Vicinity of Underground Nuclear Tests}

\author{
P. Zhao ${ }^{1}$, Q. (Max) $\mathrm{Hu}^{1}$, T.P. Rose ${ }^{1}$, G.J. Nimz ${ }^{2}$, and M. Zavarin ${ }^{1}$ \\ ${ }^{1}$ Chemical Biology and Nuclear Science Division \\ ${ }^{2}$ Energy and Environment Directorate \\ Lawrence Livermore National Laboratory
}

\subsection{Introduction}

${ }^{99} \mathrm{Tc}$ and ${ }^{129} \mathrm{I}$ are actinide fission products with long half-lives $\left(2.15 \times 10^{5}\right.$ years and $1.57 \times 10^{7}$ years, respectively), both of which occur as high-solubility anionic species in groundwater. Due to their weak sorption characteristics, both ${ }^{99} \mathrm{Tc}$ and ${ }^{129} \mathrm{I}$ have a high potential for migration in the subsurface environment.

At underground nuclear testing sites, the migration of ${ }^{99} \mathrm{Tc}$ and ${ }^{129} \mathrm{I}$ in groundwater is influenced by the initial distribution of these radionuclides in nuclear test debris. Unfortunately, very little information is available on this initial distribution. Our current understanding of ${ }^{99} \mathrm{Tc}$ and ${ }^{129} \mathrm{I}$ distribution within the test cavity-chimney is based primarily on IAEA experience from French underground tests in French Polynesia (IAEA, 1998). Based on both physical and chemical characteristics, the fraction of ${ }^{99} \mathrm{Tc}$ and ${ }^{129} \mathrm{I}$ incorporated into melt glass from an underground nuclear test was estimated to be $80 \%$ and $50 \%$, respectively.

The main objectives of this investigation were to: (1) measure the ${ }^{99} \mathrm{Tc}$ and ${ }^{129} \mathrm{I}$ concentrations in melt glass and rubble samples from an underground test cavity, and use the results to empirically assess the IAEA partitioning estimates; (2) measure ${ }^{99} \mathrm{Tc}$ and ${ }^{129} \mathrm{I}$ in groundwater samples from a number of NTS near-field wells; and (3) compare groundwater and test debris data to determine water-rock partitioning $\left(\mathrm{K}_{\mathrm{d}}\right)$.

\subsection{Experimental}

\subsubsection{Technetium Analyses}

For groundwater samples, we used TEVA resin columns to enrich and separate ${ }^{99} \mathrm{Tc}$ from interfering elements (Einchrom, 2002). Depending on the ${ }^{99} \mathrm{Tc}$ concentration, sample volumes of $10 \mathrm{~mL}$ to 2 liters were used. For melt glass and rock samples, a fusion method (Dixon et al., 1997; ASTM, 2005) was used wherein a powdered sample was reacted with sodium peroxide and low carbonate sodium hydroxide at $700{ }^{\circ} \mathrm{C}$ for 30 minutes. This method is effective in fusing all rock-forming minerals while minimizing Tc 
volatilization (and potential loss) during the digestion process. The fusion cake was dissolved in nitric acid and a TEVA column was used to separate and purify the ${ }^{99} \mathrm{Tc}$. A modified isotope dilution ICP-MS method was used for ${ }^{99} \mathrm{Tc}$ analyses. The NIST SRM-4288A solid and an aqueous solution of $32.61 \pm 0.37 \mathrm{kBq} / \mathrm{g}{ }^{99} \mathrm{Tc}(\mathrm{VII})$ in $1 \times 10^{-3} \mathrm{M}$ $\mathrm{KOH}$ were used as ${ }^{99} \mathrm{Tc}$ reference standards. Stable rhenium was spiked in each sample (both groundwater and solid) as a tracer to monitor rhenium and, by analogy, ${ }^{99} \mathrm{Tc}$ recovery.

\subsubsection{Iodine Analyses}

NTS groundwater samples are normally in the neutral to slightly alkaline $\mathrm{pH}$ range and were directly analyzed for stable iodine after spiking with $5 \mu \mathrm{g} / \mathrm{L}$ of internal standard (rhodium, Rh) in 1\% CFA-C. The CFA-C is a surfactant that coats the glassware and reduces the memory effect of iodine in the ICP-MS introduction system (Mas et al., 2004). Groundwater samples were prepared for ${ }^{129} \mathrm{I}$ analysis on the Accelerator Mass Spectrometer (AMS) using standard methods reported in the literature (Moran et al., 1995).

A microwave sample preparation system was used to digest and extract iodine from melt glass and rock samples. $4 \mathrm{~mL}$ of $5 \%$ tetra-methyl ammonium hydroxide (TMAH) solution was added to $0.5-1 \mathrm{~g}$ of powdered sample in a Teflon vessel for the microwave digestion (Uchida et a., 1992; Zhou et al., 1996; Hamada et al., 1999). The power level and time for each digestion stage were programmed to reach a maximum value of $800 \mathrm{~W}$ over 15 minutes, and held there for an additional 10 minutes. To minimize potential iodine loss, the maximum temperature and pressure was not allowed to exceed $90{ }^{\circ} \mathrm{C}$ and $1241 \mathrm{kPa}$, respectively. After digestion, $6 \mathrm{~mL}$ of $1 \%$ CFA-C was added to the sample vessel. The total iodine in the supernatant was analyzed directly by ICP-MS. The ${ }^{129} \mathrm{I}$ targets were made using a stable ${ }^{127} \mathrm{I}$ carrier containing low ${ }^{129} \mathrm{I}$ to form AgI solid (Marchetti et al., 1997); the ${ }^{129} \mathrm{I} /{ }^{127} \mathrm{I}$ ratio was measured by AMS.

\subsection{Results and Discussion}

\subsubsection{Results of Groundwater Samples}

A total of 24 groundwater samples from 13 sampling sites were analyzed for ${ }^{99} \mathrm{Tc}$ and ${ }^{129} \mathrm{I}$. Table 3.1 presents the measured ${ }^{99} \mathrm{Tc}$ concentrations in NTS groundwater samples using the ICP-MS method. For comparison, data from independent AMS (Bergquist et al., 2000) and liquid scintillation counting (LSC; this study) measurements of the same samples are also included. Measurement uncertainties reported in Table 3.1 are calculated from replicate analyses. The instrumental uncertainties for ${ }^{99} \mathrm{Tc}$ and ${ }^{129} \mathrm{I}$ analyses are $<2 \%$ by ICP-MS measurements and $2-4 \%$ for ${ }^{129} \mathrm{I} /{ }^{127} \mathrm{I}$ ratio measurements by AMS. However, error propagation in overall procedures for groundwater samples results in uncertainties of 5 to $10 \%$. Table 3.1 also includes an Irish Sea Water sample as a reference standard (Bergquist et al., 2000). Our measured ${ }^{99} \mathrm{Tc}$ value for the reference standard is in good agreement with the accepted value $(0.22 \pm 0.01 \mathrm{~Bq} / \mathrm{L})$. 
Table 3.1 Technetium and Iodine in NTS Groundwater Samples

\begin{tabular}{|c|c|c|c|c|c|c|c|}
\hline Test Name & Sampling Well / Location & Sampling Date & $\begin{array}{c}{ }^{99} \mathrm{Tc} \text { (ICP-MS) } \\
\text { (Bq/L) }\end{array}$ & $\begin{array}{c}{ }^{99} \mathrm{Tc} \text { (other methods) } \\
(\mathrm{Bq} / \mathrm{L})\end{array}$ & $\begin{array}{c}\text { Total lodine } \\
(\mu \mathrm{g} / \mathrm{L})\end{array}$ & ${ }^{129} I I{ }^{127} I$ & $\begin{array}{c}{ }^{129} \mathrm{I} \\
(\mathrm{mBq} / \mathrm{L}) \\
\end{array}$ \\
\hline ALEMAN & UE-3e \#4; $58 \mathrm{~m}$ from working point & 09/23/1998 & $0.32 \pm 0.01$ & $0.48 \pm 0.01^{a}$ & 11.3 & n.a. & \\
\hline \multirow{2}{*}{ ALMENDRO } & \multirow{2}{*}{ U-19v PS1ds; Cavity/chimney } & 08/18/1999 & $2.8 \mathrm{E}-03$ & n.a. & 7.4 & $1.06 \mathrm{E}-03$ & 53 \\
\hline & & $07 / 23 / 2003$ & 4.1E-04 & n.a. & 10.1 & $1.41 \mathrm{E}-03$ & 95 \\
\hline \multirow{2}{*}{ BILBY } & \multirow{2}{*}{ U-3cn PS2; Chimney } & $12 / 18 / 2001$ & $3.06 \pm 0.03$ & $2.96^{b}$ & 9.1 & $9.74 \mathrm{E}-05$ & 5.9 \\
\hline & & $12 / 09 / 2004$ & $2.32 \pm 0.23$ & $2.50 \pm 0.07^{b}$ & 9.5 & $1.48 \mathrm{E}-04$ & 9.4 \\
\hline BOURBON & UE-7nS; 137 m from working point & $08 / 21 / 2001$ & $<0.04$ & n.a. & 23.1 & $1.47 \mathrm{E}-07$ & 0.023 \\
\hline \multirow{5}{*}{ CAMBRIC } & RNM-1; Cavity & 06/03/2004 & $<1.3 \mathrm{E}-4$ & $<0.037^{b}$ & 16.2 & 2.06E-07 & 0.022 \\
\hline & \multirow{2}{*}{ RNM-2S; $91 \mathrm{~m}$ from working point } & 10/11/1999 & $<1.3 \mathrm{E}-5$ & $(4.8 \pm 2.6) \times 1 \mathrm{E}-5^{\mathrm{a}}$ & 11 & $2.12 \mathrm{E}-07$ & 0.016 \\
\hline & & $07 / 10 / 2003$ & $<1.3 \mathrm{E}-6$ & n.a. & 12.2 & $6.16 \mathrm{E}-07$ & 0.050 \\
\hline & \multirow{2}{*}{ UE-5n; $510 \mathrm{~m}$ from RNM-2S } & 09/09/1999 & 1.7E-04 & $(8.5 \pm 7.0) \times 1 E-5^{a}$ & 30 & 3.51E-09 & 0.0007 \\
\hline & & $02 / 12 / 2004$ & 2.7E-04 & n.a. & 19 & $5.02 \mathrm{E}-07$ & 0.064 \\
\hline \multirow{2}{*}{ CAMEMBERT } & \multirow{2}{*}{ U-19q PS1d; Chimney } & $10 / 21 / 1998$ & n.a. & $(3.1 \pm 0.7) \times 1 E-3^{a}$ & 4.1 & $5.54 \mathrm{E}-06$ & 0.15 \\
\hline & & $07 / 16 / 2003$ & 4.8E-04 & n.a. & 4 & $2.77 \mathrm{E}-06$ & 0.074 \\
\hline \multirow{3}{*}{ CHESHIRE } & \multirow{3}{*}{ U-20n PS1ddh; Cavity } & $07 / 25 / 1998$ & n.a. & $1.15 \pm 0.25^{a}$ & 5.9 & n.a. & n.a. \\
\hline & & 09/21/1998 & $0.80 \pm 0.04$ & $0.74 \pm 0.02^{a}, 0.422^{b}$ & 5.8 & n.a. & n.a. \\
\hline & & $10 / 12 / 1999$ & $0.57 \pm 0.04$ & $0.83 \pm 0.16^{a}$ & 13 & 4.91E-05 & 4.3 \\
\hline CHANCELLOR & U-19ad PS1A; Cavity & $09 / 27 / 2004$ & $1.70 \pm 0.01$ & n.a. & 19.4 & 5.59E-04 & 73 \\
\hline \multirow{2}{*}{ DALHART } & \multirow{2}{*}{ U-4u PS2a; Chimney } & 08/16/1999 & $0.49 \pm 0.02$ & $0.59 \pm 0.02^{a}, 0.55^{b}$ & 5.8 & $2.80 \mathrm{E}-05$ & 1.1 \\
\hline & & $10 / 09 / 2003$ & $1.30 \pm 0.13$ & $1.29 \pm 0.07^{b}$ & 7.1 & $1.04 \mathrm{E}-04$ & 4.9 \\
\hline NASH & UE-2ce; 183 m from working point & $08 / 22 / 2001$ & $<1.3 \mathrm{E}-4$ & n.a. & 9.4 & 1.88E-05 & 1.2 \\
\hline \multirow{4}{*}{ TYBO /BENHAM $^{\mathrm{e}}$} & \multirow{2}{*}{$\begin{array}{l}\text { ER-20-5 \#1 }(701-784 \mathrm{~m})^{\mathrm{d}} ; 280 / 1300 \mathrm{~m} \\
\text { from TYBO /BENHAM working points }\end{array}$} & 07/09/1998 & 0.022 & $0.010 \pm 0.002^{a}$ & 7 & $2.14 \mathrm{E}-04$ & 10.0 \\
\hline & & $11 / 30 / 2004$ & 0.017 & $<0.04^{b}$ & 5.4 & 1.99E-04 & 7.2 \\
\hline & \multirow{2}{*}{$\begin{array}{l}\text { ER-20-5 \#3 (1046-1183 m) } ; 280 / 1300 \mathrm{~m} \\
\text { from TYBO /BENHAM working points }\end{array}$} & 04/30/1998 & $<0.04$ & $<0.001^{a}$ & 8.2 & n.a. & n.a. \\
\hline & & $11 / 29 / 2004$ & 7.0E-04 & n.a. & 4.7 & $1.66 \mathrm{E}-06$ & 0.052 \\
\hline Irish Sea Water & IAEA-381 & & 0.2 & $0.22 \pm 0.01^{c}$ & & & \\
\hline
\end{tabular}

n.a. $=$ not analyzed.

${ }^{\mathrm{a}}={ }^{99} \mathrm{TC}$ analyses performed by LSC; ${ }^{\mathrm{b}}={ }^{99} \mathrm{TC}$ analyses performed by AMS; ${ }^{\mathrm{c}}=$ Literature reported value; ${ }^{\mathrm{d}}=$ open interval where sampling occurred; ${ }^{\mathrm{e}}=\mathrm{Both}$ the $\mathrm{TYBO}$ and $\mathrm{BENHAM}$ tests are listed since the ER-20-5 well cluster was drilled in the near-field ( $\sim 300 \mathrm{~m}$ from the surface ground zero) environment of the TYBO test. However, Pu isotopic signatures indicate the radionuclides in water samples from ER-20-5 wells are derived from the BENHAM test detonated $~ 1300$ m up-gradient (Kersting et al., 1999). 
In general, cavity water samples contained higher concentrations of ${ }^{99} \mathrm{Tc}$ compared to near-field satellite wells. The highest values were observed in well U-3cn PS\#2a (the BILBY test), with $3.1 \mathrm{~Bq} / \mathrm{L}$ in 2001 and $2.3 \mathrm{~Bq} / \mathrm{L}$ in 2004 . Only three NTS near-field wells have produced water with ${ }^{99} \mathrm{Tc}$ activities above $1 \mathrm{~Bq} / \mathrm{L}$. Concentrations lower than 0.3 $\mathrm{mBq} / \mathrm{L}{ }^{99} \mathrm{Tc}$ were found in near-field satellite monitoring wells. Downstream migration of ${ }^{99} \mathrm{Tc}$ is apparent. However, local geochemical conditions, such as reducing conditions (sometimes encountered in test cavities) may be limiting ${ }^{99} \mathrm{Tc}$ migration from the test cavity.

Using Re to trace extraction efficiency, the ${ }^{99} \mathrm{Tc}$ concentration in U-19ad PS\#1A groundwater (CHANCELLOR test) was measured to be $1.70 \pm 0.01 \mathrm{~Bq} / \mathrm{L}$. This value was confirmed by an independent set of standard addition measurements. Using a TEVA column for pre-concentration and purification of 1-liter water samples, ICP-MS can provide a ${ }^{99} \mathrm{Tc}$ method detection limit (MDL) of $0.13 \mathrm{mBq} / \mathrm{L}$. This is about one order of magnitude higher than that achieved by AMS, which has a reported MDL of $0.01 \mathrm{mBq}$ or $1 \times 10^{8}$ atoms with $15-25 \%$ error for a 1-liter sample volume (Bergquist et al., 2000). The LSC detection limit is $\sim 37 \mathrm{mBq} / \mathrm{L}$.

The total stable ${ }^{127} \mathrm{I}$ concentrations, ${ }^{129} \mathrm{I} /{ }^{127} \mathrm{I}$ ratios, and corresponding ${ }^{129} \mathrm{I}$ activities in NTS groundwaters are also reported in Table 3.1. The ratio of ${ }^{129} \mathrm{I}^{127} \mathrm{I}$ in cavity and satellite well samples range from $10^{-3}$ to $10^{-6}$ and $10^{-4}$ to $10^{-9}$, respectively. The ${ }^{129} \mathrm{I} /{ }^{127} \mathrm{I}$ ratio of uncontaminated environmental samples is $\leq 10^{-13}$. The results suggest that ${ }^{129} \mathrm{I}$ is migrating at a rate similar to ${ }^{99} \mathrm{Tc}$.

\subsubsection{Results of Solid Samples}

Melt glass and rubble samples were recovered from the CHANCELLOR (U-19ad) underground test cavity during a drilling operation in 2004. Figure 3.1 shows the spatial distribution of samples collected from the test cavity. Samples were chosen over a wide depth interval ( $\sim 130$ meters). Table 3.2 lists the measured ${ }^{99} \mathrm{Tc}$, total iodine, and ${ }^{129} \mathrm{I} /{ }^{127} \mathrm{I}$ ratios in the test debris samples. The uncertainties in measurements of ${ }^{99} \mathrm{Tc}$ and ${ }^{129} \mathrm{I} /{ }^{127} \mathrm{I}$ ratios for the solid samples are similar to if not smaller than those for the water samples. We obtained $>95 \%$ chemical recovery of total iodine from three IAEA/SRM soil samples using the microwave digestion/TMAH extraction method. However, iodine recovery in melt glass samples may be lower due to the difference in sample matrix (soil vs. melt glass). We are currently assessing the quartz tube combustion method reported by Muramatsu and Wedepohl (1998) for total iodine measurement in rock samples. 


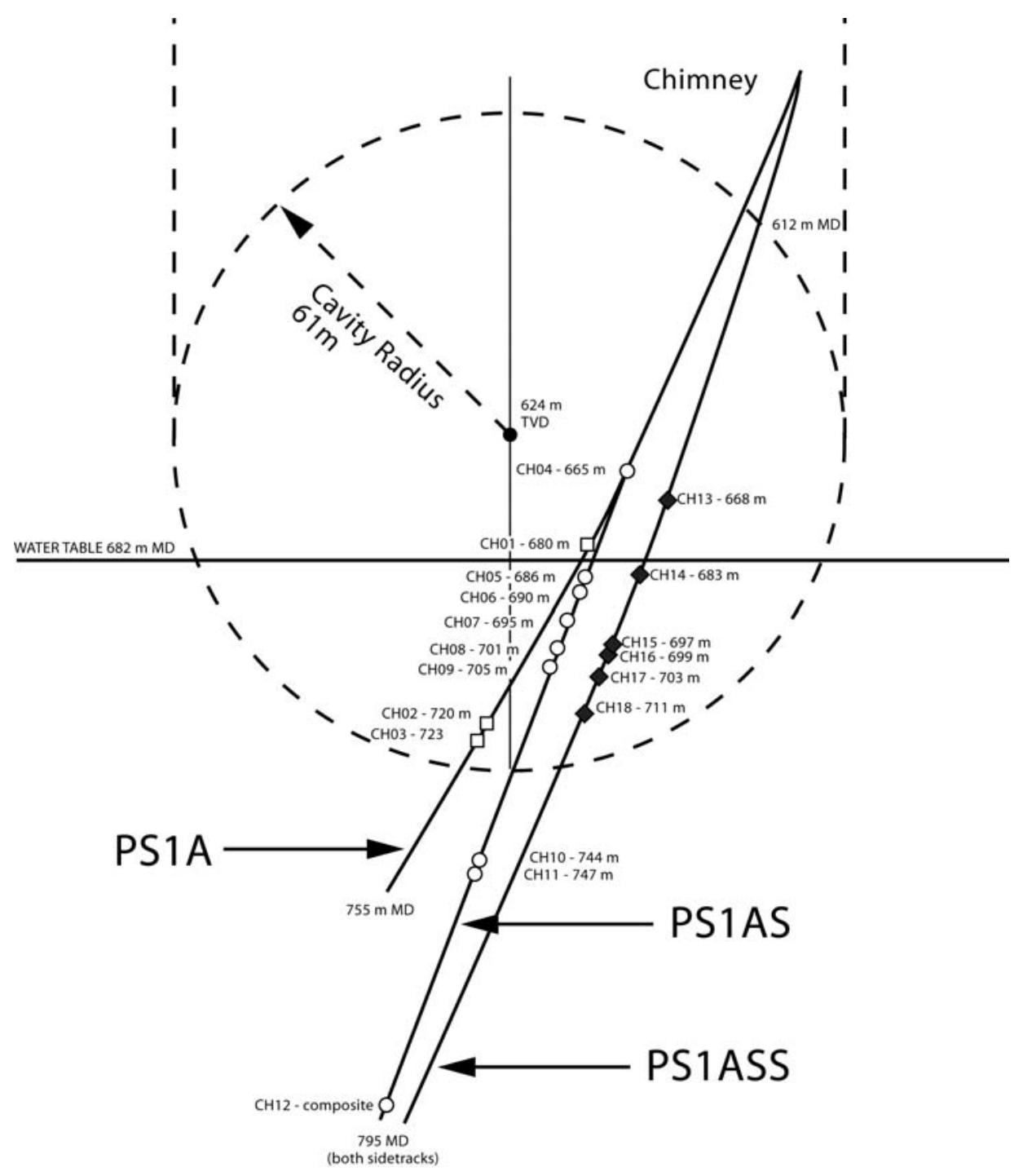

Figure 3.1 Cross section of the CHANCELLOR cavity, chimney, and post-shot boreholes showing the locations of sidewall core samples that were used in this investigation. Main hole (PS1A); first sidetrack (PS1AS) and second sidetrack (PS1ASS). MD: measured depth, TVD: total vertical depth. 
Table 3.2 Technetium and Iodine Data of Solid Samples from the CHANCELLOR Test Cavity

\begin{tabular}{|c|c|c|c|c|c|c|}
\hline $\begin{array}{l}\text { Sample } \\
\text { ID }\end{array}$ & sample description & $\begin{array}{c}{ }^{99} \mathrm{Tc} \\
\text { (ng/kg) }\end{array}$ & $\begin{array}{c}{ }^{99} \mathrm{Tc} \\
(\mathrm{Bq} / \mathrm{kg})\end{array}$ & $\begin{array}{c}\text { Total Iodine } \\
(\mu \mathrm{g} / \mathrm{kg})\end{array}$ & ${ }^{129} I /{ }^{127} I$ & $\begin{array}{c}{ }^{129} \mathrm{I} \\
(\mathrm{mBq} / \mathrm{kg})\end{array}$ \\
\hline Blank & & $<1$ & $<1$ & & $3.0 \mathrm{E}-13$ & \\
\hline $\mathrm{CH} 01 \mathrm{a}$ & gray volcanic rocks & 9 & 5 & 23.6 & 3.1E-07 & 0.049 \\
\hline $\mathrm{CH} 01 \mathrm{~b}$ & $\begin{array}{l}\text { gray volcanic rocks with some black } \\
\text { melt glass on surface }\end{array}$ & 1.9 & 12 & 26 & 3.7E-08 & 0.0064 \\
\hline $\mathrm{CH} 03 \mathrm{a}$ & $\begin{array}{l}\text { reddish-brown melt glass, non- } \\
\text { vesicular }\end{array}$ & $729 \pm 31$ & $458 \pm 20$ & 14.3 & 8.8E-04 & 83 \\
\hline $\mathrm{CH} 03 \mathrm{~b}$ & $\begin{array}{l}\text { pale-gray volcanic rock mix with small } \\
\text { portion of melt glass }\end{array}$ & $39 \pm 4$ & $24 \pm 3$ & & & \\
\hline $\mathrm{CH} 04 \mathrm{a}$ & vesicular brown melt glass & 669 & 420 & 40.3 & 1.7E-04 & 45 \\
\hline $\mathrm{CH} 04 \mathrm{~b}$ & $\begin{array}{l}\text { volcanic rock, some partially melted to } \\
\text { form light-colored vesicular glass }\end{array}$ & $61 \pm 3$ & $38 \pm 2$ & 12.6 & 1.1E-04 & 9.1 \\
\hline $\mathrm{CH} 06 \mathrm{a}$ & black melt glass & 597 & 375 & 5 & 1.3E-03 & 44 \\
\hline $\mathrm{CH} 06 \mathrm{~b}$ & $\begin{array}{l}\text { black melt glass mixed with volcanic } \\
\text { rock }\end{array}$ & 632 & 397 & 3.4 & $7.2 \mathrm{E}-04$ & 16 \\
\hline $\mathrm{CH} 09 \mathrm{a}$ & dark brown / black melt glass & 742 & 466 & 0.9 & $3.9 \mathrm{E}-03$ & 23 \\
\hline $\mathrm{CH} 09 \mathrm{~b}$ & dark brown / black melt glass & 695 & 436 & & & \\
\hline $\mathrm{CH} 12$ & black melt glass & $645 \pm 17$ & $405 \pm 11$ & 14.1 & & \\
\hline $\mathrm{CH} 14$ & $\begin{array}{l}\text { volcanic rock, some partially melted to } \\
\text { form light-colored non-vesicular glass }\end{array}$ & $49 \pm 3$ & $31 \pm 2$ & 16.7 & 4.4E-05 & 4.9 \\
\hline $\mathrm{CH} 16$ & dark brown/black melt glass & 747 & 469 & 3 & $2.4 \mathrm{E}-03$ & 49 \\
\hline
\end{tabular}


The ${ }^{99}$ Tc concentrations correlate with sample type. ${ }^{99} \mathrm{Tc}$ in the dark melt glass exhibits a fairly uniform distribution, with a narrow range of $600-750 \mathrm{ng} / \mathrm{kg}$. The non-melted volcanic rock contains $<100 \mathrm{ng} / \mathrm{kg}{ }^{99} \mathrm{Tc}$. The ${ }^{129} \mathrm{I}$ activities in the solid samples range from 0.006 to $83 \mathrm{mBq} / \mathrm{kg}$ with highest concentrations found in the dark melt glass samples. The two samples ( $\mathrm{CH} 01 \mathrm{a}$ and b) that have very low ${ }^{129} \mathrm{I}$ activities also have the lowest ${ }^{99} \mathrm{Tc}$ concentrations of all samples.

\subsubsection{Radionuclide Distribution in a Test Cavity}

Based on the announced yield of the CHANCELLOR test (143 kt of TNT equivalent) (DOE/NV, 2000), the depth of burial, and the bulk density of the overlying rock, we estimate the test cavity radius (Pawloski, 1999) and generated mass of melt glass (Olsen, 1993 ) to be $61 \mathrm{~m}$ and $\sim 1.43 \times 10^{8} \mathrm{~kg}$, respectively. Assuming a density of $2.5 \mathrm{~g} / \mathrm{cm}^{3}$ and porosity of $30 \%$ for the melt glass and test debris, we can estimate the volume of melt glass and rubble in the cavity. The volume of groundwater in the cavity can be calculated assuming the saturated zone porosity is fully saturated and unsaturated zone is only $50 \%$ saturated.

Table 3.3 Distribution of Radionuclides in the CHANCELLOR Test Cavity

\begin{tabular}{cccccccc}
\hline & $\begin{array}{c}\text { Total } \\
\text { Mass (g) }\end{array}$ & $\begin{array}{c}\text { Total } \\
\text { (MBq) }\end{array}$ & ${ }^{99}$ Tc\% & $\begin{array}{c}{ }^{99} \text { Tc\% } \\
\text { IAEA }\end{array}$ & $\begin{array}{c}\text { Total } \\
\text { (MBq) }\end{array}$ & ${ }^{129}{ }^{12} \%$ & $\begin{array}{c}{ }^{129} \mathbf{I} \% \\
\text { IAEA }\end{array}$ \\
\hline Melt Glass & $1.43 \mathrm{E} 11$ & $6.14 \mathrm{E} 4$ & $65 \%$ & 80 & 7.0 & $28 \%$ & 50 \\
Rubble & $1.52 \mathrm{E} 12$ & $3.35 \mathrm{E} 4$ & $35 \%$ & 20 & 5.3 & $24 \%$ & 40 \\
Groundwater & $1.80 \mathrm{E} 11$ & $3.07 \mathrm{E} 2$ & $0.3 \%$ & 0 & 13.0 & $48 \%$ & 10 \\
\hline
\end{tabular}

Using the measured ${ }^{99} \mathrm{Tc}$ and ${ }^{129} \mathrm{I}$ concentrations in the melt glass, rubble, and groundwater, the partitioning of ${ }^{99} \mathrm{Tc}$ and ${ }^{129} \mathrm{I}$ can be estimated (Table 3.3) and compared with IAEA estimates (IAEA, 1998). Approximately $65 \%$ of the ${ }^{99} \mathrm{Tc}$ is associated with the melt glass, and $35 \%$ is associated with the rubble. Our estimate agrees with IAEA values to within $\pm 15 \%$. Only a very small proportion of ${ }^{99} \mathrm{Tc}(0.3 \%)$ was dissolved in the cavity water, which is expected given its strong sorption under moderately reducing conditions (Lieser and Bauscher, 1987). Based on rubble and water concentrations, we estimate the ${ }^{99} \mathrm{Tc} \mathrm{K}_{\mathrm{d}}$ to be between 3 and $22 \mathrm{~mL} / \mathrm{g}$. Both $\mathrm{Eh}$ and iodine speciation measurements support the conclusion that the groundwater was reducing.

Using the same approach for ${ }^{129} \mathrm{I}$, we estimate $28 \%, 24 \%$ and $48 \%$ of ${ }^{129} \mathrm{I}$ is in the melt glass, rubble and groundwater phases, respectively. Compared with IAEA estimates (IAEA, 1998), our results show that a larger fraction of the ${ }^{129} \mathrm{I}$ is present in groundwater, and a lower fraction is contained in the glass and rubble. Given the high volatility of iodine and its mass 129 fission product precursors, together with the solubility of iodine in groundwater, the high fraction of ${ }^{129} \mathrm{I}$ in the cavity fluid would appear to be reasonable. Based on rubble and water concentrations, we estimate the ${ }^{129} \mathrm{I} \mathrm{K}_{\mathrm{d}}$ to be between 0 and $0.12 \mathrm{~mL} / \mathrm{g}$. However, ${ }^{129} \mathrm{I}$ was measured in only a few rubble samples, and our procedure lacked an iodine tracer to monitor recovery. Incomplete recovery of ${ }^{129} \mathrm{I}$ from solid samples would lead to an overestimation of ${ }^{129}$ I partitioning into the groundwater phase 
and an underestimation of $\mathrm{K}_{\mathrm{d}}$. Quartz-tube combustion experiments for measuring lowlevel total iodine in rock samples are underway and will be used to compare with TMAH microwave digestion results described here.

While the partitioning estimates developed during this study are approximate, it is notable that the values differ from those reported by the IAEA for French underground tests. To some extent, these differences may reflect variations in the underground test conditions. For example, the type of geologic media and the water or $\mathrm{CO}_{2}$ content of the rock can influence the amount of time that the cavity stands prior to collapse of the overlying rock. Cavity collapse results in the rapid cooling of the system, and disrupts communication between the vapor phase and melt. The timing of this 'quenching' event has a significant effect on the fission product distribution because the partitioning of these fission products is largely determined by the chemical behavior of fission chain precursor elements (Moody, 2003).

For example, most of the mass 99 and 129 fission chains are 'born' as short-lived precursors that decay to ${ }^{99} \mathrm{Tc}$ and ${ }^{129} \mathrm{I}$ on relatively short-time scales (half-lives of seconds to days). The chemical properties of these precursor elements will determine how the mass 99 and 129 species are distributed between the melt and vapor phases at the time of cavity collapse. Since each precursor species decays on a different time-scale, test cavities that are 'quenched' soon after the detonation will have a different fission product distribution than those that stand for a longer period of time. The future evaluation of radionuclide partitioning in underground test cavities that represent diverse cooling histories would provide additional constraints on the range in partitioning behavior.

\subsection{Conclusions}

${ }^{99} \mathrm{Tc}$ and ${ }^{129} \mathrm{I}$ are important contributors to risk assessment due to their long half-lives and high mobility as aqueous anionic species. ${ }^{99} \mathrm{Tc}$ and ${ }^{129} \mathrm{I}$ in groundwater samples from 13 wells and in test debris from the CHANCELLOR underground nuclear test cavity were analyzed using ICP-MS, LSC and AMS. In general, concentrations in the satellite wells are lower than in the test cavities for both ${ }^{99} \mathrm{Tc}$ and ${ }^{129} \mathrm{I}$. The ${ }^{129} \mathrm{I} /{ }^{127} \mathrm{I}$ ratio ranges from $10^{-3}$ to $10^{-6}$ in cavity water and $10^{-4}$ to $10^{-9}$ in satellite wells. The ${ }^{99} \mathrm{Tc}$ concentration ranges from 3 to $10^{-4} \mathrm{~Bq} / \mathrm{L}$ in cavity waters and from 0.3 to $10^{-4} \mathrm{~Bq} / \mathrm{L}$ in satellite wells. Downstream migration is apparent for both radionuclides. However, it is affected by both retardation and initial distribution. For example, ${ }^{99} \mathrm{Tc}$ and ${ }^{129} \mathrm{I} \mathrm{K}_{\mathrm{d}} \mathrm{s}$ calculated using rubble and water concentrations from CHANCELLOR are 3 to $22 \mathrm{~mL} / \mathrm{g}$ and 0 to $0.12 \mathrm{~mL} / \mathrm{g}$, respectively, and are suggestive of mildly reducing conditions. Though both radionuclides appear to be relatively mobile, ${ }^{99} \mathrm{Tc}$ is likely to be retarded as a result of its reduction from the $\mathrm{TcO}_{4}{ }^{-}$anion to the more sorbing and insoluble $\mathrm{TcO}_{2}$ species.

Using the analytical results of the water and test debris samples from CHANCELLOR, we have estimated the distribution of ${ }^{99} \mathrm{Tc}$ and ${ }^{129} \mathrm{I}$ between the melt glass, rubble and groundwater in the CHANCELLOR test cavity and compared them to reported values for underground tests in French Polynesia (IAEA, 1998). ${ }^{129}$ I distribution in the melt glass, rubble and groundwater of the Chancellor test cavity is $28 \%, 24 \%$ and $48 \%$, respectively; 
for ${ }^{99} \mathrm{Tc}$, it is $65 \%, 35 \%$ and $0.3 \%$, respectively. Our partitioning estimates differ from those of the IAEA (1998), implying that fission product distribution may vary from test to test. Factors that may influence this distribution include geologic conditions (e.g. lithology, water and $\mathrm{CO}_{2}$ content) and the cooling history of the test cavity.

\subsection{References}

ASTM (2005) Standard Guide for the Determination of Technetium-99 in Soil. ASTM International, West Conshohocken, PA, 2005.

Bergquist B. A., Marchetti A. A., Martinelli R. E., McAninch J. E., Nimz G. J., Proctor I. D, Southon J. R., Vogel J. S. (2000), Nucl. Instrum. Meth. B, 172:328.

Dixon P., Curtis D. B., Musgrave J., Roensch F., Roach J., Rokop D. (1997) Anal. Chem., 69 (1997) 1692.

DOE/NV (2000), United States Nuclear Tests, July 1945 through September 1992. U.S. Department of Energy, Nevada Operations Office, Las Vegas. DOE/NV-209 (Rev. $15)$

Eichrom Technologies (2002) Technetium-99 in Water. Analytical Procedures, Procedure Number TCW01, 2002.

IAEA (1998), The Radiological Situation at the Atolls of Mururoa and FangataufaInventory of Radionuclides underground at the Atolls, International Atomic Energy Agency Report IAEA-MFTR-3, Vienna, 1998.

Lieser K. H. and Bauscher CH. (1987) Radiochimica Acta, 42 (1987) 205.

Marchetti A. A., Gu F., Robl R., Straume T. (1997) Nucl.Instrum. Meth.B, 123:352.

Mas J. L., Tagami K. and Uchida S. (2004) Analytica Chimica Acta, 509 (2004) 83.

Moody K. J. (2003) Underground Chemistry and the Uncertainty of the Yields of Nuclear Devices, Lawrence Livermore National Laboratory Report, UCRL-TR-200852.

Moran J.E., Fehn U., Hanor J.S. (1995) Geochimica et Cosmochimica Acta, 59 50555069.

Muramatsu Y. and Wedepohl K. H. (1998), Chem. Geol., 147:201.

Olsen C. W. (1993) Site Selection and Containment Evaluation for LLNL Nuclear Events. Proceedings of the Seventh Symposium on Containment of Underground Nuclear Explosions, vol 1, p.85-119.

Pawloski G. A. (1999) Development of Phenomenological Models of Underground Nuclear Tests on Pahute Mesa, Nevada Test Site - BENHAM and TYBO, Lawrence Livermore National Laboratory Report, UCRL-ID-136003.

Uchida T., Isoyama H., Yamada K., Oguchi K., Nakagawa G., Sugie H., Iida C. (1992), Analytica Chimica Acta, 256: 277. 
Yamada H., Kiriyama T., Onagawa Y., Hisamori I., Miyazaki C., Yonebayashi K. K. (1999). Soil Sci. Plant Nutri., 45:563.

Zhou, C.Y., Wang M. K., Koh L. L., Weeb Y. C. (1996) Talanta, 43:1061. 


\title{
Chapter 4
}

\section{Characterization of Experimentally Altered Nuclear Melt Glass}

\author{
L. Shuller, P. Zhao, A. B. Kersting, and M. Zavarin \\ Chemical Biology and Nuclear Science Division \\ Lawrence Livermore National Laboratory
}

\subsection{Introduction}

The nuclear melt glass that forms as a result of an underground nuclear test retains the majority of the actinide residual inventory (Borg, 1975). A major concern in risk assessment at these sites is the transport of actinides in groundwater. Secondary mineral precipitates that form as a result of melt glass dissolution can sequester insoluble radionuclides (e.g. plutonium) and minimize their migration. Secondary mineral precipitates may also form colloids and promote transport of radionuclides in groundwater. Understanding glass dissolution behavior and identifying the associated secondary mineral phases is essential to the accurate prediction of radionuclide transport.

The two major objectives of the experiments described in this chapter were: (1) understand the glass dissolution behavior of nuclear melt glass from the NTS and (2) characterize the secondary mineral phases that precipitate as a result of nuclear melt glass dissolution at elevated temperatures $\left(120^{\circ} \mathrm{C}\right.$ and $\left.200^{\circ} \mathrm{C}\right)$. A previous study was conducted using a wider temperature range $\left(40^{\circ} \mathrm{C}\right.$ to $\left.200^{\circ} \mathrm{C}\right)$ over a 3 -month reaction period (Zavarin et al., 2004). This reaction time yielded little alteration at all temperatures except $200^{\circ} \mathrm{C}$. The current experiments extended the reaction time at the two highest temperatures examined previously.

\subsection{Methods}

The experiments described herein examined the supernatant composition and the secondary mineral characteristics resulting from high temperature alteration of nuclear melt glass. Ion Chromatography (IC) and Inductively Coupled Plasma - Mass Spectroscopy (ICP-MS) were used to determine the chemical composition of the supernatant. The Eh and $\mathrm{pH}$ were also measured. Scanning Electron Microscopy (SEM) with the Energy Dispersive X-ray Spectroscopy (EDS) provided a means of qualitative chemical and morphological characterization of secondary mineral precipitates. X-ray Diffraction (XRD) was used to help identify the secondary mineral phases. Alpharadiography, along with liquid scintillation counting, was used to determine the concentration of $\alpha$-emitters in the solid and supernatant, respectively. 


\subsection{Procedure}

\subsubsection{Experimental protocol}

In an effort to identify the minerals that form from the high-temperature alteration of nuclear melt glass in groundwater, three samples of melt glass were individually reacted with a simple synthetic groundwater $\left(3 \times 10^{-3} \mathrm{~mol} / \mathrm{L} \mathrm{Na}, 2 \times 10^{-3} \mathrm{~mol} / \mathrm{L} \mathrm{HCO}_{3} / \mathrm{CO}_{3}{ }^{2-}\right)$ for 12 months at 120 and $200^{\circ} \mathrm{C}$. The synthetic groundwater is a simplified analog of groundwater present in the Pahute Mesa region of the NTS (Pawloski et al., 2001). Three samples were used in the dissolution experiments (Table 4.1). JMK\#1 and JMK\#3 nuclear melt glasses were selected from an undisclosed nuclear test location at the NTS. The chemical composition of the glasses was a high-silica rhyolite. Samples were crushed and sieved and the 25-53 microns particle size was used in all experiments. Glass samples were washed in dilute HF solution to remove any natural alteration products. Experiments were run in Parr ${ }^{\circledR}$ bombs placed in temperature controlled ovens. Details regarding the sample preparation and experimental procedure can be found in Zavarin et al. (2004).

Table 4.1 Glass dissolution experiment parameters

\begin{tabular}{ccc}
\hline Sample & Amount gram & Temp $^{\mathbf{0}} \mathbf{C}$ \\
\hline JMK\#3@200 $20{ }^{\circ} \mathrm{C}$ & 1 & 200 \\
JMK\#3@120 $\mathrm{C}$ & 1 & 120 \\
$\mathrm{JMK} \# 1 @ 120^{\circ} \mathrm{C}$ & 1 & 120 \\
\hline
\end{tabular}

\subsubsection{Sample Characterization}

After 12 months, the three samples were removed from the ovens and allowed to cool to room temperature. Samples were removed from their respective vessels and centrifuged in $30 \mathrm{~mL}$ vials. The supernatant was decanted into second vial and analyzed by IC, ICP-MS, and LSC. The major elements measured included sodium, potassium, magnesium, and calcium. The elements measured by ICP-MS included aluminum, silicon, titanium, iron, ${ }^{239} \mathrm{Pu},{ }^{238} \mathrm{U},{ }^{237} \mathrm{~Np}$, and ${ }^{241} \mathrm{Am}$. Eh and $\mathrm{pH}$ were also measured.

After 12 months, the solid residue in all three samples had visibly altered from the original glass (Figure 4.1). Milli-Q water was added to the vial to separate the primary glass from the secondary minerals. After shaking the vial, the larger glass particles were allowed to settle while the suspended solution (with secondary phases) was pipetted out. This was repeated until the majority of the high surface area (secondary mineral) particles were separated. The $200^{\circ} \mathrm{C}$ sample had 2 visually distinct secondary mineral precipitates. Sub-sample JMK\#3@200Ca had a tan hue, while sample JMK\#3@200Cb had a cream hue. 


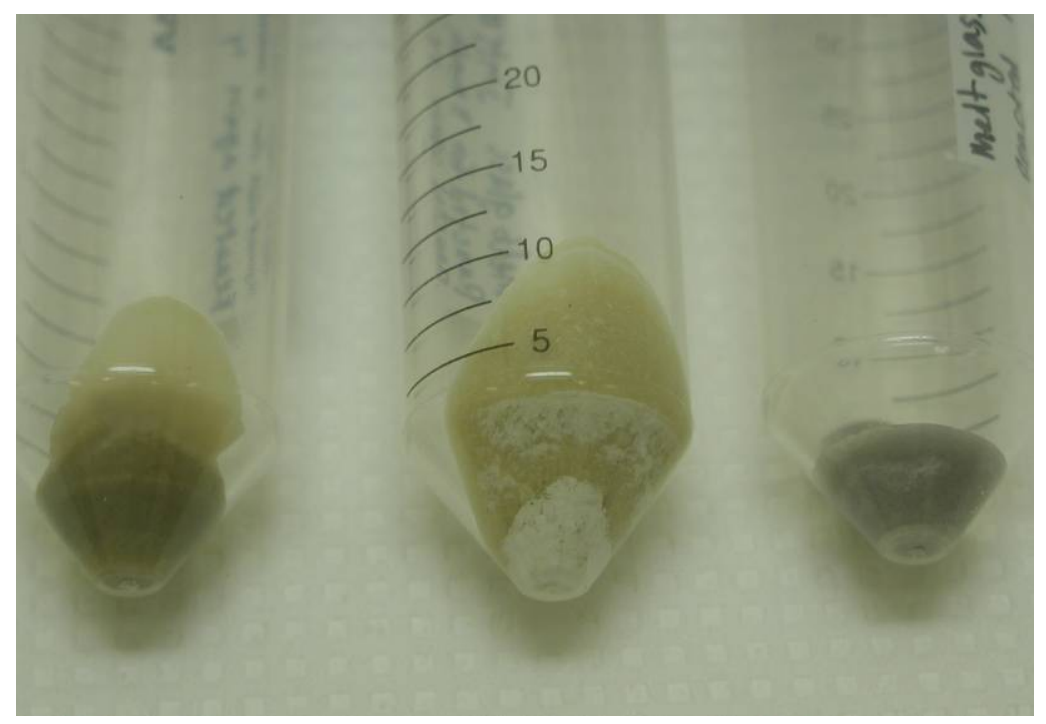

Figure 4.1 Centrifuge tubes containing primary and secondary minerals.

The secondary minerals were characterized by SEM, EDS, XRD, and $\alpha$-radiography. The first SEM samples were prepared on small gold coated carbon dots. Later SEM samples were prepared on graphite discs, two of which were gold coated. SEM provides information on secondary mineral morphology. The EDS provides chemical information for images observed in SEM. However, the EDS data provide only gross sample characterization because the secondary mineral particle sizes (microns to $10 \mathrm{~s}$ of nanometers) examined here were smaller than the EDS spatial resolution.

XRD was used to determine the secondary mineral phases present. In an earlier study (Zavarin et al., 2004), the secondary mineral phases identified by XRD did not differ significantly from the original phases present in the nuclear melt glass. Therefore, the secondary mineral products of glass dissolution could not be identified. In the present experiment, HF was used to remove secondary minerals from the starting material. Furthermore, at the end of the reaction period, secondary mineral precipitates were separated from the bulk melt glass. This allowed for much better secondary mineral identification. XRD of the two $120^{\circ} \mathrm{C}$ samples was accomplished by drying the wet samples on quartz plates. The quartz plates have the benefit of minimal XRD background. The $200^{\circ} \mathrm{C}$ samples were dried on 1" diameter carbon dots to make them less dispersive. A carbon dot standard was run and the background had no significant XRD features.

The technique of $\alpha$-radiography was used to detect the presence and location of $\alpha$ emitters in the solid samples. The $\alpha$-radiography samples were prepared using small SEM plugs and carbon dots. A drop of the separated secondary mineral was spread across the carbon dot and dried. The $\alpha$-radiography plastic film (TASTRAK CR-39), a polycarbonate plastic alpha-track detector, was placed on top of the dried samples and allowed to interact with the sample for 3.5 days or 15 hours. After exposing the film to the sample for the desired time, the film was etched by placing it into a bath of $6.25 \mathrm{~N}$ $\mathrm{NaOH}$ for 6 hours at $75^{\circ} \mathrm{C}$. The $\alpha$ tracks were observed by optical microscope. 


\subsection{Results and Discussion}

\subsubsection{Supernatant Composition}

All three experiments resulted in an alkaline supernatant (when cooled to $25^{\circ} \mathrm{C}$ ). However, the $120^{\circ} \mathrm{C}$ samples had significantly higher $\mathrm{pHs}$ that the $200^{\circ} \mathrm{C}$ sample (Table 4.2). Interestingly, Pawloski et al. (2001) reported that precipitation of clay minerals is favored at lower temperatures and leads to fairly alkaline $\mathrm{pHs}$ while precipitation of zeolites and framework silicates is favored at higher temperatures and leads to more acid solution conditions. The supernatant solution $\mathrm{pHs}$ measured here are consistent with that analysis.

The measured Ehs of all the samples were relatively similar. However, the Eh was measured in an open centrifuge tube over an extended period of time (about 10-30 minutes) and was observed to change with time (Eh measurements listed in Table 4.2 represent the final reading). Thus, the Eh reported here may not represent Eh conditions in the reaction vessels during heating. Nevertheless, results suggest that glass dissolution will result in at least mildly reducing conditions (Figure 4.2). The results compare well with those of Zavarin et al. (2004).

Table 4.2 pH and Eh results for nuclear melt glass supernatant

\begin{tabular}{lll}
\hline Sample & pH & Eh (mV) \\
\hline JMK\#3@200 & 8.48 & 190.8 \\
JMK\#3@120 C C & 9.85 & 168.0 \\
JMK\#1@120 $\mathrm{C}$ & 9.86 & 176.0 \\
\hline
\end{tabular}

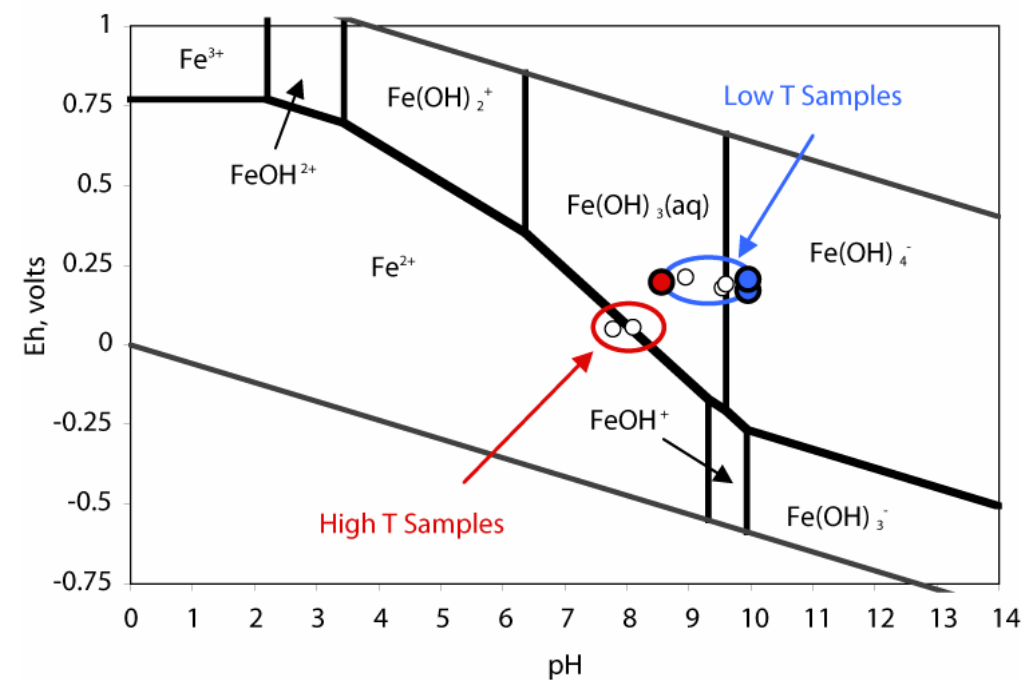

Figure 4.2 Eh-pH values for the $120^{\circ} \mathrm{C}$ (blue) and $200^{\circ} \mathrm{C}$ (red) samples superimposed on the Eh-pH diagram and data from Zavarin et al. (2004). 
The supernatant composition (Table 4.3) reflects the glass dissolution and secondary mineral precipitation behavior at elevated temperatures over time. The original synthetic groundwater had a composition of $3 \times 10^{-3} \mathrm{~mol} / \mathrm{L} \mathrm{Na}$ and $2 \times 10^{-3} \mathrm{~mol} / \mathrm{L} \mathrm{HCO}_{3}{ }^{-} / \mathrm{CO}_{3}{ }^{2-}$. It should be noted that some changes in solution composition may have occurred during sample cooling. For example, the relatively low Si concentration in the $200^{\circ} \mathrm{C}$ sample (relative to $120^{\circ} \mathrm{C}$ ) may have resulted from silica precipitation during sample cooling. Nevertheless, the following can be said:

- $\mathrm{NO}_{3} / \mathrm{NO}_{2}$ concentrations reflect mildly reducing conditions consistent with Eh measurements

- High measured Fe and Al concentrations and their known low solubilities suggest that colloidal material was present in the supernatant.

Table 4.3 Nuclear melt glass supernatant composition

\begin{tabular}{lccc}
\hline Element & $\begin{array}{c}\text { JMK\#1 } \\
120^{\circ} \mathrm{C}\end{array}$ & $\begin{array}{c}\text { JMK\#3 } \\
120^{\circ} \mathrm{C}\end{array}$ & $\begin{array}{c}\text { JMK\#3 } \\
200^{\circ} \mathrm{C}\end{array}$ \\
\hline $\mathrm{Na}^{\mathrm{a}}$ & 156 & 203 & 110 \\
$\mathrm{Mg}^{\mathrm{a}}$ & 0.04 & 2.82 & 1.15 \\
$\mathrm{Al}^{\mathrm{a}}$ & 9.4 & 26 & 9.8 \\
$\mathrm{~K}^{\mathrm{a}}$ & 15.8 & 14.2 & 11.5 \\
$\mathrm{Ca}^{\mathrm{a}}$ & 1.03 & 4.24 & 0.68 \\
$\mathrm{Ti}^{\mathrm{a}}$ & 4.43 & 4.7 & 4.94 \\
$\mathrm{Fe}^{\mathrm{a}}$ & 1.93 & 12.8 & 16 \\
$\mathrm{Si}^{\mathrm{a}}$ & 207 & 219 & 188 \\
$238 \mathrm{U}^{\mathrm{a}}(\mathrm{ppb})$ & 5.18 & 5.71 & 22.4 \\
$\mathrm{~F}^{-\mathrm{b}}$ & 1.3 & 1.4 & 0.9 \\
$\mathrm{Cl}^{-\mathrm{b}}$ & 3.9 & 20 & 7.5 \\
$\mathrm{NO}_{2}{ }^{-b}$ & 0.23 & 0.68 & 0.66 \\
$\mathrm{NO}_{3}{ }^{-b}$ & 12.5 & 2.3 & 7.2 \\
$\mathrm{SO}_{4}{ }^{2-\mathrm{b}}$ & 0.78 & 2.1 & 3.8 \\
$\mathrm{PO}_{4}{ }^{3-\mathrm{b}}$ & 0.53 & 5.9 & 0.52 \\
\hline${ }^{\mathrm{a}} \mathrm{ICP}^{\mathrm{IC}}$ & & & \\
$\mathrm{IC}$ & & &
\end{tabular}

\subsubsection{SEM and EDS}

The SEM analysis provides insight into the morphology of the secondary minerals. Two samples at each temperature were prepared. The four samples are identified as JMK\#1@120ㄷ, JMK\#3@120C, JMK\#3@200 Ca, and JMK\#3@200Cb. Using the SEM images, the secondary mineral phases were identified by visually comparing the 
SEM images to ones found in the literature (Mumpton 1977). The phases were later verified using XRD.

The JMK\#1@120 ${ }^{\circ} \mathrm{C}$ sample had the least alteration material after the one-year reaction time. On the glass edges two distinct secondary mineral morphologies were observed (Figure 4.3). The first has sharp defined spikes about 1 to 3 microns in length, which are presumed to be mordenite. The second distinct morphology consists of thin wavy ridges, which could be classified as clay as it closely resembles smectite. Figure 4.2C depicts both morphologies on a particle of primary glass.
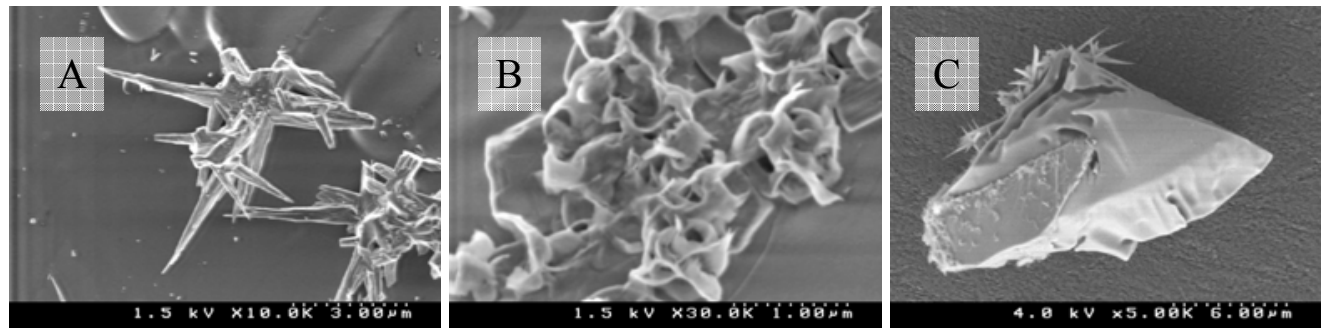

Figure 4.3 SEM Image of (A) mordenite morphology, (B) clay-like morphology, and $(\mathrm{C})$ primary glass in $\mathrm{JMK} \# 1 @ 120^{\circ} \mathrm{C}$.

The JMK\#3@120 ${ }^{\circ} \mathrm{C}$ contains more secondary minerals than the JMK\#1 sample (Figure 4.4). Many particles with clay-like morphology, identified by the layered features, were observed in this sample. Two other mineral morphologies were observed. The first consists of clusters of spikes or small fibers, which are presumed to be mordenite. While the second is the frayed edges of laminate mineral or glass sheets. It is not clear whether this morphology is representative of a specific mineral or simply the glass dissolution weathering pattern; Zavarin et al. (2004) presumed that it was a glass weathering pattern.
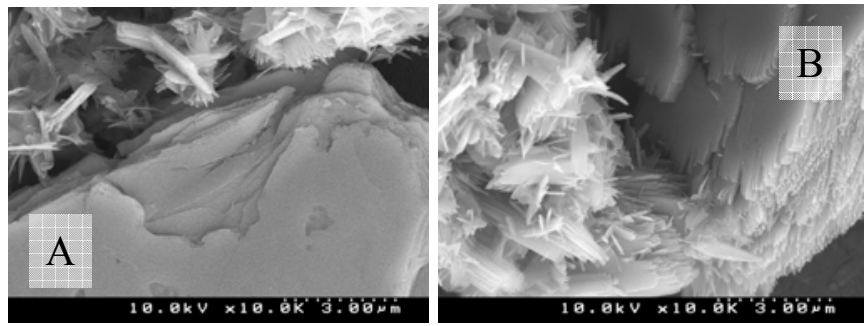

Figure 4.4 SEM image of (A) clay structure and (B) 2 other morphologies observed in JMK\#3@120 ${ }^{\circ} \mathrm{C}$.

The JMK\#3@200C samples contain the most secondary minerals. Sample A, the tan colored sample, has three prominent morphologies (Figure 4.5). First are the 50-200nm radius fibers. The fibers, presumably mordenite, are observed throughout the sample, and the other morphologies are intertwined with the fibrous phase. The second morphology consists of blocky crystals in groups and within the fibrous regions. The well-defined blocky crystals are fairly uniform in size at about 1 by 2 microns and are presumed to be clinoptilolite. The third morphology consists of small clusters of nanospheres. These 
grape-like clusters are located in cracks and crevices of the blocky crystals and the fibers. The mineral phase is undetermined.
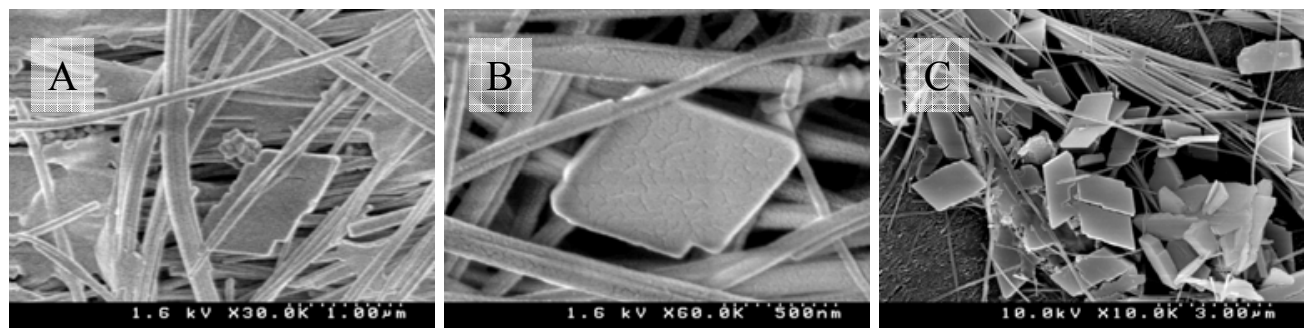

Figure 4.5 SEM image of (A) fibrous mordenite, (B) blocky clinoptilolite, and (C) grape-like clusters on clinoptilolite in sample JMK\#3@200Ca.

Sample B of the JMK\#3@ $200^{\circ} \mathrm{C}$ had the same morphologies as Sample A, but the dominant feature was blocky crystals, indicative of clinoptilolite, as opposed to the fibrous particles, indicative of mordenite (Figure 4.6). A particle of primary glass was found in this sample and the concoidal surface had an interesting microstructure. Additional SEM figures are located in the Appendix.
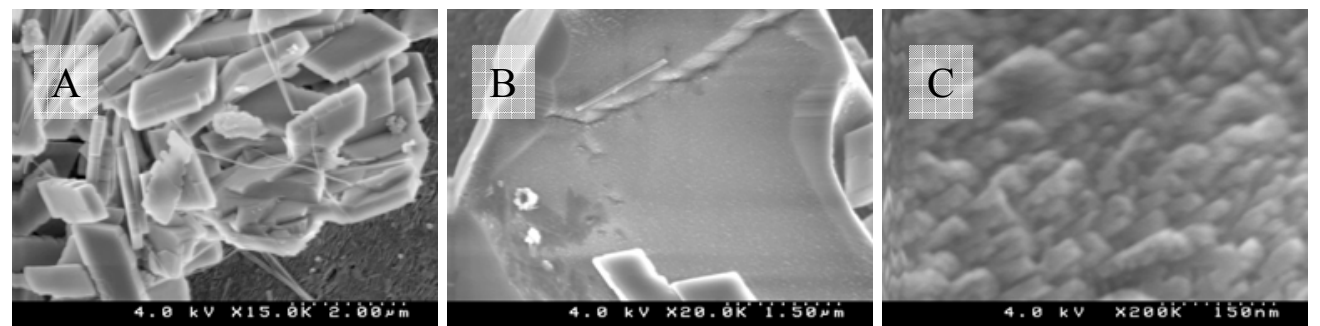

Figure 4.6 SEM image of (A) dominant blocky clinoptilolite, (B) concoidal primary glass and (C) weathering patterns from JMK\#3@200 $\mathrm{Cb}$.

The particle sizes shown in the previous section are generally smaller than the spatial resolution of the EDS elemental analysis capability. Thus, it is difficult, if not impossible, to resolve the chemical composition of the individual particles with specific growth morphologies. As a result, all 4 samples appear to have a relatively uniform chemical composition. We believe this to be an artifact of the EDS spatial resolution. Figure 4.7 is an example of a sample EDS spectra. The elements detected in all samples were $\mathrm{O}, \mathrm{Na}$, $\mathrm{Al}, \mathrm{Si}$, and $\mathrm{K}$. The clay-like particles and an amorphous particles in JMK\#3@120 $\mathrm{C}$ also contain some Fe, Mg, and Ti (Figure 4.8). To accurately characterize the mineral phases associated with the particular morphologies observed under SEM requires higher spatial resolution, such as that found with transmission electron microscopy (TEM). 


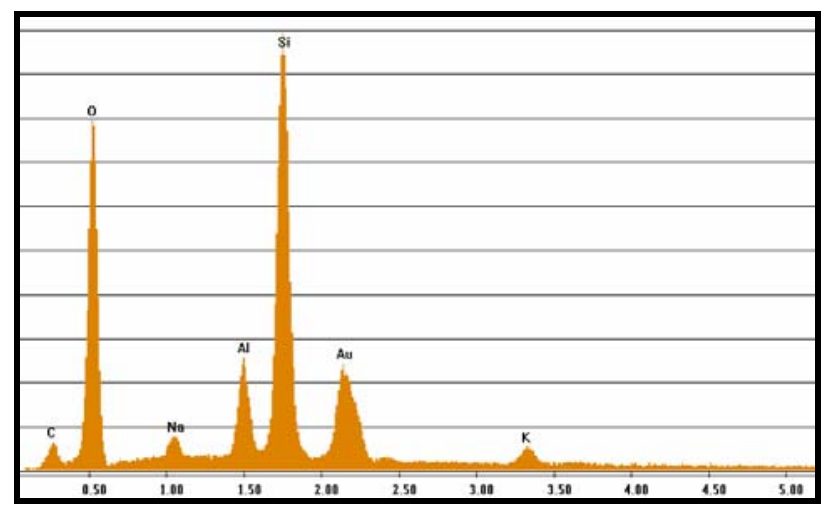

Figure 4.7 EDS Spectra of blocky crystal as seen in Figure 4.4, 10kV.

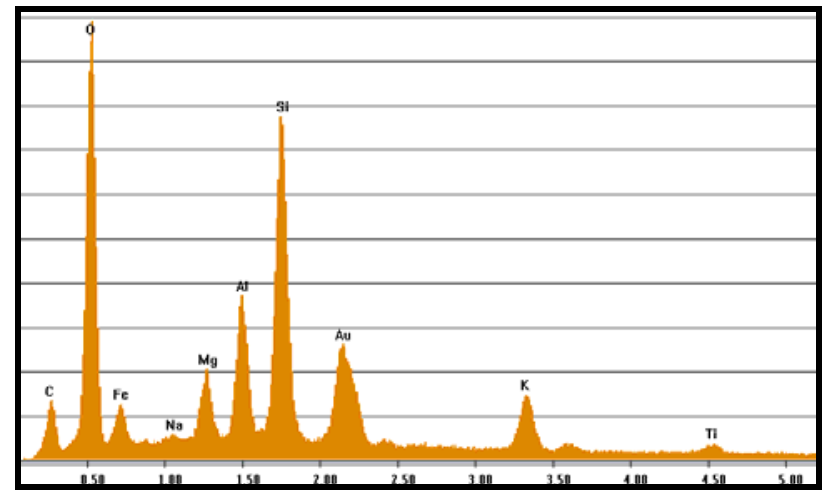

Figure 4.8 EDS spectra of a clay-like particle, $10 \mathrm{kV}$.

\subsubsection{XRD}

The XRD spectra of the four samples isolated from the reaction vessels are plotted in Figure 4.9. The JMK\#1@ $120^{\circ} \mathrm{C}$ spectrum contains no identifyable peaks with the exception of the single broad peak assigned to amorphous glass; it is estimated that alteration of the sample amounts to less than $10 \%$ of the sample. The other three spectra have peaks at nearly identical $2 \theta$ values, indicating similar alteration phases. The peak intensity is, to first order, correlated with the abundance of the phase associated with that peak. Thus, the more altered sample will tend to have higher peak intensities. However, changes in peak intensity will also be affected by the amount of material and its spatial distribution on the sample holder (e.g. a tightly packed sample will have higher peak intensities than a more loosely packed sample).

Using the JADE program, the secondary mineral phases were identified. The secondary mineral phases consisted of clinoptilolite, mordenite, albite, and illite-montmorillonite. The presence of additional zeolite and clay phases (i.e. heulandite, hydroxyapophyllite, and chlorite-vermiculite-montmorillonite) could not be ruled out. 

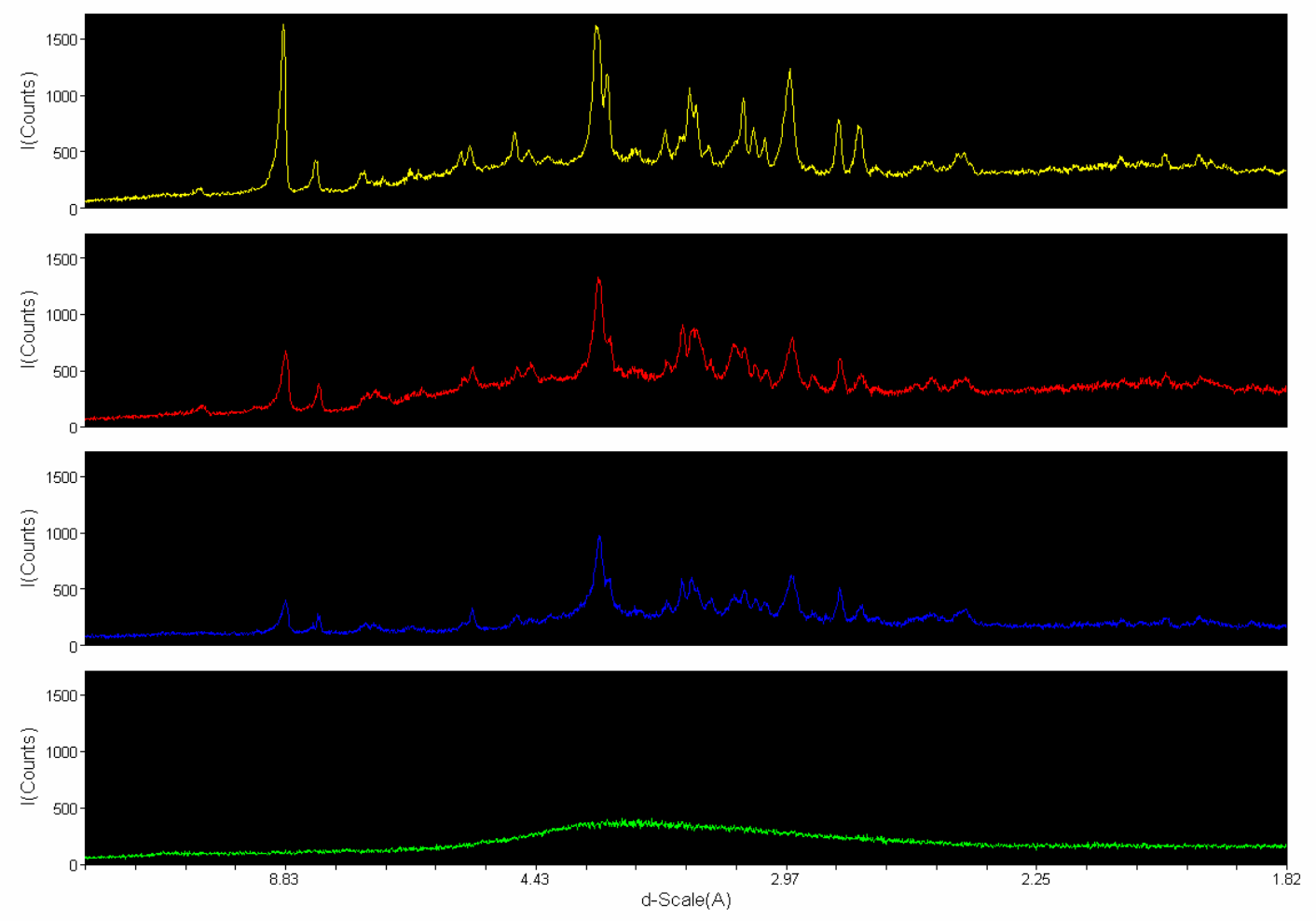

Figure 4.9 XRD spectra of reacted melt glass spectra; JMK\#3@200 Cb,
JMK\#3@200 Ca, JMK\#3@120 ${ }^{\circ} \mathrm{C}$, JMK\#1@120 $\mathrm{C}$ (top to bottom).

\subsubsection{Liquid Scintillation Counting}

Liquid scintillation counting of the bulk supernatant provides information regarding the overall activity of the supernatant. The counts in the $0-18.6 \mathrm{keV}$ range are primarily associated with tritium, while counts in the $50-500 \mathrm{keV}$ range are primarily associated with $\alpha$-emitters and high energy $\beta$-emitters. Identification of the isotope associated with the observed activity requires additional sample purification. However, the sharp peak seen in the $200^{\circ} \mathrm{C}$ sample spectrum (Figure 4.10) is believed to be an $\alpha$-emitting radionuclide. The counts per minute $(\mathrm{CPM})$ for each solution are listed in Table 4.4. The results were normalized to the amount of solution in contact with the nuclear melt glass solid during the experiment.

Importantly, the supernatant was separated from the solid by centrifugation. However, centrifugation only removed $>250 \mathrm{~nm}$ particles. Thus, the supernatant includes both the aqueous and the majority of colloidal-bound activity. As expected, the $200^{\circ} \mathrm{C}$ sample had more $\alpha$-emitting material in the supernatant than the samples reacted at the lower temperature. This alpha activity represents a mobile fraction comprised of both dissolved and colloidal material. These results indicate that glass dissolution provides a significant source of mobile alpha activity, particularly at high temperatures. 


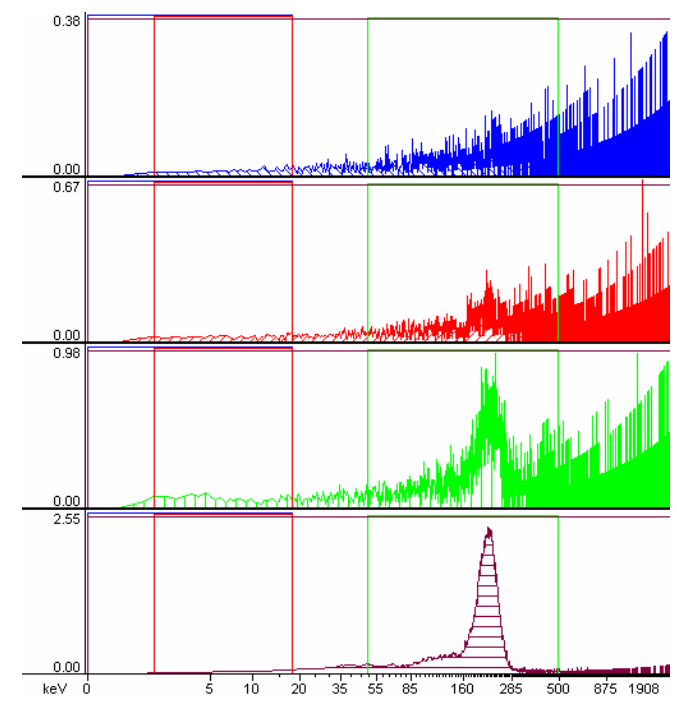

Figure 4.10 Liquid scintillation counting spectra for an activity-free standard, JMK\#1@120 ${ }^{\circ} \mathrm{C}$ supernatant, JMK\#3@120 ${ }^{\circ} \mathrm{C}$ supernatant, and JMK\#3@200 ${ }^{\circ} \mathrm{C}$ supernatant (top to bottom).

Table 4.4 Normalized liquid scintillation counting results

\begin{tabular}{ll} 
Sample & CPM (50-500 keV region) \\
\hline JMK\#1@120 $120^{\circ} \mathrm{C}$ & 5.5 \\
JMK\#3@120 $\mathrm{C}$ & 12 \\
JMK\#3@200 $200^{\circ} \mathrm{C}$ & 316 \\
\hline
\end{tabular}

\subsubsection{Alpha-radiography}

Two exposure times ( 3.5 days and 15 hours) yielded large numbers of $\alpha$-tracks in all radiography films of all samples (Figure 4.11). The only spatial information gained from these data was the surprisingly homogeneous $\alpha$-activity throughout the samples. However, the density of $\alpha$-tracks in the $200^{\circ} \mathrm{C}$ sample is significantly greater than in the $120^{\circ} \mathrm{C}$ samples. The $200^{\circ} \mathrm{C}$ sample particle surfaces are primarily composed of alteration mineral coatings while $120^{\circ} \mathrm{C}$ sample surfaces are largely unaltered. The results suggest that $\alpha$-emitting radionuclides, such as $\mathrm{U}$ and $\mathrm{Pu}$, are concentrated in the secondary mineral alteration compared to the glass. 
3.5 Day Exposure
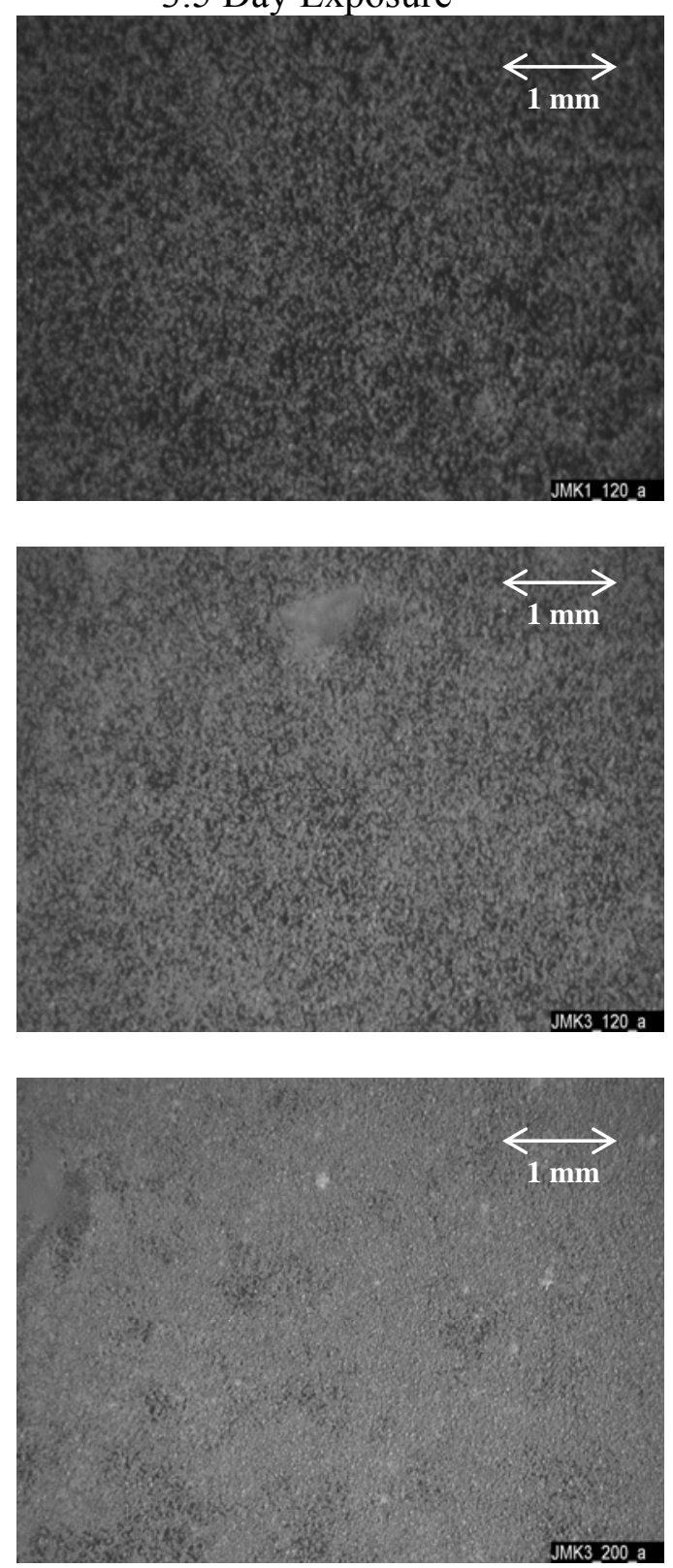

Figure 4.11 Alpha-radiography of altered nuclear melt glass samples.
15 Hour Exposure
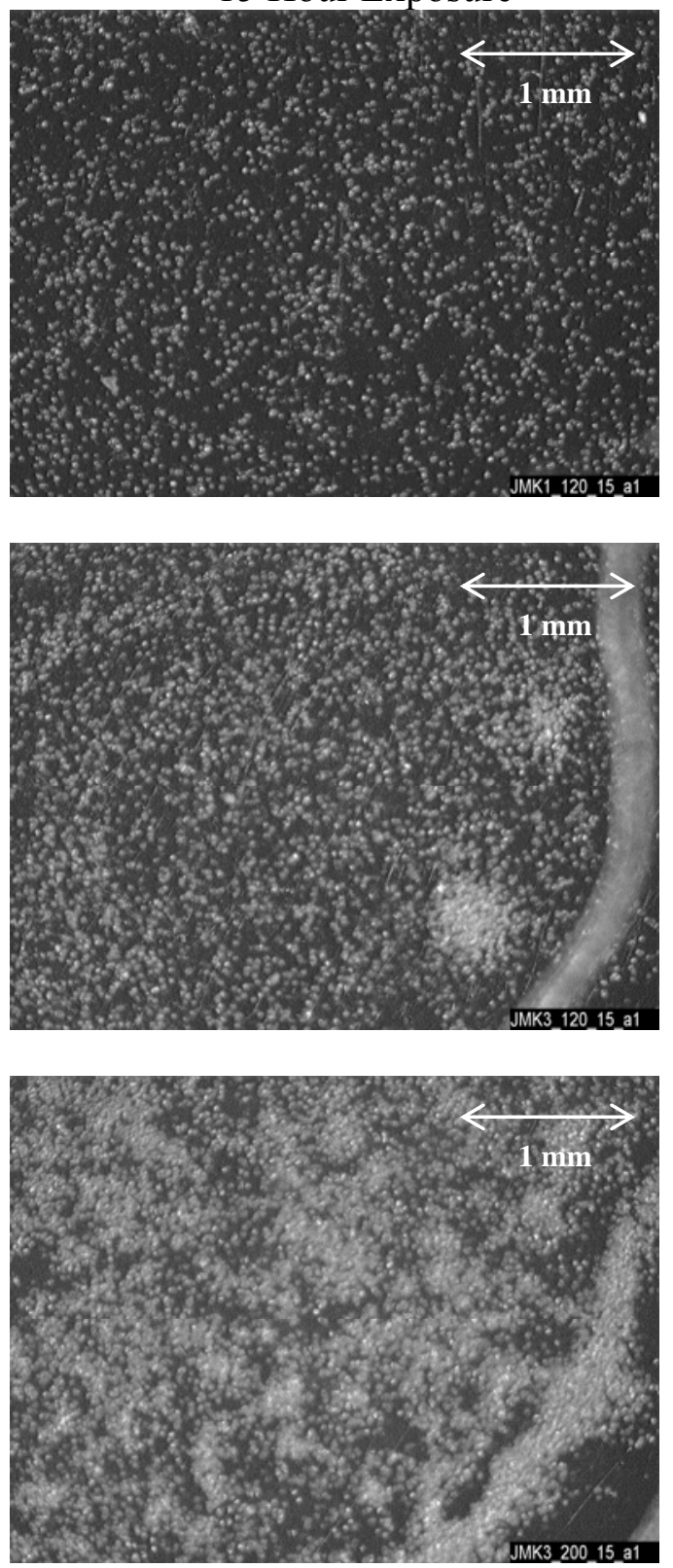


\subsection{Summary}

The secondary mineral phases from a 1-year nuclear melt glass dissolution experiment were characterized using $\mathrm{Eh} / \mathrm{pH}$, IC, ICP-MS, and LSC of the supernatant and SEM, EDS, XRD, and $\alpha$-radiography of the solids. Supernatant characterization indicates that glass dissolution produces mildly reducing conditions and that the aqueous +colloidal fractions include a substantial quantity of alpha activity. SEM and XRD data clearly indicate much greater glass alteration at $200^{\circ} \mathrm{C}$ compared to $120^{\circ} \mathrm{C}$. The secondary mineral phases identified were: clinoptilolite, mordenite, illite/montmorillolite, and albite. The mineralogy of the glass alteration products is generally same at both $120^{\circ} \mathrm{C}$ and $200^{\circ} \mathrm{C}$. However, the proportion of the mineral alteration products varies. Importantly, these phases are typical of fracture lining minerals identified in Pahute Mesa tuffs.

\subsection{References}

Borg I.Y. (1975). Radioactivity trapped in melt produced by a nuclear explosion, Nuclear Technology. 26:88-100.

Mumpton F.A., (1977). Natural Zeolites. Mineralogical Society of America Short Course Notes: Mineralogy and Geology of Natural Zeolites. Vol. 4 p. 14

Pawloski G.A., Tompson A.F.B., and Carle S.F. (2001) Evaluation of the Hydrologic source Term from Underground Nuclear Tests on Pahute Mesa at the Nevada Test Site: The Cheshire Test, Lawrence Livermore National Laboratory, UCRL-ID-147023, Livermore California.

Zavarin M., Roberts S.K., Viani B.E., Pawloski G.A., Rose T.P., Nuclear Melt Glass Dissolution and Secondary Mineral Precipitation at 40 to $200^{\circ} \mathrm{C}$ (2004). Lawrence Livermore National Laboratory. UCRL-TR-204870. 


\subsection{Appendix}

JMK\#1@120C

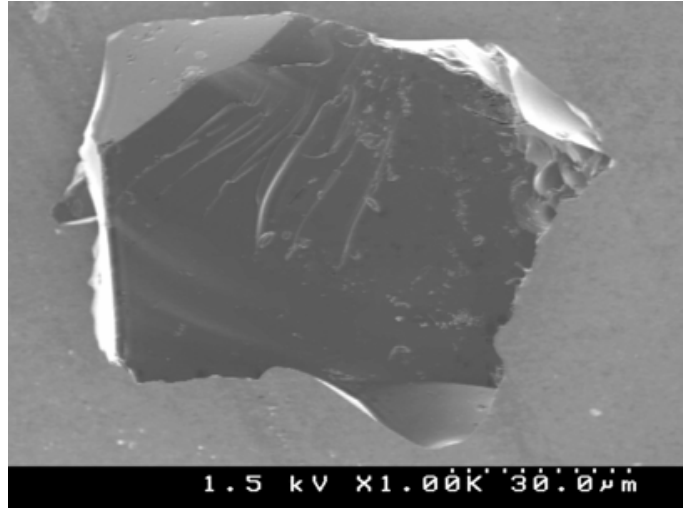

JMK\#1@120C

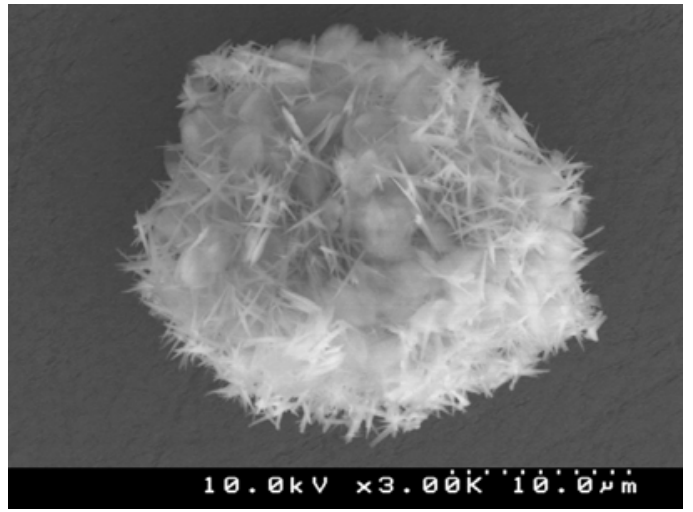

JMK\#3@120C

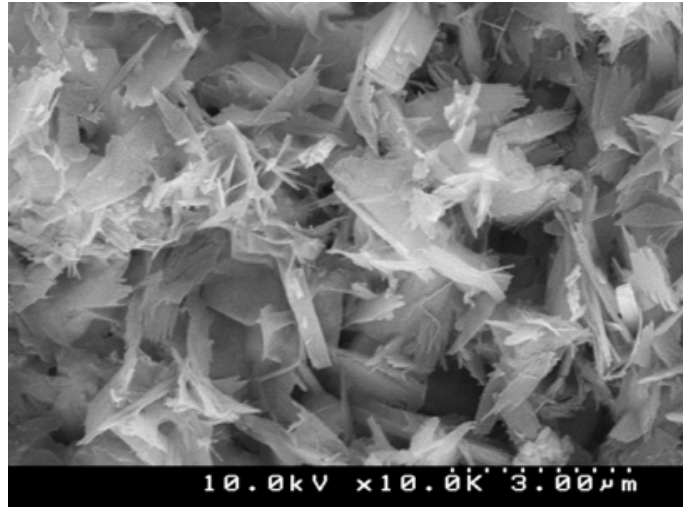

JMK\#1@120C

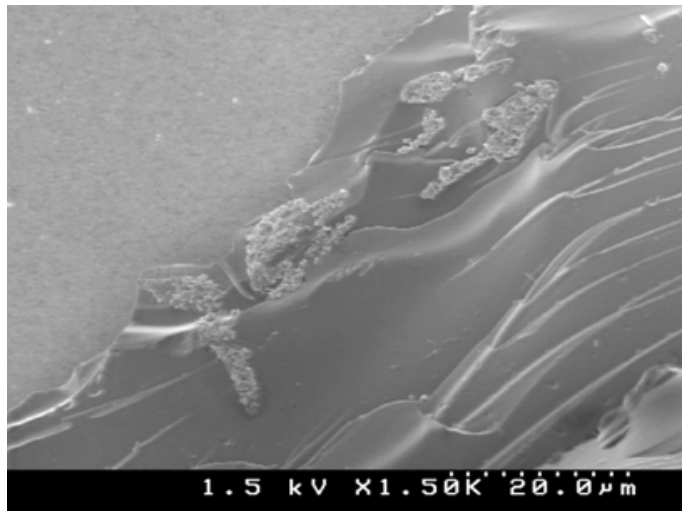

JMK\#3@120C

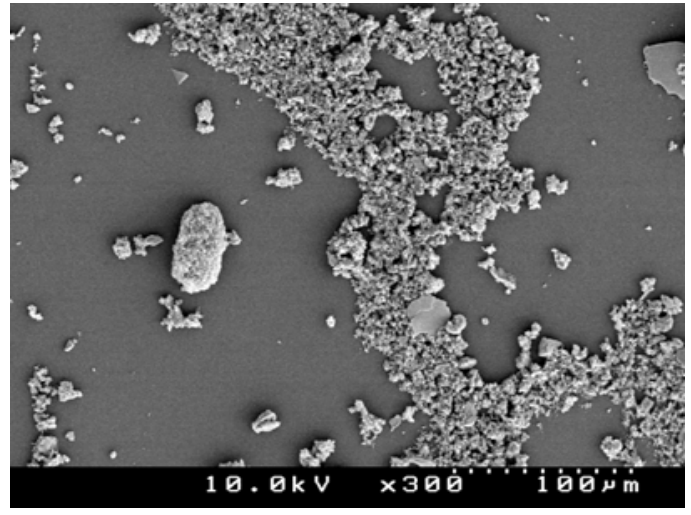

JMK\#3@120C

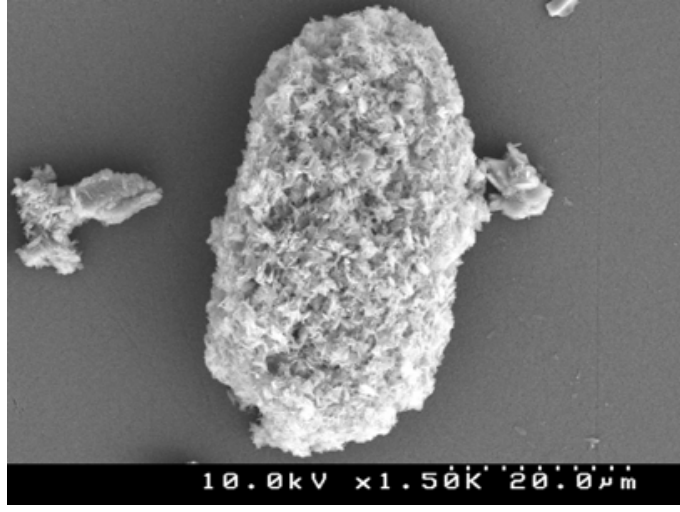


JMK\#3@120C

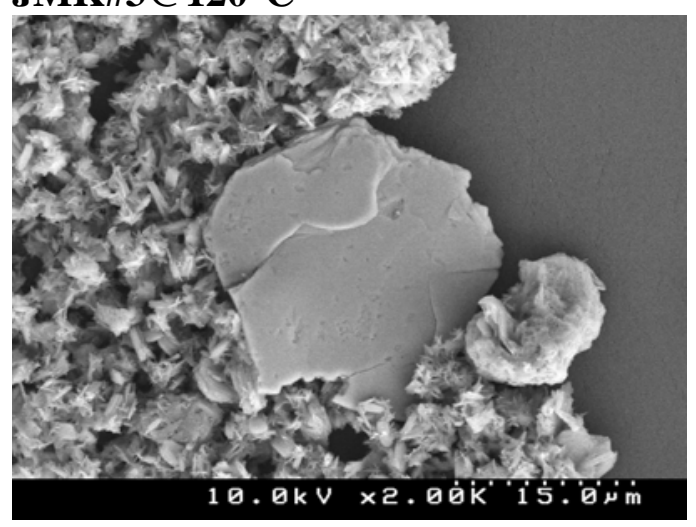

JMK\#3@120C

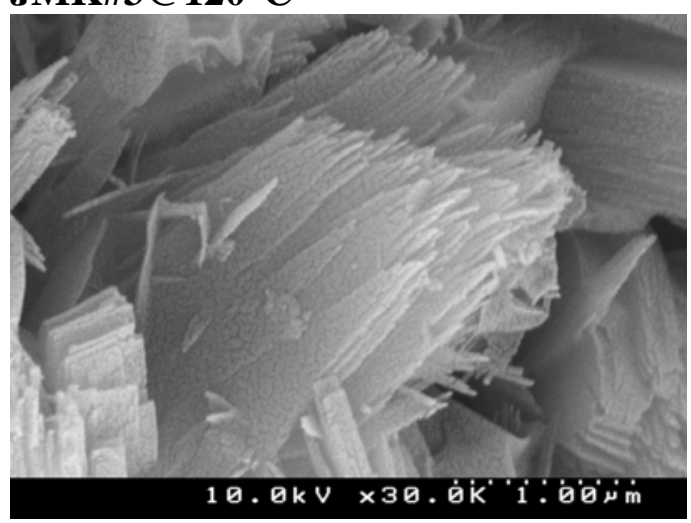

JMK\#3@200Ca

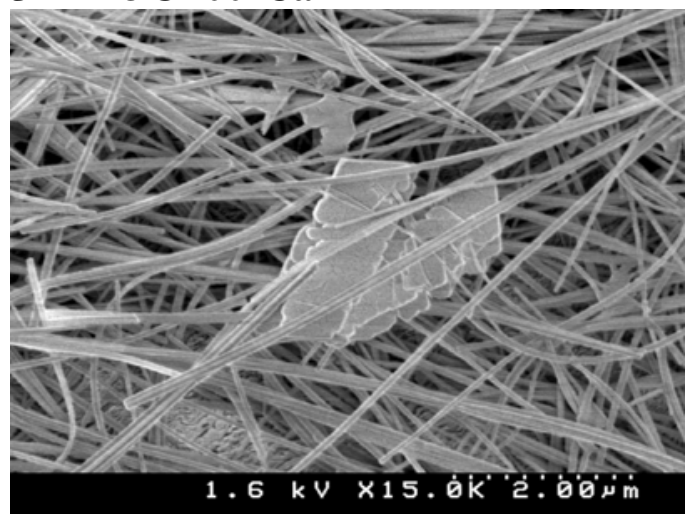

JMK\#3@120C

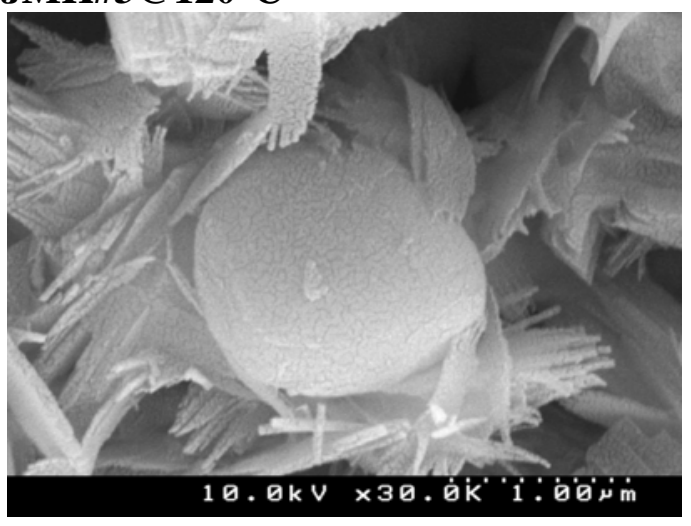

\section{JMK\#3@200Ca}

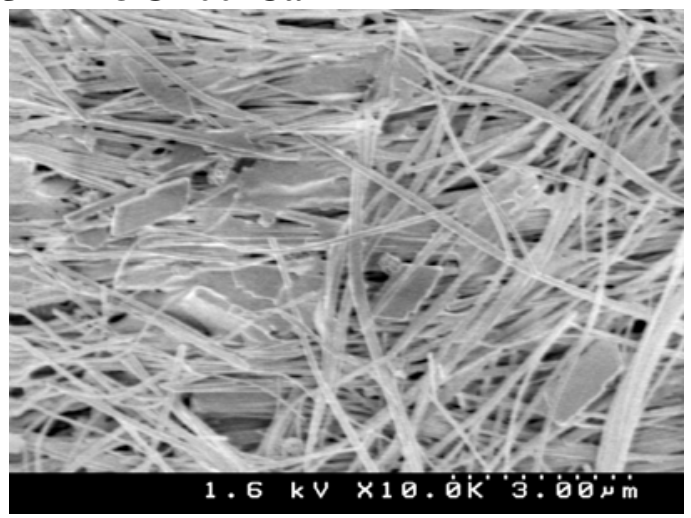

\section{JMK\#3@200Ca}

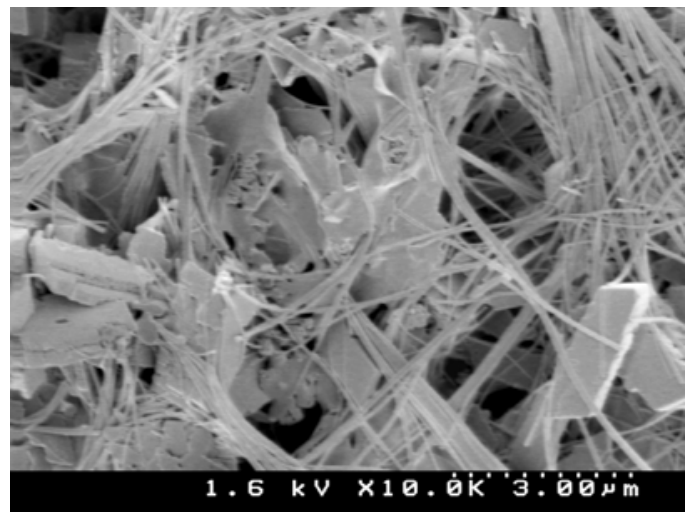


JMK\#3@200Ca

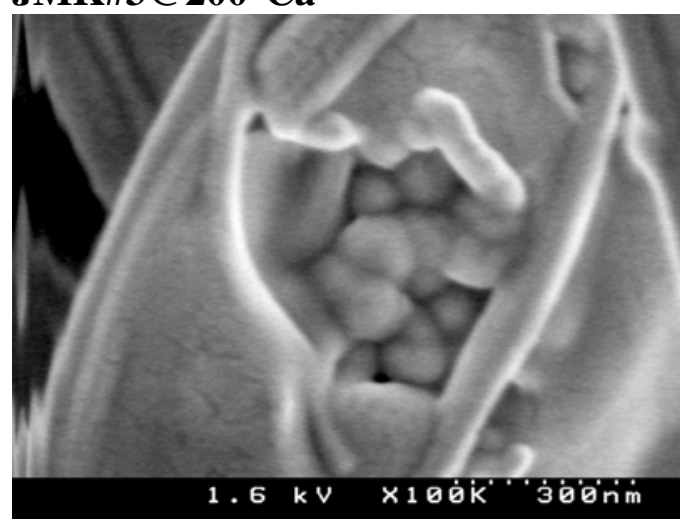

JMK\#3@200Ca

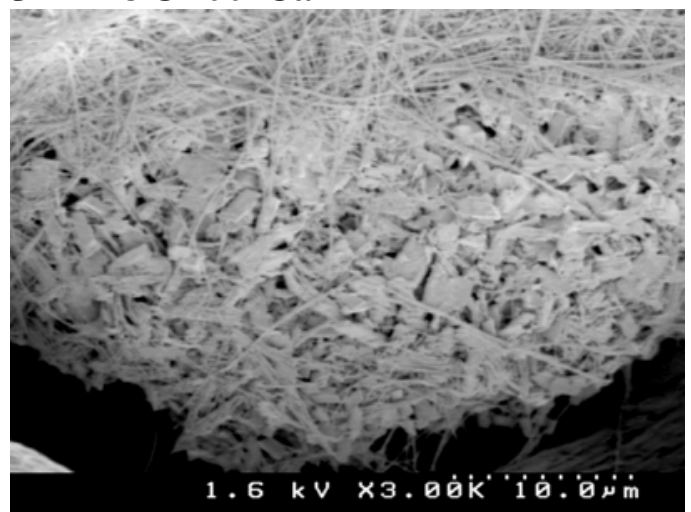

JMK\#3@200Ca

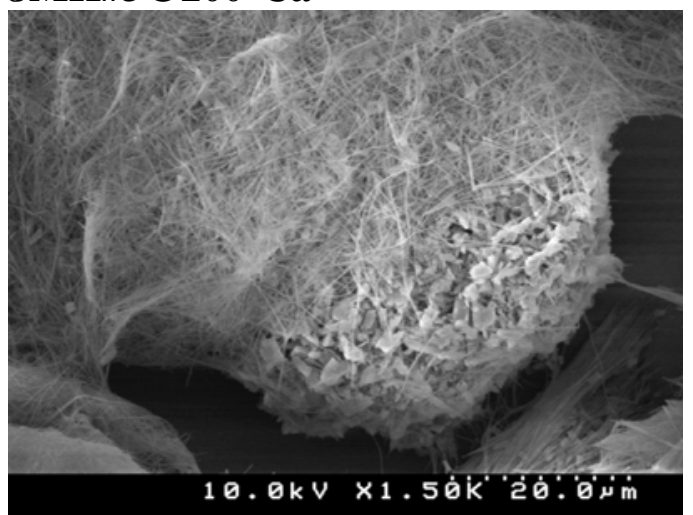

JMK\#3@200Ca

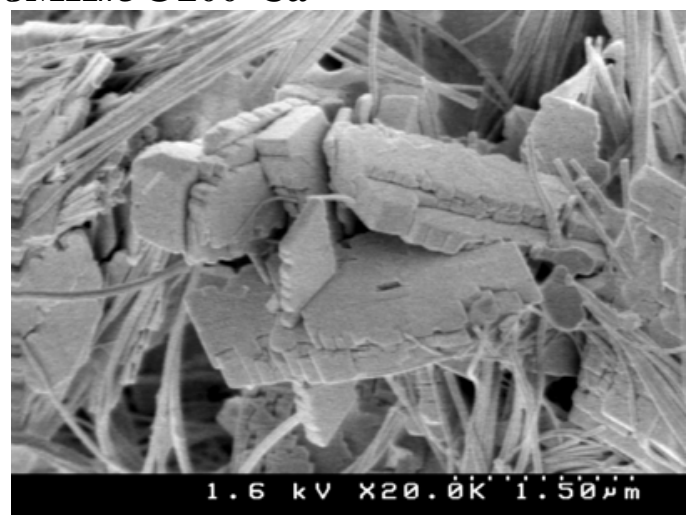

JMK\#3@200Ca

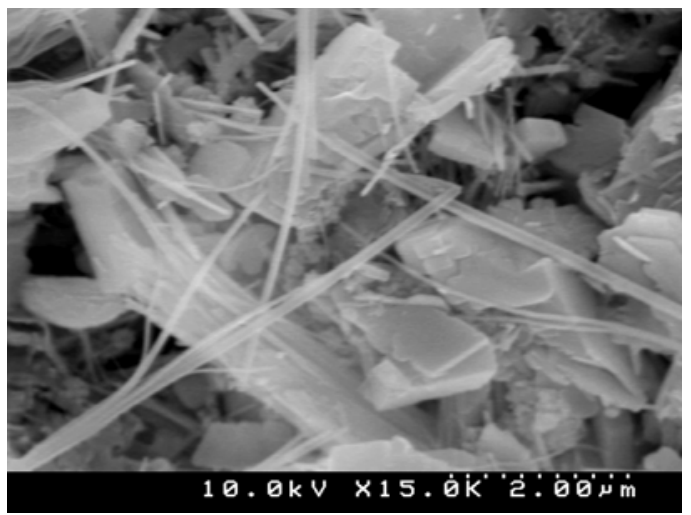

JMK\#3@200Ca

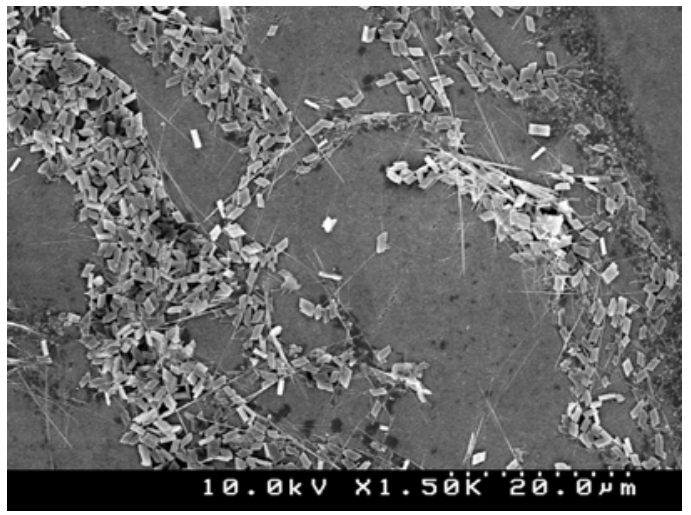


JMK\#3@200Ca

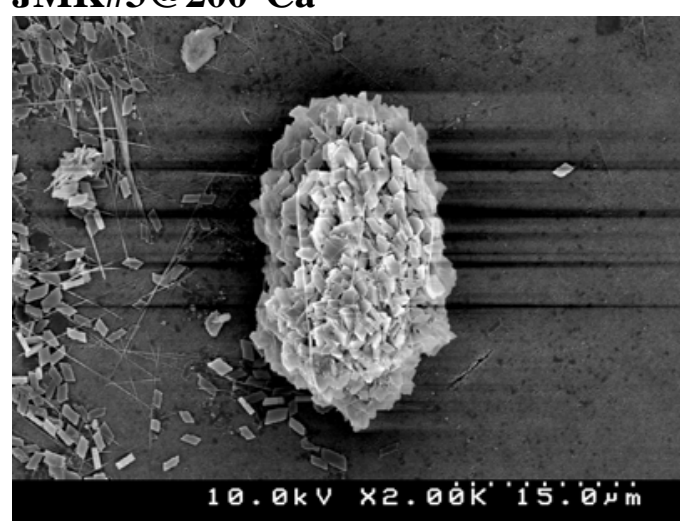

$10.0 \mathrm{kV} \times 2.0 \dot{\mathrm{k}} \mathrm{i} \mathrm{S}^{\circ} \mathrm{\theta}^{\mathrm{j}} \mathrm{m}$
JMK\#3@200Ca

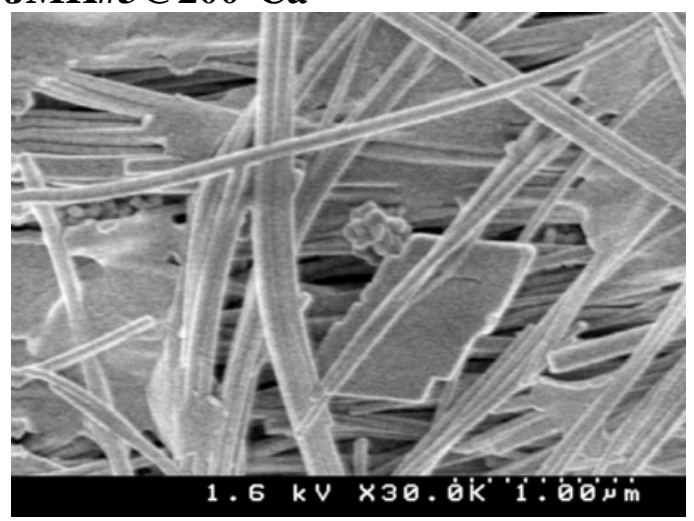

JMK\#3@200Cb

JMK\#3@200Cb
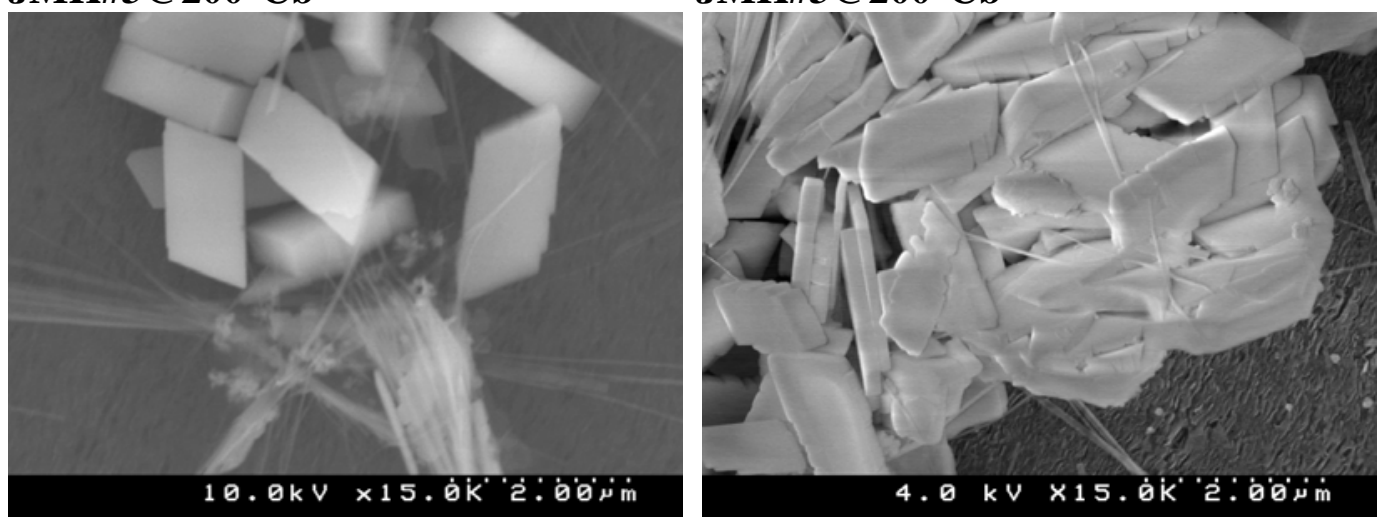

JMK\#3@200Cb

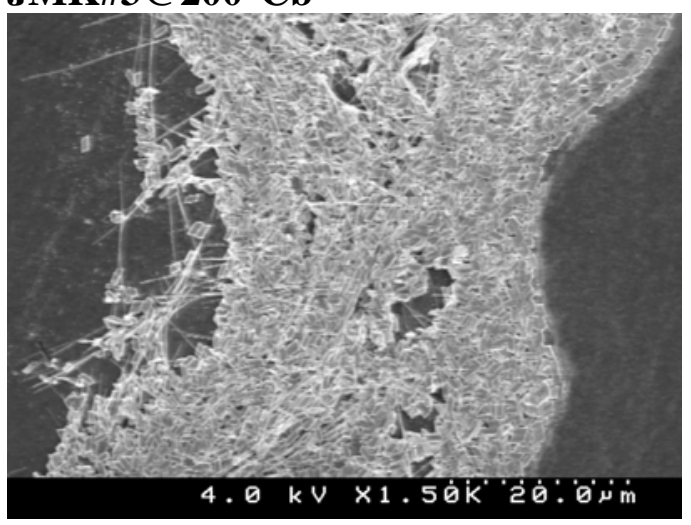




\title{
Chapter 5
}

\section{Stable Isotope Investigation of Precipitation and Recharge Processes in Nevada}

\author{
T.P. Rose \\ Chemical Biology and Nuclear Science Division \\ Lawrence Livermore National Laboratory
}

\subsection{Introduction}

This chapter summarizes the results of an on-going stable isotope study of precipitation and recharge processes in Nevada. Funding for this study is provided by the National Nuclear Security Administration, Nevada Site Office (NNSA/NSO) Hydrologic Resources Management Program (HRMP), and earlier descriptions of this work are found in the FY2001-2002 and FY2003 HRMP-UGTA progress reports (Rose et al., 2003; 2004).

This study consists of two separate but interrelated activities:

(1) Precipitation, spring water, and soil water samples were collected from four sites in central Nevada starting in 1999. Data acquired from these sites is used to determine the average stable isotope $\left(\delta \mathrm{D}, \delta^{18} \mathrm{O}\right)$ composition of precipitation, the relative contribution of cool and warm season precipitation to the recharge budget, and the timing and extent of evaporation during recharge. The sites were sampled biannually up through May 2004, and once each during 2005 and 2006. In the fall of 2006, the Desert Research Institute (DRI) took over sample collection responsibilities for these sites, and a return to a bi-annual sampling schedule is planned. LLNL will continue to perform stable isotope measurements on future samples from these sites.

(2) Precipitation samples were also collected on a biannual basis at five High Altitude Precipitation (HAP) stations maintained by the U.S. Geological Survey (USGS) throughout east-central and southern Nevada. Samples from these sites are sent to LLNL for stable isotope analyses. The HAP sites are more widely distributed geographically compared to the LLNL sites (Figure 5.1) and located at higher elevations (Table 5.1). These sites therefore provide insight into isotopic variations in precipitation as a function of both latitude and altitude that would not be possible with only the LLNL sites. Although most of the HAP sites were established in the mid-1980s, the present stable isotope investigation was initiated in 2002, and good quality isotope data were first generated in 2003. 
Table 5.1 Locations of Precipitation Gauges and Springs

\begin{tabular}{lccc}
\hline \hline & $\begin{array}{c}\text { Latitude } \\
(\mathrm{d} \mathrm{m} \mathrm{s})\end{array}$ & $\begin{array}{c}\text { Longitude } \\
(\mathrm{d} \mathrm{m} \mathrm{s})\end{array}$ & $\begin{array}{c}\text { Elevation } \\
(\mathrm{m})\end{array}$ \\
\hline LLNL Sites & & & \\
$\begin{array}{l}\text { Austin Summit } \\
\quad \text { Precipitation gauge/lysimeter }\end{array}$ & 392853 & 1170222 & 2285 \\
$\quad$ Spring & 392851 & 1170239 & 2240 \\
$\begin{array}{l}\text { Pinto Summit } \\
\quad \text { Precipitation gauge/lysimeter }\end{array}$ & 392703 & 1155606 & 2255 \\
$\quad$ Spring & 392725 & 1155618 & 2240 \\
Little Antelope Summit & 392346 & 1152801 & 2267 \\
$\quad$ Precipitation gauge/lysimeter & 392457 & 1152935 & 2200 \\
$\quad$ Spring & 384905 & 1151658 & 2140 \\
Currant Summit & 384922 & 1151804 & 2085 \\
$\quad$ Precipitation gauge/lysimeter & & & \\
$\quad$ Spring & 391436 & 1153239 & 3230 \\
USGS HAP Sites & 385409 & 1141854 & 3180 \\
Mt. Hamilton & 381157 & 1153731 & 2775 \\
Mt. Washington & 380026 & 1162738 & 2775 \\
Quinn Canyon Range & 363929 & 1151158 & 2999 \\
Kawich Range & & & \\
Hayford Peak & & & \\
\hline \hline
\end{tabular}

\subsection{LLNL Monitoring Sites}

Precipitation gauges were installed in January 1999 at three locations along the Hwy 50 corridor (Austin Summit, Pinto Summit, and Little Antelope Summit) and one location along Hwy 6 (Currant Summit). Site locations are shown in Figure 5.1. The precipitation gauges consist of $1 \mathrm{~m}$ segments of $15.7 \mathrm{~cm}$ diameter PVC pipe, capped at one end and mounted vertically in a stone foundation. Evaporation effects are minimized by adding mineral oil to the bottom of the gauges. Site elevations range from 2140 to $2280 \mathrm{~m}$ above sea level. Between 1999 and 2004, integrated samples of cool and warm season precipitation were collected on a biannual basis, usually in May and October. Following the May 2004 sampling event, samples were only collected once a year in 2005 and 2006. The measured precipitation amounts and stable isotope $\left(\delta \mathrm{D}\right.$ and $\left.\delta^{18} \mathrm{O}\right)$ values for each sampling event are presented in Table 5.2. Mean precipitation amounts and weighted mean isotopic values for each site are summarized in Table 5.3. Weighted mean isotopic values are calculated from the expression:

$$
\delta \text { mean }=\frac{\sum \delta_{\mathrm{i}} x_{\mathrm{i}}}{\sum x_{\mathrm{i}}}
$$

where $\delta_{\mathrm{i}}$ is the measured isotopic value for a given sample, and $x_{\mathrm{i}}$ is the corresponding precipitation amount (in $\mathrm{cm}$ ). 


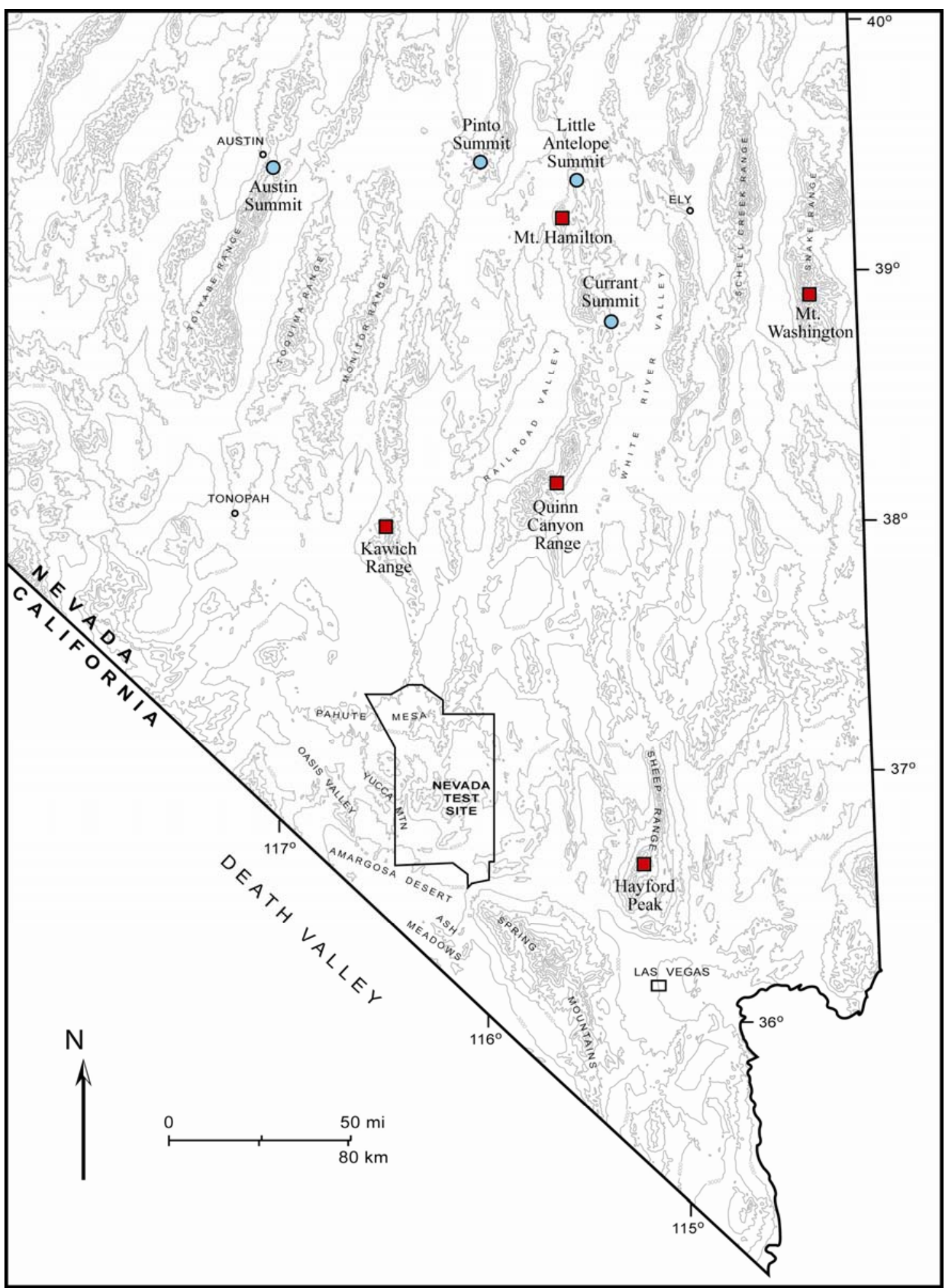

Figure 5.1 Map of southern and east-central Nevada showing the locations of sampling sites discussed in this report:

LLNL sites (circle); USGS HAP sites (square). 
Table 5.2 Precipitation Data for LLNL Sites in Central Nevada

\begin{tabular}{|c|c|c|c|c|}
\hline $\begin{array}{l}\text { Location / } \\
\text { Sample Date }\end{array}$ & $\begin{array}{l}\text { Time } \\
\text { Period }\end{array}$ & $\begin{array}{l}\text { Precipitation } \\
\text { Amount }(\mathrm{cm})\end{array}$ & $\begin{array}{l}\delta^{18} \mathrm{O} \\
(\% 0)\end{array}$ & $\begin{array}{l}\delta \mathrm{D} \\
(\% \circ)\end{array}$ \\
\hline \multicolumn{5}{|c|}{ Austin Summit } \\
\hline $\begin{array}{l}\text { 16-Jul-99 } \\
1 \text {-Nov-99 } \\
15-M a y-00 \\
16-O c t-00 \\
14-M a y-01 \\
24-O c t-01 \\
22-M a y-02 \\
9-\text { Oct-02 } \\
23-M a y-03 \\
11-O c t-03 \\
22-M a y-04 \\
21-M a y-05 \\
19-M a y-06\end{array}$ & $\begin{array}{l}01 / 99-07 / 99 \\
07 / 99-10 / 99 \\
11 / 99-05 / 00 \\
05 / 00-10 / 00 \\
10 / 00-05 / 01 \\
05 / 01-10 / 01 \\
10 / 01-05 / 02 \\
05 / 02-10 / 02 \\
10 / 02-05 / 03 \\
05 / 03-10 / 03 \\
10 / 03-05 / 04 \\
05 / 04-05 / 05 \\
05 / 05-05 / 06\end{array}$ & $\begin{array}{c}13.7 \\
0.7 \\
11.0 \\
7.1 \\
12.1 \\
2.1 \\
17.3 \\
3.2 \\
18.1 \\
8.2 \\
12.3 \\
34.5 \\
---\end{array}$ & $\begin{array}{c}-14.7 \\
-5.9 \\
-17.1 \\
-12.0 \\
-15.8 \\
-6.2 \\
-14.6 \\
-8.9 \\
-15.1 \\
-5.7 \\
-15.6 \\
-15.5 \\
---\end{array}$ & $\begin{array}{l}-107 \\
-41 \\
-131 \\
-92 \\
-116 \\
-39 \\
-105 \\
-61 \\
-110 \\
-38 \\
-116 \\
-115 \\
---\end{array}$ \\
\hline \multicolumn{5}{|l|}{ Pinto Summit } \\
\hline $\begin{array}{l}\text { 16-Jul-99 } \\
2-\text { Nov-99 } \\
15-M a y-00 \\
16-O c t-00 \\
14-M a y-01 \\
24-O c t-01 \\
23-M a y-02 \\
9-O c t-02 \\
23-M a y-03 \\
12-O c t-03 \\
22-M a y-04 \\
21-M a y-05 \\
19-M a y-06\end{array}$ & $\begin{array}{l}01 / 99-07 / 99 \\
07 / 99-10 / 99 \\
11 / 99-05 / 00 \\
05 / 00-10 / 00 \\
10 / 00-05 / 01 \\
05 / 01-10 / 01 \\
10 / 01-05 / 02 \\
05 / 02-10 / 02 \\
10 / 02-05 / 03 \\
05 / 03-10 / 03 \\
10 / 03-05 / 04 \\
05 / 04-05 / 05 \\
05 / 05-05 / 06\end{array}$ & $\begin{array}{c}20.7 \\
2.6 \\
19.5 \\
12.6 \\
25.9 \\
3.9 \\
23.4 \\
6.4 \\
27.8 \\
3.7 \\
17.1 \\
52.7 \\
35.4\end{array}$ & $\begin{array}{l}-15.9 \\
-8.3 \\
-16.1 \\
-12.3 \\
-16.7 \\
-6.6 \\
-15.2 \\
-11.0 \\
-16.0 \\
-5.5 \\
-16.4 \\
-15.7 \\
-13.9\end{array}$ & $\begin{array}{l}-119 \\
-50 \\
-117 \\
-89 \\
-121 \\
-43 \\
-111 \\
-79 \\
-118 \\
-36 \\
-123 \\
-115 \\
-99\end{array}$ \\
\hline \multicolumn{5}{|c|}{ Little Antelope Summit } \\
\hline $\begin{array}{l}\text { 16-Jul-99 } \\
2 \text {-Nov-99 } \\
16-M a y-00 \\
16-O c t-00 \\
14-M a y-01 \\
24-O c t-01 \\
23-M a y-02 \\
9-O c t-02 \\
23-M a y-03 \\
12-O c t-03 \\
22-M a y-04 \\
21-M a y-05 \\
19-M a y-06\end{array}$ & $\begin{array}{l}01 / 99-07 / 99 \\
07 / 99-10 / 99 \\
11 / 99-05 / 00 \\
05 / 00-10 / 00 \\
10 / 00-05 / 01 \\
05 / 01-10 / 01 \\
10 / 01-05 / 02 \\
05 / 02-10 / 02 \\
10 / 02-05 / 03 \\
05 / 03-10 / 03 \\
10 / 03-05 / 04 \\
05 / 04-05 / 05 \\
05 / 05-05 / 06\end{array}$ & $\begin{array}{c}21.4 \\
6.1 \\
17.2 \\
9.0 \\
23.4 \\
5.1 \\
23.6 \\
5.5 \\
24.4 \\
7.8 \\
20.9 \\
50.4 \\
32.3\end{array}$ & $\begin{array}{l}-15.7 \\
-6.7 \\
-17.4 \\
-10.6 \\
-17.6 \\
-5.6 \\
-15.2 \\
-15.1 \\
-15.7 \\
-7.8 \\
-16.6 \\
-15.3 \\
-14.6\end{array}$ & $\begin{array}{l}-116 \\
-37 \\
-128 \\
-75 \\
-131 \\
-35 \\
-111 \\
-113 \\
-116 \\
-50 \\
-124 \\
-110 \\
-106\end{array}$ \\
\hline \multicolumn{5}{|c|}{ Currant Summit } \\
\hline $\begin{array}{l}\text { 17-Jul-99 } \\
\text { 3-Nov-99 } \\
16-\text { May-00 } \\
\text { 17-Oct-00 } \\
\text { 15-May-01 } \\
24-O c t-01 \\
23-M a y-02 \\
10-O c t-02 \\
22-\text { May-03 } \\
\text { 13-Oct-03 } \\
\text { 23-May-04 } \\
\text { 22-May-05 } \\
\text { 20-May-06 }\end{array}$ & $\begin{array}{l}01 / 99-07 / 99 \\
07 / 99-10 / 99 \\
11 / 99-05 / 00 \\
05 / 00-10 / 00 \\
10 / 00-05 / 01 \\
05 / 01-10 / 01 \\
10 / 01-05 / 02 \\
05 / 02-10 / 02 \\
10 / 02-05 / 03 \\
05 / 03-10 / 03 \\
10 / 03-05 / 04 \\
05 / 04-05 / 05 \\
05 / 05-05 / 06\end{array}$ & $\begin{array}{c}--- \\
3.1 \\
16.7 \\
7.2 \\
21.4 \\
5.5 \\
12.7 \\
4.1 \\
17.6 \\
2.9 \\
16.5 \\
40.0 \\
23.1\end{array}$ & $\begin{array}{c}--- \\
-6.6 \\
-16.1 \\
-9.4 \\
-16.6 \\
-5.8 \\
-16.5 \\
-11.9 \\
-15.6 \\
-6.8 \\
-14.5 \\
-15.2 \\
-13.5\end{array}$ & $\begin{array}{c}--- \\
-41 \\
-118 \\
-66 \\
-120 \\
-36 \\
-121 \\
-86 \\
-115 \\
-48 \\
-106 \\
-110 \\
-94\end{array}$ \\
\hline
\end{tabular}

Dashed line indicates no sample was collected (due to vandalism). 


\section{Table 5.3 Mean Precipitation Amounts and Isotopic Values for LLNL Sites in Central Nevada}

\begin{tabular}{|c|c|c|c|c|c|c|c|c|}
\hline & \multicolumn{2}{|c|}{$\begin{array}{l}\text { Austin } \\
\text { Summit }\end{array}$} & \multicolumn{2}{|c|}{$\begin{array}{l}\text { Pinto } \\
\text { Summit }\end{array}$} & \multicolumn{2}{|c|}{$\begin{array}{l}\text { Little Antelope } \\
\text { Summit }\end{array}$} & \multicolumn{2}{|c|}{$\begin{array}{l}\text { Currant } \\
\text { Summit }\end{array}$} \\
\hline & \multicolumn{8}{|c|}{ Mean Precipitation Amounts } \\
\hline & \multicolumn{2}{|c|}{$(\mathrm{cm})$} & \multicolumn{2}{|c|}{$(\mathrm{cm})$} & \multicolumn{2}{|c|}{$(\mathrm{cm})$} & \multicolumn{2}{|c|}{$(\mathrm{cm})$} \\
\hline $\begin{array}{l}\text { Annual } \\
\text { Warm Season } \\
\text { Cool Season }\end{array}$ & \multicolumn{2}{|c|}{$\begin{array}{c}4.3 \\
14.2\end{array}$} & \multicolumn{2}{|c|}{$\begin{array}{c}5.8 \\
22.7\end{array}$} & \multicolumn{2}{|c|}{$\begin{array}{c}6.7 \\
21.9\end{array}$} & & \\
\hline & \multicolumn{8}{|c|}{ Weighted Mean Isotopic Values } \\
\hline & $\begin{array}{l}\delta^{18} \mathrm{O} \\
(\%)\end{array}$ & $\begin{array}{c}\delta \mathrm{D} \\
(\%)\end{array}$ & $\begin{array}{l}\delta^{18} \mathrm{O} \\
(\% 0)\end{array}$ & $\begin{array}{l}\delta \mathrm{D} \\
(\%)\end{array}$ & $\begin{array}{l}\delta^{18} \mathrm{O} \\
(\%)\end{array}$ & $\begin{array}{l}\delta \mathrm{D} \\
(\%)\end{array}$ & $\begin{array}{l}\delta^{18} \mathrm{O} \\
(\%)\end{array}$ & $\begin{array}{l}\delta \mathrm{D} \\
(\%)\end{array}$ \\
\hline $\begin{array}{l}\text { Full Record } \\
\text { Warm Season } \\
\text { Cool Season }\end{array}$ & $\begin{array}{c}-14.3 \\
-8.3 \\
-15.5\end{array}$ & $\begin{array}{c}-105 \\
-60 \\
-114\end{array}$ & $\begin{array}{l}-15.0 \\
-10.0 \\
-16.1\end{array}$ & $\begin{array}{c}-109 \\
-70 \\
-118\end{array}$ & $\begin{array}{c}-14.9 \\
-9.2 \\
-16.4\end{array}$ & $\begin{array}{c}-109 \\
-62 \\
-122\end{array}$ & $\begin{array}{l}-14.4 \\
-8.3 \\
-15.9\end{array}$ & $\begin{array}{l}-104 \\
-57 \\
-116\end{array}$ \\
\hline
\end{tabular}

Mean precipitation amounts range from $21.1 \mathrm{~cm} / \mathrm{yr}$ at Austin Summit to $33.0 \mathrm{~cm} / \mathrm{yr}$ at Pinto Summit, measured over a seven year period from July 1999 to May 2006. Seasonal data collected over a shorter period (1999-2004) reveal that only 20 to $23 \%$ of the annual precipitation occurs during the warm season (May to October), with the remaining 77 to $80 \%$ of the precipitation falling during the cool winter months. Note that the sum of the cool + warm season means is less than the annual means at all four sites (Table 5.3). This reflects the difference in sample collection periods (five years for seasonal data vs. seven years for annual data). The period from May 2004 to May 2005 was unusually wet, with annual precipitation rates between 156 and $164 \%$ of the mean values. These data were included in the annual means but not the seasonal means, which helps to explain why the former exceeds the sum of the latter.

The weighted mean $\delta \mathrm{D}$ and $\delta^{18} \mathrm{O}$ values for seasonal precipitation samples show the expected enrichment in heavy isotopes (less negative values) during the warm season and depletion in heavy isotopes (more negative values) during the cool season, in accordance with the temperature dependence of isotopic fractionation between vapor and condensed phases (e.g. Criss, 1999). The annual mean $\delta \mathrm{D}$ and $\delta^{18} \mathrm{O}$ values are strongly skewed toward the cool season average, which is consistent with the fact that $\sim 80 \%$ of all 
precipitation occurs during the cool season. Although the four sites are up to $150 \mathrm{~km}$ apart, the annual mean isotope values are remarkably similar at all locations, varying by only $0.7 \%$ in $\delta^{18} \mathrm{O}$ and $5 \%$ in $\delta \mathrm{D}$. This may reflect the tendency for winter low-pressure systems to be regional in extent.

To investigate local recharge processes, both soil water and spring water samples were collected from each monitoring site. Soil water samples were acquired over five winter seasons (2000-2004) using small lysimeters that were buried near the precipitation gauges. The lysimeter consisted of a $15.7 \mathrm{~cm}$ diameter funnel attached to a 2L-HDPE bottle with silicone sealant and wrapped with watertight tape along the seal. A $6 \mathrm{~mm}$ OD copper tube (approximately $0.5 \mathrm{~m}$ in length) is inserted through a separate hole at the top of the bottle to maintain a constant pressure between the interior of the bottle and the atmosphere. Mineral oil was added to the bottom of the lysimeter to prevent evaporation, and the mouth of the funnel was filled with smooth pebbles to facilitate drainage of water from the top of the funnel into the bottle. The lysimeter assembly was then buried with the top of the funnel at $\sim 10 \mathrm{~cm}$ below the soil surface. The soil layer above the lysimeter was carefully replaced, loosely packed and then leveled to avoid forming a depression.

The lysimeter samples provide valuable information on the isotopic composition and amount of fluid that infiltrated the upper soil zone at a point location. The data are summarized in Table 5.4. Only one lysimeter was used at each site from 2000 to 2002, whereas two lysimeters were used in 2003 and 2004. The two collectors were installed at identical depths $(10 \mathrm{~cm})$ one to three meters apart. The data acquired in 2003 and 2004 shows that the volume and composition of the soil water can vary substantially at a single site. These variations may reflect localized differences in soil compaction, snow pack depth, and snowmelt infiltration rates. The $\delta \mathrm{D}$ and $\delta^{18} \mathrm{O}$ values of the soil water samples also provide insight into how much evaporation occurred as the fluid moved through the uppermost soil zone. Evaporated waters always fall to the right of the global meteoric water line $\left(\delta \mathrm{D}=8 \delta^{18} \mathrm{O}+10\right.$; Craig, 1961) on a plot of $\delta \mathrm{D}$ versus $\delta^{18} \mathrm{O}$ (see discussion below). In general, decreasing fluid infiltration volumes are correlated with increasing amounts of evaporation. Conversely, when the amount of soil water infiltration is 'large' (greater than about $1 \mathrm{~cm}$ rainfall equivalent) the composition of the fluid tends to plot along the meteoric water line. The lack of evaporation in the larger volume samples may indicate that infiltration occurred over a relatively brief period of time, such as during a rapid snow melt event.

Perched springs located near each gauging site are an additional source of comparative data. In contrast to the lysimeters, the perched springs represent the integrated $\delta \mathrm{D}$ and $\delta^{18} \mathrm{O}$ composition of groundwater recharge over a much larger area. Discharge rate and water quality parameters (temperature, conductivity, alkalinity, and $\mathrm{pH}$ ) were also measured at the spring sites. The data are summarized in Table 5.5. The spring waters at all four sites showed consistent isotopic compositions over time, though the discharge rates at three of the four sites sometimes varied by more than an order of magnitude. Water quality parameters also tended to vary over time. The slightly mineralized spring located near Little Antelope Summit showed the most consistent set of chemical, isotopic and discharge characteristics of the four springs. 
Table 5.4 Lysimeter Data for LLNL Sites in Central Nevada

\begin{tabular}{|c|c|c|c|c|c|}
\hline $\begin{array}{l}\text { Location / } \\
\text { Sample Date }\end{array}$ & $\begin{array}{l}\text { Time } \\
\text { Period }\end{array}$ & $\begin{array}{l}\text { Infiltration } \\
\text { Amount } \\
(\mathrm{cm})\end{array}$ & $\begin{array}{l}\text { Infiltration / } \\
\text { Precipitation } \\
\text { (ratio) }\end{array}$ & $\begin{array}{l}\delta^{18} \mathrm{O} \\
(\% 0)\end{array}$ & $\begin{array}{l}\delta \mathrm{D} \\
(\% 0)\end{array}$ \\
\hline \multicolumn{6}{|l|}{ Austin Summit } \\
\hline 15-May-00 & $11 / 99-05 / 00$ & 0.2 & 0.02 & -16.2 & -132 \\
\hline 14-May-01 & $10 / 00-05 / 01$ & 0.7 & 0.06 & -16.9 & -124 \\
\hline 22-May-02 & $10 / 01-05 / 02$ & 10.3 & 0.59 & -14.5 & -105 \\
\hline $\begin{array}{l}\text { 23-May-03 (\#1) } \\
\text { 23-May-03 (\#2) }\end{array}$ & $\begin{array}{l}10 / 02-05 / 03 \\
10 / 02-05 / 03\end{array}$ & $\begin{array}{c}\text { sample lost } \\
3.7\end{array}$ & $\overline{0 .-20}$ & $-\overline{-16.4}$ & $-\overline{--}$ \\
\hline $\begin{array}{l}\text { 22-May-04 (\#1) } \\
\text { 22-May-04 (\#2) }\end{array}$ & $\begin{array}{l}10 / 03-05 / 04 \\
10 / 03-05 / 04\end{array}$ & $\begin{array}{l}8.1 \\
4.0\end{array}$ & $\begin{array}{l}0.66 \\
0.33\end{array}$ & $\begin{array}{l}-17.6 \\
-17.1\end{array}$ & $\begin{array}{l}-133 \\
-129\end{array}$ \\
\hline \multicolumn{6}{|l|}{ Pinto Summit } \\
\hline 15-May-00 & $11 / 99-05 / 00$ & $<0.1$ & $<0.01$ & -12.6 & -101 \\
\hline 14-May-01 & $10 / 00-05 / 01$ & 10.4 & 0.40 & -15.6 & -115 \\
\hline 23-May-02 & $10 / 01-05 / 02$ & $<0.1$ & $<0.01$ & --- & --- \\
\hline $\begin{array}{l}\text { 23-May-03 (\#1) } \\
\text { 23-May-03 (\#2) }\end{array}$ & $\begin{array}{l}10 / 02-05 / 03 \\
10 / 02-05 / 03\end{array}$ & $\begin{array}{l}<0.1 \\
0.1\end{array}$ & $\begin{array}{l}<0.01 \\
<0.01\end{array}$ & $\begin{array}{l}-15.1 \\
-18.6\end{array}$ & $\begin{array}{l}-119 \\
-144\end{array}$ \\
\hline $\begin{array}{l}\text { 22-May-04 (\#1) } \\
\text { 22-May-04 (\#2) }\end{array}$ & $\begin{array}{l}10 / 03-05 / 04 \\
10 / 03-05 / 04\end{array}$ & $\begin{array}{c}7.2 \\
10.2\end{array}$ & $\begin{array}{l}0.42 \\
0.60\end{array}$ & $\begin{array}{l}-17.3 \\
-17.8\end{array}$ & $\begin{array}{l}-129 \\
-132\end{array}$ \\
\hline \multicolumn{6}{|c|}{ Little Antelope Summit } \\
\hline 16-May-00 & $11 / 99-05 / 00$ & $<0.1$ & $<0.01$ & --- & --- \\
\hline 14-May-01 & $10 / 00-05 / 01$ & 0.1 & $<0.01$ & -18.3 & -130 \\
\hline 23-May-02 & $10 / 01-05 / 02$ & 0.2 & 0.01 & -15.2 & -119 \\
\hline $\begin{array}{l}\text { 23-May-03 (\#1) } \\
\text { 23-May-03 (\#2) }\end{array}$ & $\begin{array}{l}10 / 02-05 / 03 \\
10 / 02-05 / 03\end{array}$ & $\begin{array}{l}6.8 \\
1.1\end{array}$ & $\begin{array}{l}0.37 \\
0.04\end{array}$ & $\begin{array}{l}-18.3 \\
-17.7\end{array}$ & $\begin{array}{l}-137 \\
-134\end{array}$ \\
\hline $\begin{array}{l}\text { 22-May-04 (\#1) } \\
\text { 22-May-04 (\#2) }\end{array}$ & $\begin{array}{l}10 / 03-05 / 04 \\
10 / 03-05 / 04\end{array}$ & $\begin{array}{l}1.4 \\
1.9\end{array}$ & $\begin{array}{l}0.07 \\
0.09\end{array}$ & $\begin{array}{l}-16.1 \\
-15.9\end{array}$ & $\begin{array}{l}-118 \\
-125\end{array}$ \\
\hline \multicolumn{6}{|l|}{ Currant Summit } \\
\hline 16-May-00 & $11 / 99-05 / 00$ & 2.8 & 0.17 & -16.2 & -120 \\
\hline 15-May-01 & $10 / 00-05 / 01$ & 1.2 & 0.06 & -15.3 & -112 \\
\hline 23-May-02 & $10 / 01-05 / 02$ & 0.5 & 0.04 & -13.4 & -116 \\
\hline $\begin{array}{l}\text { 22-May-03 (\#1) } \\
\text { 22-May-03 (\#2) }\end{array}$ & $\begin{array}{l}10 / 02-05 / 03 \\
10 / 02-05 / 03\end{array}$ & $\begin{array}{l}0.9 \\
0.2\end{array}$ & $\begin{array}{l}0.07 \\
0.01\end{array}$ & $\begin{array}{l}-11.2 \\
-10.9\end{array}$ & $\begin{array}{c}-101 \\
-89\end{array}$ \\
\hline $\begin{array}{l}\text { 23-May-04 (\#1) } \\
\text { 23-May-04 (\#2) }\end{array}$ & $\begin{array}{l}10 / 03-05 / 04 \\
10 / 03-05 / 04\end{array}$ & $\begin{array}{l}0.9 \\
1.9\end{array}$ & $\begin{array}{l}0.05 \\
0.11\end{array}$ & $\begin{array}{l}-12.6 \\
-10.9\end{array}$ & $\begin{array}{l}-97 \\
-79\end{array}$ \\
\hline
\end{tabular}


Table 5.5 Spring Water Data for LLNL Sites in Central Nevada

\begin{tabular}{|c|c|c|c|c|c|c|c|}
\hline $\begin{array}{l}\text { Location / } \\
\text { Sample Date }\end{array}$ & $\begin{array}{c}\text { Est. Flow } \\
\text { Rate (L/min) }\end{array}$ & $\begin{array}{l}\text { Temp. } \\
\left({ }^{\circ} \mathrm{C}\right)\end{array}$ & $\mathrm{pH}$ & $\begin{array}{l}\text { Cond. } \\
(\mu \mathrm{S} / \mathrm{cm})\end{array}$ & $\begin{array}{l}\text { Alkalinity } \\
(\mathrm{mg} / \mathrm{L})\end{array}$ & $\begin{array}{l}\delta^{18} O \\
(\% \circ)\end{array}$ & $\begin{array}{c}\delta \mathrm{D} \\
(\% \mathrm{o})\end{array}$ \\
\hline \multicolumn{8}{|c|}{ Austin Summit Spring } \\
\hline $\begin{array}{l}\text { 1-Nov-99 } \\
\text { 15-May-00 } \\
\text { 16-Oct-00 } \\
\text { 14-May-01 } \\
\text { 24-Oct-01 } \\
\text { 22-May-02 } \\
\text { 9-Oct-02 } \\
\text { 23-May-03 } \\
\text { 11-Oct-03 } \\
\text { 22-May-04 } \\
\text { 21-May-05 } \\
\text { 19-May-06 }\end{array}$ & $\begin{array}{c}1.0 \\
0.2 \\
0.1 \\
<0.1 \\
<0.1 \\
0.3 \\
1.5 \\
\text { dry } \\
0.1 \\
0.5 \\
4.5 \\
6.0\end{array}$ & $\begin{array}{c}9 \\
13 \\
6 \\
18 \\
7 \\
7 \\
10 \\
\text { n.d. } \\
11 \\
10 \\
9 \\
10\end{array}$ & $\begin{array}{l}7.83 \\
7.05 \\
7.20 \\
7.05 \\
7.80 \\
7.15 \\
7.35 \\
\text { n.d. } \\
8.10 \\
7.84 \\
6.96 \\
6.93\end{array}$ & $\begin{array}{l}239 \\
275 \\
315 \\
277 \\
377 \\
311 \\
333 \\
\text { n.d. } \\
301 \\
283 \\
348 \\
320\end{array}$ & $\begin{array}{l}125 \\
115 \\
\text { n.d. } \\
140 \\
150 \\
130 \\
\text { n.d. } \\
\text { n.d. } \\
115 \\
120 \\
120 \\
120\end{array}$ & $\begin{array}{r}-16.0 \\
-16.1 \\
-15.9 \\
-15.9 \\
-15.9 \\
-14.6 \\
-15.9 \\
\text { n.d. } \\
-16.0 \\
-16.1 \\
-16.1 \\
-16.3\end{array}$ & $\begin{array}{l}-118 \\
-117 \\
-116 \\
-118 \\
-120 \\
-107 \\
-122 \\
n . d . \\
-120 \\
-122 \\
-122 \\
-123\end{array}$ \\
\hline \multicolumn{8}{|c|}{ Pinto Summit Spring } \\
\hline $\begin{array}{l}\text { 2-Nov-99 } \\
\text { 15-May-00 } \\
\text { 16-Oct-00 } \\
\text { 14-May-01 } \\
\text { 24-Oct-01 } \\
\text { 23-May-02 } \\
\text { 9-Oct-02 } \\
\text { 23-May-03 } \\
\text { 12-Oct-03 } \\
\text { 22-May-04 } \\
\text { 21-May-05 } \\
\text { 19-May-06 }\end{array}$ & $\begin{array}{c}5 \\
15 \\
2 \\
3 \\
3 \\
6 \\
8 \\
10 \\
2 \\
6 \\
30 \\
8\end{array}$ & $\begin{array}{c}4 \\
7 \\
5 \\
12 \\
5 \\
15 \\
12 \\
18 \\
16 \\
14 \\
13 \\
14\end{array}$ & $\begin{array}{l}8.10 \\
7.50 \\
7.69 \\
8.02 \\
8.02 \\
7.50 \\
7.82 \\
8.36 \\
7.37 \\
7.28 \\
7.35 \\
7.55\end{array}$ & $\begin{array}{l}299 \\
395 \\
372 \\
373 \\
418 \\
460 \\
400 \\
368 \\
355 \\
382 \\
394 \\
439\end{array}$ & $\begin{array}{l}175 \\
150 \\
\text { n.d. } \\
185 \\
180 \\
175 \\
\text { n.d. } \\
200 \\
175 \\
200 \\
190 \\
225\end{array}$ & $\begin{array}{l}-15.2 \\
-15.7 \\
-15.4 \\
-15.4 \\
-15.2 \\
-15.1 \\
-15.5 \\
-15.7 \\
-15.3 \\
-15.4 \\
-15.8 \\
-15.6\end{array}$ & $\begin{array}{l}-120 \\
-120 \\
-122 \\
-121 \\
-119 \\
-121 \\
-121 \\
-122 \\
-121 \\
-121 \\
-122 \\
-118\end{array}$ \\
\hline \multicolumn{8}{|c|}{ Little Antelope Spring } \\
\hline $\begin{array}{l}\text { 2-Nov-99 } \\
\text { 16-May-00 } \\
\text { 16-Oct-00 } \\
\text { 14-May-01 } \\
\text { 24-Oct-01 } \\
\text { 23-May-02 } \\
\text { 9-Oct-02 } \\
\text { 23-May-03 } \\
\text { 12-Oct-03 } \\
\text { 22-May-04 } \\
\text { 21-May-05 } \\
\text { 19-May-06 }\end{array}$ & $\begin{array}{l}1.0 \\
1.0 \\
1.0 \\
0.9 \\
1.0 \\
0.8 \\
0.8 \\
0.75 \\
0.9 \\
0.2 \\
0.9 \\
1.2\end{array}$ & $\begin{array}{c}9 \\
8 \\
9 \\
8 \\
9 \\
8 \\
9 \\
9 \\
9 \\
8 \\
9 \\
10\end{array}$ & $\begin{array}{l}7.08 \\
6.98 \\
6.76 \\
6.95 \\
7.05 \\
7.05 \\
6.79 \\
7.10 \\
7.10 \\
7.53 \\
6.99 \\
7.46\end{array}$ & $\begin{array}{l}1421 \\
1661 \\
1665 \\
1684 \\
1520 \\
1521 \\
1601 \\
1630 \\
1672 \\
1590 \\
1738 \\
1735\end{array}$ & $\begin{array}{l}350 \\
400 \\
\text { n.d. } \\
350 \\
400 \\
400 \\
\text { n.d. } \\
350 \\
400 \\
350 \\
400 \\
500\end{array}$ & $\begin{array}{l}-15.5 \\
-15.7 \\
-15.6 \\
-15.6 \\
-15.6 \\
-15.6 \\
-15.6 \\
-15.6 \\
-15.5 \\
-15.7 \\
-15.8 \\
-15.8\end{array}$ & $\begin{array}{l}-122 \\
-122 \\
-124 \\
-125 \\
-126 \\
-125 \\
-125 \\
-123 \\
-124 \\
-123 \\
-125 \\
-124\end{array}$ \\
\hline \multicolumn{8}{|c|}{ Currant Summit Spring } \\
\hline $\begin{array}{l}\text { 3-Nov-99 } \\
\text { 16-May-00 } \\
\text { 17-Oct-00 } \\
\text { 15-May-01 } \\
\text { 24-Oct-01 } \\
\text { 23-May-02 } \\
\text { 10-Oct-02 } \\
\text { 22-May-03 } \\
\text { 13-Oct-03 } \\
\text { 23-May-04 } \\
\text { 22-May-05 } \\
\text { 20-May-06 }\end{array}$ & $\begin{array}{l}0.5 \\
0.1 \\
0.3 \\
1.2 \\
0.3 \\
0.1 \\
0.2 \\
0.03 \\
0.6 \\
0.03 \\
0.07 \\
0.2\end{array}$ & $\begin{array}{c}12 \\
2 \\
8 \\
13 \\
8 \\
7 \\
14 \\
25 \\
14 \\
19 \\
16 \\
14\end{array}$ & $\begin{array}{l}7.51 \\
7.20 \\
7.21 \\
7.72 \\
7.51 \\
7.52 \\
7.10 \\
8.41 \\
7.08 \\
7.90 \\
7.73 \\
7.43\end{array}$ & $\begin{array}{l}381 \\
477 \\
446 \\
456 \\
402 \\
388 \\
524 \\
513 \\
468 \\
420 \\
437 \\
438\end{array}$ & $\begin{array}{l}170 \\
160 \\
\text { n.d. } \\
160 \\
175 \\
175 \\
\text { n.d. } \\
175 \\
200 \\
160 \\
175 \\
180\end{array}$ & $\begin{array}{l}-13.9 \\
-13.9 \\
-14.0 \\
-14.0 \\
-13.9 \\
-13.9 \\
-13.9 \\
-13.8 \\
-13.9 \\
-13.9 \\
-14.1 \\
-14.2\end{array}$ & $\begin{array}{l}-108 \\
-110 \\
-112 \\
-112 \\
-111 \\
-109 \\
-110 \\
-109 \\
-111 \\
-109 \\
-109 \\
-111\end{array}$ \\
\hline
\end{tabular}

n.d. $=$ not determined 
Figure 5.2 shows $\delta \mathrm{D}$ vs. $\delta^{18} \mathrm{O}$ plots of weighted mean precipitation data (annual, cool, and warm season), weighted mean values for the lysimeter samples, and average spring water compositions for each monitoring site. The global meteoric water line is included for reference. All of the precipitation data plots very close to the meteoric water line, regardless of whether it was collected in the spring or fall. As noted above, the annual mean is much closer to the cool season average than the warm season average because most of the precipitation $(\sim 80 \%)$ occurs during the cool season.

Weighted mean isotopic values for soil waters (lysimeters) were calculated in the same manner as the weighted mean precipitation. If two soil water samples were collected at one site during the same year, the data were averaged before they were included in the weighted mean. The results provide some interesting insights into recharge processes. At three of the four locations, the soil water $\delta \mathrm{D}$ and $\delta^{18} \mathrm{O}$ values plot fairly close to the meteoric water line, and are depleted in heavy isotopes relative to the average cool season precipitation (see data points labeled 'lysimeter' in Figure 5.2). This result was somewhat unexpected but can be interpreted in the context of snow melt processes.

As a snow pack melts, the bulk isotopic composition of the residual snow becomes more enriched in heavy isotopes (Arnason et al., 1973; Stichler et al., 1981). This is because the fractionation of stable isotopes between coexisting water and ice favors enrichment of the solid phase in heavy isotopes (O'Neil, 1968). The isotopic enrichment in the residual snow must be balanced by losses of light isotopes, either as melt water or water vapor. Hermann et al. (1981) showed that light isotopes predominate during the initial stages of snow melt runoff, and that the heavy isotope content of the runoff steadily increases with time. Applying this information to the present study, we can infer that a majority of the melt water infiltrating the upper soil zone must be derived from the early stages of melting. It may be that less infiltration occurs during late stage melting because the soil becomes fully saturated, requiring greater amounts of surface runoff.

The Currant Summit data shows a pattern that differs from the other three sites. The average soil water has $\delta \mathrm{D}$ and $\delta^{18} \mathrm{O}$ values that are greater than the average cool season precipitation (Figure 5.2). In addition, the soil water ('lysimeter') data point is clearly shifted to the right of the meteoric water line, implying an evaporative isotopic enrichment process. On $\delta \mathrm{D}$ vs. $\delta^{18} \mathrm{O}$ plots, evaporated waters fall along linear trends with slopes between about 2 and 5 (Fontes, 1980). We can infer an initial (pre-evaporation) soil water composition by projecting an imaginary line from the lysimeter data point back to the meteoric water line. At Currant Summit, a relatively high slope $(\mathrm{m}=5)$ evaporation trajectory would yield a pre-evaporation composition similar to the cool season precipitation average - but no less than this composition. This may imply that a larger fraction of 'late stage' snow melt (which is more enriched in heavy isotopes) infiltrates the soil zone at this site. 

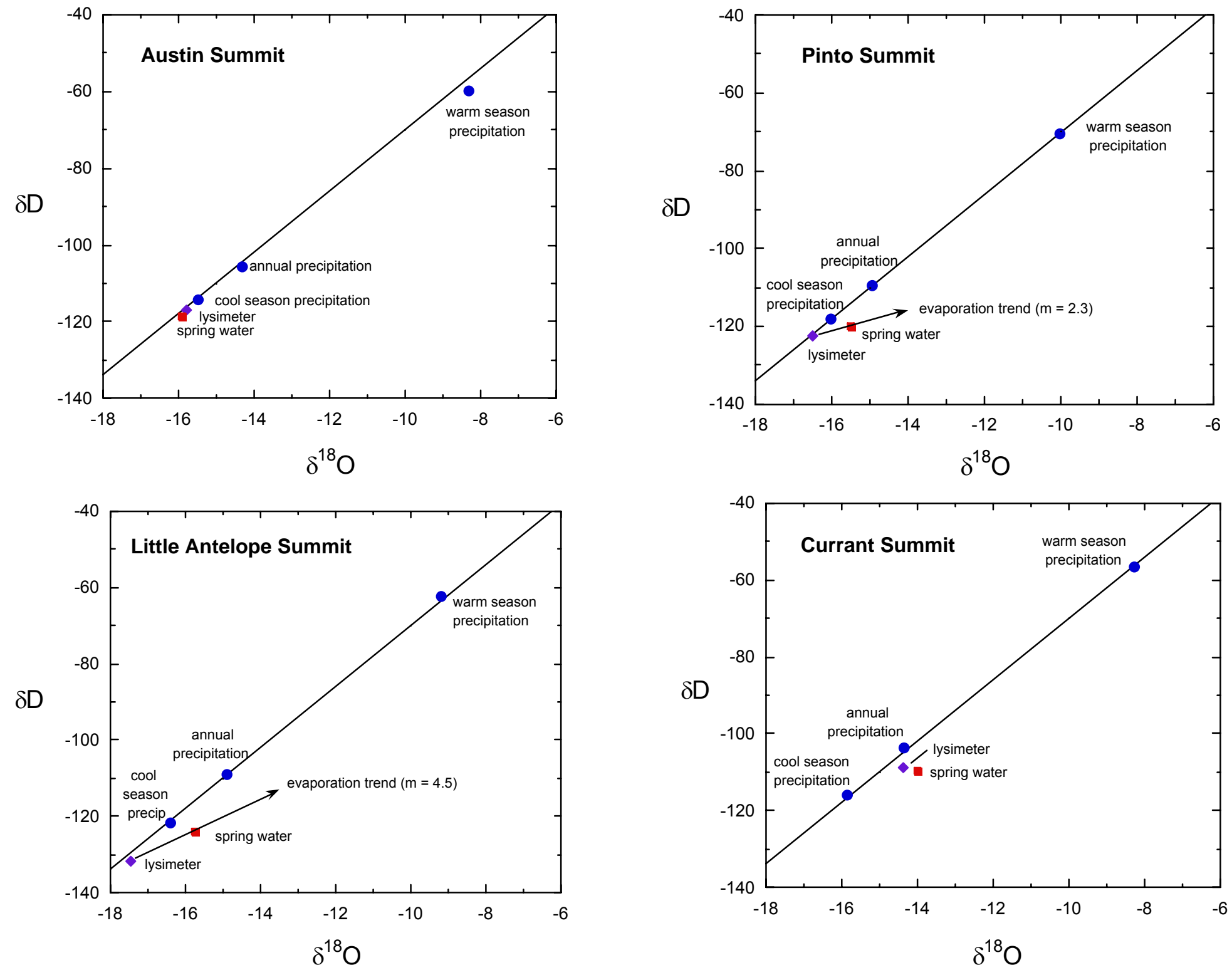

Figure 5.2 Plots of mean $\delta \mathrm{D}$ vs. $\delta^{18} \mathrm{O}$ values for water samples collected from LLNL monitoring sites in central Nevada. See text for discussion. 
There is no obvious explanation for why the soil water is more evaporated at Current Summit compared to the other study locations (Figure 5.2). A previous snow hydrology study reported kinetic isotope enrichments (similar to evaporation effects) in the Current Summit snow pack prior to melting (Rose et al., 1999). These enrichments were interpreted as evidence for water vapor loss during snow metamorphism. Although isotope enrichment prior to recharge is possible, it is unclear why such a process would occur at Current Summit and not at the other three sites. An alternative explanation is that the existing dataset does not accurately represent the time-integrated soil water isotopic composition at Currant Summit. A longer term data record may be required to obtain a meaningful average value.

Further insight into recharge processes is gained by comparing soil water (lysimeter) and spring water isotopic data. The spring water composition is more evaporated than the average soil water composition at three of the four sites (Figure 5.2). Austin Summit is the only site where almost no evaporation is observed (i.e., all data plot very near the meteoric water line). The average soil and spring water compositions are nearly identical at this location (Figure 2). The lack of evaporation may be due to a difference in soil properties relative to the other sites. The Austin Summit site is located on granitic bedrock, and the soil cover consists of a thin veneer of coarsely weathered quartz and feldspar grains through which rainfall and snow melt can pass quickly to reach the underlying fractured rock. In contrast, the bedrock at the other three sites consists of volcanic rock that weathers to form a comparatively fine-grained soil matrix.

The Pinto Summit and Little Antelope Summit datasets show very similar patterns in their average precipitation, soil water, and spring water compositions (Figure 2). In particular, the spring water at both sites can be reasonably derived by modest evaporation of the soil water (lysimeter) composition. Although the slope of the evaporation trend is different at the two sites (2.3 at Pinto vs. 4.5 at Little Antelope) both slopes are within the expected range of values (between 2 and 5). At Currant Summit, the soil and spring waters show similar degrees of evaporation and have similar $\delta \mathrm{D}$ and $\delta^{18} \mathrm{O}$ values, suggesting that both waters experienced a similar isotope enrichment pathway.

It is well known that spring waters are susceptible to evaporation after the water emerges from the spring orifice. During this study, post-discharge evaporation effects were minimized by collecting spring water samples directly from the point of discharge. For this reason, the evaporated isotopic signatures we observe at the perched springs are inferred to have been inherited during recharge. The conceptual model for this process is based on earlier studies of isotope profiles in soil moisture (e.g. Allison et al., 1983, Singleton et al., 2004). As the soil zone dries following a recharge event, the residual soil moisture gradually develops an evaporative stable isotope profile as a function of depth. With repeated episodes of wetting and drying, even the deeper soil moisture will evolve to a bulk isotopic composition that is evaporated relative to the initial soil moisture composition. Recharge events will push the evaporated soil moisture to the water table by piston flow. As a result, the bulk isotopic composition of the local water table (represented by perched springs) will be modestly evaporated relative to the initial 
isotopic composition of the water when it first enters the soil zone (represented by the lysimeter samples).

Large volume soil water samples collected in the lysimeters showed little or no evidence of evaporation because mineral oil was used to prevent the water from evaporating after it had entered the collector. In a natural system, this would not be the case. Conversely, whenever small volumes of fluid $(<1 \mathrm{~cm}$ rainfall equivalent) were collected in the lysimeters, the stable isotope signatures were always evaporated to some extent. These smaller volume samples probably reflect slower rates of soil moisture infiltration. In general, soil moisture evaporation is considered to be the most plausible mechanism to explain the evaporated isotope signatures of the perched springs.

\subsection{USGS High Altitude Precipitation (HAP) Sites}

The U.S. Geological Survey maintains a High Altitude Precipitation (HAP) monitoring network in eastern Nevada, within the Great Basin carbonate-rock province. Precipitation amounts are monitored on a bi-annual basis (spring and fall) and the data are published annually in USGS Water Resources Data reports (e.g. U.S. Geological Survey, 2006). The HAP network was originally established in the 1980s to support regional carbonate aquifer water-supply studies (e.g. Prudic et al., 1995; Thomas et al., 1996). Various aspects of these water resource investigations have continued to the present day (U.S. Geological Survey, 2005).

In 2002, LLNL staff began collaborating with the USGS office in Henderson, Nevada on the stable isotope analysis of samples from the HAP sites. During the first year of the project, the existing HAP gauges were used to collect samples. Unfortunately, various problems were encountered (see Rose et al., 2003) and it was necessary to abandon this approach. In June 2003, a new set of gauges referred to as 'bucket collectors' were installed at five HAP sites (see Table 5.1) including two new sites in the Kawich and Quinn Canyon Ranges. The first set of precipitation samples was retrieved from the bucket collectors in October 2003. These collectors yielded good quality stable isotope samples, and the sampling / analysis program has continued on a bi-annual basis since that time.

Analytical results for the USGS HAP samples are reported in Table 5.6. Precipitation amount data were provided by Tim Olson (USGS, Henderson). Although the data record is fairly short, it is instructive to calculate weighted mean $\delta \mathrm{D}$ and $\delta^{18} \mathrm{O}$ values for the annual, cool season, and warm season samples at each site (see Table 5.7). Note that the annual mean is very similar to the cool season mean, particularly at the northernmost sites (Mt. Washington and Mt. Hamilton). The heavy precipitation that occurred during the 2004-2005 winter season exerts a strong influence on the weighted mean values for all sites because of the short duration of the record. Note also that in the case of Mt. Washington, the warm season 'mean' is represented by only one sample. 
Weighted mean isotopic values are plotted in Figure 5.3 for cool and warm season samples from both the USGS HAP sites and the LLNL central Nevada sites. The two different datasets show overlapping compositions for the warm season averages, though the HAP data tends to plot slightly above the meteoric water line. The cool season HAP data is tightly clustered (with $\delta \mathrm{D}$ values between -105 and $-110 \%$ ) and does not overlap with the LLNL data ( $\delta \mathrm{D}$ values between -114 and $-122 \%$ ).

In general, the $\delta \mathrm{D}$ and $\delta^{18} \mathrm{O}$ values of meteoric waters become more depleted in heavy isotopes at lower air temperatures (Criss, 1999). Given that air temperature decreases with increasing altitude, precipitation falling at higher elevations is expected to have lower $\delta \mathrm{D}$ and $\delta^{18} \mathrm{O}$ values compared to precipitation that falls at lower elevations. The HAP sites are 500 to 1000 meters higher in elevation than the LLNL sites, and should therefore have lower $\delta$-values. However, the cool season $\delta \mathrm{D}$ and $\delta^{18} \mathrm{O}$ averages are actually higher at the HAP sites compared to the LLNL sites (Figure 5.3). This suggests a longer data record is needed to obtain reliable long-term averages for the HAP sites.

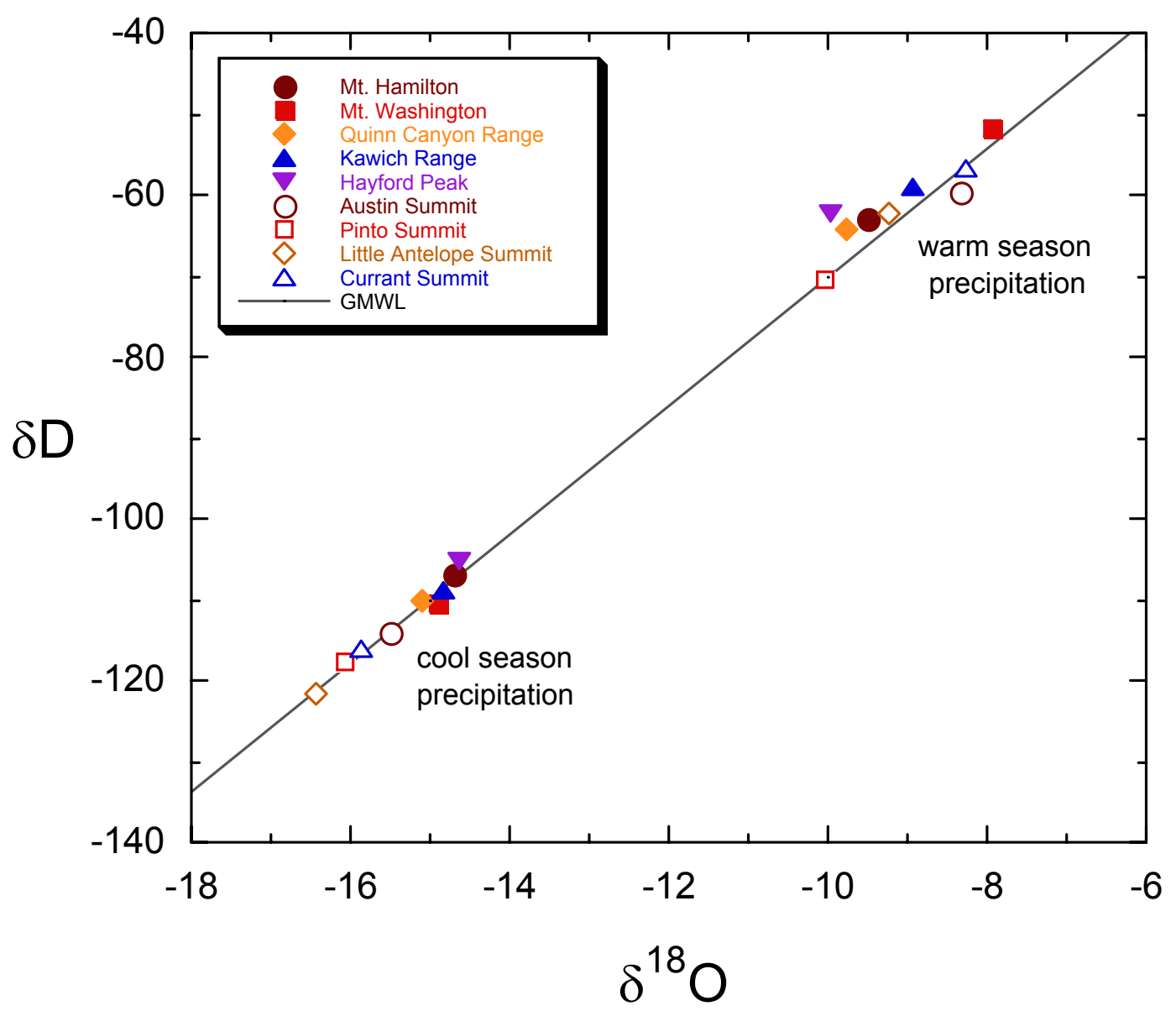

Figure 5.3 Plot of weighted mean $\delta D$ vs. $\delta^{18} \mathrm{O}$ values for cool- and warm-season precipitation samples collected from USGS HAP and LLNL central Nevada sites. GMWL $=$ global meteoric water line $\left(\delta D=8 \delta^{18} \mathrm{O}+10\right)$. 
Table 5.6 Precipitation Data for USGS HAP Sites

\begin{tabular}{|c|c|c|c|}
\hline $\begin{array}{l}\text { Location / } \\
\text { Sample Date }\end{array}$ & $\begin{array}{l}\text { Precipitation } \\
\text { Amount }(\mathrm{cm})\end{array}$ & $\begin{array}{l}\delta^{18} \mathrm{O} \\
(\% \circ)\end{array}$ & $\begin{array}{l}\delta \mathrm{D} \\
(\%)\end{array}$ \\
\hline \multicolumn{4}{|l|}{ Mt. Hamilton } \\
\hline 17-Oct-03 & 7.6 & -7.8 & -49 \\
\hline 16-Jun-04 & 29.8 & -15.4 & -116 \\
\hline 14-Oct-04 & 5.1 & -12.0 & -84 \\
\hline 06-Jul-05 & 111.0 & -14.5 & -105 \\
\hline 26-Oct-05 & n.d. & -9.5 & -66 \\
\hline 05-Jul-06 & 35.6 & -14.9 & -105 \\
\hline \multicolumn{4}{|c|}{ Mt. Washington } \\
\hline 16-Oct-03 & 5.7 & -7.9 & -52 \\
\hline 09-Jun-04 & 36.8 & -17.2 & -126 \\
\hline \multicolumn{4}{|c|}{ No sample collected in Oct. 2004 due to poor weather conditions } \\
\hline 12-Jul-05 & 116.8 & -14.7 & -110 \\
\hline \multicolumn{4}{|c|}{ No analysis in Oct. 2005 - sample damaged in shipment } \\
\hline 05-Jul-06 & 44.5 & -13.6 & -97 \\
\hline \multicolumn{4}{|c|}{ Quinn Canyon Range } \\
\hline 22-Oct-03 & 4.5 & -8.0 & -47 \\
\hline 16-Jun-04 & 17.1 & -15.6 & -121 \\
\hline 14-Oct-04 & 5.1 & -9.5 & -68 \\
\hline 06-Jul-05 & 48.9 & -15.9 & -115 \\
\hline 25-Oct-05 & 10.2 & -10.7 & -70 \\
\hline 23-Jun-06 & 21.6 & -13.0 & -91 \\
\hline \multicolumn{4}{|c|}{ Kawich Range } \\
\hline 22-Oct-03 & 10.2 & -7.4 & -41 \\
\hline 16-Jun-04 & 21.6 & -15.7 & -122 \\
\hline 14-Oct-04 & 3.2 & -9.9 & -69 \\
\hline 06-Jul-05 & 38.1 & -14.5 & -105 \\
\hline $26-O c t-05$ & 21.6 & -9.5 & -66 \\
\hline 23-Jun-06 & 21.0 & -14.7 & -104 \\
\hline \multicolumn{4}{|l|}{ Hayford Peak } \\
\hline 22-Oct-03 & 10.2 & -7.9 & -47 \\
\hline 16-Jun-04 & 25.4 & -15.5 & -115 \\
\hline 14-Oct-04 & 12.7 & -11.6 & -74 \\
\hline 06-Jul-05 & 70.5 & -14.4 & -102 \\
\hline 20-Oct-05 & 26.7 & -10.0 & -63 \\
\hline \multicolumn{4}{|c|}{ No sample collected in June 2006 - collection bag burst } \\
\hline
\end{tabular}

n.d. = no data (leaky drain plug on gauge) 
Table 5.7 Mean Precipitation Amounts and Isotopic Values for USGS HAP Sites

\begin{tabular}{lccccc}
\hline \hline & $\begin{array}{c}\text { Mount } \\
\text { Hamilton }\end{array}$ & $\begin{array}{c}\text { Mount } \\
\text { Washington }\end{array}$ & $\begin{array}{c}\text { Quinn Canyon } \\
\text { Range }\end{array}$ & $\begin{array}{c}\text { Kawich } \\
\text { Range }\end{array}$ & $\begin{array}{c}\text { Hayford } \\
\text { Peak }\end{array}$ \\
\hline \hline Full Record & & & & & \\
$\delta^{18} \mathrm{O}$ (wtd mean) & -14.4 & -14.7 & -14.1 & -13.1 & -13.1 \\
$\delta \mathrm{D}$ (wtd mean) & -104 & -109 & -102 & -94 & -91 \\
avg. amount (cm) & 37.8 & 51.0 & 17.9 & 19.3 & 29.1 \\
Warm Season & & & & & \\
$\delta^{18} \mathrm{O}$ (wtd mean) & -9.5 & -7.9 & -9.7 & -8.9 & -10.0 \\
$\delta \mathrm{D}$ (wtd mean) & -63 & -52 & -64 & -59 & -62 \\
avg. amount (cm) & 6.4 & 5.7 & 6.6 & 11.7 & 16.5 \\
Cool Season & & & & & \\
$\delta^{18} \mathrm{O}$ (wtd mean) & -14.7 & -14.9 & -15.1 & -14.8 & -14.6 \\
$\delta \mathrm{D}$ (wtd mean) & -107 & -110 & -110 & -109 & -105 \\
avg. amount (cm) & 58.8 & 66.0 & 29.2 & 26.9 & 47.95 \\
\hline
\end{tabular}

The precipitation gauges at Little Antelope Summit and Mt. Hamilton are located $19 \mathrm{~km}$ apart on the western side of the White Pine Range (Figure 5.1) at elevations of 2267 and $3230 \mathrm{~m}$, respectively (Table 5.1). Assuming these two sites experience similar weather patterns, we can predict the mean isotopic value of precipitation at Mt. Hamilton using data from Little Antelope Summit together with the empirical altitude-isotope relationship reported in the literature: $\Delta\left(\delta^{18} \mathrm{O}\right) / \Delta \mathrm{z}=(-2.0 \pm 1 \% \mathrm{c}) / \mathrm{km}$ (Criss, 1999). Precipitation at Little Antelope Summit had a mean annual $\delta^{18} \mathrm{O}$ value of $-14.9 \%$ for the period 1999-2006 (Table 5.3). Given the $963 \mathrm{~m}$ difference in elevation between Little Antelope Summit and Mt. Hamilton, we would predict a mean $\delta^{18} \mathrm{O}$ value of $-16.8 \pm$ $0.95 \%$ for Mt. Hamilton. The equivalent $\delta \mathrm{D}$ value is $-124 \%$. In comparison, the available three-year record for Mt. Hamilton yielded mean $\delta^{18} \mathrm{O}$ and $\delta \mathrm{D}$ values of -14.4 and $-104 \%$, respectively (Table 5.7). As the length of the record is extended over time, it will be interesting to see whether these data will 'evolve' to match the expected altitudeisotope effect.

Friedman et al. (2002) published a stable isotope study of precipitation in the Great Basin that includes data for Mt. Hamilton, Hayford Peak, and Austin (the latter is located only $\sim 2.5 \mathrm{~km}$ from Austin Summit). The Friedman et al. (2002) study was conducted from 1991 to 1997, and unfortunately none of the measurements overlap with the present study. Nevertheless, it is interesting to compare the two data records, which are coincidentally similar in duration. Data from Friedman et al. (2002) are shown side-byside with data from this study in Table 5.8. Comprehensive data were available only for $\delta \mathrm{D}$ in the Friedman et al. study. 
Table 5.8 Comparison of Cumulative $\delta \mathrm{D}$ and Average Precipitation Data from Friedman et al. (2002) and This Study

\begin{tabular}{|c|c|c|c|c|c|c|}
\hline & $\begin{array}{l}\text { Mount } \\
\text { Hamilton } \\
\text { (Friedman) }\end{array}$ & $\begin{array}{l}\text { Mount } \\
\text { Hamilton } \\
\text { (this study) }\end{array}$ & $\begin{array}{l}\text { Hayford } \\
\text { Peak } \\
\text { (Friedman) }\end{array}$ & $\begin{array}{c}\text { Hayford } \\
\text { Peak } \\
\text { (this study) }\end{array}$ & $\begin{array}{c}\text { Austin } \\
\text { (Friedman) }\end{array}$ & $\begin{array}{c}\text { Austin } \\
\text { Summit } \\
\text { (this study) }\end{array}$ \\
\hline $\begin{array}{l}\text { annual } \\
\quad \delta \mathrm{D} \text { (wtd. mean) }\end{array}$ & -105 & -104 & -95 & -91 & -120 & -105 \\
\hline $\begin{array}{l}\text { warm season } \\
\delta \mathrm{D} \text { (wtd. mean) } \\
\text { avg. amount }(\mathrm{cm}) \\
\text { number of collections }\end{array}$ & $\begin{array}{c}-90 \\
5.3 \\
5\end{array}$ & $\begin{array}{c}-63 \\
6.4 \\
2\end{array}$ & $\begin{array}{c}-89 \\
10.5 \\
3\end{array}$ & $\begin{array}{c}-62 \\
16.5 \\
3\end{array}$ & $\begin{array}{c}-109 \\
4.7 \\
5\end{array}$ & $\begin{array}{c}-60 \\
4.3 \\
5\end{array}$ \\
\hline $\begin{array}{l}\text { cool season } \\
\delta \mathrm{D} \text { (wtd. mean) } \\
\text { avg. amount }(\mathrm{cm}) \\
\text { number of collections }\end{array}$ & $\begin{array}{c}-111 \\
36.2 \\
2\end{array}$ & $\begin{array}{c}-107 \\
58.8 \\
3\end{array}$ & $\begin{array}{c}-103 \\
30.8 \\
3\end{array}$ & $\begin{array}{c}-105 \\
48.0 \\
2\end{array}$ & $\begin{array}{c}-125 \\
13.1 \\
4\end{array}$ & $\begin{array}{c}-114 \\
14.2 \\
5\end{array}$ \\
\hline
\end{tabular}

At Mt. Hamilton and Hayford Peak, the annual and cool season mean $\delta \mathrm{D}$ values do not show major differences between the two datasets, but the warm season $\delta \mathrm{D}$ values differ by $27 \%$ at both locations (Table 5.8). The difference in warm season data is quite striking and may be indicative of warmer summer air temperatures during storm events in 2003 to 2005 as compared to the early- to mid-1990s.

Comparison of the Austin and Austin Summit records reveals that mean $\delta \mathrm{D}$ values from Friedman et al. (2002) are lower than those from this study in all categories, with the greatest difference occurring in the warm season data (49\% different). Moreover, the elevation of the Austin collection station used in Friedman's study was $1747 \mathrm{~m}$ whereas that of the Austin Summit gauge is $2285 \mathrm{~m}$ - a difference of $538 \mathrm{~m}$. We would therefore expect the precipitation from the Austin site to have $\delta \mathrm{D}$ values that are higher (less negative) than the Austin Summit data. This is not consistent with the data comparison in Table 5.8. Further investigation into the source of these differences is warranted.

\subsection{Summary and Conclusions}

Stable isotope monitoring of precipitation, spring discharge, and soil water has been conducted at four sites in central Nevada since 1999. In addition, precipitation samples from five sites in eastern Nevada have been analyzed for stable isotopes since 2003 . The latter sites are part of the USGS High Altitude Precipitation (HAP) monitoring network. The data from these sites can be used to improve our understanding of precipitation and recharge processes in Nevada, and can be applied to the development of conceptual models of hydrologic processes, for validating numerical groundwater flow models, and for monitoring potential changes in climate over time. 
Analysis of the existing data led to the following conclusions, most of which are based on the dataset developed for the four LLNL sites in central Nevada.

- Warm season precipitation (May through October) accounts for only 20 to $23 \%$ of the annual precipitation total. The majority of the annual precipitation (77 to $80 \%$ ) occurs during the cool season, primarily as winter snowfall.

- Precipitation samples have stable isotope compositions that plot very near the global meteoric water line. Cumulative annual mean $\delta \mathrm{D}$ and $\delta^{18} \mathrm{O}$ values for precipitation are skewed toward the cool season average, reflecting the fact that most precipitation occurs during the winter season.

- Weighted mean $\delta \mathrm{D}$ and $\delta^{18} \mathrm{O}$ values for soil waters (lysimeters) plot close to the meteoric water line at three out of four locations, and are depleted in heavy isotopes relative to the average cool season precipitation. This suggests that snowmelt-derived recharge occurs primarily during the early stages of melting, when the snowmelt exhibits the greatest depletion in heavy isotopes.

- The average $\delta \mathrm{D}$ and $\delta^{18} \mathrm{O}$ values of perched springs can be derived from the weighted mean $\delta \mathrm{D}$ and $\delta^{18} \mathrm{O}$ values of soil waters at all four central Nevada sites. At three of the four sites, the perched springs appear to be related to the soil water through evaporation processes. At one location, the spring and soil water have nearly identical compositions that lie on the meteoric water line.

- Soil moisture evaporation is considered to be the most plausible mechanism to explain the evaporated isotope signatures of the perched springs.

- Weighted mean isotopic values for precipitation samples from the USGS HAP sites have cool and warm season averages similar to that of the four central Nevada sites. However, the cool season HAP data has mean $\delta \mathrm{D}$ and $\delta^{18} \mathrm{O}$ values that are greater than the central Nevada data despite the fact that the former is collected at higher altitudes. This suggests a longer data record is needed to obtain reliable long-term averages.

- Comparison of precipitation data from this study with data published by Friedman et al. (2002) for several of the same locations shows some discrepancies in the cumulative average $\delta \mathrm{D}$ values, particularly for the warm season samples. The source of these differences warrants further investigation. 


\subsection{References}

Allison, G.B., Barnes, C.J., and Hughes, M.W. (1983) The distribution of deuterium and

${ }^{18} \mathrm{O}$ in dry soils. 2. Experimental. Jour. Hydrology, 64: 377-397.

Arnason, B., Buason, T., Martinec, J., and Theodorsson, P. (1973) Movement of water through snow pack traced by deuterium and tritium. In: The Role of Snow and Ice in Hydrology. Proceedings of the Banff Symposia, September 1972, vol. 1, UNESCOWMO-IAHS Publication No. 107, p. 299-312.

Craig, H. (1961) Isotopic variations in meteoric waters. Science, 133: 1702-1703.

Criss, R.E. (1999) Principles of Stable Isotope Distribution. Oxford, New York, 254 p.

Fontes, J.Ch. (1980) Environmental Isotopes in Groundwater Hydrology. In: P.Fritz and J.Ch. Fontes, eds., Handbook of Environmental Isotope Geochemistry, vol. 1, The Terrestrial Environment, A, Elsevier, Amsterdam, p. 75-140.

Friedman, I., Smith, G.I., Johnson, C.A., and Moscati, R.J. (2002) Stable isotope compositions of waters in the Great Basin, United States 2. Modern precipitation. Journal of Geophysical Research, vol. 107, no. D19, 4401, doi:10.1029/2001JD000566.

Herrmann, A., Lehrer, M., and Stichler, W. (1981) Isotope input to runoff systems from melting snow covers. Nordic Hydrology: 12, 309-318.

O'Neil, J.R. (1968) Hydrogen and oxygen isotope fractionation between ice and water. Jour. Phys. Chem. 72: 3683-3684.

Prudic, D.E., Harrill, J.R., and Burbey, T.J. (1995) Conceptual Evaluation of Regional Ground-Water Flow in the Carbonate-Rock Province of the Great Basin, Nevada, Utah, and Adjacent States. U.S. Geol. Survey Professional Paper 1409-D, 102 p.

Rose, T.P., Davisson, M.L., Criss, R.E., and Smith, D.K. (1999) Isotopic investigation of recharge to a regional groundwater flow system, Great Basin, Nevada, USA. In: Proceedings International Symposium on Isotope Techniques in Water Resources Development and Management, Vienna, 10-14 May 1999. International Atomic Energy Agency, IAEA-CSP-2/C, session 2, p. 63-72.

Rose, T.P., Eaton, G.F., and Kersting, A.B., eds. (2003) Hydrologic Resources Management Program and Underground Test Area Project FY2001-2002 Progress Report. Lawrence Livermore National Laboratory Report UCRL-ID-154357, August 2003, 134 p.

Rose, T.P., Eaton, G.F., and Kersting, A.B., eds. (2004) Hydrologic Resources Management Program and Underground Test Area Project FY2003 Progress Report. Lawrence Livermore National Laboratory Report UCRL-ID-206661, August 2004, 81 p. 
Singleton, M.J., Sonnenthal, E.L., Conrad, M.E., DePaolo, D.J., and Gee, G.W. (2004) Multiphase reactive transport modeling of seasonal infiltration events and stable isotope fractionation in unsaturated zone pore water and vapor at the Hanford Site. Vadose Zone Journal, 3: 775-785.

Stichler, W., Rauert, W., and Martinec, J. (1981) Environmental isotope studies of an alpine snowpack. Nordic Hydrology: 12, 297-308.

Thomas, J.M., Welch, A.H., and Dettinger, M.D. (1996) Geochemistry and Isotope Hydrology of Representatitve Aquifers in the Great Basin Region of Nevada, Utah, and Adjacent States. U.S. Geol. Survey Professional Paper 1409-C, 100 p.

U.S. Geological Survey (2005) BARCASS Factsheet. U.S. Geol. Survey Fact Sheet 2005-3035.

U.S. Geological Survey (2006) Water Resources Data - Nevada, Water Year 2005. U.S. Geol. Survey Water-Data Report NV-05-1. 


\section{Distribution}

\section{Organizations:}

U.S. Department of Energy

National Nuclear Security Administration

Nevada Site Office

Technical Information Resource Center

P.O. Box 98518

Las Vegas, NV 89193

U.S. Department of Energy

National Nuclear Security Administration

Nevada Site Office

Public Reading Facility

P.O. Box 98521

Las Vegas, NV 89193

U.S. Department of Energy

Office of Scientific and Technical Information

P.O. Box 62

Oak Ridge, TN 37831-0062

\section{Individuals:}

Stacey Alderson

MS 439

7710 W. Cheyenne Ave.

Las Vegas, NV 89129

Chris Andres

Nevada Department of Conservation

\& Natural Resources

Division of Environmental Protection

2030 E. Flamingo Rd, Suite 230

Las Vegas, NV 89119-0818

Naomi Becker

P.O. Box 1663, MS T003

Los Alamos NM 87545

Pat Bodin

NNSA/NSO

P.O. Box 98518

Las Vegas, Nevada 89193

Carol Bruton

Lawrence Livermore National Lab

P.O. Box 808, L-221

Livermore, California 94551-9900

Steven F. Carle

Lawrence Livermore National Lab

P.O. Box 808, L-221

Livermore, California 94551-9900

Bruce Crowe

NNSA/NSO

MS 505

P.O. Box 98518

Las Vegas, Nevada 89193

Jeff Daniels

Lawrence Livermore National Lab

P.O. Box 808, L-072

Livermore, California 94551-9900

Barb Deshler

Stoller-Navarro Joint Venture

7710 W. Cheyenne Ave.

Las Vegas, Nevada 89129

Dee Donithan

Desert Research Institute

755 E. Flamingo

Las Vegas, Nevada 89119 
Dave Donovan

SNWA Resources

1900 E. Flamingo

Suite 253

Las Vegas, NV 89119

Sig Drellack

NSTec

P.O. Box $98521 \mathrm{MS} / \mathrm{NLV} 082$

Las Vegas, Nevada 89193

Doug Duncan

U.S. Geological Survey

411 National Center

Reston, VA 20192

Brad Esser

Lawrence Livermore National Laboratory

P.O. Box 808, L-231

Livermore, California 94551-9900

Irene Farnham

Stoller-Navarro Joint Venture

7710 W. Cheyenne Ave.

Las Vegas, Nevada 89129

Dave Finnegan

Los Alamos National Laboratory

P.O. Box 1663, MS-J514 C-NR

Los Alamos, New Mexico 87545

Robert Graves

U.S. Geological Survey

160 N. Stephanie Street

Henderson, NV 89074-8829

Ward Hawkins

Los Alamos National Laboratory

P.O. Box 1663, MS-F665 / EES-11

Los Alamos, New Mexico 87545

Ron Hershey

Desert Research Institute

2215 Raggio Parkway

Reno, Nevada 89512

Max $\mathrm{Hu}$

Lawrence Livermore National Laboratory

P.O. Box 808, L-231

Livermore, California 94551-9900

Bruce Hurley

NNSA/NSO

P.O. Box 98518

Las Vegas, Nevada 89193
Britt Jacobson

Nevada Department of Conservation

\& Natural Resources

Division of Environmental Protection

2030 E. Flamingo Rd, Suite 230

Las Vegas, NV 89119-0818

Roger Jacobson

Desert Research Institute

2215 Raggio Parkway

Reno, Nevada 89512

Jackie Kenneally

Lawrence Livermore National Laboratory

P.O. Box 808, L-236

Livermore, California 94551-9900

Annie Kersting

Lawrence Livermore National Laboratory

P.O. Box 808, L-231

Livermore, California 94551-9900

Ed Kwicklis

Los Alamos National Laboratory

Box 1663, T003

Los Alamos, New Mexico 87545

Randy Laczniak

U.S. Geological Survey

160 N. Stephanie Street

Henderson, NV 89074-8829

Stephen Leedom

NNSA/NSO

P.O. Box 98518

Las Vegas, Nevada 89193

Joseph Leising

SNWA Resources

1900 E. Flamingo

Suite 253

Las Vegas, NV 89119

Rachel Lindvall

Lawrence Livermore National Laboratory

P.O. Box 808, L-231

Livermore, California 94551-9900

Reed Maxwell

Lawrence Livermore National Laboratory

P.O. Box 808, L-208

Livermore, California 94551-9900 
John McCord

Stoller-Navarro Joint Venture

7710 W. Cheyenne Ave.

Las Vegas, NV 89129

Mark McLane

Nevada Department of Conservation

\& Natural Resources

Division of Environmental Protection

2030 E. Flamingo Rd, Suite 230

Las Vegas, NV 89119-0818

Jean Moran

Lawrence Livermore National Laboratory

P.O. Box 808, L-231

Livermore, California 94551-9900

Ken Ortego

NSTec

P.O. Box $98521 \mathrm{M} / \mathrm{S}$ NSF 082

Las Vegas, Nevada 89193

Jim Paces

U.S. Geological Survey

P.O. Box 25046, MS 963

Denver Federal Center

Denver, CO 80225

Gayle Pawloski

Lawrence Livermore National Laboratory

P.O. Box 808, L-221

Livermore, California 94551-9900

Zell Peterman

U.S. Geological Survey

P.O. Box 25046 M/S 963

Denver Federal Center

Denver, Colorado 80225

Greg Pohll

Desert Research Institute

2215 Raggio Parkway

Reno, Nevada 89512

David Prudic

US Geological Survey

333 West Nye Lane Rm 203

Carson City, Nevada 89706

Paul Reimus

Los Alamos National Laboratory

P.O. Box 1663, MS-J534 CST-7

Los Alamos, New Mexico 87545
Timothy Rose

Lawrence Livermore National Laboratory

P.O. Box 808, L-236

Livermore, California 94551-9900

Greg Ruskauff

Stoller-Navarro Joint Venture

7710 W. Cheyenne Ave.

Las Vegas, Nevada 89129

Chuck Russell

Desert Research Institute

755 E. Flamingo

Las Vegas, Nevada 89119

David K. Smith

Lawrence Livermore National Laboratory

P.O. Box 808, L-171

Livermore, California 94551-9900

Jim Thomas

Desert Research Institute

2215 Raggio Parkway

Reno, Nevada 89512

Bonnie Thompson

U.S. Geological Survey

160 N. Stephanie Street

Henderson, NV 89074-8829

K.C. Thompson

Environmental Restoration Division

NNSA/NSO

P.O. Box 98521 MS 505

232 Energy Way

Las Vegas, Nevada 89193

Andy Tompson

Lawrence Livermore National Laboratory

P.O. Box 808, L-208

Livermore, California 94551-9900

Rick Waddell

GeoTrans Inc.

363 Centennial Parkway

Suite 210

Louisville, CO 80027

Bill Wilborn

Environmental Restoration Division

NNSA/NSO

P.O. Box 98515 MS 505

232 Energy Way

Las Vegas, Nevada 89193 
Ross Williams

Lawrence Livermore National Laboratory

P.O. Box 808, L-231

Livermore, California 94551-9900

Jon Wilson

U.S. Geological Survey

160 N. Stephanie Street

Henderson, NV 89074

Andy Wolfsberg

Los Alamos National Laboratory

Box 1663, MS T003

Los Alamos, New Mexico 87545

Jeff Wurtz

Stoller-Navarro Joint Venture

MS 439

7710 W. Cheyenne Ave.

Las Vegas, Nevada 89129

Vefa Yucel

Bechtel Nevada

P.O. Box $98521 \mathrm{MS} / \mathrm{NLV} 81$

Las Vegas, Nevada 89193

Mavrik Zavarin

Lawrence Livermore National Laboratory

P.O. Box 808, L-231

Livermore, California 94551-9900

Pihong Zhao

Lawrence Livermore National Laboratory

P.O. Box 808, L-231

Livermore, California 94551-9900 


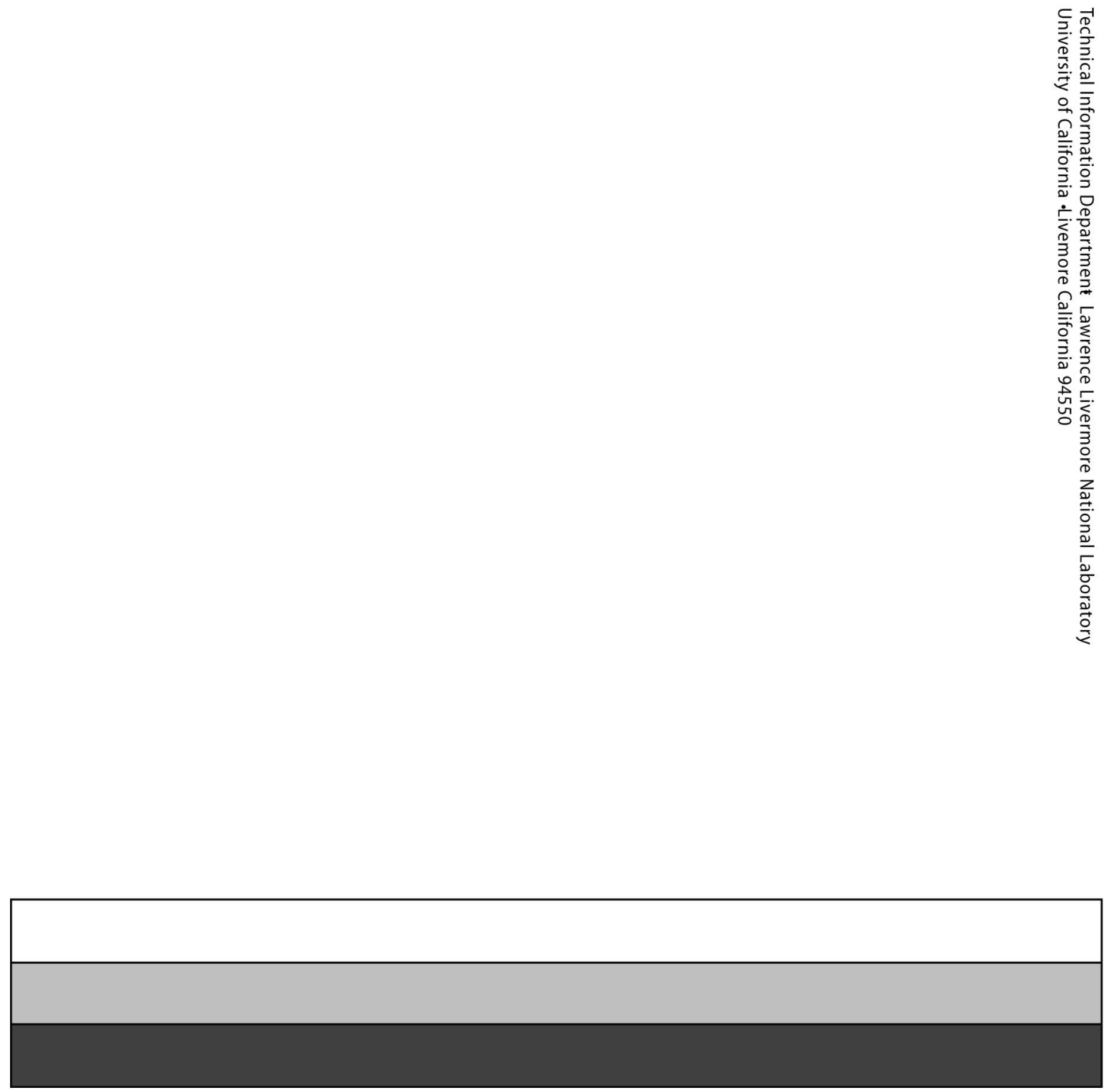